\title{
Baseline Risk Assessment for the Groundwater Operable Units at the Chemical Plant Area and the Ordnance Works Area, Weldon Spring, Missouri
}

July 1997

prepared by

Environmental Assessment Division, Argonne National Laboratory

\section{prepared for}

U.S. Department of Energy, Weldon Spring Site Remedial Action Project, Weldon Spring, Missouri, under Contract W-31-109-Eng-38 and U.S. Department of the Army, Corps of Engineers,

Kansas City District, Kansas City, Missouri 
6ry

This report is printed on recycled paper. 


\section{DISCLAIMER}

This report was prepared as an account of work sponsored by an agency of the United States Government. Neither the United States Government nor any agency thereof, nor any of their employees, make any warranty, express or implied, or assumes any legal liability or responsibility for the accuracy, completeness, or usefulness of any information, apparatus, product, or process disclosed, or represents that its use would not infringe privately owned rights. Reference herein to any specific commercial product, process, or service by trade name, trademark, manufacturer, or otherwise does not necessarily constitute or imply its endorsement, recommendation, or favoring by the United States Government or any agency thereof. The views and opinions of authors expressed herein do not necessarily state or reflect those of the United States Government or any agency thereof. 


\section{DISCLAIMER}

Portions of this document may be illegible in electronic image products. Images are produced from the best available original document. 


\section{CONTENTS}

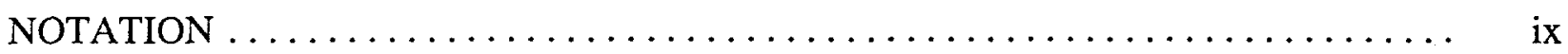

ENGLISH/METRIC AND METRIC/ENGLISH EQUIVALENTS $\ldots \ldots \ldots \ldots \ldots$ xi

1 INTRODUCTION $\ldots \ldots \ldots \ldots \ldots \ldots \ldots \ldots \ldots \ldots \ldots \ldots \ldots \ldots \ldots \ldots \ldots \ldots \ldots$

1.1 Scope of the Baseline Risk Assessment $\ldots \ldots \ldots \ldots \ldots \ldots \ldots \ldots \ldots \ldots \ldots \ldots$

1.2 Purpose of this Baseline Risk Assessment $\ldots \ldots \ldots \ldots \ldots \ldots \ldots \ldots \ldots \ldots \ldots . \ldots$

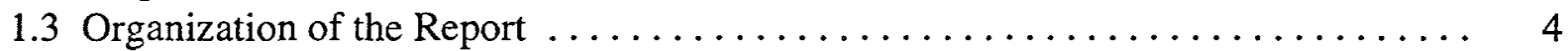

2 IDENTIFICATION OF CONTAMINANTS OF CONCERN $\ldots \ldots \ldots \ldots \ldots \ldots$

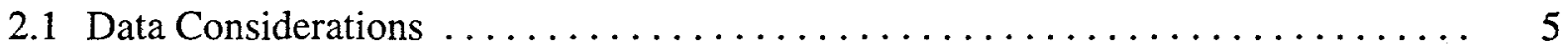

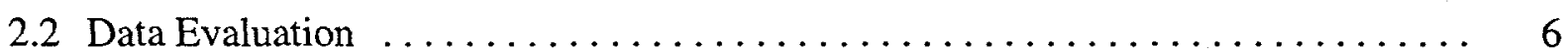

3 EXPOSURE AND EFFECTS ASSESSMENT $\ldots \ldots \ldots \ldots \ldots \ldots \ldots \ldots \ldots$

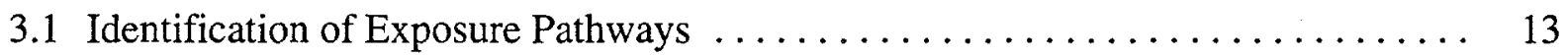

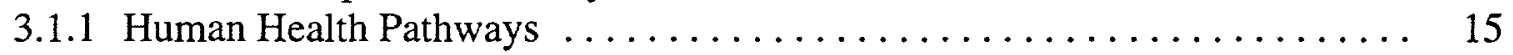

3.1 .2 Ecological Effects . . . . . . . . . . . . . . . . . . . . 18

3.2 Estimation of Exposure Point Concentrations $\ldots \ldots \ldots \ldots \ldots \ldots \ldots \ldots \ldots$

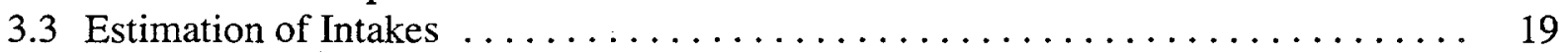

3.3.1 Radiological Intakes . . . . . . . . . . . . . . . . . . . . . 20

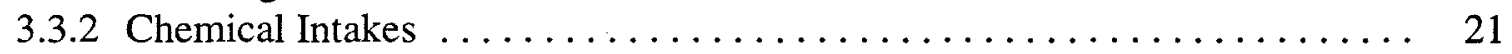

3.4 Ecological Resources Exposure and Effects Assessment . . . . . . . . . . 22

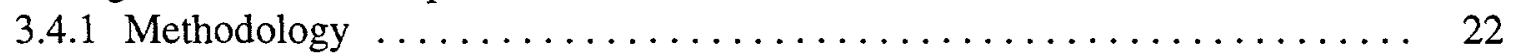

3.4 .2 Toxicity Results ............................. 24

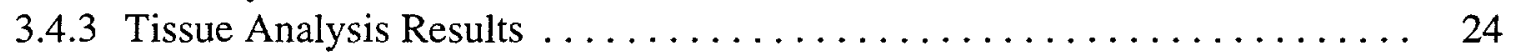

3.4 .4 Biotic Survey Results . . . . . . . . .

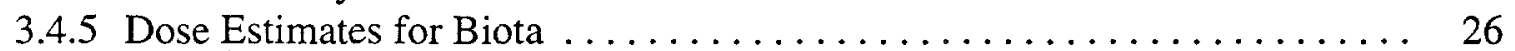

4 TOXICITY ASSESSMENT $\ldots \ldots \ldots \ldots \ldots \ldots \ldots \ldots \ldots \ldots \ldots \ldots \ldots \ldots \ldots$

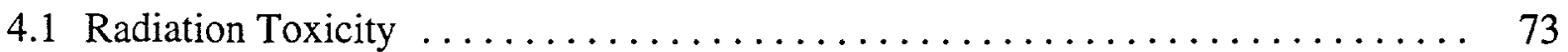

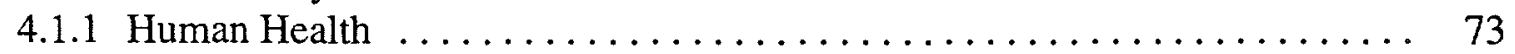

4.1 .2 Ecological Health . . . . . . . . . . . . . . . . . . . . 73

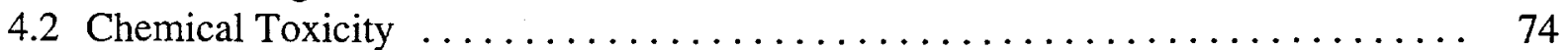

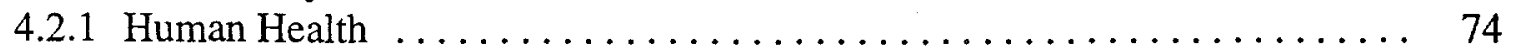

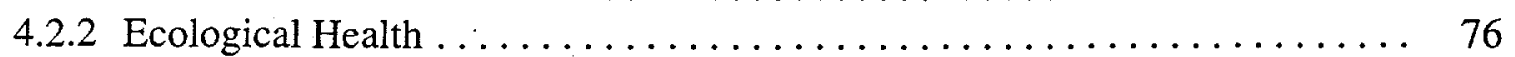

4.3 Methods for Evaluating Toxicity to Human Health $\ldots \ldots \ldots \ldots \ldots \ldots \ldots$

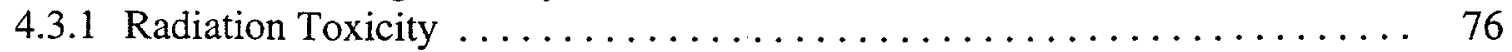

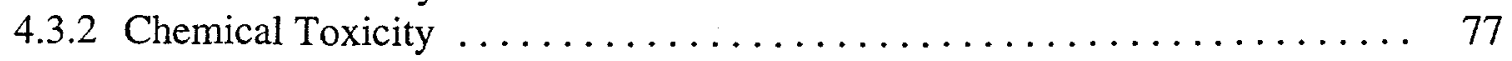




\section{CONTENTS (Cont.)}

5 HUMAN HEALTH RISK CHARACTERIZATION $\ldots \ldots \ldots \ldots \ldots \ldots \ldots \ldots \ldots$

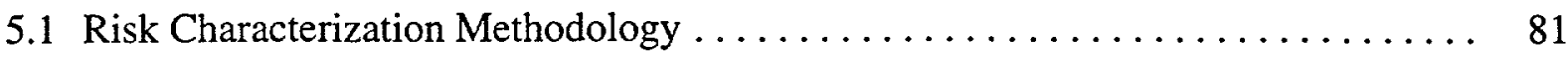

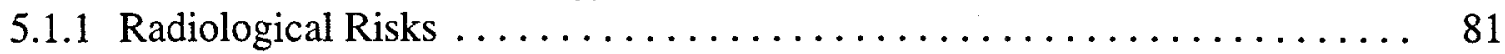

5.1.2 Chemical Risks and Hazard Quotients $\ldots \ldots \ldots \ldots \ldots \ldots \ldots \ldots \ldots . \quad 82$

5.1.2.1 Carcinogenic Risks ......................... 82

5.1.2.2 Hazard Quotients and Hazard Indices $\ldots \ldots \ldots \ldots \ldots \ldots \ldots . \quad 82$

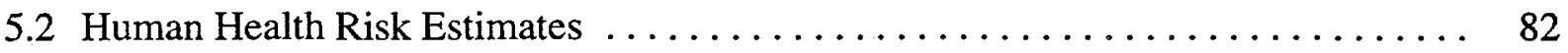

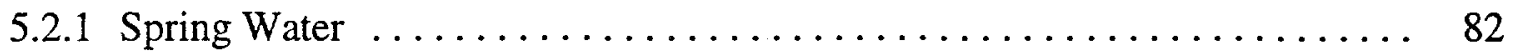

5.2.1.1 Chemical Risks and Hazard Indices $\ldots \ldots \ldots \ldots \ldots \ldots \ldots \ldots . \ldots 3$

5.2.1.2 Radiological Risks $\ldots \ldots \ldots \ldots \ldots \ldots \ldots \ldots \ldots \ldots \ldots \ldots$

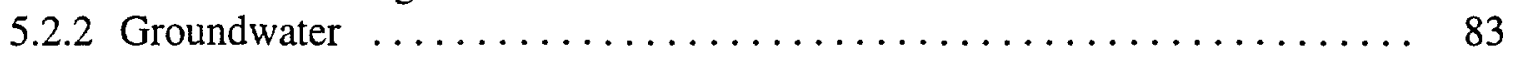

5.2.2.1 Chemical Risks and Hazard Indices . . . . . . . . . . . . 85

5.2.2.2 Radiological Risks ......................... 87

5.3 Uncertainties Related to Risk Estimates $\ldots \ldots \ldots \ldots \ldots \ldots \ldots \ldots \ldots . \ldots \ldots$

5.3.1 Contaminants of Potential Concern $\ldots \ldots \ldots \ldots \ldots \ldots \ldots \ldots \ldots . \quad 87$

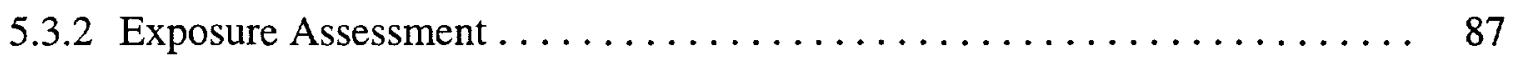

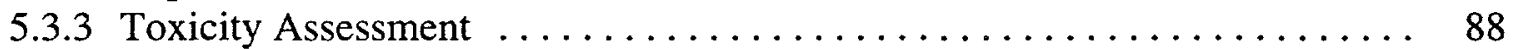

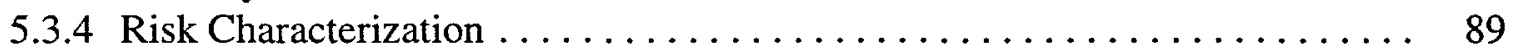

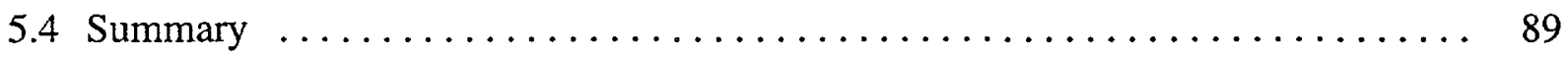

5.5 Cumulative Exposure through Multiple Media $\ldots \ldots \ldots \ldots \ldots \ldots \ldots \ldots .90$

6 ECOLOGICAL RISK CHARACTERIZATION $\ldots \ldots \ldots \ldots \ldots \ldots \ldots \ldots \ldots \ldots$

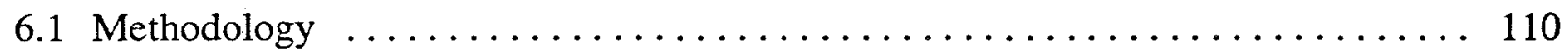

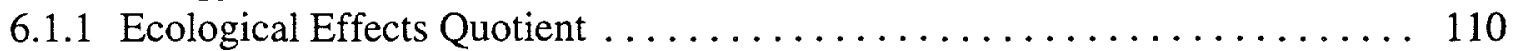

6.1.1.1 Calculation ............................... 110

6.1.1.2 Benchmark Values $\ldots \ldots \ldots \ldots \ldots \ldots \ldots \ldots \ldots \ldots \ldots \ldots \ldots$

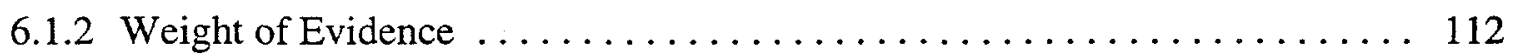

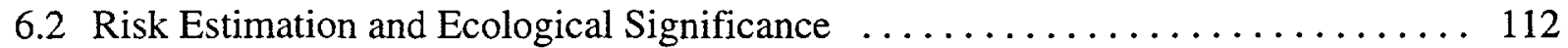

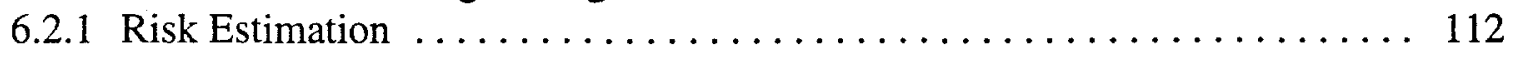

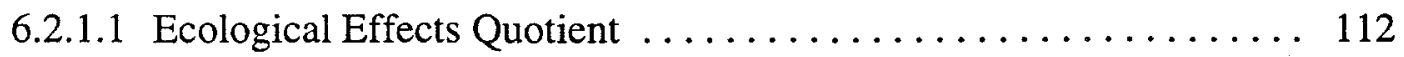

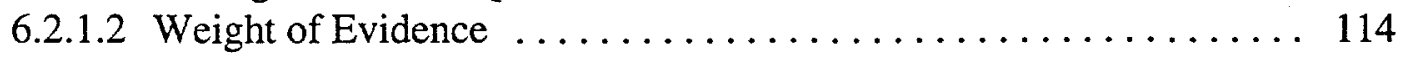

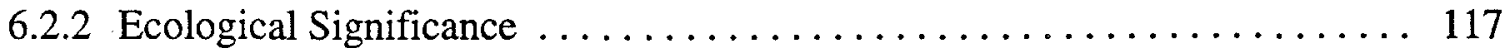

6.3 Uncertainties Related to Risk Characterization $\ldots \ldots \ldots \ldots \ldots \ldots \ldots \ldots \ldots$

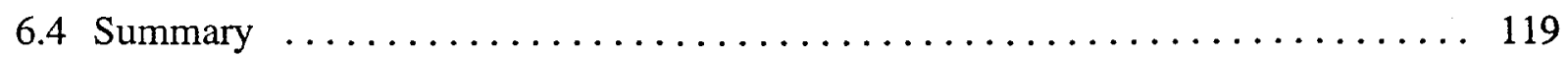

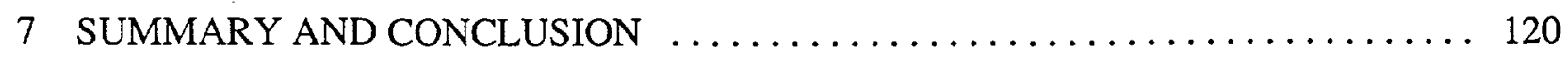

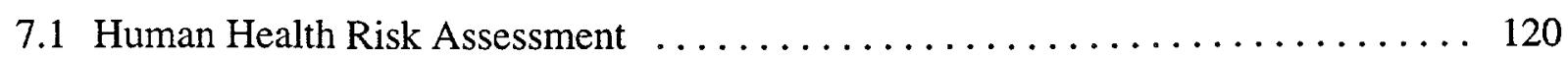

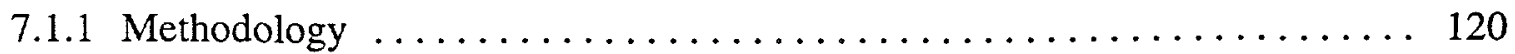

7.1 .2 Results . . . . . . . . . . . . . . . . . . . . . . . . . . . . 121

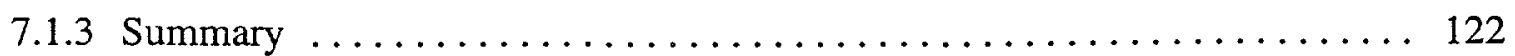




\section{CONTENTS (Cont.)}

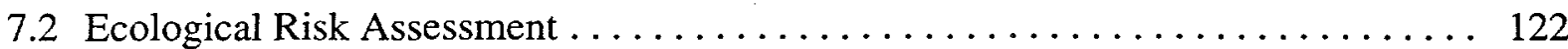

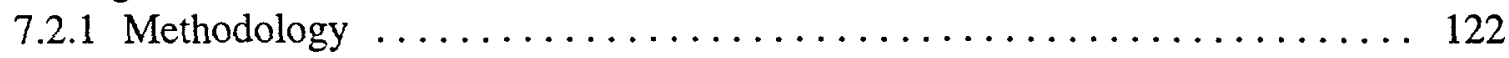

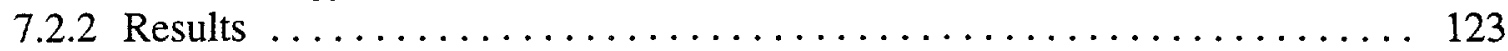

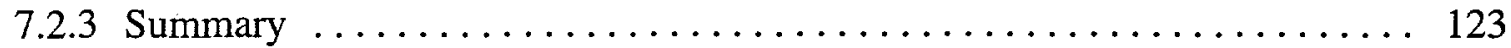

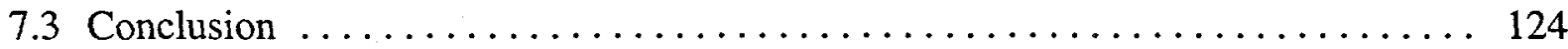

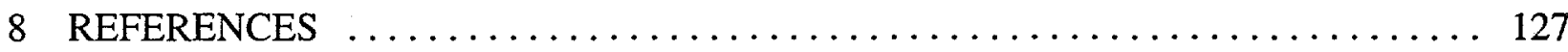

\section{FIGURES}

1.1 Locations of the Chemical Plant Area and the Ordnance Works Area $\ldots \ldots \ldots \ldots .2$

2.1 Locations of Monitoring Wells and Springs at the Chemical Plant Area

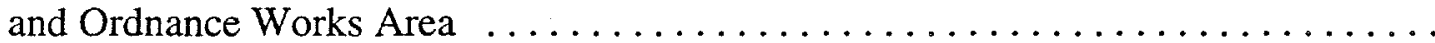

3.1 Map of the Chemical Plant Area and the Ordnance Works Area and Immediate Vicinity

3.2 Conceptual Site Exposure Model for the Chemical Plant Area and Ordnance Works Area

5.1 Distribution of Estimated Hazard Indices for the Current and Future Recreational Visitor at the Chemical Plant Area and Ordnance

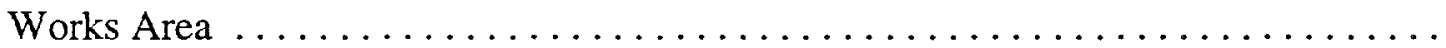

5.2 Distribution of Estimated Chemical and Radiological Carcinogenic Risks for the Current and Future Recreational Visitor at the Chemical Plant Area and Ordnance Works Area

5.3 Distribution of Estimated Hazard Indices for the Hypothetical Future Resident at the Chemical Plant Area and Ordnance Works Area ..............

5.4 Distribution of Estimated Chemical and Radiological Carcinogenic Risks for the Hypothetical Future Resident at the Chemical Plant

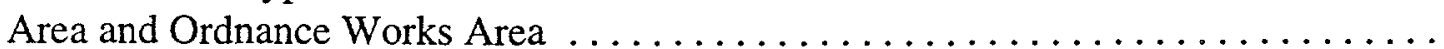

7.1 Monitoring Wells and Springs with the Highest Estimated Risks and Hazard Indices 


\section{TABLES}

2.1 Groundwater and Spring Water Contaminants $\ldots \ldots \ldots \ldots \ldots \ldots \ldots \ldots$

2.2 Screening of Sediment from the Burgermeister Spring System

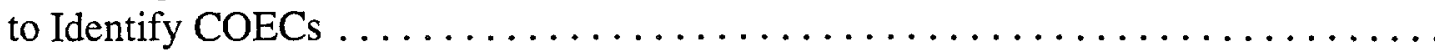

2.3 Screening of Surface Water from Springs in the Chemical Plant Area and Ordnance Works Area to Identify COECs .................. 11

3.1 Exposure Scenario Assumptions and Intake Parameters ................ 27

3.2 Estimated Noncarcinogenic Intakes of COPCs for the Current

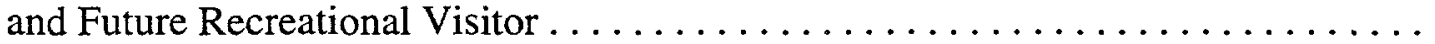

3.3 Estimated Carcinogenic Intakes of COPCs for the Current

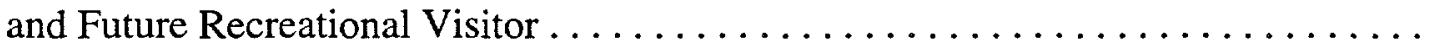

3.4 Estimated Intakes of Uranium for the Current and Future Recreational Visitor . . . . . . . . . . . . . . . . . . . . . . . . . .

3.5 Estimated Noncarcinogenic Intakes of Metal COPCs for the Hypothetical Future Resident

3.6 Estimated Noncarcinogenic Intakes of Inorganic Anion COPCs

for the Hypothetical Future Resident

3.7 Estimated Noncarcinogenic Intakes of Organic Compound COPCs

for the Hypothetical Future Resident

3.8 Estimated Carcinogenic Intakes of Organic Compound COPCs for the Hypothetical Future Resident

3.9 Estimated Intakes of Uranium for the Hypothetical Future Resident $\ldots \ldots \ldots \ldots$

3.10 Exposure Factors for the American Robin

3.11 Exposure Factors for the White-Tailed Deer

3.12 Results of Acute and Chronic Toxicity Testing of Surface Water from Burgermeister Spring

3.13 Results of Acute and Chronic Toxicity Testing of Sediment from Burgermeister Spring 


\section{TABLES (Cont.)}

3.14 Estimated Applied Daily Dose from the Drinking Water Pathway for the American Robin and White-Tailed Deer .......................

4.1 Toxicity Values of COPCs for Ingestion of Groundwater and Spring Water: Potential Systemic Effects . . . . . . . . . . . . . . . . . . 79

4.2 Toxicity Values of COPCs for Ingestion of Groundwater and Spring Water: Potential Carcinogenic Effects . . . . . . . . . . . . . . . . 80

5.1 Estimated Hazard Quotients for COPCs for the Current

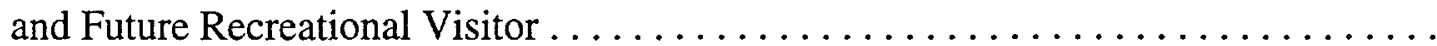

5.2 Estimated Chemical Carcinogenic Risks for the Current and Future Recreational Visitor

5.3 Estimated Radiological Carcinogenic Risks for the Current and Future Recreational Visitor.

5.4 Estimated Hazard Quotients for the Hypothetical Future Resident

5.5 Estimated Chemical Carcinogenic Risks to the Hypothetical

Future Resident for the Ingestion Pathway

5.6 Estimated Radiological Carcinogenic Risks for the Hypothetical

Future Resident

6.1. Benchmark Values Used to Estimate EEQs for Aquatic and Terrestrial Biota

6.2 Estimated EEQs for Aquatic Biota Exposed to Surface Water and Sediment at Burgermeister Spring

6.3 Estimated EEQs for Terrestrial Biota Drinking Water from Springs

6.4 Summary of the Ecological Effects Assessment of Burgermeister Spring 


\section{NOTATION}

The following is a list of the acronyms, initialisms, and abbreviations (including units of measure) used in this document. Some acronyms used in tables or equations only are defined in the respective tables or equations.

\section{ACRONYMS, INITIALISMS, AND ABBREVIATIONS}

\section{General}

$\begin{array}{ll}\text { ADD } & \text { applied daily dose } \\ \text { AWQC } & \text { ambient water quality criteria } \\ \text { BCF } & \text { bioconcentration factor } \\ \text { BRA } & \text { baseline risk assessment (this document) } \\ \text { CERCLA } & \text { Comprehensive Environmental Response, Compensation, and Liability } \\ & \text { Act of 1980, as amended } \\ \text { COEC } & \text { contaminant of ecological concern } \\ \text { COPC } & \text { contaminant of potential concern } \\ \text { DA } & \text { U.S. Department of the Army } \\ \text { DOE } & \text { U.S. Department of Energy } \\ \text { EEQ } & \text { ecological effects quotient } \\ \text { EPA } & \text { U.S. Environmental Protection Agency } \\ \text { EPC } & \text { exposure point concentration } \\ \text { FS } & \text { feasibility study } \\ \text { GWOU } & \text { groundwater operable unit } \\ \text { IAEA } & \text { International Atomic Energy Agency } \\ \text { IRIS } & \text { Integrated Risk Information System (EPA) } \\ \text { IT } & \text { International Technology (Corporation) } \\ \text { LOAEL } & \text { lowest-observed-adverse-effect level } \\ \text { MCL } & \text { maximum contaminant level } \\ \text { NCRP } & \text { National Commission on Radiation Protection and Measurements } \\ \text { NOAEL } & \text { no-observed-adverse-effect level } \\ \text { NPL } & \text { National Priorities List } \\ \text { RDA } & \text { recommended daily allowance } \\ \text { RfD } & \text { reference dose } \\ \text { RI } & \text { remedial investigation } \\ \text { UCL } & 95 \% \text { upper confidence limit of the arithmetic average } \\ \text { USGS } & \text { U.S. Geological Survey } \\ & \end{array}$




\section{Compounds}

1,2-DCE

1,3-DNB

DNT

2-amino-4,6-DNT

4-amino-2,6-DNT

2,4-DNT

2,6-DNT

TCE

$1,3,5-\mathrm{TNB}$

TNT

2,4,6-TNT 1,2-dichloroethylene

1,3-dinitrobenzene

dinitrotoluene

2-amino-4,6-dinitrotoluene

4-amino-2,6-dinitrotoluene

2,4-dinitrotoluene

2,6-dinitrotoluene

trichloroethylene

1,3,5-trinitrobenzene

trinitrotoluene

2,4,6-trinitrotoluene

\section{UNITS OF MEASURE}

$\mathrm{Ci} \quad$ curie(s)

$\mathrm{cm}$ centimeter(s)

$\mathrm{cm}^{2} \quad$ square centimeter(s)

$\mathrm{cm}^{3} \quad$ cubic centimeter(s)

d day(s)

$\mathrm{ft}$ foot (feet)

g $\operatorname{gram}(\mathrm{s})$

h hour(s)

ha hectare(s)

kg kilogram(s)

$\mathrm{km} \quad$ kilometer(s)
L liter(s)

$\mu \mathrm{g} \quad \operatorname{microgram}(\mathrm{s})$

$\mathrm{m}$ meter(s)

$\mathrm{m}^{3} \quad$ cubic meter(s)

mg milligram(s)

mi mile(s)

$\mathrm{mL}$ milliliter(s)

$\mathrm{pCi}$ picocurie(s)

qt quart(s)

rad radiation absorbed dose

yr year(s) 


\begin{tabular}{|c|c|c|}
\hline Multiply & By & To Obtain \\
\hline \multicolumn{3}{|l|}{ English/Metric Equivalents } \\
\hline acres & 0.4047 & hectares (ha) \\
\hline cubic feet $\left(\mathrm{ft}^{3}\right)$ & 0.02832 & cubic meters $\left(\mathrm{m}^{3}\right)$ \\
\hline cubic yards $\left(\mathrm{yd}^{3}\right)$ & 0.7646 & cubic meters $\left(\mathrm{m}^{3}\right)$ \\
\hline degrees Fahrenheit $\left({ }^{\circ} \mathrm{F}\right)-32$ & 0.5555 & degrees Celsius $\left({ }^{\circ} \mathrm{C}\right)$ \\
\hline feet $(\mathrm{ft})$ & 0.3048 & meters $(\mathrm{m})$ \\
\hline gallons (gal) & 3.785 & liters $(\mathrm{L})$ \\
\hline gallons (gal) & 0.003785 & cubic meters $\left(\mathrm{m}^{3}\right)$ \\
\hline inches (in.) & 2.540 & centimeters $(\mathrm{cm})$ \\
\hline miles (mi) & 1.609 & kilometers (km) \\
\hline pounds (lb) & 0.4536 & kilograms (kg) \\
\hline short tons (tons) & 907.2 & kilograms (kg) \\
\hline short tons (tons) & 0.9072 & metric tons $(t)$ \\
\hline square feet $\left(\mathrm{ft}^{2}\right)$ & 0.09290 & square meters $\left(\mathrm{m}^{2}\right)$ \\
\hline square yards $\left(\mathrm{yd}^{2}\right)$ & 0.8361 & square meters $\left(\mathrm{m}^{2}\right)$ \\
\hline square miles $\left(\mathrm{mi}^{2}\right)$ & 2.590 & square kilometers $\left(\mathrm{km}^{2}\right)$ \\
\hline yards (yd) & 0.9144 & meters $(\mathrm{m})$ \\
\hline \multicolumn{3}{|l|}{ Metric/English Equivalents } \\
\hline centimeters $(\mathrm{cm})$ & 0.3937 & inches (in.) \\
\hline cubic meters $\left(\mathrm{m}^{3}\right)$ & 35.31 & cubic feet $\left(\mathrm{ft}^{3}\right)$ \\
\hline cubic meters $\left(\mathrm{m}^{3}\right)$ & 1.308 & cubic yards $\left(\mathrm{yd}^{3}\right)$ \\
\hline cubic meters $\left(\mathrm{m}^{3}\right)$ & 264.2 & gallons (gal) \\
\hline degrees Celsius $\left({ }^{\circ} \mathrm{C}\right)+17.78$ & 1.8 & degrees Fahrenheit $\left({ }^{\circ} \mathrm{F}\right)$ \\
\hline hectares (ha) & 2.471 & acres \\
\hline kilograms (kg) & 2.205 & pounds (lb) \\
\hline kilograms $(\mathrm{kg})$ & 0.001102 & short tons (tons) \\
\hline kilometers $(\mathrm{km})$ & 0.6214 & miles (mi) \\
\hline liters $(\mathrm{L})$ & 0.2642 & gallons (gal) \\
\hline meters (m) & 3.281 & feet (ft) \\
\hline meters $(\mathrm{m})$ & 1.094 & yards $(y d)$ \\
\hline metric tons $(t)$ & 1.102 & short tons (tons) \\
\hline square kilometers $\left(\mathrm{km}^{2}\right)$ & 0.3861 & square miles $\left(\mathrm{mi}^{2}\right)$ \\
\hline square meters $\left(\mathrm{m}^{2}\right)$ & 10.76 & square feet $\left(\mathrm{ft}^{2}\right)$ \\
\hline square meters $\left(\mathrm{m}^{2}\right)$ & 1.196 & square yards $\left(\mathrm{yd}^{2}\right)$ \\
\hline
\end{tabular}




\section{INTRODUCTION}

The U.S. Department of Energy (DOE) and the U.S. Department of the Army (DA) are evaluating conditions in groundwater and springs at the DOE chemical plant area and the DA ordnance works area near Weldon Spring, Missouri. The two areas are located in St. Charles County, about $48 \mathrm{~km}$ (30 mi) west of St. Louis (Figure 1.1). The 88-ha (217-acre) chemical plant area is chemically and radioactively contaminated as a result of uranium-processing activities conducted by the U.S. Atomic Energy Commission in the 1950s and 1960s and explosivesproduction activities conducted by the U.S. Army (Army) in the 1940s. The 6,974-ha (17,232-acre) ordnance works area is primarily chemically contaminated as a result of trinitrotoluene (TNT) and dinitrotoluene (DNT) manufacturing activities during World War II.

This baseline risk assessment (BRA) is being conducted as part of the remedial investigation/feasibility study (RIFS) required under the Comprehensive Environmental Response, Compensation, and Liability Act (CERCLA) of 1980, as amended. The purpose of the BRA is to evaluate potential human health and ecological impacts from contamination associated with the groundwater operable units (GWOUs) of the chemical plant area and ordnance works area. An RU/FS work plan issued jointly in 1995 by the DOE and DA (DOE 1995) analyzed existing conditions at the GWOUs. The work plan included a conceptual hydrogeological model based on data available when the report was prepared; this model indicated that the aquifer of concern is common to both areas. Hence, to optimize further data collection and interpretation efforts, the DOE and DA have decided to conduct a joint RI/BRA.

Characterization data obtained from the chemical plant area wells indicate that uranium is present at levels slightly higher than background, with a few concentrations exceeding the proposed U.S. Environmental Protection Agency (EPA) maximum contaminant level (MCL) of $20 \mu \mathrm{g} / \mathrm{L}$ (EPA 1996c). Concentrations of other radionuclides (e.g., radium and thorium) were measured at background levels and were eliminated from further consideration (DOE 1995). Chemical contaminants identified in wells at the chemical plant area and ordnance works area include nitroaromatic compounds, metals, and inorganic anions. Trichloroethylene (TCE) and 1,2-dichloroethylene $(1,2-D C E)$ have been detected recently in a few wells near the raffinate pits at the chemical plant.

\subsection{SCOPE OF THE BASELINE RISK ASSESSMENT}

The human health component of this BRA provides risk estimates for exposure to groundwater and spring water. The focus of the groundwater assessment is the shallow aquifer system represented by wells completed in the Burlington-Keokuk Limestone and Fern Glen Formation. Previous evaluations have indicated that the potential for contaminated water in the shallow aquifer to enter the deep aquifer is small, and the time required for water to travel this distance is measured in hundreds of years (Kleeschulte 1991). In addition, the potentiometric surface of the deep bedrock 


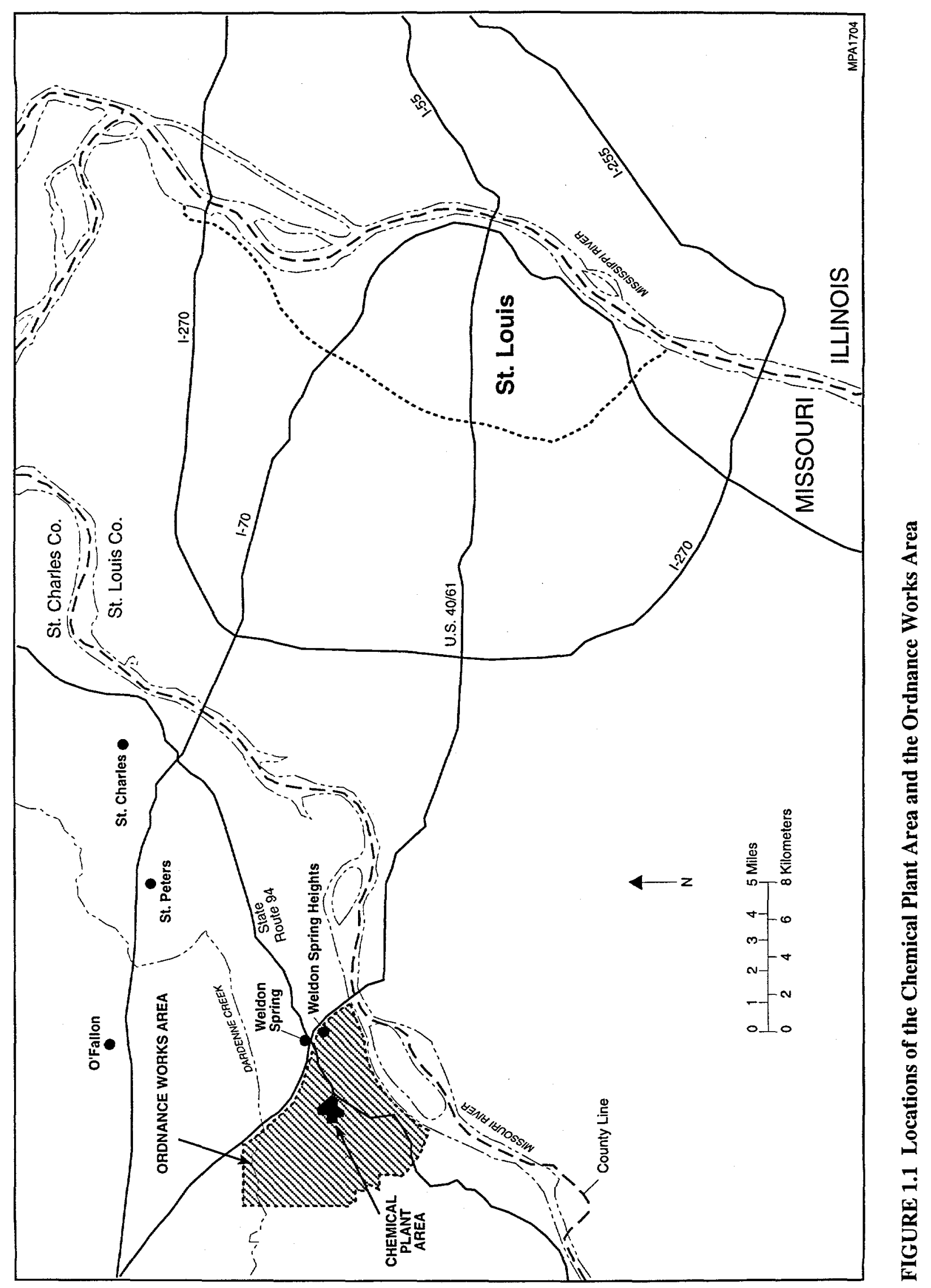


aquifer is significantly lower than those of the shallow and middle aquifers, which indicates a limited hydrogeologic connection between the deep and upper aquifers (DOE and DA 1997).

Both the DOE and DA have previously evaluated conditions at the area springs, including Burgermeister Spring. DOE is currently addressing contamination in springs at the Southeast Drainage; separate documentation has been prepared by DOE to support decision making for this drainage (DOE 1997). The results of earlier evaluations for the area springs indicated that the potential human health risk from spring water is minimal; the estimated risks were lower than the acceptable risk range recommended by the EPA. Also, contaminant concentrations and the potential risk from sediments were lower than those in area spring water, except at the Southeast Drainage. These results are presented in the BRA reports supporting the operable units that addressed soil and structural contamination at the chemical plant area (DOE 1992) and at the ordnance works area (International Technology [IT] Corporation 1993b). However, in May and August of 1995, more recent spring water data were collected from selected springs as part of the joint DOE/DA sampling effort. The 15 springs sampled, including SP-5303 at the Southeast Drainage, were selected because they were considered to be locations that receive groundwater discharge. An assessment of potential human health and ecological impacts from these springs is included in this report to provide an updated evaluation incorporating these recent data.

The ecological risk assessment addresses impacts to aquatic and terrestrial biota from groundwater that discharges to the surface at springs; the assessment was conducted in accordance with EPA guidance (EPA 1992b). Risk estimates to aquatic biota were based on direct exposure to contaminated media, whereas risks to terrestrial biota were based on modeled uptake of contaminants via direct ingestion of surface water. The ecological risk assessment also evaluates the conditions of aquatic biota and habitats associated with Burgermeister Spring and includes measurements of the toxicity to aquatic biota of surface water and sediment from this spring. Burgermeister Spring receives discharge of groundwater originating from the chemical plant and ordnance works areas, and concentrations of some contaminants are as high or higher than concentrations from most other springs in the area. Furthermore, Burgermeister Spring and downstream areas provide more permanent habitat for aquatic biota than most of the other springs in the area and thus likely support a more diverse and abundant aquatic biota than the other springs. Therefore, maximum environmental impacts could be associated with contaminants in the Burgermeister Spring system. Higher concentrations of some contaminants have been reported from springs in the 5300 drainage, which provide more permanent habitat than most other springs in the area. However, springs in the 5300 drainage were not evaluated in this ecological risk assessment because ecological risks associated with this drainage basin have been evaluated previously and are discussed in DOE (1996).

Risk estimates for current and future land use projections were conducted in accordance with EPA guidance (EPA 1989b-c). Current land uses at both the chemical plant area and the ordnance works area do not include use of groundwater for drinking; however, access to springs is possible. Future land uses at both areas would be expected to be similar to current land uses. To address current and likely future potential exposure to springs in these two areas, a recreational 
visitor scenario was developed and calculated. Access to groundwater was assumed for a hypothetical resident scenario; the risk from groundwater for a future resident was calculated to provide information representing potential upper-bound risk.

Although the main scope of this report addresses potential risk from groundwater and spring water contaminants, cumulative risks for the future recreational visitor and residential scenarios, incorporating projected exposures to other site media (i.e., soil), are discussed in Chapter 5. Risk for soil was assessed and presented in reports prepared to support cleanup of soil and structural contamination at the chemical plant area (DOE 1992) and the ordnance works area (IT Corporation 1993b).

\subsection{PURPOSE OF THIS BASELINE RISK ASSESSMENT}

This BRA provides a baseline of potential human health and ecological impacts for the GWOUs at the chemical plant area and ordnance works area. It estimates the magnitude of potential health risks and environmental impacts that would be associated with GWOU contaminants if no remedial action were taken. In addition, the risk estimates presented in this BRA serve as a baseline for comparison with the protectiveness of cleanup alternatives discussed in upcoming RI/FS reports.

\subsection{ORGANIZATION OF THE REPORT}

The assessment approach followed in this report is consistent with the approach recommended in EPA guidance (EPA 1989b-c). The report is organized as follows:

- Chapter 2 - Description of data sources, data interpretation, and evaluation procedures, and identification of the contaminants of potential concern (COPCs).

- Chapter 3 - Discussion of the development of the human health exposure scenarios to depict current and future land uses, the ecological exposure assessment, and the derivation of exposure point concentrations and intakes.

- Chapter 4 - Brief discussion of the toxicities of the COPCs.

- Chapter 5 - Results of the human health risk assessment and accompanying rationale.

- Chapter 6 - Results of the ecological risk assessment.

- Chapter 7 - Summary of human health and ecological impacts.

- Chapter 8 - List of references cited. 


\section{IDENTIFICATION OF CONTAMINANTS OF CONCERN}

Information relevant to collecting and evaluating data for the human health and ecological risk assessments in this BRA is summarized in this chapter. General background information for the GWOUs, including origin of contamination, is presented in the work plan (DOE 1995). Data summaries and detailed descriptions of data collection efforts are presented in the RI report (DOE and DA 1997).

A considerable amount of data was available at the initial (work plan) phases of the RI/FS, allowing for a more conclusive interpretation of the data. As a result, potential contaminants were identified, which were evaluated further on the basis of data collected from the joint sampling performed by the DOE and DA in May and August of 1995. The COPCs that were carried through the calculations for the human health component of this BRA are identified in the RI on the basis of comparison to background levels of naturally occurring constituents. The process performed for identifying contaminants of ecological concern (COECs) is discussed in Section 2.2.

\subsection{DATA CONSIDERATIONS}

The data evaluated for use in this risk assessment are presented in the RI report (DOE and DA 1997). The quantity of data was sufficient to develop an adequate statistical base for use in the risk assessment calculations. The quality of the data is discussed in Section 7 of the RI report and was also considered sufficient for use in this risk assessment.

Monitoring results for the contaminants from 155 wells included in the monitoring networks at the chemical plant area and ordnance works area are presented in Chapter 4 of the RI report. The locations of these wells are shown in Figure 2.1. Monitoring results from five other wells in the ordnance works network - wells MWS-23, MWS-111, MWD-105, MWS-108, and MWD-109 - are presented in the RI as background data (DOE and DA 1997). Wells within this network have been categorized as deep, overburden, weathered, or unweathered wells, as discussed in the RI. Because it is likely that any potential future consumptive-use well would draw water from all of these units, data for all wells were considered in the human health risk calculations. The RI also presents results from in-situ groundwater sampling at six locations at or near the Southeast Drainage. To aid in better delineation of the extent of uranium contamination in the area, a monitoring well was installed recently (May 1997); one round of sampling and analysis has been performed to date. Because of the preliminary nature of these data, a qualitative discussion of the risk associated with the detected contaminant levels is included in Chapter 5 (Sections 5.3 and 5.4) of this BRA.

All groundwater and spring water data collected by the DOE and DA were considered for use in this assessment, except those that were qualified as invalid and identified as "rejected" in the databases. Of the approximately 50,000 discrete records available to determine groundwater quality 
in the GWOUs, approximately 200 records (less than $0.5 \%$ ) were rejected because of laboratory quality assurance/quality control concerns.

Data for assessment of spring water are available from the DOE, DA, and U.S. Geological Survey (USGS). The DOE data cover the period from late 1987 through the first quarter of 1995; the DA data include data reported for sampling rounds 2 and 4 to 16 covering the period November 1989 through February 1995 (IT Corporation 1992, 1993a-f, 1994a-d, 1995a-b); and the USGS data are for nitroaromatic compounds from eight springs, including Burgermeister Spring. Data for 15 springs are also available from the joint sampling rounds performed by the DOE and DA in 1995. The locations of these springs are shown in Figure 2.1 .

\subsection{DATA EVALUATION}

A subset of constituents was selected from the potential contaminants identified in the RI report (DOE and DA 1997) to focus the risk assessment on only those contaminants considered to be significant contributors to overall risks. These data evaluation procedures have been recommended by the EPA $(1989 \mathrm{~b}, 1993)$ to select the human health COPCs and the ecological COECs. However, all contaminants identified in the RI were considered to be COPCs for the human health assessment and were carried through the risk calculations presented in the remainder of this report. In the RI, groundwater and surface water data were compared with background levels, and those constituents exceeding background levels were identified as site contaminants, as follows:

- Metals: antimony, cadmium, iron, lithium, manganese, mercury, molybdenum, silver, and uranium;

- Inorganic anions: chloride, nitrate- $\mathrm{N}$, and sulfate;

- Organic compounds: 1,3,5-trinitrobenzene (1,3,5-TNB), 1,3-dinitrobenzene (1,3-DNB), 2,4,6-trinitrotoluene (2,4,6-TNT), 2,4-dinitrotoluene (2,4-DNT), 2,6-dinitrotoluene (2,6-DNT), 2-amino-4,6-dinitrotoluene (2-amino-4,6-DNT), 4-amino-2,6-dinitrotoluene (4-amino-2,6-DNT), m-nitrotoluene, o-nitrotoluene, p-nitrotoluene, nitrobenzene, trichloroethylene (TCE), and 1,2-dichloroethylene (1,2-DCE).

Each contaminant has been identified as either a spring water contaminant, groundwater contaminant, or both (see Table 2.1).

Uranium was evaluated as both a radioactive and a chemical contaminant. The concentrations of uranium in groundwater and spring water are generally reported in units of picocuries of total uranium (i.e, the sum of the activities of uranium-238, uranium-235, and uranium-234) per liter of water $(\mathrm{pCi} / \mathrm{L})$. Because the slope factors for these three radionuclides are essentially identical (see

Section 4.3.1), it was not necessary to know the exact mix of uranium isotopes to calculate the 


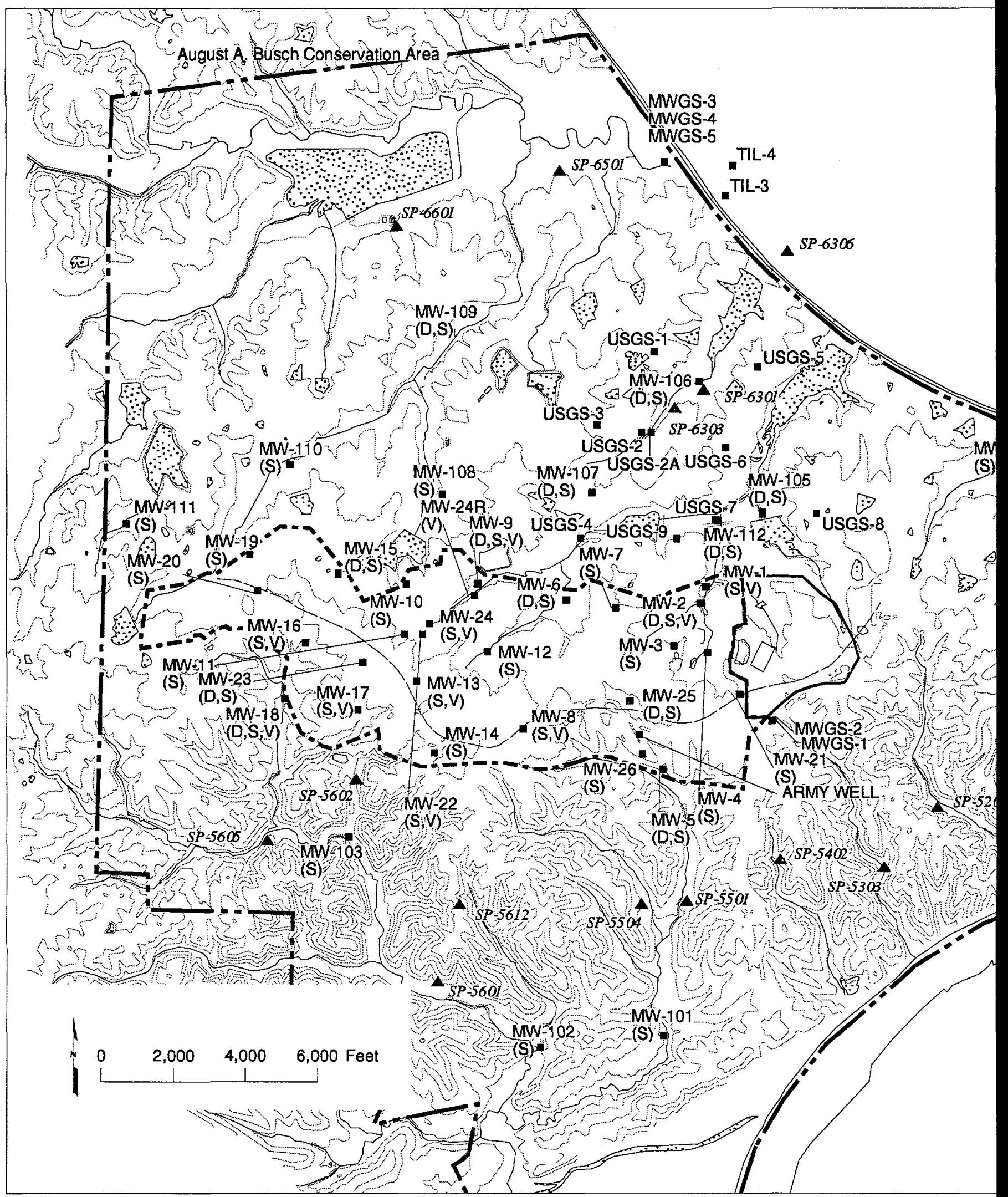

FIGURE 2.1 Location of Monitoring Wells and Springs at the Chemical Plant and Ordnance Works Area 
To simplify the analyses and add some conservatism to the risk results, it was assumed that the uranium isotopes are present in groundwater and spring water in the same concentrations as they are in chemical plant area soil. Measured activities at each sampling location were used to calculate the radiological risk, and these activities were converted to mass concentrations using a conversion factor of $0.0015 \mathrm{mg} / \mathrm{pCi}$ of total uranium. This conversion factor was obtained from the specific activities of the three uranium isotopes ${ }^{1}$ (assumed to be present in the ratios identified above). Because uranium-234 and uranium-235 both have higher specific activities than uranium-238 (due to their shorter half-lives), this approach tends to overestimate the mass concentration of uranium in those instances where uranium-234 (and possibly uranium-235) has a higher activity ratio (relative to uranium-238) than in chemical plant area soil. In cases where the uranium-234 and uranium-238 concentrations are essentially the same, this approach provides an accurate estimate of the mass concentration. In no case was the activity of uranium-234 less than that of uranium-238. This approach for estimating the mass concentration of uranium at all sampling locations for groundwater and spring water provides, in a consistent manner using all available data, a realistic yet somewhat conservative estimate of the chemical risk associated with uranium intake.

The COECs in surface water from all spring locations and in sediments from Burgermeister Spring were identified by comparing the reported concentrations (see Table 2.1) with several criteria, including background concentrations and screening benchmark values considered to be protective of aquatic biota, as outlined in EPA guidance (EPA 1989c, 1992b). All contaminants detected in surface water were evaluated in the risk assessment for terrestrial biota. The screening process also considered the contaminant's ability to bioaccumulate and the contaminant's importance as a microor macronutrient.

The screening for COECs proceeded in three steps. First, the screening considered only those contaminants detected in samples from all springs and proximate downstream locations for which data were available. Second, the $95 \%$ upper confidence limit (UCL) concentration for each metal and inorganic ion was compared with the corresponding 95\% UCL concentration detected in the background monitoring wells completed in the weathered zone of the Burlington-Keokuk. Because spring water is groundwater that is discharging to the surface, use of the groundwater data from the background monitoring wells as background spring data is justified. A contaminant was retained for further screening when the reported $95 \%$ UCL spring water concentration exceeded the 95\% UCL background level. Because nitroaromatic compounds do not occur naturally, background concentrations of these were assumed to be zero. Third, for those contaminants that did exceed background levels, the $95 \%$ UCL concentrations were then compared with screening values, and constituents present at concentrations exceeding screening concentrations were retained as final COECs for further evaluation in the ecological risk assessment. Surface water concentrations were compared with either the chronic effects value of the EPA ambient water quality criteria (AWQC) for protection of aquatic biota (EPA 1986) or the AWQC acute effects value if a chronic value was

1 The specific activities for uranium-234, uranium-235, and uranium-238 are $6.320 \times 10^{-3} \mathrm{Ci} / \mathrm{g}, 2.186 \times 10^{-6} \mathrm{Ci} / \mathrm{g}$, and $3.400 \times 10^{-7} \mathrm{Ci} / \mathrm{g}$, respectively; the specific activity for natural uranium is $6.77 \times 10^{-7} \mathrm{Ci} / \mathrm{g}$ (Brodsky 1996). 
unavailable. Other sources of screening values for surface waters included EPA Region III screening guidance (EPA 1995a), Suter and Tsao (1996), Eisler (1985), and the open scientific literature. Sediment screening values were obtained from EPA ecotox threshold values (EPA 1996a), Long and Morgan (1990), Hull and Suter (1994), EPA Region III screening guidance (EPA 1995a), and the scientific literature. Results of the screening process and the COECs are given in Tables 2.2 and 2.3.

TABLE 2.2 Screening of Sediment from the Burgermeister Spring System to Identify COECs

\begin{tabular}{|c|c|c|c|c|c|}
\hline Contaminant & $\begin{array}{l}\text { Detection } \\
\text { Frequency }\end{array}$ & $\begin{array}{c}\text { Concentration } \\
\text { Range } \\
(\mathrm{mg} / \mathrm{kg})\end{array}$ & $\begin{array}{c}\text { Background } \\
\text { Concentration } \\
(\mathrm{mg} / \mathrm{kg})\end{array}$ & $\begin{array}{c}\text { Screening } \\
\text { Concentration } \\
(\mathrm{mg} / \mathrm{kg})\end{array}$ & $\begin{array}{c}\text { Retain } \\
\text { as COEC? }\end{array}$ \\
\hline \multicolumn{6}{|l|}{ Metals } \\
\hline Arsenic & $8 / 8$ & $3.1-43$ & $5.7-16$ & $8.2^{\mathrm{b}}$ & Yes \\
\hline Chromium & $8 / 8$ & $12-48$ & $16-32$ & $81^{b}$ & No \\
\hline Lead & $8 / 8$ & $12-110$ & $4.9-28$ & $47^{b}$ & Yes \\
\hline Mercury & $0 / 8$ & $-^{c}$ & - & $0.15^{\mathrm{b}}$ & No \\
\hline Selenium & $1 / 8$ & 0.96 & 0.54 & $N A^{c}$ & Yes \\
\hline Silver & $3 / 8$ & $1.6-1.7$ & 1.1 & $1.0^{\mathrm{d}}$ & Yes \\
\hline Uranium, total & $8 / 8$ & $1.4-100$ & $1.6-2.6$ & NA & Yes \\
\hline \multicolumn{6}{|l|}{ Inorganic anion } \\
\hline Nitrate-N & $4 / 8$ & $1.0-5.0$ & 0.99 & NA & Yes \\
\hline \multicolumn{6}{|c|}{ Nitroaromatic compounds } \\
\hline $1,3,5-\mathrm{TNB}$ & $0 / 8$ & - & - & $0.30^{\mathrm{e}}$ & No \\
\hline 1,3-DNB & $0 / 8$ & - & - & $1.2^{\mathrm{e}}$ & No \\
\hline $2,4,6-\mathrm{TNT}$ & $0 / 8$ & - & - & $13^{\mathrm{e}}$ & No \\
\hline 2,4-DNT & $0 / 8$ & - & - & NA & No \\
\hline Nitrobenzene & $0 / 4$ & - & - & NA & No \\
\hline
\end{tabular}

a Background concentrations are those reported for the Busch Conservation Area in the chemical plant area baseline assessment (DOE 1992).

b Screening value is EPA ecotox threshold value (EPA 1996a).

c A hyphen (-) indicates the contaminant was not detected; $\mathrm{NA}=$ screening value not available.

d Benchmark value from Hull and Suter (1994).

e Screening value from Talmage and Opresko (1996). 
TABLE 2.3 Screening of Surface Water from Springs in the Chemical Plant Area and the Ordnance Works Area to Identify COECs

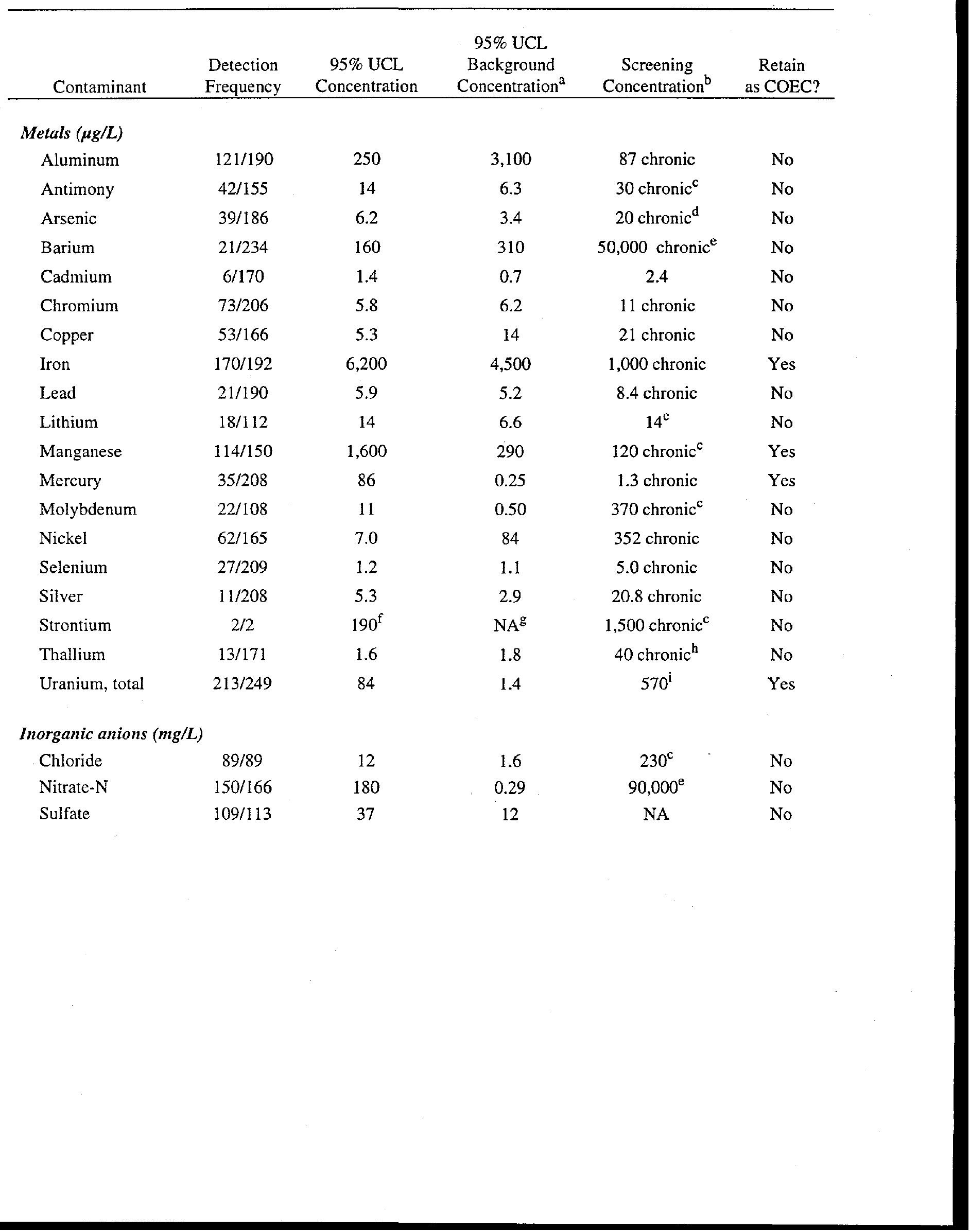


TABLE 2.3 (Cont.)

\begin{tabular}{lccccc}
\hline \multicolumn{1}{c}{ Contaminant } & $\begin{array}{c}\text { Detection } \\
\text { Frequency }\end{array}$ & $\begin{array}{c}95 \% \text { UCL } \\
\text { Concentration }\end{array}$ & $\begin{array}{c}\text { 95\% UCL } \\
\text { Background } \\
\text { Concentration }^{\text {a }}\end{array}$ & $\begin{array}{c}\text { Screening } \\
\text { Concentration }^{\mathrm{b}}\end{array}$ & $\begin{array}{c}\text { Retain } \\
\text { as COEC? }\end{array}$ \\
\hline $\begin{array}{l}\text { Nitroaromatic } \\
\text { compounds }(\mu g / L)\end{array}$ & & & & & \\
1,3,5-TNB & $61 / 278$ & 0.56 & $-\mathrm{g}$ & 14 chronic $^{\mathrm{j}}$ & No \\
1,3-DNB & $17 / 276$ & 0.033 & - & 30 chronic $^{\mathrm{j}}$ & No \\
2,4,6-TNT & $136 / 279$ & 8.1 & - & 130 chronic $^{\mathrm{j}}$ & No \\
2,4-DNT & $81 / 279$ & 0.20 & - & 230 chronic $^{\mathrm{k}}$ & No \\
2,6-DNT & $111 / 277$ & 0.49 & - & NA $^{2}$ & Yes \\
Nitrotoluene & $3 / 380$ & 0.0023 & - & NA & Yes \\
2-Amino-4,6-DNT & $68 / 85$ & 2.0 & - & $0.02^{\mathrm{c}}$ & Yes \\
4-Amino-2,6-DNT & $67 / 85$ & 2.8 & - & NA & Yes \\
Nitrobenzene & $11 / 278$ & 0.027 & - & NA & Yes \\
\hline
\end{tabular}

a Background concentrations are the concentrations measured in samples collected from background monitoring wells.

b Screening values are EPA (1986) AWQC unless otherwise noted. All hardness-dependent values were calculated using hardness $=258.9 \mathrm{mg}$ equivalent calcium carbonate per liter.

c Screening value from Suter and Tsao (1996).

d State of Missouri water quality criteria for the protection of aquatic life (Missouri Department of Natural Resources 1992).

e EPA (1986) identifies the concentration as a potentially "safe" maximum concentration; no AWQC is identified.

f Because of the small sample size, it was not possible to calculate a $95 \%$ UCL value; the reported value is the maximum reported concentration.

g NA = not available; a hyphen (-) indicates the contaminant was not detected;

h EPA (1986) states that insufficient data are available to develop AWQC; screening value is lowestobserved-effects level identified in EPA (1986).

i No AWQC available; screening concentration is lowest reported concentration to be chemotoxic to aquatic biota (Poston et al. 1984).

j Secondary chronic value (Talmage and Opresko 1996).

k EPA Region III screening value (EPA 1995a).

$l$ Includes o-nitrotoluene, m-nitrotoluene, and p-nitrotoluene. 


\section{EXPOSURE AND EFFECTS ASSESSMENT}

The former ordnance works area has been divided into several contiguous areas with different land uses (Figure 3.1). The 670-ha (1,655-acre) Weldon Spring Training Area is adjacent to the 88-ha (217-acre) chemical plant area. Both areas are fenced, and access by the general public is restricted. Portions of the training area are currently used for field training and outdoor drilling by the U.S. Army Reserve, the Missouri Army National Guard, and other military and police units. An estimated 3,300 local Army reservists and 3,400 other reserve troops use the training area each year. The Army intends to continue using the training area for similar training activities in the future. Most of the remaining portions of the ordnance works area have been converted into two conservation areas: the 2,977-ha (7,356-acre) Weldon Spring Conservation Area and the 2,828-ha (6,987-acre) August A. Busch Memorial Conservation Area. These areas are managed by the Missouri Department of Conservation and are open to the public throughout the year for recreational use. Future land uses for the ordnance works area and chemical plant area are expected to remain similar to current land uses, except that a disposal cell currently under construction will occupy up to one-third of the chemical plant area.

\subsection{IDENTIFICATION OF EXPOSURE PATHWAYS}

Potential human and biotic exposure pathways were identified for this study on the basis of the following factors:

- Locations of contaminated source areas, types of contaminants found at the source areas, and potential mechanisms of contaminant release from those areas;

- Likely fate and transport of the contaminants within or between environmental media;

- Estimated concentrations of contaminants at points of potential human and biota contact (i.e., exposure points) and the associated probable routes of human and biota exposure (e.g., ingestion); and

- Completeness of each exposure pathway - that is, the presence of a source and a mechanism of contaminant release, an environmental transport medium, a point of human and biota contact with the contaminated source or medium, and a route of human and biota exposure at that point.

All of these factors were considered in developing the conceptual site exposure model presented in Figure 3.2. Detailed discussion regarding sources, nature and extent of contamination, and fate and transport of contaminants is presented in the RI (DOE and DA 1997). 


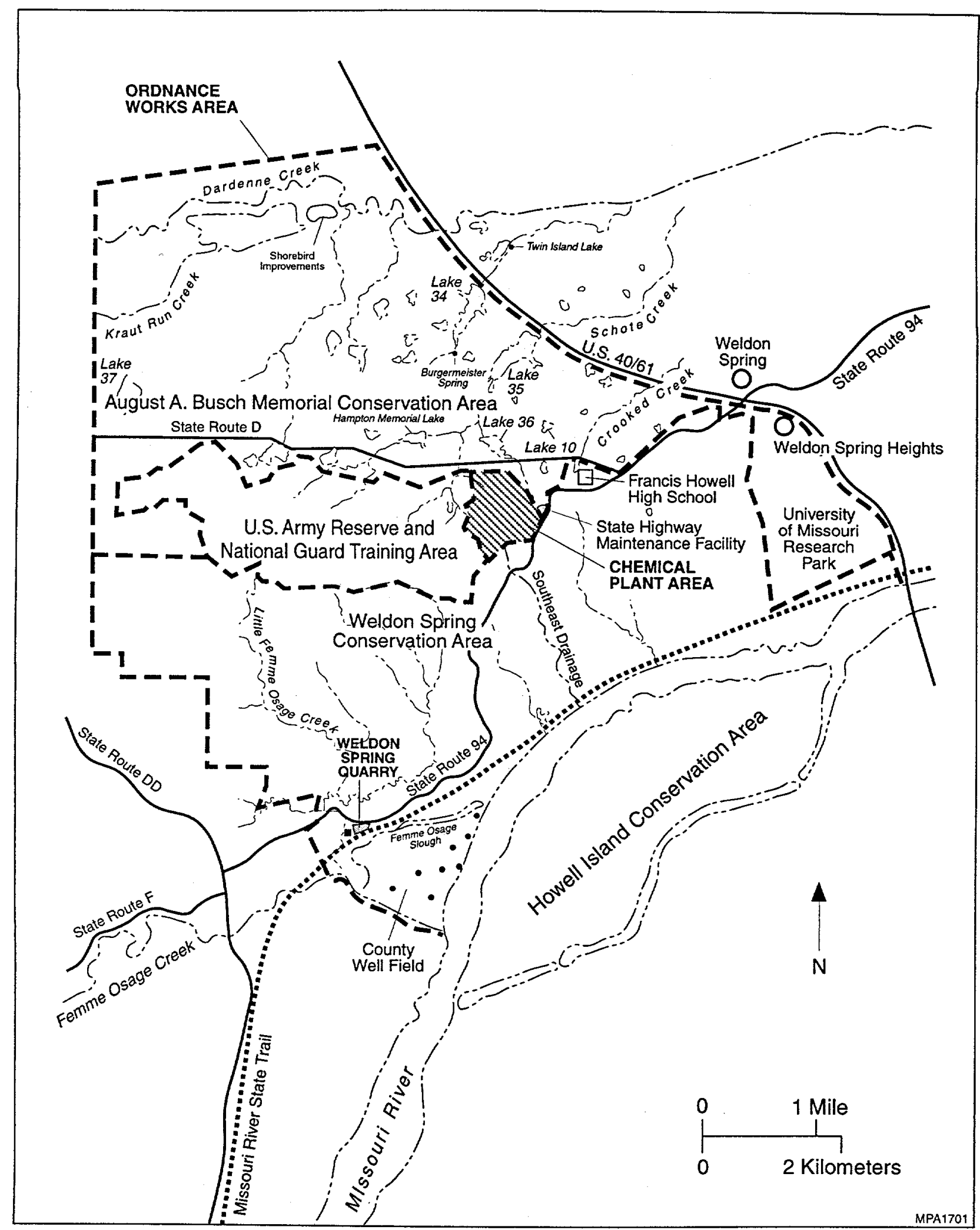

FIGURE 3.1 Map of the Chemical Plant Area and Ordnance Works Area and Immediate Vicinity 


\subsubsection{Human Health Pathways}

The principal route of exposure for a human receptor is considered to be ingestion of spring water. Dermal exposure to spring water was also calculated, although this exposure pathway would be less significant based on the limited area and depth of most springs. Because of the small size of the springs and the very low levels of contamination measured in the spring sediments, the potential for dermal contact with, or ingestion of, sediment is considered to be low. Inhalation was not identified as a pathway of concern because of the absence of volatile organic compounds, radon, and airborne particulates. External gamma radiation was also eliminated as a pathway of concern because of the small size of the springs and the very low levels of uranium in the sediment. The water cover over the sediment also acts to attenuate the low-level gamma radiation.

Under current land uses, the most likely receptor would be a recreational visitor who might be exposed to contaminated discharge water at one of the springs. Army reservists and a full-time site caretaker of the ordnance works area were also considered as potential receptors; however, these scenarios were not evaluated. There are no potential pathways of exposure for the reservist because no active springs are located in the Army training area and municipal water is available at the tap. Similarly, the potential for the site caretaker to come in contact with contaminated groundwater and spring water is unlikely because of the availability of municipal water. The potential risk to a reservist who might venture outside the fenced training area and drink spring water is covered by the calculations performed for the recreational visitor (however, one may assume that if a reservist visited parts of the ordnance works area other than the training area, he would take a drinking water supply with him). It was considered reasonable for reservists to train at the training area two to three weekends (about 6 days) per year. If these same reservists also spent their yearly retreat training of 2 weeks there, the frequency of exposure would extend to about 20 days, which is the same as the exposure frequency assumed for a recreational visitor.

Because future land-use conditions are expected to be similar to current conditions, the most likely receptor was also assumed to be a recreational visitor. The Army intends to continue using the training area for training activities in the future. The 89th Regional Support Command, U.S. Army Reserve, has developed plans to construct a training center at the Weldon Spring Training Area. This facility would contain headquarters for several reserve units with about 30 full-time personnel. The units headquartered at the facility would conduct drills on assigned weekends and evenings at the facility and the training area. The chemical plant area is currently being remediated, and all site waste will be disposed of in an engineered disposal cell constructed on-site. The cell is estimated to occupy approximately one-third of the chemical plant area.

The August A. Busch Memorial Conservation Area and the Weldon Spring Conservation Area, which occupy a large portion of the former ordnance works area, are managed by the Missouri Department of Conservation and are open throughout the year for recreational use. These areas are extensively used, as indicated by the estimated 1,200,000 visitors each year (Crigler 1992). 


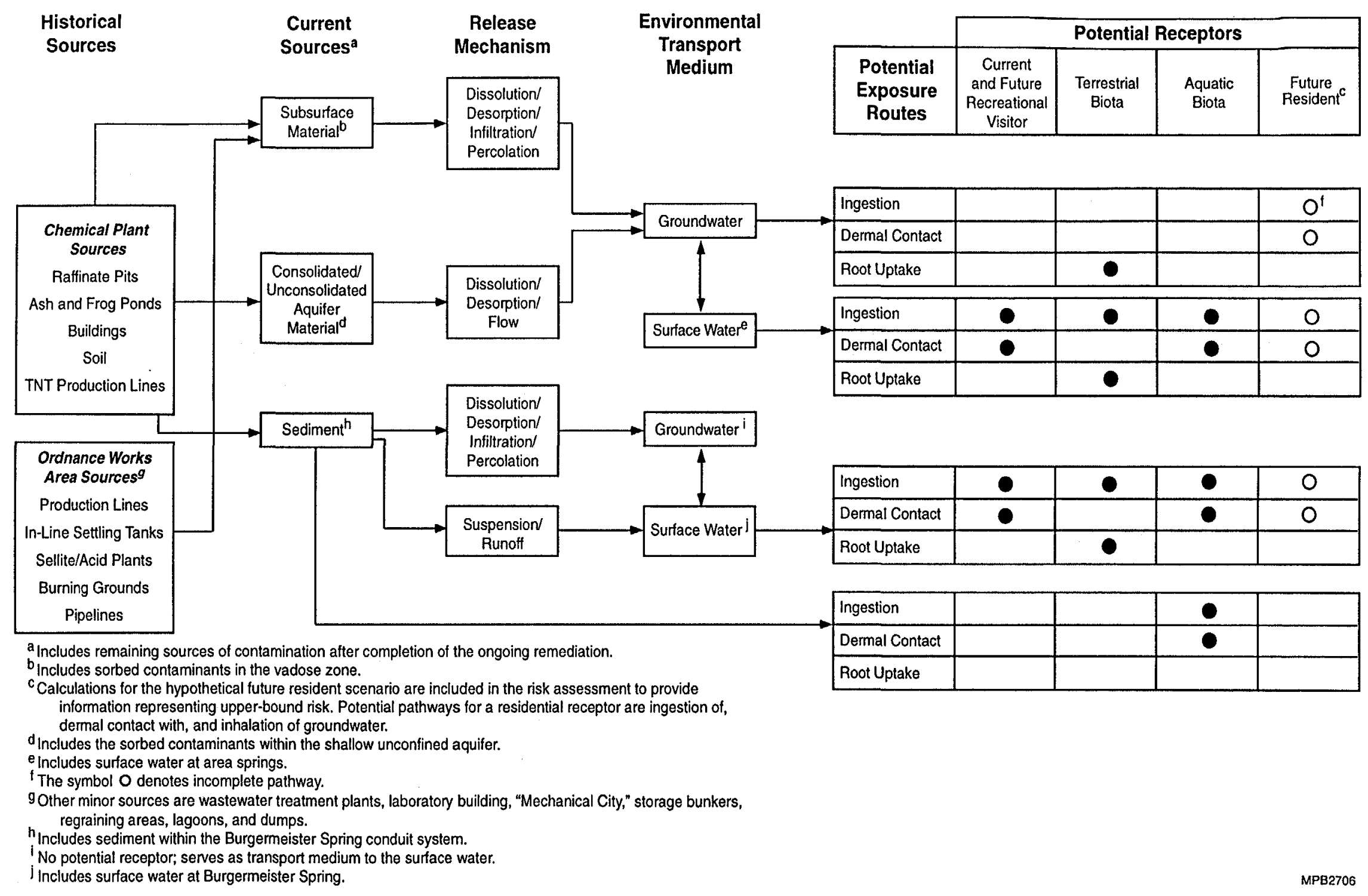

FIGURE 3.2 Conceptual Site Exposure Model for the Chemical Plant Area and Ordnance Works Area 
Current land uses in the vicinity also include a state highway maintenance facility east of the chemical plant area and a private housing development known as Weldon Spring Heights. Both the maintenance facility and the housing development receive their water from the St. Charles County municipal water supply. Francis Howell High School, located about $1 \mathrm{~km}(0.6 \mathrm{mi})$ east of the chemical plant area, also obtains its water from St. Charles County.

Forty-five old wells were identified on the ordnance works area as a result of a review of archival records from state files and interviews with persons familiar with the site. Many of these private wells were open to the deeper bedrock aquifers (i.e., Kimmswick and St. Peter) to obtain sufficient well yields. Although some of these private wells were open to the shallow aquifer, to obtain sufficient yield they were open throughout the entire shallow aquifer (including all or part of the Fern Glen), rather than only the upper weathered part of the Burlington-Keokuk.

Due to the low transmissivity and low yield of the shallow aquifer, a future resident would likely screen a private well in the deeper, more productive aquifers or, because of the $24-\mathrm{m}$ ( $80-\mathrm{ft}$ ) casing requirement, the well would be open to a larger portion of the shallow aquifer (rather than only the upper weathered unit of the Burlington-Keokuk). The 80-ft casing requirement would, of itself, not preclude using water from the shallow groundwater system. Use of a longer screen would improve the quality of the pumped water because of mixing with less contaminated water (the contamination decreases with depth). In 1989, pumping tests for the shallow aquifer at the chemical plant area indicated a maximum sustainable pump rate of 0.3 gallons per minute. Even with an extended casing, well yields would barely support the daily use of a family. However, the low yields could be increased by installing a cistern and replenishing the cistern from the groundwater; this approach would supply a sufficient amount of water to support a typical household. Multiple singlefamily housing units in a future subdivision development in the area would most likely receive water from a municipal water supplier. This water would be obtained from deeper formations such as the Kimmswick or St. Peter formations.

Risk calculations were also performed for a hypothetical future resident scenario because this scenario would provide potential upper-bound risk information to aid in risk management decision making for groundwater. Pathways evaluated included ingestion and dermal contact through showering. The inhalation pathway was evaluated only for TCE. Similar calculations for recreational use of the groundwater would result in hazard indices or risks of approximately one-hundredth of those estimated for the hypothetical future resident. Exposure parameters for the human health receptors are summarized in Table 3.1.

1 All tables in this chapter have been placed at the of the text (Section 3.4.5). 


\subsubsection{Ecological Effects}

Ecological health effects were also evaluated as part of the exposure assessment. Because of the nature of the contamination, risks to ecological resources would be related primarily to direct contact and ingestion of surface water and sediment originating at a spring; therefore, the ecological risk assessment focused primarily on (1) aquatic biota inhabiting a spring and immediate downstream habitats and (2) terrestrial biota drinking from a spring and downstream locations.

For aquatic biota, the exposure scenario consists of direct exposure to contaminated spring water and sediment. Risk calculations were performed using the 95\% UCL concentrations for the spring water and the maximum contaminant concentrations for the sediment. Burgermeister Spring and its immediate downstream habitats was chosen as the exposure area for all risk determinations in this study. Although some risks to aquatic biota might be associated with other springs, the likelihood of actual exposure of aquatic biota is low because aquatic habitats associated with most springs are ephemeral in nature and provide limited year-round use. In contrast, Burgermeister Spring and its drainage support the largest amount of permanent aquatic habitat, including the uppermost portion of Lake 34, and thus have the greatest potential for exposure of aquatic biota. The use of contaminant concentrations reported from all springs together with the Burgermeister Spring exposure area should, therefore, fully cover the risks to aquatic biota associated with the springs of the chemical plant and ordnance works areas. An exception might be the springs within the lower segment of the Southeast Drainage (5300 drainage). Although the aquatic habitats immediately above and below the springs in the 5300 drainage are ephemeral, the lowermost portion of the drainage contains permanent year-round aquatic habitat with direct connection to the Missouri River. Higher concentrations of metals and nitroaromatic compounds have been detected in surface water and sediment from the Southeast.Drainage than from Burgermeister Spring or other area springs, and a separate ecological risk assessment has been conducted as part of an engineering evaluation/cost analysis for a proposed removal action at the drainage (DOE 1996).

Ingestion of contaminated drinking water is considered the principal exposure pathway for terrestrial biota in this study, and each spring in the ordnance works area represents a potential drinking water source. Risks were evaluated for selected terrestrial wildlife receptors (American robin and white-tailed deer). The exposure scenarios considered in this study consist only of contaminant uptake through ingestion of surface water; however, most of the springs are small and/or ephemeral in nature and, thus, individually are not likely to represent a significant portion of the drinking water supply for any wildlife receptor. Potential risks were calculated using the same surface water concentrations and exposure areas as were used for evaluating risks to aquatic biota. Burgermeister Spring and its immediate downstream waters likely exceed the total exposure area of all spring discharge points combined; therefore, the use of Burgermeister Spring as the exposure area in this exposure scenario should maximize the potential for contaminant uptake via ingestion of drinking water. 


\subsection{ESTIMATION OF EXPOSURE POINT CONCENTRATIONS}

A media-specific concentration of a contaminant at the location of exposure (i.e., exposure point concentration [EPC]) must be estimated to calculate the potential human and biota exposure that might be associated with a contaminated source or medium. For the human health component of this risk assessment, an EPC was determined for each COPC using the lower of the 95\% UCL of the arithmetic mean or the maximum value detected during the 1995 DOE/DA joint sampling rounds (see Section 2.2). The nature and extent of contamination defined by the data from the 1995 joint sampling rounds were comparable to the nature and extent of contamination defined by previously collected data (i.e., pre-1995). The EPCs are listed in Tables 3.2, 3.3, and 3.4 for the current and future recreational visitor and in Tables 3.5 through 3.9 for the hypothetical future resident. These concentrations were used to calculate hazard indices and risks for a recreational visitor who ingested or came into dermal contact with spring water from any of the springs. The results provide a range of potential human health impacts from these springs.

Groundwater calculations were performed for each well because the results from the monitoring networks covering the two areas indicated that contaminant concentrations are heterogeneous. A more detailed discussion of the nature and extent of contamination is presented in Chapter 4 of the RI report (DOE and DA 1997).

Future EPCs were assumed to be the same as current concentrations. This is a conservative assumption because attenuation of contaminant concentrations is anticipated over time as a result of removal of contaminant sources such as soil and raffinate pit sludge.

\subsection{ESTIMATION OF INTAKES}

Estimates of chemical and radioactive contaminant intakes were based on contaminant concentrations at the exposure points (Section 3.2) and on scenario-specific exposure assumptions and intake parameters. In accordance with EPA (1989b) guidance, the scenario-specific assumptions and intake parameters were based on the "reasonable maximum exposure" expected to occur for a given receptor under current and future land-use conditions. The recreational visitor was assumed to visit the area and drink water from a spring 20 times per year for 30 years. A water ingestion rate of $400 \mathrm{~mL}$ (about 2 cups) was assumed for each visit. For the hypothetical future resident calculations, it was assumed that the resident would drink $2 \mathrm{~L}$ ( 2.1 quarts) of water per day from a single well, 350 days per year, for 30 years. These and other assumptions are summarized in Table 3.1. For the current and future recreational visitor, calculations were performed for springs identified as representative of all springs in the area. The methodology used to calculate intakes and the results are presented in Section 3.3.1 for uranium and in Section 3.3.2 for the chemical contaminants. Cadmium, 1,3-DNB, nitrotoluenes, and nitrobenzene were identified in the RI as contaminants in the springs at levels greater than the statistically derived background values. However, because these contaminants were not detected in any samples collected during the 1995 joint DOE/DA sampling 
rounds, intakes were not calculated (these compounds were detected at very low frequencies and low concentrations in the pre-1995 data set [DOE and DA 1997]).

\subsubsection{Radiological Intakes}

Intakes for radioactive contaminants were calculated similarly to those for chemical carcinogens (see Section 3.3.2). Radiological intake is the amount of contaminant taken into the body per unit time, expressed in $\mathrm{pCi}$. The intake of radioactive contaminant $\mathrm{i}\left(\mathrm{I}_{\mathrm{i}}\right)$ from ingestion of groundwater or spring water was calculated as follows:

$$
I_{i}=R_{w i} \times I R \times E F \times E D
$$

where:

$$
\begin{aligned}
& R_{\mathrm{wi}}=\text { concentration of radionuclide in groundwater or spring water; } \\
& \mathrm{IR}=\text { ingestion rate; } \\
& \mathrm{EF}=\text { exposure frequency; and } \\
& \mathrm{ED}=\text { exposure duration. }
\end{aligned}
$$

The intake of radioactive contaminant $i\left(I_{i}\right)$ from dermal contact with spring water and groundwater was calculated as follows:

$$
I_{i}=R_{w i} \times S A \times P C_{i} \times C F \times E T \times E F \times E D
$$

where:

$$
\begin{aligned}
& \text { SA }=\text { surface area exposed }\left(\mathrm{cm}^{2}\right) ; \\
& \text { PC = permeability coefficient }(\mathrm{cm} / \mathrm{h}) ; \\
& \text { CF }=\text { conversion factor }\left(1 \times 10^{-3} \mathrm{~L} / \mathrm{cm}^{3}\right) ; \text { and } \\
& \text { ET }=\text { exposure time }(\mathrm{h}) .
\end{aligned}
$$

Estimated radiological intakes are provided in Tables 3.4 and 3.9 for the recreational visitor and residential scenarios, respectively. 


\subsubsection{Chemical Intakes}

Exposure to chemical contaminants is expressed in terms of intake. Intake is the amount of contaminant taken into the body per unit body weight per unit time (expressed as $\mathrm{mg} / \mathrm{kg}-\mathrm{d}$ ). The intakes of chemical contaminant $i\left(I_{i}\right)$ from ingestion of groundwater and spring water was calculated as follows:

$$
I_{i}=\frac{C_{w i} \times I R \times E F \times E D}{B W \times A T}
$$

where:

$$
\begin{aligned}
& \mathrm{C}_{\mathrm{wi}}=\text { concentration of contaminant in groundwater or spring water; } \\
& \mathrm{IR}=\text { ingestion rate; } \\
& \mathrm{EF}=\text { exposure frequency; } \\
& \mathrm{ED}=\text { exposure duration; } \\
& \mathrm{BW}=\text { average body weight over the exposure period }(\mathrm{kg}) ; \text { and } \\
& \mathrm{AT}=\text { averaging time (d). }
\end{aligned}
$$

The intake of chemical contaminant $i\left(\mathrm{I}_{\mathbf{i}}\right)$ from dermal contact with spring water and groundwater was calculated as follows:

$$
I_{i}=\frac{C_{w i} \times S A \times P C_{i} \times C F \times E T \times E F \times E D}{B W \times A T}
$$

where:

$$
\begin{aligned}
& \mathrm{C}_{\mathrm{wi}}=\text { concentration of contaminant in groundwater or spring water; } \\
& \mathrm{SA}=\text { skin surface area }\left(\mathrm{cm}^{2} / \text { event }\right) \\
& \mathrm{PCi}=\text { dermal permeability coefficient for contaminant } \mathrm{i}(\mathrm{cm} / \mathrm{h}) ; \text { and } \\
& \mathrm{CF}=\text { conversion factor }\left(1 \times 10^{-3} \mathrm{~L} / \mathrm{cm}^{3}\right) .
\end{aligned}
$$

Tables 3.2, 3.3, and 3.5 through 3.8 present the chemical exposure point concentrations and estimated intakes for the recreational and residential scenarios. 
The following equation was used to calculate intake of TCE from groundwater through inhalation while showering:

$$
I_{i}=\frac{C_{i} \times I R \times E T \times E F \times E D}{B W \times A F \times A D}
$$

where:

$$
\begin{aligned}
\mathrm{C}_{\mathrm{i}}= & \begin{array}{l}
\text { concentration of chemical } \mathrm{i}(\mathrm{TCE}) \text { in shower air }\left(\mathrm{mg} / \mathrm{m}^{3}\right) \text { - calculated } \\
\text { by multiplying the water exposure point concentration in } \mathrm{mg} / \mathrm{L}
\end{array} \\
& (\text { Table } 3.8) \text { times the water volume per shower }(200 \mathrm{~L}) \text { divided by a } \\
& \text { shower volume of } 2.5 \mathrm{~m}^{3} \text { and then dividing the total quantity by } 2 \text { (see } \\
& \text { DOE [1993] for methodology); } \\
\mathrm{IR} \mathrm{=} & \text { inhalation rate }\left(\mathrm{m}^{3} / \mathrm{h}\right) ; \\
\mathrm{ET}= & \text { exposure time }(\mathrm{h} / \mathrm{d}) ; \\
\mathrm{EF}= & \text { exposure frequency }(\mathrm{d} / \mathrm{yr}) ; \\
\mathrm{ED}= & \text { exposure duration }(\mathrm{yr}) ; \\
\mathrm{BW}= & \text { body weight }(\mathrm{kg}) ; \\
\mathrm{AF}= & \text { averaging frequency }(365 \mathrm{~d} / \mathrm{yr}) ; \text { and }
\end{aligned}
$$
by multiplying the water exposure point concentration in $\mathrm{mg} / \mathrm{L}$ (Table 3.8) times the water volume per shower $(200 \mathrm{~L})$ divided by a shower volume of $2.5 \mathrm{~m}^{3}$ and then dividing the total quantity by 2 (see

\subsection{ECOLOGICAL RESOURCES EXPOSURE AND EFFECTS ASSESSMENT}

\subsubsection{Methodology}

For aquatic biota, the risk assessment included consideration of both exposure and effects. Biotic surveys of the fish and invertebrate communities were conducted using the EPA Rapid Bioassessment Protocols (EPA 1989a). This method provided direct information on (1) the status of the aquatic community inhabiting Burgermeister Spring and exposed to the COECs and (2) the habitat quality of the spring and receiving drainage. Samples of surface water and sediment collected from Burgermeister Spring were tested for toxicity to evaluate potential effects of current levels of contamination in the spring to aquatic biota. Acute and chronic toxicity tests were conducted for two invertebrates (Daphnia and Hyalella), a fish (Pimephales), and an amphibian (Xenopus). These test organisms represent the major taxonomic categories of aquatic biota that occur in the spring and its 
downstream drainage. Fish and invertebrate samples were also collected from Burgermeister Spring for tissue analysis.

The risk assessment for terrestrial wildlife modeled uptake of each contaminant through the drinking water pathway for two receptor species, the white-tailed deer (Odocoileus virginianus) and the American robin (Turdus migratorius). The uptake modeling permitted prediction of an applied daily dose (ADD) for each receptor and each contaminant. Contaminant uptake from the ingestion of contaminated drinking water was estimated with the following equation:

$$
\mathrm{ADD}_{\mathrm{dw}}=\mathrm{C}_{\mathrm{dw}} \times \mathrm{FR} \times\left(\mathrm{IR}_{\mathrm{dw}} / \mathrm{BW}\right)
$$

where:

$$
\begin{aligned}
\mathrm{ADD}_{\mathrm{dw}}= & \text { applied daily dose from drinking water }(\mathrm{mg} / \mathrm{kg}-\mathrm{d}) ; \\
\mathrm{C}_{\mathrm{dw}}= & \text { exposure point concentration }(\mathrm{mg} / \mathrm{L}) \text { at the drinking water supply, } \\
& \text { using the maximum reported contaminant concentrations from all } \\
& \text { springs; }
\end{aligned}
$$

$\mathrm{FR}=$ fraction of total water ingested from contaminated source, using Burgermeister Spring as the drinking water supply;

$\mathrm{IR}_{\mathrm{dw}}=$ ingestion rate of drinking water $(\mathrm{g} / \mathrm{d})$; and

$\mathrm{BW}=$ body weight $(\mathrm{g})$ of the receptor.

Values of drinking water ingestion rates and body weights were obtained from the Wildlife Exposure Factors Handbook (EPA 1993) and the open scientific literature. The exposure factors used for this risk assessment are presented in Tables 3.10 and 3.11. Every effort was made to select exposure factors from populations nearest the August A. Busch Memorial Conservation Area. The fraction of total water ingestion by each receptor from Burgermeister Spring was estimated by centering the receptor home range on the spring, identifying all surface waters within the home range, and determining the percent contribution (by area) of the spring and its downstream waters (to Lake 34) to the total available surface water area within the receptor's home range. assumptions:

Modeling contaminant uptake and determining the ADD were based on the following

- Consistent with EPA (1993) guidance, the home range used in this assessment includes both daily activity and foraging ranges. 
- All foraging activities of each receptor are constant and uniformly distributed over the receptor's entire home range.

- Contaminant uptake by biota does not significantly affect the environmental concentration of contaminants.

- Contaminant assimilation is complete $(100 \%)$.

\subsubsection{Toxicity Results}

The results of the acute and chronic toxicity testing indicate some toxicity of surface water and sediment from Burgermeister Spring. Surface water toxicity, as indicated by reduced survival, was measured for two locations, SP-6301-1 and SP-6301-2 (Table 3.12). These locations correspond

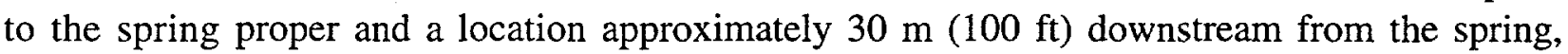
respectively. Toxicity at these locations was limited to the fish test biota; on the basis of either acute or chronic testing, no toxicity was evident for the other three test biota. Some surface water toxicity was also suggested for location SP-6301-3, which is downstream of the confluence of a large stream with the stream that originates at Burgermeister Spring. At SP-6301-3, no acute toxicity was indicated for any of the test biota, and chronic toxicity was observed only for Xenopus (30\% reduction in survival of exposed Xenopus; Table 3.12).

Sediment toxicity, as evidenced by reduced survival, was indicated for several locations (Table 3.13). Acute toxicity to Pimephales and chronic toxicity to Xenopus was indicated for sediment from location SP-6301-2. No acute toxicity was evident for any other test locations or biota. Toxicity to Hyalella was indicated for sediment collected directly from the spring (location SP-6301-1) and to Pimephales from the farthest downstream sampling location from the spring proper (SP-6301-4). Although survival was reduced in all of these tests, the survival rates were greater than $70 \%$ at all but the most downstream sampling location (Table 3.13).

\subsubsection{Tissue Analysis Results}

Macroinvertebrate and fish tissue samples were collected from Burgermeister Spring and analyzed for seven metals (arsenic, chromium, lead, mercury, selenium, silver, and uranium). The results of this analysis are presented in Appendix A of the RI report (DOE and DA 1997). Silver was not detected in either macroinvertebrate samples or fish samples, whereas mercury was detected only in fish samples and selenium only in macroinvertebrate samples. Estimated bioconcentration factors (BCFs) for macroinvertebrates (from sediment) and fish (from spring water) were typically less than 20; a BCF of 300 or more is considered to indicate significant bioconcentration (EPA 1989c). Only the BCF for mercury in fish exceeded a value of 300 , suggesting a potential for significant bioconcentration. 
Although the BCF for mercury in fish was high $(1,100)$, this value alone does not represent an effects concentration and does not indicate that fish in the Burgermeister Spring drainage are being impacted in any way. By definition, the BCF represents only the ratio between biological and environmental contaminant concentrations and is independent of effects. The measured tissue concentrations for fish from the Burgermeister Spring drainage are in the low end of the range of mercury tissue concentrations reported for freshwater fish in North America and within the wholebody concentration of $5,000 \mu \mathrm{g} / \mathrm{kg}$ suggested to be protective of freshwater fish by the U.S. Fish and Wildlife Service (Eisler 1987). In addition, the measured tissue concentrations in fish samples from Burgermeister Spring are not expected to pose a risk to piscivorous avian and mammalian wildlife. The measured concentrations are within the total mercury levels in prey suggested to be safe for birds $(100 \mu \mathrm{g} / \mathrm{kg})$ and small mammals $(1,100 \mu \mathrm{g} / \mathrm{kg})$ (Eisler 1987).

Thus, on the basis of the analysis of samples from Burgermeister Spring and the levels considered to be protective of fish and wildlife, the reported BCF values indicate that neither macroinvertebrates nor fish in Burgermeister Spring are accumulating contaminants from the environment at levels of concern.

\subsubsection{Biotic Survey Results}

Biotic surveys of the aquatic invertebrate and vertebrate biota inhabiting Burgermeister Spring indicate the presence of an aquatic community that would be expected to occur in similar spring systems and low-order headwater stream systems in the Midwest. No fish were collected from the spring proper, and the invertebrate community was dominated by amphipods and isopods (DOE and DA 1997). Fish are present in the drainage downstream of the spring proper. Although the fish community includes headwater stream fishes (e.g., orangethroat darter, brook silverside, and red shiner), it is dominated by juvenile fishes of species that typically inhabit slow-water streams and lakes (bluegill, green sunfish, largemouth bass, and black crappie), and it represents the strong influence of the fish community present in Lake 34 . These latter species become more abundant as one proceeds downstream from the spring to Lake 34. The absence of fish in the uppermost portion of Burgermeister Spring is due to the presence of a concrete weir across the stream; located about $15 \mathrm{~m}(50 \mathrm{ft})$ downstream of the spring, the weir serves as a barrier to the upstream passage of fish to the spring.

Habitat impairment and community quality were evaluated by following the EPA Rapid Bioassessment Protocols for fish and invertebrates (EPA 1989a). Burgermeister Spring and its downstream locations were found to support a limited fish community and slightly impaired aquatic invertebrate community (DOE and DA 1997), conditions that are probably a result of the physical characteristics of the spring rather than the contaminant levels. Flow in the uppermost segment of the stream is maintained almost exclusively by discharge at the spring; under low-flow conditions in the summer, the stream becomes intermittent and portions of the habitat become dry. The fish community at the lower end of the drainage is maintained by the permanent waters of Lake 34 . 
The amphibian survey results show that the amphibian community at Burgermeister Spring consists of species that are common to similar habitats throughout the Midwest and would be expected to inhabit the Burgermeister Spring drainage.

\subsubsection{Dose Estimates for Biota}

Contaminant uptake through ingestion of drinking water was estimated for the American robin and white-tailed deer using the uptake models presented in Section 3.4.1 and the exposure factors in Tables 3.8 and 3.9. For the American robin, $100 \%$ of the ingested drinking water was assumed to be obtained from Burgermeister Spring and downstream waters, whereas only $1.8 \%$ of the total water intake for the white-tailed deer was considered to come from this spring. These diet fractions were developed as the ratio of the total surface area of the Burgermeister Spring drainage (spring outflow to Lake 34 inflow) to the total available surface water area within the home range of each receptor (see Tables 3.10 and 3.11 for home range values). Contaminant uptake was modeled using the maximum contaminant concentrations reported from all springs.

Burgermeister Spring and its downstream waters was selected as the drinking water exposure point because the spring represents the largest and most permanent surface water body of all the springs. Although other springs in the area may be used by wildlife, most of these springs are very small and/or intermittent and, thus, probably do not represent a significant source of drinking water for terrestrial biota. Burgermeister Spring and its downstream waters likely exceed the total available surface water of all springs in the area, so use of the former as the drinking water exposure point maximizes the potential for contaminant uptake by the terrestrial receptors. Because maximum contaminant concentrations vary among the springs for any particular contaminant, the EPCs used in the uptake models were the maximum reported concentrations reported from all of the springs. Thus, the approach of using Burgermeister Spring as the drinking water exposure point area together with the maximum contaminant concentrations reported from any spring should result in very conservative estimates of contaminant uptake by terrestrial biota through ingestion of drinking water.

Modeling results are presented in Table 3.14. Uptake of nitroaromatic compounds through ingestion of drinking water was estimated to be very minor, with ingestion of any one compound being less than $0.001 \mathrm{mg} / \mathrm{kg}$-d for the white-tailed deer. Uptake of nitroaromatic compounds by the American robin was estimated to be less than $0.01 \mathrm{mg} / \mathrm{kg}$ - $\mathrm{d}$ for any one compound, except 2,4,6-TNT, which was estimated at $0.04 \mathrm{mg} / \mathrm{kg}$-d (Table 3.14). Similarly, the estimated daily uptake of metals was also typically very low for both receptor species. 
TABLE 3.1 Exposure Scenario Assumptions and Intake Parameters ${ }^{\mathrm{a}}$

\begin{tabular}{lccc}
\hline \multicolumn{1}{c}{ Parameter } & \multicolumn{3}{c}{ Current or Future } \\
Enit & Recreational Visitor & Future Resident \\
\hline Exposure time & h/event & 4 & $0.16^{\mathrm{b}}$ \\
Exposure frequency & events/yr & 20 & 350 \\
Exposure duration & $\mathrm{yr}$ & 30 & 30 \\
Body weight & $\mathrm{kg}$ & 70 & $70(4)^{\mathrm{c}}$ \\
Spring water ingestion rate & $\mathrm{mL} / \mathrm{event}$ & 400 & $\mathrm{NA}^{\mathrm{d}}$ \\
Groundwater ingestion rate & $\mathrm{L} / \mathrm{event}$ & $\mathrm{NA}$ & $2(0.64)^{\mathrm{c}}$ \\
Inhalation rate & $\mathrm{m}^{3} / \mathrm{h}$ & $\mathrm{NA}$ & 0.83 \\
$\quad$ (showering scenario for TCE only) & & & \\
Surface area & $\mathrm{cm} 2$ & $4,200^{\mathrm{e}}$ & $20,000^{\mathrm{f}}$ \\
Permeability coefficient & $\mathrm{cm} / \mathrm{h}$ & & $1 \times 10^{-3}$ \\
$\quad$ Default & & $1 \times 10^{-3}$ & $1.6 \times 10^{-2}$ \\
$\quad$ TCE & & $\mathrm{NA}$ &
\end{tabular}

a Assumptions and intake parameters are consistent with recommendations by the EPA (1995b, 1992a).

b Assumed length of time per day for showering.

c Exposure assumptions in parentheses are for an infant ingesting groundwater. These parameters were used to calculate intakes and hazard quotients for nitrates in groundwater because of the greater sensitivity of infants to the toxic effects of this contaminant.

d NA = not applicable.

e Surface area consists of the arms, hands, and lower legs (EPA 1992a).

f Surface area is the whole body (EPA 1992a). 
TABLE 3.2 Estimated Noncarcinogenic Intakes of COPCs for the Current and Future Recreational Visitor ${ }^{\mathrm{a}}$

\begin{tabular}{|c|c|c|c|c|c|c|c|c|c|c|c|c|}
\hline \multirow[b]{3}{*}{$\begin{array}{l}\text { Spring } \\
\text { ID }\end{array}$} & \multicolumn{3}{|c|}{ Antimony } & \multicolumn{3}{|c|}{ Iron } & \multicolumn{3}{|c|}{ Lithium } & \multicolumn{3}{|c|}{ Manganese } \\
\hline & \multirow[b]{2}{*}{$\begin{array}{l}\mathrm{EPC}^{\mathrm{b}} \\
(\mu \mathrm{g} / \mathrm{L})\end{array}$} & \multicolumn{2}{|c|}{ Intake (mg/kg-d) } & \multirow[b]{2}{*}{$\begin{array}{c}E^{E P C}{ }^{b} \\
(\mu \mathrm{g} / \mathrm{L})\end{array}$} & \multicolumn{2}{|c|}{ Intake (mg/kg-d) } & \multirow[b]{2}{*}{$\begin{array}{l}\mathrm{EPC}^{\mathrm{b}} \\
(\mu \mathrm{g} / \mathrm{L})\end{array}$} & \multicolumn{2}{|c|}{ Intake (mg/kg-d) } & \multirow[b]{2}{*}{$\begin{array}{l}\mathrm{EPC}^{\mathrm{b}} \\
(\mu \mathrm{g} / \mathrm{L})\end{array}$} & \multicolumn{2}{|c|}{ Intake $(\mathrm{mg} / \mathrm{kg}-\mathrm{d})$} \\
\hline & & Ingestion & Dermal & & Ingestion & Dermal & & Ingestion & Dermal & & Ingestion & Dermal \\
\hline 5101 & $2.7^{\mathrm{c}}$ & $4.2 \times 10^{-7}$ & $8.9 \times 10^{-9}$ & $790^{c}$ & $1.2 \times 10^{-4}$ & $2.6 \times 10^{-6}$ & $2.6^{\mathrm{c}}$ & $4.1 \times 10^{-7}$ & $8.5 \times 10^{-9}$ & $55^{\mathrm{c}}$ & $8.6 \times 10^{-6}$ & $1.8 \times 10^{-7}$ \\
\hline 5201 & 2.1 & $3.3 \times 10^{-7}$ & $6.9 \times 10^{-9}$ & 170 & $2.7 \times 10^{-5}$ & $5.7 \times 10^{-7}$ & $-{ }^{d}$ & - & - & 5.4 & $8.4 \times 10^{-7}$ & $1.8 \times 10^{-8}$ \\
\hline 5303 & 2.8 & $4.3 \times 10^{-7}$ & $9.1 \times 10^{-9}$ & 1,200 & $1.9 \times 10^{-4}$ & $4.0 \times 10^{-6}$ & 8.7 & $1.4 \times 10^{-6}$ & $2.9 \times 10^{-8}$ & 31 & $4.8 \times 10^{-6}$ & $1.0 \times 10^{-7}$ \\
\hline 5402 & $1.9^{c}$ & $3.0 \times 10^{-7}$ & $6.2 \times 10^{-9}$ & 760 & $1.2 \times 10^{-4}$ & $2.5 \times 10^{-6}$ & - & - & - & 9.8 & $1.5 \times 10^{-6}$ & $3.2 \times 10^{-8}$ \\
\hline 5501 & $2.6^{\mathrm{c}}$ & $4.1 \times 10^{-7}$ & $8.5 \times 10^{-9}$ & $280^{\mathrm{c}}$ & $4.4 \times 10^{-5}$ & $9.2 \times 10^{-7}$ & $2.3^{\mathrm{c}}$ & $3.6 \times 10^{-7}$ & $7.6 \times 10^{-9}$ & 30 & $4.6 \times 10^{-6}$ & $9.7 \times 10^{-8}$ \\
\hline 5504 & 1.4 & $2.2 \times 10^{-7}$ & $4.7 \times 10^{-9}$ & $140^{\mathrm{c}}$ & $2.1 \times 10^{-5}$ & $4.4 \times 10^{-7}$ & - & - & - & 5.8 & $9.1 \times 10^{-7}$ & $1.9 \times 10^{-8}$ \\
\hline 5601 & 3.4 & $5.3 \times 10^{-7}$ & $1.1 \times 10^{-8}$ & 1,000 & $1.6 \times 10^{-4}$ & $3.4 \times 10^{-6}$ & - & - & - & 220 & $3.4 \times 10^{-5}$ & $7.2 \times 10^{-7}$ \\
\hline 5602 & 8 & $1.3 \times 10^{-6}$ & $2.6 \times 10^{-8}$ & 500 & $7.8 \times 10^{-5}$ & $1.6 \times 10^{-6}$ & - & - & - & 210 & $3.3 \times 10^{-5}$ & $7.0 \times 10^{-7}$ \\
\hline 5605 & $2.7^{\mathrm{c}}$ & $4.2 \times 10^{-7}$ & $8.9 \times 10^{-9}$ & $360^{\mathrm{c}}$ & $5.6 \times 10^{-5}$ & $1.2 \times 10^{-6}$ & - & - & - & $32^{\mathrm{c}}$ & $5.0 \times 10^{-6}$ & $1.1 \times 10^{-7}$ \\
\hline 5612 & $13^{c}$ & $2.0 \times 10^{-6}$ & $4.3 \times 10^{-8}$ & $86^{c}$ & $1.4 \times 10^{-5}$ & $2.8 \times 10^{-7}$ & - & - & - & $9.6^{c}$ & $1.5 \times 10^{-6}$ & $3.2 \times 10^{-8}$ \\
\hline 6301 & $2.1^{\mathrm{c}}$ & $3.3 \times 10^{-7}$ & $6.9 \times 10^{-9}$ & 390 & $6.0 \times 10^{-5}$ & $1.3 \times 10^{-6}$ & $18^{\mathrm{c}}$ & $2.8 \times 10^{-6}$ & $6.0 \times 10^{-8}$ & 18 & $2.9 \times 10^{-6}$ & $6.0 \times 10^{-8}$ \\
\hline 6303 & 5.5 & $8.6 \times 10^{-7}$ & $1.8 \times 10^{-8}$ & 980 & $1.5 \times 10^{-4}$ & $3.2 \times 10^{-6}$ & $3.6^{\mathrm{c}}$ & $5.6 \times 10^{-7}$ & $1.2 \times 10^{-8}$ & $52^{c}$ & $8.1 \times 10^{-6}$ & $1.7 \times 10^{-7}$ \\
\hline 6306 & 2.3 & $3.7 \times 10^{-7}$ & $7.7 \times 10^{-9}$ & $7,300^{\mathrm{c}}$ & $1.1 \times 10^{-3}$ & $2.4 \times 10^{-5}$ & - & - & - & $8,600^{c}$ & $1.3 \times 10^{-3}$ & $2.8 \times 10^{-5}$ \\
\hline 6501 & $1.4^{\mathrm{c}}$ & $2.2 \times 10^{-7}$ & $4.6 \times 10^{-9}$ & $650^{\mathrm{c}}$ & $1.0 \times 10^{-4}$ & $2.1 \times 10^{-6}$ & - & - & - & 29 & $4.6 \times 10^{-6}$ & $9.6 \times 10^{-8}$ \\
\hline 6601 & 4.8 & $7.6 \times 10^{-7}$ & $1.6 \times 10^{-8}$ & 340 & $5.3 \times 10^{-5}$ & $1.1 \times 10^{-6}$ & - & - & - & $45^{\mathrm{c}}$ & $7.1 \times 10^{-6}$ & $1.5 \times 10^{-7}$ \\
\hline
\end{tabular}


TABLE 3.2 (Cont.)

\begin{tabular}{|c|c|c|c|c|c|c|c|c|c|c|c|c|}
\hline \multirow[b]{3}{*}{$\begin{array}{l}\text { Spring } \\
\text { ID }\end{array}$} & \multirow[b]{3}{*}{$\begin{array}{c}\mathrm{EPC}^{\mathrm{b}} \\
(\mu \mathrm{g} / \mathrm{L})\end{array}$} & \multicolumn{2}{|c|}{ Mercury } & \multicolumn{3}{|c|}{ Molybdenum } & \multicolumn{3}{|c|}{ Silver } & \multicolumn{3}{|c|}{ Uranium, Total } \\
\hline & & \multicolumn{2}{|c|}{ Intake (mg/kg-d) } & \multirow[b]{2}{*}{$\begin{array}{c}\mathrm{EPC}^{\mathrm{b}} \\
(\mu \mathrm{g} / \mathrm{L}) \\
\end{array}$} & \multicolumn{2}{|c|}{ Intakc (mg/kg-d) } & \multirow[b]{2}{*}{$\begin{array}{c}\mathrm{EPC}^{\mathrm{b}} \\
(\mu \mathrm{g} / \mathrm{L})\end{array}$} & \multicolumn{2}{|c|}{ Intake (mg/kg-d) } & \multirow[b]{2}{*}{$\begin{array}{c}\mathrm{EPC}^{\mathrm{b}} \\
(\mu \mathrm{g} / \mathrm{L})\end{array}$} & \multicolumn{2}{|c|}{ Intake (mg/kg-d) } \\
\hline & & Ingestion & Dermal & & Ingestion & Dermal & & Ingestion & Dermal & & Ingestion & Dermal \\
\hline 5101 & - & - & - & - & - & - & - & - & - & $0.57^{\mathrm{c}}$ & $9.0 \times 10^{-8}$ & $1.9 \times 10^{-9}$ \\
\hline 5201 & - & - & - & - & - & - & - & - & - & $1.3^{\mathrm{c}}$ & $2.0 \times 10^{-7}$ & $4.2 \times 10^{-9}$ \\
\hline 5303 & 0.94 & $1.5 \times 10^{-7}$ & $3.1 \times 10^{-9}$ & 13 & $2.1 \times 10^{-6}$ & $4.4 \times 10^{-8}$ & - & - & - & $180^{c}$ & $2.9 \times 10^{-5}$ & $6.0 \times 10^{-7}$ \\
\hline 5402 & - & - & - & - & - & - & - & - & - & $1.4^{\mathrm{c}}$ & $2.2 \times 10^{-7}$ & $4.7 \times 10^{-9}$ \\
\hline 5501 & - & - & - & - & - & - & - & - & - & $1.1^{\mathrm{c}}$ & $1.7 \times 10^{-7}$ & $3.6 \times 10^{-9}$ \\
\hline 5504 & - - & - & - & - & - & - & 1.5 & $2.4 \times 10^{-7}$ & $5.0 \times 10^{-9}$ & $0.75^{\mathfrak{c}}$ & $1.2 \times 10^{-7}$ & $2.5 \times 10^{-9}$ \\
\hline 5601 & - & - & - & - & - & - & - & - & - & $0.67^{c}$ & $1.0 \times 10^{-7}$ & $2.2 \times 10^{-9}$ \\
\hline 5602 & - & - & - & - & - & - & - & - & - & $0.5^{\mathrm{c}}$ & $7.8 \times 10^{-8}$ & $1.6 \times 10^{-9}$ \\
\hline 5605 & - & - & - & - & - & - & - & - & - & $0.93^{c}$ & $1.4 \times 10^{-7}$ & $3.0 \times 10^{-9}$ \\
\hline 5612 & - & - & - & - & - & - & - & - & - & $0.77^{\mathfrak{c}}$ & $1.2 \times 10^{-7}$ & $2.5 \times 10^{-9}$ \\
\hline 6301 & - & - & - & - & - & - & 2 & $3.1 \times 10^{-7}$ & $6.6 \times 10^{-9}$ & 95 & $1.5 \times 10^{-5}$ & $3.1 \times 10^{-7}$ \\
\hline 6303 & - & - & - & - & - & - & - & - & - & $1.9^{c}$ & $3.0 \times 10^{-7}$ & $6.2 \times 10^{-9}$ \\
\hline 6306 & - & - & - & $4.7^{\mathrm{c}}$ & $7.4 \times 10^{-7}$ & $1.5 \times 10^{-8}$ & - & - & - & $1^{c}$ & $1.6 \times 10^{-7}$ & $3.4 \times 10^{-9}$ \\
\hline 6501 & - & - & - & - & - & - & 31 & $4.8 \times 10^{-6}$ & $1.0 \times 10^{-7}$ & 3.6 & $5.6 \times 10^{-7}$ & $1.2 \times 10^{-8}$ \\
\hline 6601 & - & - & - & - & - & - & - & - & - & $0.56^{\mathrm{c}}$ & $8.8 \times 10^{-8}$ & $1.9 \times 10^{-9}$ \\
\hline
\end{tabular}


TABLE 3.2 (Cont.)

\begin{tabular}{|c|c|c|c|c|c|c|c|c|c|}
\hline \multirow[b]{3}{*}{$\begin{array}{c}\text { Spring } \\
\text { ID }\end{array}$} & \multicolumn{3}{|c|}{ Chloride } & \multicolumn{3}{|c|}{ Nitrate-N } & \multicolumn{3}{|c|}{ Sulfate } \\
\hline & \multirow[b]{2}{*}{$\begin{array}{c}\mathrm{EPC}^{\mathrm{b}} \\
(\mu \mathrm{g} / \mathrm{L})\end{array}$} & \multicolumn{2}{|c|}{ Intake (mg/kg-d) } & \multirow[b]{2}{*}{$\begin{array}{c}\mathrm{EPC}^{\mathrm{b}} \\
(\mu \mathrm{g} / \mathrm{L})\end{array}$} & \multicolumn{2}{|c|}{ Intake (mg/kg-d) } & \multirow[b]{2}{*}{$\begin{array}{l}\mathrm{EPC}^{\mathrm{b}} \\
(\mu \mathrm{g} / \mathrm{L})\end{array}$} & \multicolumn{2}{|c|}{ Intake (mg/kg-d) } \\
\hline & & Ingestion & Dermal & & Ingestion & Dermal & & Ingestion & Dermal \\
\hline 5101 & 35,000 & $5.5 \times 10^{-3}$ & $1.2 \times 10^{-4}$ & $2,500^{\mathrm{c}}$ & $3.9 \times 10^{-4}$ & $8.2 \times 10^{-6}$ & 48,000 & $7.5 \times 10^{-3}$ & $1.6 \times 10^{-4}$ \\
\hline 5201 & 13,000 & $2.0 \times 10^{-3}$ & $4.3 \times 10^{-5}$ & $170^{\mathrm{c}}$ & $2.7 \times 10^{-5}$ & $5.6 \times 10^{-7}$ & 36,000 & $5.6 \times 10^{-3}$ & $1.2 \times 10^{-4}$ \\
\hline 5303 & 21,000 & $3.3 \times 10^{-3}$ & $6.9 \times 10^{-5}$ & $2,100^{\mathrm{c}}$ & $3.3 \times 10^{-4}$ & $6.9 \times 10^{-6}$ & 51,000 & $8.0 \times 10^{-3}$ & $1.7 \times 10^{-4}$ \\
\hline 5402 & 22,000 & $3.4 \times 10^{-3}$ & $7.2 \times 10^{-5}$ & $420^{\mathrm{c}}$ & $6.5 \times 10^{-5}$ & $1.4 \times 10^{-6}$ & 23,000 & $3.6 \times 10^{-3}$ & $7.6 \times 10^{-5}$ \\
\hline 5501 & 5,400 & $8.5 \times 10^{-4}$ & $1.8 \times 10^{-5}$ & $410^{\mathrm{c}}$ & $6.4 \times 10^{-5}$ & $1.3 \times 10^{-6}$ & 39,000 & $6.1 \times 10^{-3}$ & $1.3 \times 10^{-4}$ \\
\hline 5504 & 2,700 & $4.2 \times 10^{-4}$ & $8.9 \times 10^{-6}$ & - & - & - & 30,000 & $4.7 \times 10^{-3}$ & $9.9 \times 10^{-5}$ \\
\hline 5601 & 2,100 & $3.3 \times 10^{-4}$ & $6.9 \times 10^{-6}$ & - & - & - & 18,000 & $2.8 \times 10^{-3}$ & $5.9 \times 10^{-5}$ \\
\hline 5602 & 1,100 & $1.7 \times 10^{-4}$ & $3.6 \times 10^{-6}$ & $460^{\mathrm{c}}$ & $7.2 \times 10^{-5}$ & $1.5 \times 10^{-6}$ & 14,000 & $2.2 \times 10^{-3}$ & $4.6 \times 10^{-5}$ \\
\hline 5605 & 2,600 & $4.1 \times 10^{-4}$ & $8.5 \times 10^{-6}$ & $140^{\mathrm{c}}$ & $2.2 \times 10^{-5}$ & $4.6 \times 10^{-7}$ & 21,000 & $3.3 \times 10^{-3}$ & $6.9 \times 10^{-5}$ \\
\hline 5612 & 2,200 & $3.4 \times 10^{-4}$ & $7.2 \times 10^{-6}$ & - & - & - & 23,000 & $3.6 \times 10^{-3}$ & $7.6 \times 10^{-5}$ \\
\hline 6301 & 14,000 & $2.2 \times 10^{-3}$ & $4.6 \times 10^{-5}$ & 18,000 & $2.7 \times 10^{-3}$ & $5.8 \times 10^{-5}$ & 43,000 & $6.7 \times 10^{-3}$ & $1.4 \times 10^{-4}$ \\
\hline 6303 & 3,300 & $5.2 \times 10^{-4}$ & $1.1 \times 10^{-5}$ & $12,000^{\mathrm{c}}$ & $1.9 \times 10^{-3}$ & $3.9 \times 10^{-5}$ & 25,000 & $3.9 \times 10^{-3}$ & $8.2 \times 10^{-5}$ \\
\hline 6306 & 7,500 & $1.2 \times 10^{-3}$ & $2.5 \times 10^{-5}$ & - & - & - & - & - & - \\
\hline 6501 & 2,400 & $3.8 \times 10^{-4}$ & $7.9 \times 10^{-6}$ & 450 & $7.1 \times 10^{-5}$ & $1.5 \times 10^{-6}$ & 15,000 & $2.3 \times 10^{-3}$ & $4.9 \times 10^{-5}$ \\
\hline 6601 & 2,100 & $3.3 \times 10^{-4}$ & $6.9 \times 10^{-6}$ & $760^{c}$ & $1.2 \times 10^{-4}$ & $2.5 \times 10^{-6}$ & 14,000 & $2.2 \times 10^{-3}$ & $4.6 \times 10^{-5}$ \\
\hline
\end{tabular}


TABLE 3.2 (Cont.)

\begin{tabular}{|c|c|c|c|c|c|c|c|c|c|}
\hline \multirow[b]{3}{*}{$\begin{array}{l}\text { Spring } \\
\text { ID }\end{array}$} & \multirow[b]{3}{*}{$\begin{array}{c}\mathrm{EPC}^{b} \\
(\mu \mathrm{g} / \mathrm{L})\end{array}$} & \multicolumn{2}{|c|}{ 1,3,5-TNB } & \multicolumn{3}{|c|}{$2,4,6-\mathrm{TNT}$} & \multicolumn{3}{|c|}{ 2,4-DNT } \\
\hline & & \multicolumn{2}{|c|}{ Intake (mg/kg-d) } & \multirow[b]{2}{*}{$\begin{array}{c}\mathrm{EPC}^{\mathrm{b}} \\
(\mu \mathrm{g} / \mathrm{L})\end{array}$} & \multicolumn{2}{|c|}{ Intake (mg/kg-d) } & \multirow[b]{2}{*}{$\begin{array}{c}\mathrm{EPC}^{\mathbf{b}} \\
(\mu \mathrm{g} / \mathrm{L})\end{array}$} & \multicolumn{2}{|c|}{ Intake (mg/kg-d) } \\
\hline & & Ingestion & Dermal & & Ingestion & Dermal & & Ingestion & Dermal \\
\hline 5101 & - & - & - & - & - & - & - & - & - \\
\hline 5201 & $6^{\mathrm{c}}$ & $9.4 \times 10^{-7}$ & $2.0 \times 10^{-8}$ & $110^{\mathrm{c}}$ & $1.7 \times 10^{-5}$ & $3.6 \times 10^{-7}$ & $0.076^{\mathrm{c}}$ & $1.2 \times 10^{-8}$ & $2.5 \times 10^{-10}$ \\
\hline 5303 & $0.41^{\mathrm{c}}$ & $6.4 \times 10^{-8}$ & $1.3 \times 10^{-9}$ & $120^{\mathrm{c}}$ & $1.9 \times 10^{-5}$ & $3.9 \times 10^{-7}$ & $0.21^{\mathrm{c}}$ & $3.3 \times 10^{-8}$ & $6.9 \times 10^{-10}$ \\
\hline 5402 & - & - & - & - & - & - & - & - & - \\
\hline 5501 & - & - & - & - & - & - & $0.046^{\mathrm{c}}$ & $7.2 \times 10^{-9}$ & $1.5 \times 10^{-10}$ \\
\hline 5504 & - & - & - & - & - & - & - & - & - \\
\hline 5601 & - & - & - & - & - & - & $0.04^{\mathfrak{c}}$ & $6.3 \times 10^{-9}$ & $1.3 \times 10^{-10}$ \\
\hline 5602 & $0.25^{\mathrm{c}}$ & $3.9 \times 10^{-8}$ & $8.2 \times 10^{-10}$ & $1^{c}$ & $1.6 \times 10^{-7}$ & $3.3 \times 10^{-9}$ & $0.13^{\mathrm{c}}$ & $2.0 \times 10^{-8}$ & $4.3 \times 10^{-10}$ \\
\hline 5605 & $0.096^{\mathrm{c}}$ & $1.5 \times 10^{-8}$ & $3.2 \times 10^{-10}$ & $4.8^{\mathrm{c}}$ & $7.5 \times 10^{-7}$ & $1.6 \times 10^{-8}$ & $0.15^{\mathfrak{c}}$ & $2.3 \times 10^{-8}$ & $4.9 \times 10^{-10}$ \\
\hline 5612 & - & - & - & $0.073^{\mathrm{c}}$ & $1.1 \times 10^{-8}$ & $2.4 \times 10^{-10}$ & - & - & - \\
\hline 6301 & 0.024 & $3.7 \times 10^{-9}$ & $7.7 \times 10^{-11}$ & 0.25 & $3.9 \times 10^{-8}$ & $8.2 \times 10^{-10}$ & 0.065 & $1.0 \times 10^{-8}$ & $2.1 \times 10^{-10}$ \\
\hline 6303 & $0.09^{c}$ & $1.4 \times 10^{-8}$ & $3.0 \times 10^{-10}$ & $1.5^{\mathrm{c}}$ & $2.3 \times 10^{-7}$ & $4.9 \times 10^{-9}$ & $0.15^{\mathrm{c}}$ & $2.3 \times 10^{-8}$ & $4.9 \times 10^{-10}$ \\
\hline 6306 & - & - & - & - & - & - & - & - & - \\
\hline 6501 & - & - & - & - & - & - & - & - & - \\
\hline 6601 & - & - & - & $0.02^{\mathrm{c}}$ & $3.1 \times 10^{-9}$ & $6.6 \times 10^{-11}$ & - & - & - \\
\hline
\end{tabular}


TABLE 3.2 (Cont.)

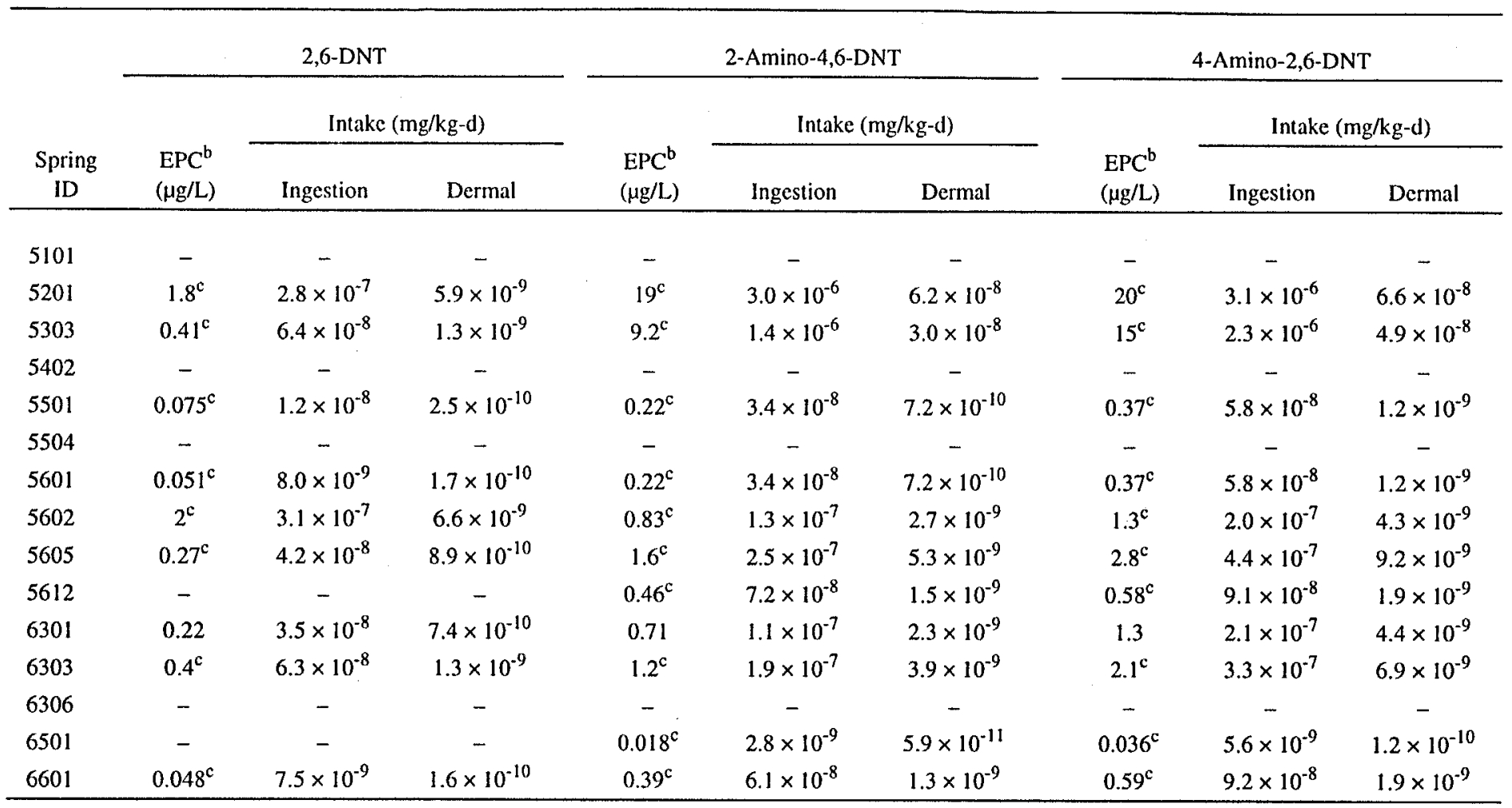

a Cadmium, 1,3-DNB, nitrobenzene, and nitrotoluenes were not detected in the 1995 joint DOE/DA sampling rounds.

b $\mathrm{EPC}=$ exposure point concentrations represented by the $95 \%$ UCL or the maximum concentration (indicated by footnote c).

c Value is the maximum concentration.

d A hyphen (-) indicates that the parameter was not detected. 
TABLE 3.3 Estimated Carcinogenic Intakes of COPCs for the Current and Future Recreational Visitor

\begin{tabular}{|c|c|c|c|c|c|c|c|c|c|}
\hline \multirow[b]{3}{*}{$\begin{array}{c}\text { Spring } \\
\text { ID }\end{array}$} & \multirow[b]{3}{*}{$\begin{array}{c}\mathrm{EPC}^{\mathrm{a}} \\
(\mu \mathrm{g} / \mathrm{L})\end{array}$} & \multicolumn{2}{|c|}{ 2,4,6-TNT } & \multicolumn{3}{|c|}{ 2,4,-DNT } & \multicolumn{3}{|c|}{ 2,6-DNT } \\
\hline & & \multicolumn{2}{|c|}{ Intake (mg/kg-d) } & \multirow[b]{2}{*}{$\begin{array}{c}\mathrm{EPC}^{\mathrm{a}} \\
(\mu \mathrm{g} / \mathrm{L})\end{array}$} & \multicolumn{2}{|c|}{ Intake $(\mathrm{mg} / \mathrm{kg}-\mathrm{d})$} & \multirow[b]{2}{*}{$\begin{array}{l}\mathrm{EPC}^{\mathrm{a}} \\
(\mu \mathrm{g} / \mathrm{L}) \\
\end{array}$} & \multicolumn{2}{|c|}{ Intake $(\mathrm{mg} / \mathrm{kg}-\mathrm{d})$} \\
\hline & & Ingestion & Dermal & & Ingestion & Dermal & & Ingestion & Dermal \\
\hline 5101 & $-b$ & - & - & - & - & - & - & - & - \\
\hline 5102 & - & - & - & - & - & - & - & - & - \\
\hline 5201 & $110^{\mathrm{c}}$ & $7.4 \times 10^{-6}$ & $1.5 \times 10^{-7}$ & 0.076 & $5.1 \times 10^{-9}$ & $1.1 \times 10^{-10}$ & 1.8 & $1.2 \times 10^{-7}$ & $2.5 \times 10^{-9}$ \\
\hline 5303 & 120 & $8.1 \times 10^{-6}$ & $1.7 \times 10^{-7}$ & 0.21 & $1.4 \times 10^{-8}$ & $3.0 \times 10^{-10}$ & 0.41 & $2.8 \times 10^{-8}$ & $5.8 \times 10^{-10}$ \\
\hline 5402 & - & - & - & - & - & - & - & - & - \\
\hline 5501 & - & - & - & 0.046 & $3.1 \times 10^{-9}$ & $6.5 \times 10^{-11}$ & 0.075 & $5.0 \times 10^{-9}$ & $1.1 \times 10^{-10}$ \\
\hline 5504 & - & - & - & - & - & - & - & - & - \\
\hline 5601 & - & - & - & 0.04 & $2.7 \times 10^{-9}$ & $5.6 \times 10^{-11}$ & 0.051 & $3.4 \times 10^{-9}$ & $7.2 \times 10^{-11}$ \\
\hline 5602 & 1 & $6.7 \times 10^{-8}$ & $1.4 \times 10^{-9}$ & 0.13 & $8.7 \times 10^{-9}$ & $1.8 \times 10^{-10}$ & 2 & $1.3 \times 10^{-7}$ & $2.8 \times 10^{-9}$ \\
\hline 5605 & 4.8 & $3.2 \times 10^{-7}$ & $6.8 \times 10^{-9}$ & 0.15 & $1.0 \times 10^{-8}$ & $2.1 \times 10^{-10}$ & 0.27 & $1.8 \times 10^{-8}$ & $3.8 \times 10^{-10}$ \\
\hline 5612 & 0.073 & $4.9 \times 10^{-9}$ & ${ }_{0}^{1.0} \times 10^{-1}$ & - & - & - & - & - & - \\
\hline 6301 & 0.25 & $1.7 \times 10^{-8}$ & ${ }_{0}^{3.5} \times 10^{-1}$ & 0.065 & $4.3 \times 10^{-9}$ & $9.1 \times 10^{-11}$ & 0.22 & $1.5 \times 10^{-8}$ & $3.2 \times 10^{-10}$ \\
\hline 6303 & 1.5 & $1.0 \times 10^{-7}$ & $2.1 \times 10^{-9}$ & 0.15 & $1.0 \times 10^{-8}$ & $2.1 \times 10^{-10}$ & 0.4 & $2.7 \times 10^{-8}$ & $5.6 \times 10^{-10}$ \\
\hline 6306 & - & - & - & - & - & - & - & - & - \\
\hline 6501 & - & - & - & - & - & - & - & - & - \\
\hline 6601 & 0.02 & $1.3 \times 10^{-9}$ & $2.8 \times 10^{-1}$ & - & - & - & 0.048 & $3.2 \times 10^{-9}$ & $6.8 \times 10^{-11}$ \\
\hline
\end{tabular}

a EPC = exposure point concentrations represented by the $95 \%$ UCL or the maximum concentration (indicated by footnote c).

b A hyphen (-) indicates that the parameter was not detected.

c Value is the maximum concentration. 
TABLE 3.4 Estimated Intakes of Uranium for the Current and Future Recreational Visitor

\begin{tabular}{|c|c|c|c|}
\hline \multirow[b]{3}{*}{ Spring ID } & \multicolumn{3}{|c|}{ Uranium } \\
\hline & \multirow{2}{*}{$\begin{array}{c}\mathrm{EPC}^{\mathrm{a}} \\
(\mathrm{pCi} / \mathrm{L})\end{array}$} & \multicolumn{2}{|c|}{ Intake (pCi) } \\
\hline & & Ingestion & Dermal \\
\hline 5101 & 0.38 & $9.1 \times 10^{1}$ & $9.6 \times 10^{-1}$ \\
\hline 5201 & 0.87 & $2.1 \times 10^{2}$ & 2.2 \\
\hline 5303 & 120 & $2.9 \times 10^{4}$ & $3.0 \times 10^{2}$ \\
\hline 5402 & 0.95 & $2.3 \times 10^{2}$ & 2.4 \\
\hline 5501 & 0.74 & $1.8 \times 10^{2}$ & 1.9 \\
\hline 5504 & 0.50 & $1.2 \times 10^{2}$ & 1.3 \\
\hline 5601 & 0.45 & $1.1 \times 10^{2}$ & 1.1 \\
\hline 5602 & 0.33 & $7.9 \times 10^{1}$ & $8.3 \times 10^{-1}$ \\
\hline 5605 & 0.62 & $1.5 \times 10^{2}$ & 1.6 \\
\hline 5612 & 0.52 & $1.2 \times 10^{2}$ & 1.3 \\
\hline 6301 & 91 & $2.2 \times 10^{4}$ & $2.3 \times 10^{2}$ \\
\hline 6303 & 1.3 & $3.1 \times 10^{2}$ & 3.3 \\
\hline 6306 & 0.69 & $1.7 \times 10^{2}$ & 1.7 \\
\hline 6501 & 2.8 & $6.7 \times 10^{2}$ & 7.1 \\
\hline 6601 & 0.38 & $9.1 \times 10^{1}$ & $9.6 \times 10^{-1}$ \\
\hline
\end{tabular}

${ }^{a}$ EPC $=$ exposure point concentration, which is the maximum uranium value for each spring from the 1995 joint DOE/DA sampling rounds. 
TABLE 3.5 Estimated Noncarcinogenic Intakes of Metal COPCs for the Hypothetical Future Resident

\begin{tabular}{|c|c|c|c|c|c|c|c|c|c|}
\hline \multirow[b]{3}{*}{ Well ID } & \multicolumn{3}{|c|}{ Lithium } & \multicolumn{3}{|c|}{ Molybdenum } & \multicolumn{3}{|c|}{ Uranium, Total } \\
\hline & \multirow{2}{*}{$\begin{array}{c}\mathrm{EPC}^{\mathrm{a}} \\
(\mu \mathrm{g} / \mathrm{L})\end{array}$} & \multicolumn{2}{|c|}{ Intake $(\mathrm{mg} / \mathrm{kg}-\mathrm{d})$} & \multirow{2}{*}{$\begin{array}{l}\mathrm{EPC}^{\mathrm{a}} \\
(\mu \mathrm{g} / \mathrm{L})\end{array}$} & \multicolumn{2}{|c|}{ Intake (mg/kg-d) } & \multirow{2}{*}{$\begin{array}{l}\mathrm{EPC}^{\mathrm{a}} \\
(\mu \mathrm{g} / \mathrm{L})\end{array}$} & \multicolumn{2}{|c|}{ Intake $(\mathrm{mg} / \mathrm{kg}-\mathrm{d})$} \\
\hline & & Ingestion & Dermal & & Ingestion & Dermal & & Ingestion & Dermal \\
\hline \multicolumn{10}{|l|}{ Deep Wells } \\
\hline MWD-05 & 20 & $5.4 \times 10^{-4}$ & $8.7 \times 10^{-7}$ & 1.3 & $3.6 \times 10^{-5}$ & $5.7 \times 10^{-8}$ & 0.86 & $2.4 \times 10^{-5}$ & $3.8 \times 10^{-8}$ \\
\hline MWD-18 & 6.7 & $1.8 \times 10^{-4}$ & $2.9 \times 10^{-7}$ & $-{ }^{b}$ & - & - & 1.2 & $3.2 \times 10^{-5}$ & $5.1 \times 10^{-8}$ \\
\hline MWGS-01 & - & - & - & - & - & - & - & - & - \\
\hline MWGS-02 & - & - & - & - & - & - & - & - & - \\
\hline MWS-18 & 9.4 & $2.6 \times 10^{-4}$ & $4.1 \times 10^{-7}$ & 7.6 & $2.1 \times 10^{-4}$ & $3.3 \times 10^{-7}$ & 2.3 & $6.3 \times 10^{-5}$ & $1.0 \times 10^{-7}$ \\
\hline MWS-101 & 1.1 & $3.0 \times 10^{-5}$ & $4.8 \times 10^{-8}$ & - & - & - & 0.80 & $2.2 \times 10^{-5}$ & $3.5 \times 10^{-8}$ \\
\hline MWS-102 & 7.4 & $2.0 \times 10^{-4}$ & $3.2 \times 10^{-7}$ & 6.7 & $1.8 \times 10^{-4}$ & $2.9 \times 10^{-7}$ & 3.9 & $1.1 \times 10^{-4}$ & $1.7 \times 10^{-7}$ \\
\hline MWS-103 & 4.2 & $1.2 \times 10^{-4}$ & $1.8 \times 10^{-7}$ & - & - & - & 1.1 & $3.1 \times 10^{-5}$ & $5.0 \times 10^{-8}$ \\
\hline TIL-3 & 3.7 & $1.0 \times 10^{-4}$ & $1.6 \times 10^{-7}$ & 2.5 & $6.8 \times 10^{-5}$ & $1.1 \times 10^{-7}$ & 0.16 & $4.4 \times 10^{-6}$ & $7.0 \times 10^{-9}$ \\
\hline \multicolumn{10}{|l|}{ Overburden } \\
\hline MW-2031 & - & - & - & - & - & - & - & - & - \\
\hline MW-2032 & 14 & $3.8 \times 10^{-4}$ & $6.1 \times 10^{-7}$ & 1.8 & $4.9 \times 10^{-5}$ & $7.9 \times 10^{-8}$ & 6.3 & $1.7 \times 10^{-4}$ & $2.7 \times 10^{-7}$ \\
\hline MW-2033 & 3.7 & $1.0 \times 10^{-4}$ & $1.6 \times 10^{-7}$ & - & - & - & 3.5 & $9.6 \times 10^{-5}$ & $1.5 \times 10^{-7}$ \\
\hline MW-3001 & - & - & - & - & - & - & - & - & - \\
\hline MW-3013 & - & - & - & - & - & - & - & - & - \\
\hline$M W-3018$ & - & - & - & - & - & - & - & - & - \\
\hline MW-3022 & - & - & - & - & - & - & - & - & - \\
\hline USGS-2A & - & - & - & - & - & - & - & - & - \\
\hline MWV-01 & 2.3 & $6.3 \times 10^{-5}$ & $1.0 \times 10^{-7}$ & - & - & - & 6.2 & $1.7 \times 10^{-4}$ & $2.7 \times 10^{-7}$ \\
\hline MWV-02 & 3.0 & $8.2 \times 10^{-5}$ & $1.3 \times 10^{-7}$ & 1.6 & $4.4 \times 10^{-5}$ & $7.0 \times 10^{-8}$ & 4.5 & $1.2 \times 10^{-4}$ & $2.0 \times 10^{-7}$ \\
\hline MWV-09 & 7.0 & $1.9 \times 10^{-4}$ & $3.1 \times 10^{-7}$ & - & - & - & 1.1 & $3.0 \times 10^{-5}$ & $4.7 \times 10^{-8}$ \\
\hline MWV-13 & 3.2 & $8.8 \times 10^{-5}$ & $1.4 \times 10^{-7}$ & _ & - & - & 2.2 & $5.9 \times 10^{-5}$ & $9.5 \times 10^{-8}$ \\
\hline$M W V-16$ & 1.2 & $3.3 \times 10^{-5}$ & $5.3 \times 10^{-8}$ & 1.1 & $3.0 \times 10^{-5}$ & $4.8 \times 10^{-8}$ & 1.7 & $4.7 \times 10^{-5}$ & $7.6 \times 10^{-8}$ \\
\hline MWV-17 & 0.26 & $7.2 \times 10^{-6}$ & $1.2 \times 10^{-8}$ & - & - & - & 0.091 & $2.5 \times 10^{-6}$ & $4.0 \times 10^{-9}$ \\
\hline MWV-18 & - & - & - & - & - & - & - & - & - \\
\hline MWV-22 & 2.3 & $6.3 \times 10^{-5}$ & $1.0 \times 10^{-7}$ & 0.49 & $1.3 \times 10^{-5}$ & $2.1 \times 10^{-8}$ & 1.3 & $3.6 \times 10^{-5}$ & $5.8 \times 10^{-8}$ \\
\hline MWV-24R & 8.2 & $2.2 \times 10^{-4}$ & $3.6 \times 10^{-7}$ & 1.1 & $3.0 \times 10^{-5}$ & $4.8 \times 10^{-8}$ & 2.3 & $6.2 \times 10^{-5}$ & $9.9 \times 10^{-8}$ \\
\hline
\end{tabular}


TABLE 3.5 (Cont.)

\begin{tabular}{|c|c|c|c|c|c|c|c|c|c|}
\hline \multirow[b]{3}{*}{ Well ID } & \multicolumn{3}{|c|}{ Lithium } & \multicolumn{3}{|c|}{ Molybdenum } & \multicolumn{3}{|c|}{ Uranium, Total } \\
\hline & \multirow{2}{*}{$\begin{array}{c}\mathrm{EPC}^{\mathrm{a}} \\
(\mu \mathrm{g} / \mathrm{L})\end{array}$} & \multicolumn{2}{|c|}{ Intake (mg/kg-d) } & \multirow{2}{*}{$\begin{array}{l}\mathrm{EPC}^{\mathrm{a}} \\
(\mu \mathrm{g} / \mathrm{L})\end{array}$} & \multicolumn{2}{|c|}{ Intake (mg/kg-d) } & \multirow{2}{*}{$\begin{array}{c}\mathrm{EPC}^{\mathrm{a}} \\
(\mu \mathrm{g} / \mathrm{L})\end{array}$} & \multicolumn{2}{|c|}{ Intake (mg/kg-d) } \\
\hline & & Ingestion & Dermal & & Ingestion & Dermal & & Ingestion & Dermal \\
\hline \multicolumn{10}{|l|}{ Unweathered } \\
\hline MW-2019 & 21 & $5.8 \times 10^{-4}$ & $9.2 \times 10^{-7}$ & 27 & $7.4 \times 10^{-4}$ & $1.2 \times 10^{-6}$ & 4.5 & $1.2 \times 10^{-4}$ & $2.0 \times 10^{-7}$ \\
\hline MW-2021 & 3.8 & $1.0 \times 10^{-4}$ & $1.7 \times 10^{-7}$ & 7.6 & $2.1 \times 10^{-4}$ & $3.3 \times 10^{-7}$ & 1.3 & $3.6 \times 10^{-5}$ & $5.7 \times 10^{-8}$ \\
\hline MW-2022 & 3.7 & $1.0 \times 10^{-4}$ & $1.6 \times 10^{-7}$ & 2.3 & $6.3 \times 10^{-5}$ & $1.0 \times 10^{-7}$ & 1.9 & $5.1 \times 10^{-5}$ & $8.2 \times 10^{-8}$ \\
\hline MW-2023 & 3.6 & $9.9 \times 10^{-5}$ & $1.6 \times 10^{-7}$ & 6.6 & $1.8 \times 10^{-4}$ & $2.9 \times 10^{-7}$ & 3.8 & $1.0 \times 10^{-4}$ & $1.7 \times 10^{-7}$ \\
\hline MW-2024 & 5.3 & $1.5 \times 10^{-4}$ & $2.3 \times 10^{-7}$ & 2.0 & $5.5 \times 10^{-5}$ & $8.8 \times 10^{-8}$ & 0.17 & $4.6 \times 10^{-6}$ & $7.3 \times 10^{-9}$ \\
\hline MW-2025 & - & - & - & - & - & - & - & - & - \\
\hline MW-2026 & 29 & $7.9 \times 10^{-5}$ & $1.3 \times 10^{-7}$ & 8.2 & $2.2 \times 10^{-4}$ & $3.6 \times 10^{-7}$ & 1.2 & $3.3 \times 10^{-5}$ & $5.3 \times 10^{-8}$ \\
\hline $\mathrm{MW}-2027$ & 4.1 & $1.1 \times 10^{-4}$ & $1.8 \times 10^{-7}$ & 3.2 & $8.8 \times 10^{-5}$ & $1.4 \times 10^{-7}$ & 1.2 & $3.3 \times 10^{-5}$ & $5.3 \times 10^{-8}$ \\
\hline MW-2028 & 1.9 & $5.3 \times 10^{-4}$ & $8.5 \times 10^{-7}$ & 4.7 & $1.3 \times 10^{-4}$ & $2.1 \times 10^{-7}$ & 1.9 & $5.2 \times 10^{-5}$ & $8.3 \times 10^{-8}$ \\
\hline MW-2029 & - & - & - & - & - & - & - & - & - \\
\hline MW-3002 & - & - & - & - & - & - & - & - & - \\
\hline MW-3006 & 13 & $3.4 \times 10^{-4}$ & $5.5 \times 10^{-7}$ & 15 & $4.0 \times 10^{-4}$ & $6.4 \times 10^{-7}$ & 1.0 & $2.9 \times 10^{-5}$ & $4.6 \times 10^{-8}$ \\
\hline MW-3024 & 200 & $5.5 \times 10^{-3}$ & $8.9 \times 10^{-6}$ & - & - & - & 4.6 & $1.2 \times 10^{-4}$ & $2.0 \times 10^{-7}$ \\
\hline MW-3026 & 35 & $9.6 \times 10^{-4}$ & $1.5 \times 10^{-6}$ & 2.1 & $5.8 \times 10^{-5}$ & $9.2 \times 10^{-8}$ & 6.3 & $1.7 \times 10^{-4}$ & $2.8 \times 10^{-7}$ \\
\hline MW-4004 & 4.0 & $1.1 \times 10^{-4}$ & $1.8 \times 10^{-7}$ & 4.7 & $1.3 \times 10^{-4}$ & $2.1 \times 10^{-7}$ & 3.2 & $8.8 \times 10^{-5}$ & $1.4 \times 10^{-7}$ \\
\hline MW-4007 & 6.0 & $1.6 \times 10^{-4}$ & $2.6 \times 10^{-7}$ & 5.8 & $1.6 \times 10^{-4}$ & $2.5 \times 10^{-7}$ & 2.6 & $7.2 \times 10^{-5}$ & $1.2 \times 10^{-7}$ \\
\hline MW-4008 & 2.6 & $7.1 \times 10^{-5}$ & $1.1 \times 10^{-7}$ & - & - & - & 1.2 & $3.4 \times 10^{-5}$ & $5.4 \times 10^{-8}$ \\
\hline MW-4009 & 90 & $2.5 \times 10^{-3}$ & $3.9 \times 10^{-6}$ & 8.3 & $2.3 \times 10^{-4}$ & $3.6 \times 10^{-7}$ & 2.6 & $7.0 \times 10^{-5}$ & $1.1 \times 10^{-7}$ \\
\hline MW-4011 & 65 & $1.8 \times 10^{-3}$ & $2.8 \times 10^{-6}$ & 3.2 & $8.8 \times 10^{-5}$ & $1.4 \times 10^{-7}$ & 4.7 & $1.3 \times 10^{-4}$ & $2.0 \times 10^{-7}$ \\
\hline MW-4012 & 84 & $2.3 \times 10^{-3}$ & $3.7 \times 10^{-6}$ & 37 & $1.0 \times 10^{-3}$ & $1.6 \times 10^{-6}$ & 7.5 & $2.0 \times 10^{-4}$ & $3.3 \times 10^{-7}$ \\
\hline MW-4022 & 34 & $9.3 \times 10^{-4}$ & $1.5 \times 10^{-6}$ & 6.6 & $1.8 \times 10^{-4}$ & $2.9 \times 10^{-7}$ & 7.7 & $2.1 \times 10^{-4}$ & $3.4 \times 10^{-7}$ \\
\hline MWD-02 & 30 & $8.3 \times 10^{-4}$ & $1.3 \times 10^{-6}$ & 8.0 & $2.2 \times 10^{-4}$ & $3.5 \times 10^{-7}$ & 3.8 & $1.0 \times 10^{-4}$ & $1.7 \times 10^{-7}$ \\
\hline MWD-06 & 4.1 & $1.1 \times 10^{-4}$ & $1.8 \times 10^{-7}$ & - & - & - & 0.86 & $2.4 \times 10^{-5}$ & $3.8 \times 10^{-8}$ \\
\hline MWD-09 & 5.4 & $1.5 \times 10^{-4}$ & $2.4 \times 10^{-7}$ & - & - & - & 1.4 & $3.8 \times 10^{-5}$ & $6.1 \times 10^{-8}$ \\
\hline MWD-23 & 4.3 & $1.2 \times 10^{-4}$ & $1.9 \times 10^{-7}$ & 24 & $6.6 \times 10^{-4}$ & $1.1 \times 10^{-6}$ & 7.4 & $2.0 \times 10^{-4}$ & $3.2 \times 10^{-7}$ \\
\hline MWD-106 & 3.6 & $9.9 \times 10^{-5}$ & $1.6 \times 10^{-7}$ & - & - & - & - & - & - \\
\hline MWS-05 & 3.0 & $8.2 \times 10^{-5}$ & $1.3 \times 10^{-7}$ & - & - & - & 1.5 & $4.1 \times 10^{-5}$ & $6.5 \times 10^{-8}$ \\
\hline MWS-06 & 4.3 & $1.2 \times 10^{-4}$ & $1.9 \times 10^{-7}$ & - & - & - & 4.3 & $1.2 \times 10^{-4}$ & $1.9 \times 10^{-7}$ \\
\hline MWS-105 & 2.0 & $5.5 \times 10^{-5}$ & $8.8 \times 10^{-8}$ & 4.4 & $1.2 \times 10^{-4}$ & $1.9 \times 10^{-7}$ & 0.25 & $6.7 \times 10^{-6}$ & $1.1 \times 10^{-8}$ \\
\hline
\end{tabular}


TABLE 3.5 (Cont.)

\begin{tabular}{|c|c|c|c|c|c|c|c|c|c|}
\hline \multirow[b]{3}{*}{ Well ID } & \multicolumn{3}{|c|}{ Lithium } & \multicolumn{3}{|c|}{ Molybdenum } & \multicolumn{3}{|c|}{ Uranium, Total } \\
\hline & \multirow{2}{*}{$\begin{array}{l}\mathrm{EPC}^{\mathrm{a}} \\
(\mu \mathrm{g} / \mathrm{L})\end{array}$} & \multicolumn{2}{|c|}{ Intake (mg/kg-d) } & \multirow{2}{*}{$\begin{array}{c}\mathrm{EPC}^{\mathrm{a}} \\
(\mu \mathrm{g} / \mathrm{L})\end{array}$} & \multicolumn{2}{|c|}{ Intake (mg/kg-d) } & \multirow{2}{*}{$\begin{array}{c}\mathrm{EPC}^{\mathrm{a}} \\
(\mu \mathrm{g} / \mathrm{L})\end{array}$} & \multicolumn{2}{|c|}{ Intake (mg/kg-d) } \\
\hline & & Ingcstion & Dermal & & Ingestion & Dermal & & Ingestion & Dermal \\
\hline \multicolumn{10}{|c|}{ Unweathered (cont.) } \\
\hline MWS-106 & 2.3 & $6.3 \times 10^{-5}$ & $1.0 \times 10^{-7}$ & 2.3 & $6.3 \times 10^{-5}$ & $1.0 \times 10^{-7}$ & 1.7 & $4.7 \times 10^{-5}$ & $7.6 \times 10^{-8}$ \\
\hline MWS-109 & 2.2 & $6.0 \times 10^{-5}$ & $9.6 \times 10^{-8}$ & 1.9 & $5.2 \times 10^{-5}$ & $8.3 \times 10^{-8}$ & 1.5 & $4.1 \times 10^{-5}$ & $6.6 \times 10^{-8}$ \\
\hline TIL-4 & - & - & - & - & - & - & - & - & - \\
\hline USGS-1 & 3.8 & $1.0 \times 10^{-4}$ & $1.7 \times 10^{-7}$ & - & - & - & 1.6 & $4.3 \times 10^{-5}$ & $6.9 \times 10^{-8}$ \\
\hline USGS-6 & 1.8 & $4.9 \times 10^{-5}$ & $7.9 \times 10^{-8}$ & 3.2 & $8.8 \times 10^{-5}$ & $1.4 \times 10^{-7}$ & 5.9 & $1.6 \times 10^{-4}$ & $2.6 \times 10^{-7}$ \\
\hline \multicolumn{10}{|l|}{ Weathered } \\
\hline MW-2001 & 2.6 & $7.1 \times 10^{-5}$ & $1.1 \times 10^{-7}$ & - & - & - & 0.97 & $2.6 \times 10^{-5}$ & $4.2 \times 10^{-8}$ \\
\hline MW-2002 & 270 & $7.4 \times 10^{-3}$ & $1.2 \times 10^{-5}$ & 11 & $3.0 \times 10^{-4}$ & $4.8 \times 10^{-7}$ & 0.71 & $1.9 \times 10^{-5}$ & $3.1 \times 10^{-8}$ \\
\hline MW-2003 & 430 & $1.2 \times 10^{-2}$ & $1.9 \times 10^{-5}$ & 2.2 & $6.0 \times 10^{-5}$ & $9.6 \times 10^{-8}$ & 1.6 & $4.3 \times 10^{-5}$ & $6.9 \times 10^{-8}$ \\
\hline MW-2004 & - & - & - & - & - & - & - & - & - \\
\hline MW-2005 & 98 & $2.7 \times 10^{-3}$ & $4.3 \times 10^{-6}$ & 1.4 & $3.8 \times 10^{-5}$ & $6.1 \times 10^{-8}$ & 0.67 & $1.8 \times 10^{-5}$ & $3.0 \times 10^{-8}$ \\
\hline MW-2006 & 16 & $4.3 \times 10^{-4}$ & $6.9 \times 10^{-7}$ & 2.4 & $6.6 \times 10^{-5}$ & $1.1 \times 10^{-7}$ & 0.72 & $2.0 \times 10^{-5}$ & $3.2 \times 10^{-8}$ \\
\hline MW-2007 & 4.0 & $1.1 \times 10^{-4}$ & $1.8 \times 10^{-7}$ & 4.2 & $1.2 \times 10^{-4}$ & $1.8 \times 10^{-7}$ & 1.5 & $4.1 \times 10^{-5}$ & $6.6 \times 10^{-8}$ \\
\hline MW-2008 & - & - & - & - & - & - & - & - & - \\
\hline MW-2009 & - & - & - & - & - & - & - & - & - \\
\hline MW-2010 & 17 & $4.7 \times 10^{-4}$ & $7.5 \times 10^{-7}$ & 11 & $2.9 \times 10^{-4}$ & $4.6 \times 10^{-7}$ & 1.8 & $4.9 \times 10^{-5}$ & $7.9 \times 10^{-8}$ \\
\hline MW-2011 & 6.4 & $1.8 \times 10^{-4}$ & $2.8 \times 10^{-7}$ & - & - & - & 0.44 & $1.2 \times 10^{-5}$ & $1.9 \times 10^{-8}$ \\
\hline MW-2012 & 1.8 & $4.9 \times 10^{-5}$ & $7.9 \times 10^{-8}$ & - & - & - & 0.50 & $1.4 \times 10^{-5}$ & $2.2 \times 10^{-8}$ \\
\hline MW-2013 & 6.1 & $1.7 \times 10^{-4}$ & $2.7 \times 10^{-7}$ & - & - & - & 0.98 & $2.7 \times 10^{-5}$ & $4.3 \times 10^{-8}$ \\
\hline MW-2014 & 20 & $5.5 \times 10^{-4}$ & $8.9 \times 10^{-7}$ & - & - & - & 0.72 & $2.0 \times 10^{-5}$ & $3.2 \times 10^{-8}$ \\
\hline MW-2015 & 15 & $4.2 \times 10^{-4}$ & $6.7 \times 10^{-7}$ & - & - & - & 2.9 & $7.9 \times 10^{-5}$ & $1.3 \times 10^{-7}$ \\
\hline MW-2016 & - & - & - & - & - & - & - & - & - \\
\hline MW-2017 & 110 & $3.0 \times 10^{-3}$ & $4.7 \times 10^{-6}$ & 16 & $4.3 \times 10^{-4}$ & $6.8 \times 10^{-7}$ & 18 & $4.9 \times 10^{-4}$ & $7.9 \times 10^{-7}$ \\
\hline MW-2018 & 20 & $5.5 \times 10^{-4}$ & $8.8 \times 10^{-7}$ & 4.5 & $1.2 \times 10^{-4}$ & $2.0 \times 10^{-7}$ & 2.3 & $6.3 \times 10^{-5}$ & $1.0 \times 10^{-7}$ \\
\hline MW-2020 & - & - & - & - & - & - & - & - & - \\
\hline MW-2030 & 6.4 & $1.8 \times 10^{-4}$ & $2.8 \times 10^{-7}$ & - & - & - & 19 & $5.2 \times 10^{-4}$ & $8.2 \times 10^{-7}$ \\
\hline$M W-2034$ & 32 & $8.9 \times 10^{-4}$ & $1.4 \times 10^{-6}$ & - & - & - & 4.5 & $1.2 \times 10^{-4}$ & $2.0 \times 10^{-7}$ \\
\hline$M W-2035$ & 2.7 & $7.4 \times 10^{-5}$ & $1.2 \times 10^{-7}$ & 3.4 & $9.3 \times 10^{-5}$ & $1.5 \times 10^{-7}$ & 0.60 & $1.6 \times 10^{-5}$ & $2.6 \times 10^{-8}$ \\
\hline MW-2036 & 6.8 & $1.9 \times 10^{-4}$ & $3.0 \times 10^{-7}$ & - & - & - & 1.1 & $3.1 \times 10^{-5}$ & $5.0 \times 10^{-8}$ \\
\hline
\end{tabular}


TABLE 3.5 (Cont.)

\begin{tabular}{|c|c|c|c|c|c|c|c|c|c|}
\hline \multirow[b]{3}{*}{ Well ID } & \multicolumn{3}{|c|}{ Lithium } & \multicolumn{3}{|c|}{ Molybdenum } & \multicolumn{3}{|c|}{ Uranium, Total } \\
\hline & \multirow[b]{2}{*}{$\begin{array}{c}\mathrm{EPC}^{\mathrm{a}} \\
(\mu \mathrm{g} / \mathrm{L})\end{array}$} & \multicolumn{2}{|c|}{ Intake (mg/kg-d) } & \multirow[b]{2}{*}{$\begin{array}{c}\mathrm{EPC}^{\mathrm{a}} \\
(\mu \mathrm{g} / \mathrm{L})\end{array}$} & \multicolumn{2}{|c|}{ Intake $(\mathrm{mg} / \mathrm{kg}-\mathrm{d})$} & \multirow[b]{2}{*}{$\begin{array}{c}\mathrm{EPC}^{\mathrm{a}} \\
(\mu \mathrm{g} / \mathrm{L})\end{array}$} & \multicolumn{2}{|c|}{ Intake $(\mathrm{mg} / \mathrm{kg}-\mathrm{d})$} \\
\hline & & Ingestion & Dermal & & Ingestion & Dermal & & Ingestion & Dermal \\
\hline \multicolumn{10}{|c|}{ Weathered (cont.) } \\
\hline MW-2037 & 410 & $1.1 \times 10^{-2}$ & $1.8 \times 10^{-5}$ & - & - & - & 1.9 & $5.1 \times 10^{-5}$ & $8.1 \times 10^{-8}$ \\
\hline MW-2038 & 520 & $1.4 \times 10^{-2}$ & $2.3 \times 10^{-5}$ & 1.4 & $3.8 \times 10^{-5}$ & $6.1 \times 10^{-8}$ & 2.2 & $5.9 \times 10^{-5}$ & $9.5 \times 10^{-8}$ \\
\hline MW-2039 & 22 & $6.1 \times 10^{-4}$ & $9.7 \times 10^{-7}$ & 4.4 & $1.2 \times 10^{-4}$ & $1.9 \times 10^{-7}$ & 4.6 & $1.3 \times 10^{-4}$ & $2.0 \times 10^{-7}$ \\
\hline $\mathrm{MW}-2040$ & 33 & $9.0 \times 10^{-4}$ & $1.4 \times 10^{-6}$ & 6.6 & $1.8 \times 10^{-4}$ & $2.9 \times 10^{-7}$ & 4.5 & $1.2 \times 10^{-4}$ & $2.0 \times 10^{-7}$ \\
\hline MW-2041 & 26 & $7.0 \times 10^{-4}$ & $1.1 \times 10^{-6}$ & 2.2 & $6.0 \times 10^{-5}$ & $9.6 \times 10^{-8}$ & 5.0 & $1.4 \times 10^{-4}$ & $2.2 \times 10^{-7}$ \\
\hline MW-2042 & 20 & $5.6 \times 10^{-4}$ & $8.9 \times 10^{-7}$ & - & - & - & 3.9 & $1.1 \times 10^{-4}$ & $1.7 \times 10^{-7}$ \\
\hline MW-2043 & 17 & $4.7 \times 10^{-4}$ & $7.5 \times 10^{-7}$ & 1.5 & $4.1 \times 10^{-5}$ & $6.6 \times 10^{-8}$ & 2.7 & $7.3 \times 10^{-5}$ & $1.2 \times 10^{-7}$ \\
\hline MW-2044 & 29 & $7.9 \times 10^{-4}$ & $1.3 \times 10^{-6}$ & 1.8 & $4.9 \times 10^{-5}$ & $7.9 \times 10^{-8}$ & 3.4 & $9.2 \times 10^{-5}$ & $1.5 \times 10^{-7}$ \\
\hline MW-3003 & 650 & $1.8 \times 10^{-2}$ & $2.8 \times 10^{-5}$ & 5.7 & $1.6 \times 10^{-4}$ & $2.5 \times 10^{-7}$ & 28 & $7.6 \times 10^{-4}$ & $1.2 \times 10^{-6}$ \\
\hline MW-3007 & - & - & - & - & - & - & - & - & - \\
\hline MW-3008 & - & - & - & - & - & - & - & - & - \\
\hline MW-3009 & - & - & - & - & - & - & - & - & - \\
\hline MW-3010 & - & - & - & - & - & - & - & - & - \\
\hline MW-3019 & 15 & $4.0 \times 10^{-4}$ & $6.4 \times 10^{-7}$ & 1.0 & $2.7 \times 10^{-5}$ & $4.4 \times 10^{-8}$ & 3.2 & $8.7 \times 10^{-5}$ & $1.4 \times 10^{-7}$ \\
\hline MW-3023 & 640 & $1.8 \times 10^{-2}$ & $2.8 \times 10^{-5}$ & 250 & $6.9 \times 10^{-3}$ & $1.1 \times 10^{-5}$ & 19 & $5.2 \times 10^{-4}$ & $8.4 \times 10^{-7}$ \\
\hline MW-3025 & 160 & $4.4 \times 10^{-3}$ & $7.0 \times 10^{-6}$ & - & - & - & 4.1 & $1.1 \times 10^{-4}$ & $1.8 \times 10^{-7}$ \\
\hline $\mathrm{MW}-3027$ & 18 & $4.9 \times 10^{-4}$ & $7.9 \times 10^{-7}$ & - & - & - & 1.9 & $5.2 \times 10^{-5}$ & $8.2 \times 10^{-8}$ \\
\hline MW-4001 & 7.7 & $2.1 \times 10^{-4}$ & $3.4 \times 10^{-7}$ & 1.8 & $4.9 \times 10^{-5}$ & $7.9 \times 10^{-8}$ & 0.61 & $1.7 \times 10^{-5}$ & $2.7 \times 10^{-8}$ \\
\hline MW-4002 & 3.9 & $1.1 \times 10^{-4}$ & $1.7 \times 10^{-7}$ & 1.1 & $3.0 \times 10^{-5}$ & $4.8 \times 10^{-8}$ & 0.89 & $2.4 \times 10^{-5}$ & $3.9 \times 10^{-8}$ \\
\hline MW-4003 & 3.0 & $8.2 \times 10^{-5}$ & $1.3 \times 10^{-7}$ & - & - & - & 1.7 & $4.7 \times 10^{-5}$ & $7.5 \times 10^{-8}$ \\
\hline MW-4005 & 6.7 & $1.8 \times 10^{-4}$ & $2.9 \times 10^{-7}$ & 5.0 & $1.4 \times 10^{-4}$ & $2.2 \times 10^{-7}$ & 2.4 & $6.5 \times 10^{-5}$ & $1.0 \times 10^{-7}$ \\
\hline MW-4006 & 3.0 & $8.2 \times 10^{-5}$ & $1.3 \times 10^{-7}$ & 1.1 & $3.0 \times 10^{-5}$ & $4.8 \times 10^{-8}$ & 0.39 & $1.1 \times 10^{-5}$ & $1.7 \times 10^{-8}$ \\
\hline MW-4010 & 5.8 & $1.6 \times 10^{-4}$ & $2.5 \times 10^{-7}$ & 3.7 & $1.0 \times 10^{-4}$ & $1.6 \times 10^{-7}$ & 4.6 & $1.3 \times 10^{-4}$ & $2.0 \times 10^{-7}$ \\
\hline MW-4013 & 68 & $1.9 \times 10^{-3}$ & $3.0 \times 10^{-6}$ & - & - & - & 1.8 & $4.8 \times 10^{-5}$ & $7.7 \times 10^{-8}$ \\
\hline MW-4014 & 3.5 & $9.6 \times 10^{-5}$ & $1.5 \times 10^{-7}$ & - & - & - & 0.33 & $9.1 \times 10^{-6}$ & $1.5 \times 10^{-8}$ \\
\hline MW-4015 & 1.9 & $5.2 \times 10^{-5}$ & $8.3 \times 10^{-8}$ & 0.25 & $6.8 \times 10^{-6}$ & $1.1 \times 10^{-8}$ & 0.48 & $1.3 \times 10^{-5}$ & $2.1 \times 10^{-8}$ \\
\hline MW-4016 & 3.7 & $1.0 \times 10^{-4}$ & $1.6 \times 10^{-7}$ & 9.6 & $2.6 \times 10^{-4}$ & $4.2 \times 10^{-7}$ & 4.7 & $1.3 \times 10^{-4}$ & $2.1 \times 10^{-7}$ \\
\hline MW-4017 & - & - & - & - & - & - & - & - & - \\
\hline MW-4018 & 4.1 & $1.1 \times 10^{-4}$ & $1.8 \times 10^{-7}$ & - & - & - & 0.95 & $2.6 \times 10^{-5}$ & $4.2 \times 10^{-8}$ \\
\hline MW-4019 & 10 & $2.7 \times 10^{-4}$ & $4.4 \times 10^{-7}$ & - & - & - & 2.6 & $7.0 \times 10^{-5}$ & $1.1 \times 10^{-7}$ \\
\hline
\end{tabular}


TABLE 3.5 (Cont.)

\begin{tabular}{|c|c|c|c|c|c|c|c|c|c|}
\hline \multirow[b]{3}{*}{ Well ID } & \multicolumn{3}{|c|}{ Lithium } & \multicolumn{3}{|c|}{ Molybdenum } & \multicolumn{3}{|c|}{ Uranium, Total } \\
\hline & \multirow{2}{*}{$\begin{array}{c}\mathrm{EPC}^{\mathrm{a}} \\
(\mu \mathrm{g} / \mathrm{L})\end{array}$} & \multicolumn{2}{|c|}{ Intake $(\mathrm{mg} / \mathrm{kg}-\mathrm{d})$} & \multirow{2}{*}{$\begin{array}{l}\mathrm{EPC}^{\mathrm{a}} \\
(\mu \mathrm{g} / \mathrm{L})\end{array}$} & \multicolumn{2}{|c|}{ Intake (mg/kg-d) } & \multirow{2}{*}{$\begin{array}{l}\mathrm{EPC}^{\mathrm{a}} \\
(\mu \mathrm{g} / \mathrm{L})\end{array}$} & \multicolumn{2}{|c|}{ Intake (mg/kg-d) } \\
\hline & & Ingestion & Dermal & & Ingestion & Dermal & & Ingestion & Dermal \\
\hline \multicolumn{10}{|c|}{ Weathered (cont.) } \\
\hline MW-4020 & 22 & $6.1 \times 10^{-4}$ & $9.8 \times 10^{-7}$ & 1.3 & $3.6 \times 10^{-5}$ & $5.7 \times 10^{-8}$ & 15 & $4.0 \times 10^{-4}$ & $6.4 \times 10^{-7}$ \\
\hline MW-4021 & 23 & $6.2 \times 10^{-4}$ & $9.9 \times 10^{-7}$ & - & - & - & 4.6 & $1.3 \times 10^{-4}$ & $2.0 \times 10^{-7}$ \\
\hline MW-4023 & 14 & $3.9 \times 10^{-4}$ & $6.3 \times 10^{-7}$ & - & - & - & 2.3 & $6.4 \times 10^{-5}$ & $1.0 \times 10^{-7}$ \\
\hline MW-4024 & 82 & $2.2 \times 10^{-3}$ & $3.6 \times 10^{-6}$ & 11 & $3.0 \times 10^{-4}$ & $4.9 \times 10^{-7}$ & 90 & $2.5 \times 10^{-3}$ & $3.9 \times 10^{-6}$ \\
\hline MW-4025 & 12 & $3.2 \times 10^{-4}$ & $5.1 \times 10^{-7}$ & 4.2 & $1.2 \times 10^{-4}$ & $1.8 \times 10^{-7}$ & 1.5 & $4.2 \times 10^{-5}$ & $6.7 \times 10^{-8}$ \\
\hline MWD-15 & 1.2 & $3.3 \times 10^{-5}$ & $5.3 \times 10^{-8}$ & - & - & - & 0.74 & $2.0 \times 10^{-5}$ & $3.2 \times 10^{-8}$ \\
\hline MWD-25 & 2.4 & $6.6 \times 10^{-5}$ & $1.1 \times 10^{-7}$ & 1.9 & $5.2 \times 10^{-5}$ & $8.3 \times 10^{-8}$ & 2.6 & $7.2 \times 10^{-5}$ & $1.2 \times 10^{-7}$ \\
\hline MWD-107 & 5.4 & $1.5 \times 10^{-4}$ & $2.4 \times 10^{-7}$ & 4.6 & $1.3 \times 10^{-4}$ & $2.0 \times 10^{-7}$ & 3.0 & $8.3 \times 10^{-5}$ & $1.3 \times 10^{-7}$ \\
\hline MWD -112 & 2.7 & $7.4 \times 10^{-5}$ & $1.2 \times 10^{-7}$ & 2.2 & $6.0 \times 10^{-5}$ & $9.6 \times 10^{-8}$ & 1.2 & $3.2 \times 10^{-5}$ & $5.1 \times 10^{-8}$ \\
\hline MWS-01 & 1.9 & $5.2 \times 10^{-5}$ & $8.3 \times 10^{-8}$ & - & - & - & 1.9 & $5.3 \times 10^{-5}$ & $8.5 \times 10^{-8}$ \\
\hline MWS-02 & 3.6 & $9.9 \times 10^{-5}$ & $1.6 \times 10^{-7}$ & 4.2 & $1.2 \times 10^{-4}$ & $1.8 \times 10^{-7}$ & 3.0 & $8.3 \times 10^{-5}$ & $1.3 \times 10^{-7}$ \\
\hline MWS-03 & 5.1 & $1.4 \times 10^{-4}$ & $2.2 \times 10^{-7}$ & 3.4 & $9.3 \times 10^{-5}$ & $1.5 \times 10^{-7}$ & 4.9 & $1.3 \times 10^{-4}$ & $2.1 \times 10^{-7}$ \\
\hline MWS-04 & 4.0 & $1.1 \times 10^{-4}$ & $1.8 \times 10^{-7}$ & - & - & - & 15 & $4.2 \times 10^{-4}$ & $6.7 \times 10^{-7}$ \\
\hline MWS-07 & - & - & - & - & - & - & 1.1 & $3.0 \times 10^{-5}$ & $4.7 \times 10^{-8}$ \\
\hline MWS-08 & 23 & $6.2 \times 10^{-4}$ & $9.9 \times 10^{-7}$ & - & - & - & 1.7 & $4.6 \times 10^{-5}$ & $7.3 \times 10^{-8}$ \\
\hline MWS-09 & 6.6 & $1.8 \times 10^{-4}$ & $2.9 \times 10^{-7}$ & - & - & - & 1.8 & $4.9 \times 10^{-5}$ & $7.8 \times 10^{-8}$ \\
\hline MWS-10 & 4.5 & $1.2 \times 10^{-4}$ & $2.0 \times 10^{-7}$ & 1.7 & $4.7 \times 10^{-5}$ & $7.5 \times 10^{-8}$ & 0.20 & $5.4 \times 10^{-6}$ & $8.7 \times 10^{-9}$ \\
\hline MWS-11 & 2.4 & $6.6 \times 10^{-5}$ & $1.1 \times 10^{-7}$ & - & - & - & 2.6 & $7.0 \times 10^{-5}$ & $1.1 \times 10^{-7}$ \\
\hline MWS- 12 & 3.1 & $8.5 \times 10^{-5}$ & $1.4 \times 10^{-7}$ & - & - & - & 1.5 & $4.1 \times 10^{-5}$ & $6.5 \times 10^{-8}$ \\
\hline MWS-13 & 6.8 & $1.9 \times 10^{-4}$ & $3.0 \times 10^{-7}$ & 0.54 & $1.5 \times 10^{-5}$ & $2.3 \times 10^{-8}$ & 0.80 & $2.2 \times 10^{-5}$ & $3.5 \times 10^{-8}$ \\
\hline MWS-14 & 25 & $6.7 \times 10^{-4}$ & $1.1 \times 10^{-6}$ & 1.6 & $4.4 \times 10^{-5}$ & $7.0 \times 10^{-8}$ & 4.0 & $1.1 \times 10^{-4}$ & $1.8 \times 10^{-7}$ \\
\hline MWS-15 & 1.3 & $3.6 \times 10^{-5}$ & $5.7 \times 10^{-8}$ & - & - & - & 0.84 & $2.3 \times 10^{-5}$ & $3.7 \times 10^{-8}$ \\
\hline MWS-16 & - & - & - & - & - & - & 0.98 & $2.7 \times 10^{-5}$ & $4.3 \times 10^{-8}$ \\
\hline MWS- 17 & 2.6 & $7.1 \times 10^{-5}$ & $1.1 \times 10^{-7}$ & - & - & - & 1.7 & $4.8 \times 10^{-5}$ & $7.7 \times 10^{-8}$ \\
\hline MWS-19 & 1.2 & $3.3 \times 10^{-5}$ & $5.3 \times 10^{-8}$ & 1.0 & $2.7 \times 10^{-5}$ & $4.4 \times 10^{-8}$ & 1.9 & $5.3 \times 10^{-5}$ & $8.4 \times 10^{-8}$ \\
\hline MWS-20 & 1.5 & $4.0 \times 10^{-5}$ & $6.4 \times 10^{-8}$ & - & - & - & 1.0 & $2.8 \times 10^{-5}$ & $4.5 \times 10^{-8}$ \\
\hline MWS-21 & 0.36 & $9.8 \times 10^{-3}$ & $1.6 \times 10^{-5}$ & 4.9 & $1.3 \times 10^{-4}$ & $2.1 \times 10^{-7}$ & 4.5 & $1.2 \times 10^{-4}$ & $2.0 \times 10^{-7}$ \\
\hline MWS-22 & 3.0 & $8.2 \times 10^{-5}$ & $1.3 \times 10^{-7}$ & 0.13 & $3.5 \times 10^{-6}$ & $5.6 \times 10^{-9}$ & 1.8 & $4.9 \times 10^{-5}$ & $7.8 \times 10^{-8}$ \\
\hline MWS-24 & - & - & - & - & - & - & - & - & - \\
\hline MWS-25 & - & - & - & 1.7 & $4.7 \times 10^{-5}$ & $7.5 \times 10^{-8}$ & 2.4 & $6.5 \times 10^{-5}$ & $1.0 \times 10^{-7}$ \\
\hline
\end{tabular}


TABLE 3.5 (Cont.)

\begin{tabular}{|c|c|c|c|c|c|c|c|c|c|}
\hline \multirow[b]{3}{*}{ Well ID } & \multicolumn{3}{|c|}{ Lithium } & \multicolumn{3}{|c|}{ Molybdenum } & \multicolumn{3}{|c|}{ Uranium, Total } \\
\hline & \multirow{2}{*}{$\begin{array}{c}\mathrm{EPC}^{\mathrm{a}} \\
(\mu \mathrm{g} / \mathrm{L})\end{array}$} & \multicolumn{2}{|c|}{ Intake (mg/kg-d) } & \multirow{2}{*}{$\begin{array}{c}\mathrm{EPC}^{\mathrm{a}} \\
(\mu \mathrm{g} / \mathrm{L})\end{array}$} & \multicolumn{2}{|c|}{ Intake (mg/kg-d) } & \multirow{2}{*}{$\begin{array}{l}\mathrm{EPC}^{\mathrm{a}} \\
(\mu \mathrm{g} / \mathrm{L})\end{array}$} & \multicolumn{2}{|c|}{ Intake (mg/kg-d) } \\
\hline & & Ingestion & Dermal & & Ingestion & Dermal & & Ingestion & Dermal \\
\hline \multicolumn{10}{|c|}{ Weathered (cont.) } \\
\hline MWS-26 & 15 & $4.1 \times 10^{-4}$ & $6.6 \times 10^{-7}$ & 2.0 & $5.5 \times 10^{-5}$ & $8.8 \times 10^{-8}$ & 5.9 & $1.6 \times 10^{-4}$ & $2.6 \times 10^{-7}$ \\
\hline MWS-104 & 1.7 & $4.7 \times 10^{-5}$ & $7.5 \times 10^{-8}$ & 4.4 & $1.2 \times 10^{-4}$ & $1.9 \times 10^{-7}$ & 1.9 & $5.3 \times 10^{-5}$ & $8.5 \times 10^{-8}$ \\
\hline MWS-107 & 5.0 & $1.4 \times 10^{-4}$ & $2.2 \times 10^{-7}$ & 1.0 & $2.7 \times 10^{-5}$ & $4.4 \times 10^{-8}$ & 2.7 & $7.4 \times 10^{-5}$ & $1.2 \times 10^{-7}$ \\
\hline MWS-110 & 3.3 & $9.0 \times 10^{-5}$ & $1.4 \times 10^{-7}$ & - & - & - & 0.93 & $2.6 \times 10^{-5}$ & $4.1 \times 10^{-8}$ \\
\hline MWS-112 & 17 & $4.7 \times 10^{-4}$ & $7.5 \times 10^{-7}$ & 36 & $9.7 \times 10^{-4}$ & $1.6 \times 10^{-6}$ & 4.1 & $1.1 \times 10^{-4}$ & $1.8 \times 10^{-7}$ \\
\hline USGS-2 & - & - & - & 1.8 & $4.9 \times 10^{-5}$ & $7.9 \times 10^{-8}$ & 0.51 & $1.4 \times 10^{-5}$ & $6.5 \times 10^{-11}$ \\
\hline USGS-3 & 4.8 & $1.3 \times 10^{-4}$ & $2.1 \times 10^{-7}$ & 1.6 & $4.4 \times 10^{-5}$ & $7.0 \times 10^{-8}$ & 2.1 & $5.8 \times 10^{-5}$ & $9.3 \times 10^{-8}$ \\
\hline USGS-4 & 3.9 & $1.1 \times 10^{-4}$ & $1.7 \times 10^{-7}$ & 1.2 & $3.3 \times 10^{-5}$ & $5.3 \times 10^{-8}$ & 0.80 & $2.2 \times 10^{-5}$ & $3.5 \times 10^{-8}$ \\
\hline USGS-5 & 3.5 & $9.6 \times 10^{-5}$ & $1.5 \times 10^{-7}$ & 5.4 & $1.5 \times 10^{-4}$ & $2.4 \times 10^{-7}$ & 7.3 & $2.0 \times 10^{-4}$ & $3.2 \times 10^{-7}$ \\
\hline USGS-7 & - & - & - & - & - & - & - & - & - \\
\hline USGS-8 & 10 & $2.8 \times 10^{-4}$ & $4.5 \times 10^{-7}$ & 1.6 & $4.4 \times 10^{-5}$ & $7.0 \times 10^{-8}$ & 0.93 & $2.6 \times 10^{-5}$ & $4.1 \times 10^{-8}$ \\
\hline USGS-9 & 3.0 & $8.2 \times 10^{-5}$ & $1.3 \times 10^{-7}$ & - & - & - & 0.51 & $1.4 \times 10^{-5}$ & $2.3 \times 10^{-8}$ \\
\hline
\end{tabular}

a $\mathrm{EPC}=$ exposure point concentration, which is the maximum uranium value for each spring from the 1995 joint DOE/DA sampling rounds.

b A hyphen (-) indicates the parameter was not detected. 
TABLE 3.6 Estimated Noncarcinogenic Intakes of Inorganic Anion COPCs for the Hypothetical Future Resident

\begin{tabular}{|c|c|c|c|c|c|c|c|c|c|}
\hline \multirow[b]{3}{*}{ Well ID } & \multicolumn{3}{|c|}{ Chloride } & \multicolumn{3}{|c|}{ Nitrate-N } & \multicolumn{3}{|c|}{ Sulfate } \\
\hline & \multirow[b]{2}{*}{$\begin{array}{c}\mathrm{EPC}^{\mathrm{a}} \\
(\mathrm{mg} / \mathrm{L})\end{array}$} & \multicolumn{2}{|c|}{ Intake $(\mathrm{mg} / \mathrm{kg}-\mathrm{d})$} & \multirow[b]{2}{*}{$\begin{array}{c}\mathrm{EPC}^{\mathrm{a}} \\
(\mathrm{mg} / \mathrm{L})\end{array}$} & \multicolumn{2}{|c|}{ Intake $(\mathrm{mg} / \mathrm{kg}-\mathrm{d})$} & \multirow[b]{2}{*}{$\begin{array}{c}\mathrm{EPC}^{\mathrm{a}} \\
(\mathrm{mg} / \mathrm{L})\end{array}$} & \multicolumn{2}{|c|}{ Intake $(\mathrm{mg} / \mathrm{kg}-\mathrm{d})$} \\
\hline & & Ingestion & Dermal & & Ingestion $^{b}$ & Dermal & & Ingestion & Dermal \\
\hline \multicolumn{10}{|l|}{ Deep Wells } \\
\hline MWD-05 & 1.9 & $5.2 \times 10^{-2}$ & $8.3 \times 10^{-5}$ & 0.13 & $3.6 \times 10^{-3}$ & $5.7 \times 10^{-6}$ & 26 & $7.1 \times 10^{-1}$ & $1.1 \times 10^{-3}$ \\
\hline MWD-18 & 1.8 & $4.9 \times 10^{-2}$ & $7.9 \times 10^{-5}$ & - & - & - & 15 & $4.1 \times 10^{-1}$ & $6.6 \times 10^{-4}$ \\
\hline MWGS-01 & $-\mathrm{c}$ & - & - & - & - & - & - & - & - \\
\hline MWGS-02 & - & - & - & - & - & - & - & - & - \\
\hline MWS- 18 & 2.9 & $7.9 \times 10^{-2}$ & $1.3 \times 10^{-4}$ & 0.33 & $9.0 \times 10^{-3}$ & $1.4 \times 10^{-5}$ & 100 & 2.7 & $4.4 \times 10^{-3}$ \\
\hline MWS-101 & 9.1 & $2.5 \times 10^{-1}$ & $4.0 \times 10^{-4}$ & - & - & - & 8.4 & $2.3 \times 10^{-1}$ & $3.7 \times 10^{-4}$ \\
\hline MWS-102 & 4.2 & $1.2 \times 10^{-1}$ & $1.8 \times 10^{-4}$ & - & - & - & 15 & $4.1 \times 10^{-1}$ & $6.6 \times 10^{-4}$ \\
\hline MWS-103 & 3.2 & $8.8 \times 10^{-2}$ & $1.4 \times 10^{-4}$ & - & - & - & 49 & 1.3 & $2.1 \times 10^{-3}$ \\
\hline TIL-3 & 2.3 & $6.3 \times 10^{-2}$ & $1.0 \times 10^{-4}$ & 0.1 & - & - & 11 & $3.0 \times 10^{-1}$ & $4.8 \times 10^{-4}$ \\
\hline \multicolumn{10}{|l|}{ Overburden } \\
\hline MW-2031 & - & - & - & - & - & - & - & - & - \\
\hline MW-2032 & 17 & $4.7 \times 10^{-1}$ & $7.5 \times 10^{-4}$ & 56 & 1.5 & $2.5 \times 10^{-3}$ & 54 & 1.5 & $2.4 \times 10^{-3}$ \\
\hline MW-2033 & 5.4 & $1.5 \times 10^{-1}$ & $2.4 \times 10^{-4}$ & 1.1 & $3.0 \times 10^{-2}$ & $4.8 \times 10^{-5}$ & 42 & 1.2 & $1.8 \times 10^{-3}$ \\
\hline MW-3001 & - & - & - & - & - & - & - & - & - \\
\hline MW-3013 & - & - & - & - & - & - & - & - & - \\
\hline MW-3018 & - & - & - & - & - & - & - & - & - \\
\hline MW-3022 & - & - & - & - & - & - & - & - & - \\
\hline USGS-2A & - & - & - & - & - & - & - & - & - \\
\hline MWV-01 & - & - & - & 1.5 & $4.1 \times 10^{-2}$ & $6.6 \times 10^{-5}$ & 14 & $3.8 \times 10^{-1}$ & $6.1 \times 10^{-4}$ \\
\hline MWV-02 & 1.8 & $4.9 \times 10^{-2}$ & $7.9 \times 10^{-5}$ & 2.7 & $7.4 \times 10^{-2}$ & $1.2 \times 10^{-4}$ & 25 & $6.8 \times 10^{-1}$ & $1.1 \times 10^{-3}$ \\
\hline MWV-09 & 2.3 & $6.3 \times 10^{-2}$ & $1.0 \times 10^{-4}$ & 0.79 & $2.2 \times 10^{-2}$ & $3.5 \times 10^{-5}$ & 56 & 1.5 & $2.5 \times 10^{-3}$ \\
\hline MWV-13 & 6.6 & $1.8 \times 10^{-1}$ & $2.9 \times 10^{-4}$ & 1.5 & $4.1 \times 10^{-2}$ & $6.6 \times 10^{-5}$ & 360 & 9.9 & $1.6 \times 10^{-2}$ \\
\hline MWV-16 & 3 & $8.2 \times 10^{-2}$ & $1.3 \times 10^{-4}$ & 1.1 & $3.0 \times 10^{-2}$ & $4.8 \times 10^{-5}$ & 25 & $6.8 \times 10^{-1}$ & $1.1 \times 10^{-3}$ \\
\hline MWV-17 & - & - & - & 2.1 & $5.8 \times 10^{-2}$ & $9.2 \times 10^{-5}$ & 15 & $4.1 \times 10^{-1}$ & $6.6 \times 10^{-4}$ \\
\hline$M W V-18$ & - & - & - & - & - & - & - & - & - \\
\hline MWV-22 & 4 & $1.1 \times 10^{-1}$ & $1.8 \times 10^{-4}$ & 3.4 & $9.3 \times 10^{-2}$ & $1.5 \times 10^{-4}$ & 14 & $3.8 \times 10^{-1}$ & $6.1 \times 10^{-4}$ \\
\hline MWV-24R & 1.1 & $3.0 \times 10^{-2}$ & $4.8 \times 10^{-5}$ & 0.35 & $9.6 \times 10^{-3}$ & $1.5 \times 10^{-5}$ & 31 & $8.5 \times 10^{-1}$ & $1.4 \times 10^{-3}$ \\
\hline
\end{tabular}


TABLE 3.6 (Cont.)

\begin{tabular}{|c|c|c|c|c|c|c|c|c|c|}
\hline \multirow[b]{3}{*}{ Well ID } & \multicolumn{3}{|c|}{ Chloride } & \multicolumn{3}{|c|}{ Nitrate-N } & \multicolumn{3}{|c|}{ Sulfate } \\
\hline & \multirow[b]{2}{*}{$\begin{array}{c}\mathrm{EPC}^{\mathrm{a}} \\
(\mathrm{mg} / \mathrm{L})\end{array}$} & \multicolumn{2}{|c|}{ Intake (mg/kg-d) } & \multirow[b]{2}{*}{$\begin{array}{c}\mathrm{EPC}^{\mathrm{a}} \\
(\mathrm{mg} / \mathrm{L})\end{array}$} & \multicolumn{2}{|c|}{ Intake (mg/kg-d) } & \multirow[b]{2}{*}{$\begin{array}{c}\mathrm{EPC}^{\mathrm{a}} \\
(\mathrm{mg} / \mathrm{L}) \\
\end{array}$} & \multicolumn{2}{|c|}{ Intake (mg/kg-d) } \\
\hline & & Ingestion & Dermal & & Ingestion $^{b}$ & Dermal & & Ingestion & Dermal \\
\hline \multicolumn{10}{|l|}{ Unweathered } \\
\hline MW-2019 & 1 & $2.7 \times 10^{-2}$ & $4.4 \times 10^{-5}$ & - & - & - & 22 & $6.0 \times 10^{-1}$ & $9.6 \times 10^{-4}$ \\
\hline MW-2021 & 1.1 & $3.0 \times 10^{-2}$ & $4.8 \times 10^{-5}$ & 0.005 & $1.4 \times 10^{-4}$ & $2.2 \times 10^{-7}$ & 13 & $3.6 \times 10^{-1}$ & $5.7 \times 10^{-4}$ \\
\hline MW-2022 & 1.2 & $3.3 \times 10^{-2}$ & $5.3 \times 10^{-5}$ & - & - & - & 14 & $3.8 \times 10^{-1}$ & $6.1 \times 10^{-4}$ \\
\hline MW-2023 & 1.1 & $3.0 \times 10^{-2}$ & $4.8 \times 10^{-5}$ & - & - & - & 14 & $3.8 \times 10^{-1}$ & $6.1 \times 10^{-4}$ \\
\hline MW-2024 & 1.7 & $4.7 \times 10^{-2}$ & $7.5 \times 10^{-5}$ & - & - & - & 29 & $7.9 \times 10^{-1}$ & $1.3 \times 10^{-3}$ \\
\hline MW-2025 & - & - & - & - & - & - & - & - & - \\
\hline MW-2026 & 1.4 & $3.8 \times 10^{-2}$ & $6.1 \times 10^{-5}$ & - & - & - & 13 & $3.6 \times 10^{-1}$ & $5.7 \times 10^{-4}$ \\
\hline $\mathrm{MW}-2027$ & 1.1 & $3.0 \times 10^{-2}$ & $4.8 \times 10^{-5}$ & - & - & - & 5.3 & $1.5 \times 10^{-1}$ & $2.3 \times 10^{-4}$ \\
\hline MW-2028 & 1.3 & $3.6 \times 10^{-2}$ & $5.7 \times 10^{-5}$ & - & - & - & 130 & 3.4 & $5.5 \times 10^{-3}$ \\
\hline MW-2029 & - & - & - & - & - & - & - & - & - \\
\hline MW-3002 & - & - & - & - . & - & - & - & - & - \\
\hline MW-3006 & 1.2 & $3.3 \times 10^{-2}$ & $5.3 \times 10^{-5}$ & - & - & - & 22 & $6.0 \times 10^{-1}$ & $9.6 \times 10^{-4}$ \\
\hline MW-3024 & 12 & $3.3 \times 10^{-1}$ & $5.3 \times 10^{-4}$ & 370 & $1.0 \times 10^{1}$ & $1.6 \times 10^{-2}$ & 88 & 2.4 & $3.9 \times 10^{-3}$ \\
\hline MW-3026 & 6.3 & $1.7 \times 10^{-1}$ & $2.8 \times 10^{-4}$ & 220 & 6.0 & $9.6 \times 10^{-3}$ & 19 & $5.2 \times 10^{-1}$ & $8.3 \times 10^{-4}$ \\
\hline MW-4004 & 3.3 & $9.0 \times 10^{-2}$ & $1.4 \times 10^{-4}$ & 1.1 & $3.0 \times 10^{-2}$ & $4.8 \times 10^{-5}$ & 19 & $5.2 \times 10^{-1}$ & $8.3 \times 10^{-4}$ \\
\hline MW-4007 & 2.3 & $6.3 \times 10^{-2}$ & $1.0 \times 10^{-4}$ & - & - & - & 62 & 1.7 & $2.7 \times 10^{-3}$ \\
\hline MW-4008 & - & - & - & - & - & - & 14 & $3.8 \times 10^{-1}$ & $6.1 \times 10^{-4}$ \\
\hline MW-4009 & - & - & - & 0.14 & $3.8 \times 10^{-3}$ & $6.1 \times 10^{-6}$ & 13 & $3.6 \times 10^{-1}$ & $5.7 \times 10^{-4}$ \\
\hline MW-4011 & 11 & $3.0 \times 10^{-1}$ & $4.8 \times 10^{-4}$ & 170 & 4.7 & $7.5 \times 10^{-3}$ & 83 & 2.3 & $3.6 \times 10^{-3}$ \\
\hline $\mathrm{MW}-4012$ & 1.8 & $4.9 \times 10^{-2}$ & $7.9 \times 10^{-5}$ & - & - & - & 36 & $9.9 \times 10^{-1}$ & $1.6 \times 10^{-3}$ \\
\hline$M W-4022$ & 2.6 & $7.1 \times 10^{-2}$ & $1.1 \times 10^{-4}$ & 0.39 & $1.1 \times 10^{-2}$ & $1.7 \times 10^{-5}$ & 23 & $6.3 \times 10^{-1}$ & $1.0 \times 10^{-3}$ \\
\hline MWD-02 & 1.1 & $3.0 \times 10^{-2}$ & $4.8 \times 10^{-5}$ & - & - & - & 16 & $4.4 \times 10^{-1}$ & $7.0 \times 10^{-4}$ \\
\hline MWD-06 & - & - & - & - & - & - & 20 & $5.5 \times 10^{-1}$ & $8.8 \times 10^{-4}$ \\
\hline MWD-09 & 1.2 & $3.3 \times 10^{-2}$ & $5.3 \times 10^{-5}$ & 0.8 & $2.2 \times 10^{-2}$ & $3.5 \times 10^{-5}$ & 12 & $3.3 \times 10^{-1}$ & $5.3 \times 10^{-4}$ \\
\hline MWD-23 & 3.1 & $8.5 \times 10^{-2}$ & $1.4 \times 10^{-4}$ & - & - & - & 20 & $5.5 \times 10^{-1}$ & $8.8 \times 10^{-4}$ \\
\hline MWD-106 & 1.8 & $4.9 \times 10^{-2}$ & $7.9 \times 10^{-5}$ & - & - & - & 16 & $4.4 \times 10^{-1}$ & $7.0 \times 10^{-4}$ \\
\hline MWS-0S & - & - & - & - & - & - & 19 & $5.2 \times 10^{-1}$ & $8.3 \times 10^{-4}$ \\
\hline MWS-06 & 1 & $2.7 \times 10^{-2}$ & $4.4 \times 10^{-5}$ & - & - & - & 19 & $5.2 \times 10^{-1}$ & $8.3 \times 10^{-4}$ \\
\hline MWS-105 & 1.3 & $3.6 \times 10^{-2}$ & $5.7 \times 10^{-5}$ & - & - & - & 11 & $3.0 \times 10^{-1}$ & $4.8 \times 10^{-4}$ \\
\hline
\end{tabular}


TABLE 3.6 (Cont.)

\begin{tabular}{|c|c|c|c|c|c|c|c|c|c|}
\hline \multirow[b]{3}{*}{ Well ID } & \multicolumn{3}{|c|}{ Chloride } & \multicolumn{3}{|c|}{ Nitrate-N } & \multicolumn{3}{|c|}{ Sulfate } \\
\hline & \multirow[b]{2}{*}{$\begin{array}{c}\mathrm{EPC}^{\mathrm{a}} \\
(\mathrm{mg} / \mathrm{L})\end{array}$} & \multicolumn{2}{|c|}{ Intake (mg/kg-d) } & \multirow[b]{2}{*}{$\begin{array}{c}\mathrm{EPC}^{\mathrm{a}} \\
(\mathrm{mg} / \mathrm{L})\end{array}$} & \multicolumn{2}{|c|}{ Intake (mg/kg-d) } & \multirow[b]{2}{*}{$\begin{array}{c}\mathrm{EPC}^{\mathrm{a}} \\
(\mathrm{mg} / \mathrm{L})\end{array}$} & \multicolumn{2}{|c|}{ Intake (mg/kg-d) } \\
\hline & & Ingestion & Dermal & & Ingestion $^{b}$ & Dermal & & Ingestion & Dermal \\
\hline \multicolumn{10}{|c|}{ Unweathered (cont.) } \\
\hline MWS-106 & 1.6 & $4.4 \times 10^{-2}$ & $7.0 \times 10^{-5}$ & - & - & - & 11 & $3.0 \times 10^{-1}$ & $4.8 \times 10^{-4}$ \\
\hline MWS-109 & 1 & $2.7 \times 10^{-2}$ & $4.4 \times 10^{-5}$ & - & - & - & 12 & $3.3 \times 10^{-1}$ & $5.3 \times 10^{-4}$ \\
\hline TIL-4 & - & - & - & - & - & - & - & - & - \\
\hline USGS-1 & 3.7 & $1.0 \times 10^{-1}$ & $1.6 \times 10^{-4}$ & 0.79 & $2.2 \times 10^{-2}$ & $3.5 \times 10^{-5}$ & 15 & $4.1 \times 10^{-1}$ & $6.6 \times 10^{-4}$ \\
\hline USGS-6 & 1.8 & $4.9 \times 10^{-2}$ & $7.9 \times 10^{-5}$ & 0.55 & $1.5 \times 10^{-2}$ & $2.4 \times 10^{-5}$ & 15 & $4.1 \times 10^{-1}$ & $6.6 \times 10^{-4}$ \\
\hline \multicolumn{10}{|l|}{ Weathered } \\
\hline MW-2001 & 5.9 & $1.6 \times 10^{-1}$ & $2.6 \times 10^{-4}$ & 49 & 1.3 & $2.1 \times 10^{-3}$ & 12 & $3.3 \times 10^{-1}$ & $5.3 \times 10^{-4}$ \\
\hline$M W-2002$ & 6.6 & $1.8 \times 10^{-1}$ & $2.9 \times 10^{-4}$ & 130 & 3.6 & $5.7 \times 10^{-3}$ & 120 & 3.3 & $5.3 \times 10^{-3}$ \\
\hline$M W-2003$ & 9 & $2.5 \times 10^{-1}$ & $3.9 \times 10^{-4}$ & 310 & 8.5 & $1.4 \times 10^{-2}$ & 100 & 2.7 & $4.4 \times 10^{-3}$ \\
\hline MW-2004 & - & - & - & - & - & - & - & - & - \\
\hline MW-2005 & 3.5 & $9.6 \times 10^{-2}$ & $1.5 \times 10^{-4}$ & 66 & 1.8 & $2.9 \times 10^{-3}$ & 29 & $7.9 \times 10^{-1}$ & $1.3 \times 10^{-3}$ \\
\hline MW-2006 & 3.7 & $1.0 \times 10^{-1}$ & $1.6 \times 10^{-4}$ & 4.9 & $1.3 \times 10^{-1}$ & $2.1 \times 10^{-4}$ & 9.2 & $2.5 \times 10^{-1}$ & $4.0 \times 10^{-4}$ \\
\hline MW-2007 & 1.2 & $3.3 \times 10^{-2}$ & $5.3 \times 10^{-5}$ & 2.9 & $7.9 \times 10^{-2}$ & $1.3 \times 10^{-4}$ & 15 & $4.1 \times 10^{-1}$ & $6.6 \times 10^{-4}$ \\
\hline$M W-2008$ & - & - & - & - & - & - & - & - & - \\
\hline MW-2009 & - & - & - & - & - & - & - & - & - \\
\hline MW-2010 & 47 & 1.3 & $2.1 \times 10^{-3}$ & 1.4 & $3.8 \times 10^{-2}$ & $6.1 \times 10^{-5}$ & 41 & 1.1 & $1.8 \times 10^{-3}$ \\
\hline MW-2011 & 4.2 & $1.2 \times 10^{-1}$ & $1.8 \times 10^{-4}$ & 4.8 & $1.3 \times 10^{-1}$ & $2.1 \times 10^{-4}$ & 13 & $3.6 \times 10^{-1}$ & $5.7 \times 10^{-4}$ \\
\hline MW-2012 & 48 & 1.3 & $2.1 \times 10^{-3}$ & 0.53 & $1.5 \times 10^{-2}$ & $2.3 \times 10^{-5}$ & 58 & 1.6 & $2.5 \times 10^{-3}$ \\
\hline MW-2013 & 5.3 & $1.5 \times 10^{-1}$ & $2.3 \times 10^{-4}$ & 1 & $2.7 \times 10^{-2}$ & $4.4 \times 10^{-5}$ & 27 & $7.4 \times 10^{-1}$ & $1.2 \times 10^{-3}$ \\
\hline MW-2014 & 26 & $7.1 \times 10^{-1}$ & $1.1 \times 10^{-3}$ & 1.8 & $4.9 \times 10^{-2}$ & $7.9 \times 10^{-5}$ & 38 & 1.0 & $1.7 \times 10^{-3}$ \\
\hline MW-2015 & 1.1 & $2.9 \times 10^{-2}$ & $4.7 \times 10^{-5}$ & 0.53 & $1.5 \times 10^{-2}$ & $2.3 \times 10^{-5}$ & 132 & 3.6 & $5.8 \times 10^{-3}$ \\
\hline MW-2016 & - & - & - & - & - & - & - & - & - \\
\hline$M W-2017$ & 15 & $4.1 \times 10^{-1}$ & $6.6 \times 10^{-4}$ & 5.5 & $1.5 \times 10^{-1}$ & $2.4 \times 10^{-4}$ & 1,100 & 31 & $4.9 \times 10^{-2}$ \\
\hline MW-2018 & 7.9 & $2.2 \times 10^{-1}$ & $3.5 \times 10^{-4}$ & 0.67 & $1.8 \times 10^{-2}$ & $2.9 \times 10^{-5}$ & 11 & $3.0 \times 10^{-1}$ & $4.8 \times 10^{-4}$ \\
\hline$M W-2020$ & - & - & - & - & - & - & - & - & - \\
\hline MW-2030 & 24 & $6.5 \times 10^{-1}$ & $1.0 \times 10^{-3}$ & 1.3 & $3.6 \times 10^{-2}$ & $5.7 \times 10^{-5}$ & 50 & 1.4 & $2.2 \times 10^{-3}$ \\
\hline MW-2034 & 26 & $7.1 \times 10^{-1}$ & $1.1 \times 10^{-3}$ & 4.8 & $1.3 \times 10^{-1}$ & $2.1 \times 10^{-4}$ & 320 & 8.8 & $1.4 \times 10^{-2}$ \\
\hline MW-2035 & 1.1 & $2.9 \times 10^{-2}$ & $4.6 \times 10^{-5}$ & 0.63 & $1.7 \times 10^{-2}$ & $2.8 \times 10^{-5}$ & 1.9 & $5.1 \times 10^{-2}$ & $8.1 \times 10^{-5}$ \\
\hline MW-2036 & 1.2 & $3.4 \times 10^{-2}$ & $5.4 \times 10^{-5}$ & 4 & $1.1 \times 10^{-1}$ & $1.8 \times 10^{-4}$ & 3.8 & $1.0 \times 10^{-1}$ & $1.7 \times 10^{-4}$ \\
\hline
\end{tabular}


TABLE 3.6 (Cont.)

\begin{tabular}{|c|c|c|c|c|c|c|c|c|c|}
\hline \multirow[b]{3}{*}{ Well ID } & \multicolumn{3}{|c|}{ Chloride } & \multicolumn{3}{|c|}{ Nitrate-N } & \multicolumn{3}{|c|}{ Sulfate } \\
\hline & \multirow{2}{*}{$\begin{array}{c}\mathrm{EPC}^{\mathrm{a}} \\
(\mathrm{mg} / \mathrm{L})\end{array}$} & \multicolumn{2}{|c|}{ Intake (mg/kg-d) } & \multirow{2}{*}{$\begin{array}{c}\mathrm{EPC}^{\mathrm{a}} \\
(\mathrm{mg} / \mathrm{L})\end{array}$} & \multicolumn{2}{|c|}{ Intake (mg/kg-d) } & \multirow{2}{*}{$\begin{array}{c}\mathrm{EPC}^{\mathrm{a}} \\
(\mathrm{mg} / \mathrm{L})\end{array}$} & \multicolumn{2}{|c|}{ Intake (mg/kg-d) } \\
\hline & & Ingestion & Dermal & & Ingestion $^{b}$ & Dermal & & Ingestion & Dermal \\
\hline \multicolumn{10}{|c|}{ Weathered (cont.) } \\
\hline MW-2037 & 32 & $8.8 \times 10^{-1}$ & $1.4 \times 10^{-3}$ & 290 & 7.9 & $1.3 \times 10^{-2}$ & 130 & 3.6 & $5.7 \times 10^{-3}$ \\
\hline$M W-2038$ & - & - & - & 900 & $2.5 \times 10^{1}$ & $3.9 \times 10^{-2}$ & 110 & 3.0 & $4.8 \times 10^{-3}$ \\
\hline MW-2039 & 49 & 1.3 & $2.1 \times 10^{-3}$ & 52 & 1.4 & $2.3 \times 10^{-3}$ & 33 & $9.0 \times 10^{-1}$ & $1.4 \times 10^{-3}$ \\
\hline MW-2040 & 4 & $1.1 \times 10^{-1}$ & $1.8 \times 10^{-4}$ & 230 & 6.3 & $1.0 \times 10^{-2}$ & 14 & $3.8 \times 10^{-1}$ & $6.1 \times 10^{-4}$ \\
\hline MW-2041 & 7.7 & $2.1 \times 10^{-1}$ & $3.4 \times 10^{-4}$ & 300 & 8.2 & $1.3 \times 10^{-2}$ & 37 & 1.0 & $1.6 \times 10^{-3}$ \\
\hline MW-2042 & 8.8 & $2.4 \times 10^{-1}$ & $3.9 \times 10^{-4}$ & 5.6 & $1.5 \times 10^{-1}$ & $2.5 \times 10^{-4}$ & 24 & $6.5 \times 10^{-1}$ & $1.0 \times 10^{-3}$ \\
\hline MW-2043 & 4.2 & $1.2 \times 10^{-1}$ & $1.8 \times 10^{-4}$ & 5.8 & $1.6 \times 10^{-1}$ & $2.5 \times 10^{-4}$ & 15 & $4.1 \times 10^{-1}$ & $6.6 \times 10^{-4}$ \\
\hline MW-2044 & 19 & $5.2 \times 10^{-1}$ & $8.3 \times 10^{-4}$ & 1.3 & $3.6 \times 10^{-2}$ & $5.7 \times 10^{-5}$ & 130 & 3.6 & $5.7 \times 10^{-3}$ \\
\hline MW-3003 & 12 & $3.3 \times 10^{-1}$ & $5.3 \times 10^{-4}$ & 300 & 8.2 & $1.3 \times 10^{-2}$ & 140 & 3.7 & $5.9 \times 10^{-3}$ \\
\hline MW-3007 & - & - & - & - & - & - & - & - & - \\
\hline$M W-3008$ & - & - & - & - & - & - & - & - & - \\
\hline MW-3009 & - & - & - & - & - & - & - & - & - \\
\hline$M W-3010$ & - & - & - & - & - & - & - & - & - \\
\hline MW-3019 & - & - & - & 1.2 & $3.3 \times 10^{-2}$ & $5.3 \times 10^{-5}$ & - & - & - \\
\hline MW-3023 & 9.6 & $2.6 \times 10^{-1}$ & $4.2 \times 10^{-4}$ & 210 & 5.8 & $9.2 \times 10^{-3}$ & 250 & 6.8 & $1.1 \times 10^{-2}$ \\
\hline MW-3025 & 11 & $3.0 \times 10^{-1}$ & $4.8 \times 10^{-4}$ & 520 & $1.4 \times 10^{1}$ & $2.3 \times 10^{-2}$ & 55 & 1.5 & $2.4 \times 10^{-3}$ \\
\hline MW-3027 & 2.4 & $6.6 \times 10^{-2}$ & $1.1 \times 10^{-4}$ & 62 & 1.7 & $2.7 \times 10^{-3}$ & - & - & - \\
\hline$M W-4001$ & 3.1 & $8.5 \times 10^{-2}$ & $1.4 \times 10^{-4}$ & 40 & 1.1 & $1.8 \times 10^{-3}$ & 65 & 1.8 & $2.8 \times 10^{-3}$ \\
\hline$M W-4002$ & 0.99 & $2.7 \times 10^{-2}$ & $4.3 \times 10^{-5}$ & 5.2 & $1.4 \times 10^{-1}$ & $2.3 \times 10^{-4}$ & 14 & $3.8 \times 10^{-1}$ & $6.1 \times 10^{-4}$ \\
\hline MW-4003 & 4.8 & $1.3 \times 10^{-1}$ & $2.1 \times 10^{-4}$ & 0.65 & $1.8 \times 10^{-2}$ & $2.8 \times 10^{-5}$ & 27 & $7.4 \times 10^{-1}$ & $1.2 \times 10^{-3}$ \\
\hline MW-4005 & 5.7 & $1.6 \times 10^{-1}$ & $2.5 \times 10^{-4}$ & 1.6 & $4.4 \times 10^{-2}$ & $7.0 \times 10^{-5}$ & 19 & $5.2 \times 10^{-1}$ & $8.3 \times 10^{-4}$ \\
\hline MW-4006 & 1.6 & $4.4 \times 10^{-2}$ & $7.0 \times 10^{-5}$ & 14 & $3.8 \times 10^{-1}$ & $6.1 \times 10^{-4}$ & 24 & $6.6 \times 10^{-1}$ & $1.1 \times 10^{-3}$ \\
\hline MW-4010 & 1.1 & $3.0 \times 10^{-2}$ & $4.8 \times 10^{-5}$ & - & - & - & 23 & $6.3 \times 10^{-1}$ & $1.0 \times 10^{-3}$ \\
\hline$M W-4013$ & 7.6 & $2.1 \times 10^{-1}$ & $3.3 \times 10^{-4}$ & 94 & 2.6 & $4.1 \times 10^{-3}$ & 56 & 1.5 & $2.5 \times 10^{-3}$ \\
\hline$M W-4014$ & 1.7 & $4.7 \times 10^{-2}$ & $7.5 \times 10^{-5}$ & 5.8 & $1.6 \times 10^{-1}$ & $2.5 \times 10^{-4}$ & 25 & $6.8 \times 10^{-1}$ & $1.1 \times 10^{-3}$ \\
\hline MW-4015 & 8.1 & $2.2 \times 10^{-1}$ & $3.6 \times 10^{-4}$ & 4.2 & $1.2 \times 10^{-1}$ & $1.8 \times 10^{-4}$ & 27 & $7.4 \times 10^{-1}$ & $1.2 \times 10^{-3}$ \\
\hline MW-4016 & 0.81 & $2.2 \times 10^{-2}$ & $3.6 \times 10^{-5}$ & 0.04 & $1.1 \times 10^{-3}$ & $1.8 \times 10^{-6}$ & 14 & $3.8 \times 10^{-1}$ & $6.1 \times 10^{-4}$ \\
\hline$M W-4017$ & - & - & - & - & - & - & - & - & - \\
\hline$M W-4018$ & - & - & - & 2.7 & $7.4 \times 10^{-2}$ & $1.2 \times 10^{-4}$ & - & - & - \\
\hline MW-4019 & - & - & - & 0.26 & $7.1 \times 10^{-3}$ & $1.1 \times 10^{-5}$ & - & - & - \\
\hline
\end{tabular}


TABLE 3.6 (Cont.)

\begin{tabular}{|c|c|c|c|c|c|c|c|c|c|}
\hline \multirow[b]{3}{*}{ Well ID } & \multicolumn{3}{|c|}{ Chloride } & \multicolumn{3}{|c|}{ Nitrate-N } & \multicolumn{3}{|c|}{ Sulfate } \\
\hline & \multirow{2}{*}{$\begin{array}{c}\mathrm{EPC}^{\mathrm{a}} \\
(\mathrm{mg} / \mathrm{L})\end{array}$} & \multicolumn{2}{|c|}{ Intake (mg/kg-d) } & \multirow{2}{*}{$\begin{array}{r}\mathrm{EPC}^{\mathrm{a}} \\
(\mathrm{mg} / \mathrm{L}) \\
\end{array}$} & \multicolumn{2}{|c|}{ Intake (mg/kg-d) } & \multirow{2}{*}{$\begin{array}{c}\mathrm{EPC} \\
(\mathrm{mg} / \mathrm{L})\end{array}$} & \multicolumn{2}{|c|}{ Intake $(\mathrm{mg} / \mathrm{kg}-\mathrm{d})$} \\
\hline & & Ingestion & Dermal & & Ingestion $^{b}$ & Dermal & & Ingestion & Dermal \\
\hline \multicolumn{10}{|c|}{ Weathered (cont.) } \\
\hline MW-4020 & - & - & - & - & - & - & - & - & - \\
\hline$M W-4021$ & - & - & - & - & - & - & - & - & - \\
\hline $\mathrm{MW}-4023$ & - & - & - & 2.6 & $7.1 \times 10^{-2}$ & $1.1 \times 10^{-4}$ & - & - & - \\
\hline MW-4024 & 7.6 & $2.1 \times 10^{-1}$ & $3.3 \times 10^{-4}$ & 1.4 & $3.8 \times 10^{-2}$ & $6.5 \times 10^{-5}$ & 680 & 19 & $3.0 \times 10^{-2}$ \\
\hline MW-4025 & 8.7 & $2.4 \times 10^{-1}$ & $3.8 \times 10^{-4}$ & 1.1 & $3.0 \times 10^{-2}$ & $4.8 \times 10^{-5}$ & 26 & $7.1 \times 10^{-1}$ & $1.1 \times 10^{-3}$ \\
\hline MWD-15 & 1.4 & $3.8 \times 10^{-2}$ & $6.1 \times 10^{-5}$ & 4.3 & $1.2 \times 10^{-1}$ & $1.9 \times 10^{-4}$ & - & - & - \\
\hline MWD-25 & 11 & $3.0 \times 10^{-1}$ & $4.8 \times 10^{-4}$ & 0.38 & $1.0 \times 10^{-2}$ & $1.7 \times 10^{-5}$ & 45 & 1.2 & $2.0 \times 10^{-3}$ \\
\hline MWD-107 & 3.1 & $8.5 \times 10^{-2}$ & $1.4 \times 10^{-4}$ & - & - & - & 25 & $6.8 \times 10^{-1}$ & $1.1 \times 10^{-3}$ \\
\hline MWD-112 & 1.3 & $3.6 \times 10^{-2}$ & $5.7 \times 10^{-5}$ & - & - & - & 19 & $5.2 \times 10^{-1}$ & $8.3 \times 10^{-4}$ \\
\hline MWS-01 & 2.3 & $6.3 \times 10^{-2}$ & $1.0 \times 10^{-4}$ & 2.5 & $6.8 \times 10^{-2}$ & $1.1 \times 10^{-4}$ & - & - & - \\
\hline MWS-02 & 1 & $2.7 \times 10^{-2}$ & $4.4 \times 10^{-5}$ & 0.1 & $2.7 \times 10^{-3}$ & $4.4 \times 10^{-6}$ & 15 & $4.1 \times 10^{-1}$ & $6.6 \times 10^{-4}$ \\
\hline MWS-03 & 1.3 & $3.6 \times 10^{-2}$ & $5.7 \times 10^{-5}$ & - & - & - & 23 & $6.3 \times 10^{-1}$ & $1.0 \times 10^{-3}$ \\
\hline MWS-04 & 2.2 & $6.0 \times 10^{-2}$ & $9.6 \times 10^{-5}$ & 8.9 & $2.4 \times 10^{-1}$ & $3.9 \times 10^{-4}$ & 35 & $9.6 \times 10^{-1}$ & $1.5 \times 10^{-3}$ \\
\hline MWS-07 & 1.9 & $5.2 \times 10^{-2}$ & $8.3 \times 10^{-5}$ & 2.3 & $6.3 \times 10^{-2}$ & $1.0 \times 10^{-4}$ & 39 & 1.1 & $1.7 \times 10^{-3}$ \\
\hline MWS-08 & 3.9 & $1.1 \times 10^{-1}$ & $1.7 \times 10^{-4}$ & 1.9 & $5.2 \times 10^{-2}$ & $8.3 \times 10^{-5}$ & 33 & $9.0 \times 10^{-1}$ & $1.4 \times 10^{-3}$ \\
\hline MWS-09 & 0.96 & $2.6 \times 10^{-2}$ & $4.2 \times 10^{-5}$ & - & - & - & 12 & $3.3 \times 10^{-1}$ & $5.3 \times 10^{-4}$ \\
\hline MWS-10 & 2.1 & $5.8 \times 10^{-2}$ & $9.2 \times 10^{-5}$ & 8.6 & $2.4 \times 10^{-1}$ & $3.8 \times 10^{-4}$ & 64 & 1.8 & $2.8 \times 10^{-3}$ \\
\hline MWS-11 & 3.2 & $8.8 \times 10^{-2}$ & $1.4 \times 10^{-4}$ & 8.8 & $2.4 \times 10^{-1}$ & $3.9 \times 10^{-4}$ & 43 & 1.2 & $1.9 \times 10^{-3}$ \\
\hline MWS-12 & 1.9 & $5.2 \times 10^{-2}$ & $8.3 \times 10^{-5}$ & 2.9 & $7.9 \times 10^{-2}$ & $1.3 \times 10^{-4}$ & - & - & - \\
\hline MWS-13 & 6.6 & $1.8 \times 10^{-1}$ & $2.9 \times 10^{-4}$ & 1.2 & $3.3 \times 10^{-2}$ & $5.3 \times 10^{-5}$ & 600 & 16 & $2.6 \times 10^{-2}$ \\
\hline MWS-14 & 11 & $3.0 \times 10^{-1}$ & $4.8 \times 10^{-4}$ & 0.18 & $4.9 \times 10^{-3}$ & $7.9 \times 10^{-6}$ & 24 & $6.6 \times 10^{-1}$ & $1.1 \times 10^{-3}$ \\
\hline MWS-15 & 1.8 & $4.9 \times 10^{-2}$ & $7.9 \times 10^{-5}$ & 0.91 & $2.5 \times 10^{-2}$ & $4.0 \times 10^{-5}$ & 33 & $9.0 \times 10^{-1}$ & $1.4 \times 10^{-3}$ \\
\hline MWS-16 & 9 & $2.5 \times 10^{-1}$ & $3.9 \times 10^{-4}$ & 7.7 & $2.1 \times 10^{-1}$ & $3.4 \times 10^{-4}$ & 23 & $6.3 \times 10^{-1}$ & $1.0 \times 10^{-3}$ \\
\hline MWS-17 & 4.3 & $1.2 \times 10^{-1}$ & $1.9 \times 10^{-4}$ & 3.1 & $8.5 \times 10^{-2}$ & $1.4 \times 10^{-4}$ & 45 & 1.2 & $2.0 \times 10^{-3}$ \\
\hline MWS-19 & 1.4 & $3.8 \times 10^{-2}$ & $6.1 \times 10^{-5}$ & 0.15 & $4.1 \times 10^{-3}$ & $6.6 \times 10^{-6}$ & 20 & $5.5 \times 10^{-1}$ & $8.8 \times 10^{-4}$ \\
\hline MWS-20 & 2.4 & $6.6 \times 10^{-2}$ & $1.1 \times 10^{-4}$ & 5.6 & $1.5 \times 10^{-1}$ & $2.5 \times 10^{-4}$ & 17 & $4.7 \times 10^{-1}$ & $7.5 \times 10^{-4}$ \\
\hline MWS-21 & 26 & $7.1 \times 10^{-1}$ & $1.1 \times 10^{-3}$ & 520 & $1.4 \times 10^{1}$ & $2.3 \times 10^{-2}$ & 95 & 2.6 & $4.2 \times 10^{-3}$ \\
\hline MWS-22 & 4.3 & $1.2 \times 10^{-1}$ & $1.9 \times 10^{-4}$ & 3 & $8.2 \times 10^{-2}$ & $1.3 \times 10^{-4}$ & 18 & $4.9 \times 10^{-1}$ & $7.9 \times 10^{-4}$ \\
\hline MWS-24 & - & - & - & - & - & - & - & - & - \\
\hline MWS-25 & 6.8 & $1.9 \times 10^{-1}$ & $3.0 \times 10^{-4}$ & 0.6 & $1.6 \times 10^{-2}$ & $2.6 \times 10^{-5}$ & 37 & 1.0 & $1.6 \times 10^{-3}$ \\
\hline
\end{tabular}


TABLE 3.6 (Cont.)

\begin{tabular}{|c|c|c|c|c|c|c|c|c|c|}
\hline \multirow[b]{3}{*}{ Well ID } & \multicolumn{3}{|c|}{ Chloride } & \multicolumn{3}{|c|}{ Nitrate-N } & \multicolumn{3}{|c|}{ Sulfate } \\
\hline & \multirow[b]{2}{*}{$\begin{array}{c}\mathrm{EPC}^{\mathrm{a}} \\
(\mathrm{mg} / \mathrm{L})\end{array}$} & \multicolumn{2}{|c|}{ Intake (mg/kg-d) } & \multirow[b]{2}{*}{$\begin{array}{c}\mathrm{EPC}^{\mathrm{a}} \\
(\mathrm{mg} / \mathrm{L})\end{array}$} & \multicolumn{2}{|c|}{ Intake (mg/kg-d) } & \multirow[b]{2}{*}{$\begin{array}{c}\mathrm{EPC}^{\mathrm{a}} \\
(\mathrm{mg} / \mathrm{L})\end{array}$} & \multicolumn{2}{|c|}{ Intake (mg/kg-d) } \\
\hline & & Ingestion & Dermal & & Ingestion $^{b}$ & Dermal & & Ingestion & Dermal \\
\hline \multicolumn{10}{|c|}{ Weathered (cont.) } \\
\hline MWS-26 & - & - & - & 0.52 & $1.4 \times 10^{-2}$ & $2.3 \times 10^{-5}$ & - & - & - \\
\hline MWS-104 & - & - & - & - & - & - & 11 & $3.0 \times 10^{-1}$ & $4.8 \times 10^{-4}$ \\
\hline MWS-107 & 3.9 & $1.1 \times 10^{-1}$ & $1.7 \times 10^{-4}$ & 1.6 & $4.4 \times 10^{-2}$ & $7.0 \times 10^{-5}$ & 16 & $4.4 \times 10^{-1}$ & $7.0 \times 10^{-4}$ \\
\hline MWS-110 & 1.6 & $4.4 \times 10^{-2}$ & $7.0 \times 10^{-5}$ & 0.8 & $2.2 \times 10^{-2}$ & $3.5 \times 10^{-5}$ & 20 & $5.5 \times 10^{-1}$ & $8.8 \times 10^{-4}$ \\
\hline MWS-112 & 3.1 & $8.5 \times 10^{-2}$ & $1.4 \times 10^{-4}$ & 0.13 & $3.6 \times 10^{-3}$ & $5.7 \times 10^{-6}$ & 20 & $5.5 \times 10^{-1}$ & $8.8 \times 10^{-4}$ \\
\hline USGS-2 & 1.2 & $3.3 \times 10^{-2}$ & $5.3 \times 10^{-5}$ & - & - & - & 10 & $2.7 \times 10^{-1}$ & $4.4 \times 10^{-4}$ \\
\hline USGS-3 & 2.2 & $6.0 \times 10^{-2}$ & $9.6 \times 10^{-5}$ & 0.88 & $2.4 \times 10^{-2}$ & $3.9 \times 10^{-5}$ & 17 & $4.7 \times 10^{-1}$ & $7.5 \times 10^{-4}$ \\
\hline USGS-4 & 2.8 & $7.7 \times 10^{-2}$ & $1.2 \times 10^{-4}$ & 1.5 & $4.1 \times 10^{-2}$ & $6.6 \times 10^{-5}$ & 25 & $6.8 \times 10^{-1}$ & $1.1 \times 10^{-3}$ \\
\hline USGS-5 & 1.2 & $3.3 \times 10^{-2}$ & $5.3 \times 10^{-5}$ & 0.23 & $6.3 \times 10^{-3}$ & $1.0 \times 10^{-5}$ & 8.1 & $2.2 \times 10^{-1}$ & $3.6 \times 10^{-4}$ \\
\hline USGS-7 & - & - & - & - & - & - & - & - & - \\
\hline USGS-8 & 2.9 & $7.9 \times 10^{-2}$ & $1.3 \times 10^{-4}$ & 3.2 & $8.8 \times 10^{-2}$ & $1.4 \times 10^{-4}$ & 13 & $3.6 \times 10^{-1}$ & $5.7 \times 10^{-4}$ \\
\hline USGS-9 & 5.1 & $1.4 \times 10^{-1}$ & $2.2 \times 10^{-4}$ & 3.2 & $8.8 \times 10^{-2}$ & $1.4 \times 10^{-4}$ & 19 & $5.2 \times 10^{-1}$ & $8.3 \times 10^{-4}$ \\
\hline
\end{tabular}

a $\mathrm{EPC}=$ exposure point concentration, which is the maximum uranium value for each spring from the 1995 joint DOE/DA sampling rounds.

b Intakes of nitrate by an infant can be calculated by multiplying the EPC by 0.16 . Intakes for ingestion of nitrate by an infant range from $8.0 \times 10^{-4}(\mathrm{MW}-2021)$ to $140(\mathrm{MW}-2038)$.

c A hyphen (-) indicates the parameter was not detected. 
TABLE 3.7 Estimated Noncarcinogenic Intakes of Organic Compound COPCs for the Hypothetical Future Resident

\begin{tabular}{|c|c|c|c|c|c|c|c|c|c|c|c|c|}
\hline \multirow[b]{3}{*}{ Well ID } & \multicolumn{3}{|c|}{$1,3,5$-TNB } & \multicolumn{3}{|c|}{ 1,3-DNB } & \multicolumn{3}{|c|}{ 2,4,6-TNT } & \multicolumn{3}{|c|}{ 2,4-DNT } \\
\hline & \multirow[b]{2}{*}{$\begin{array}{c}\mathrm{EPC}^{\mathrm{a}} \\
(\mu \mathrm{g} / \mathrm{L})\end{array}$} & \multicolumn{2}{|c|}{ Intake $(\mathrm{mg} / \mathrm{kg}-\mathrm{d})$} & \multirow[b]{2}{*}{$\begin{array}{c}\mathrm{EPC}^{\mathrm{a}} \\
(\mu \mathrm{g} / \mathrm{L})\end{array}$} & \multicolumn{2}{|c|}{ Intake (Ing/kg-d) } & \multirow[b]{2}{*}{$\begin{array}{c}\mathrm{EPC}^{\mathrm{a}} \\
(\mu \mathrm{g} / \mathrm{L})\end{array}$} & \multicolumn{2}{|c|}{ Intake (mg/kg-d) } & \multirow[b]{2}{*}{$\begin{array}{c}\mathrm{EPC}^{\mathrm{a}} \\
(\mu \mathrm{g} / \mathrm{L})\end{array}$} & \multicolumn{2}{|c|}{ Intake (mg/kg-d) } \\
\hline & & Ingestion & Dermal & & Ingestion & Dermal & & Ingestion & Dermal & & Ingestion & Dermal \\
\hline \multicolumn{13}{|l|}{ Deep Wells } \\
\hline MWD-05 & $-b$ & - & - & - & - & - & - & - & - & - & - & - \\
\hline MWD-18 & - & - & - & - & - & - & - & - & - & - & - & $\dot{-}$ \\
\hline MWGS-01 & - & - & - & - & - & - & - & - & - & - & - & - \\
\hline MWGS-02 & - & - & - & - & - & - & - & - & - & - & - & - \\
\hline MWS-18 & - & - & - & - & - & - & - & - & - & - & - & - \\
\hline MWS-101 & - & - & - & - & - & - & - & - & - & - & - & - \\
\hline MWS-102 & - & - & - & - & - & - & - & - & - & - & - & - \\
\hline MWS-103 & - & - & - & - & - & - & - & - & - & - & - & - \\
\hline TIL-3 & - & - & - & - & - & - & - & - & - & - & - & - \\
\hline \multicolumn{13}{|l|}{ Overburden } \\
\hline MW-2031 & - & - & - & - & - & - & - & - & - & - & - & - \\
\hline MW-2032 & 4.9 & $1.3 \times 10^{-4}$ & $2.1 \times 10^{-7}$ & - & - & - & 6.7 & $1.8 \times 10^{-4}$ & $2.9 \times 10^{-7}$ & 0.14 & $3.8 \times 10^{-6}$ & $6.1 \times 10^{-9}$ \\
\hline MW-2033 & 4.5 & $1.2 \times 10^{-4}$ & $2.0 \times 10^{-7}$ & - & - & - & 1.2 & $3.3 \times 10^{-5}$ & $5.3 \times 10^{-8}$ & 0.55 & $1.5 \times 10^{-5}$ & $2.4 \times 10^{-8}$ \\
\hline MW-3001 & - & - & - & - & - & - & - & - & - & - & - & - \\
\hline MW-3013 & - & - & - & - & - & - & - & - & - & - & - & - \\
\hline MW-3018 & - & - & - & - & - & - & - & - & - & - & - & - \\
\hline MW-3022 & - & - & - & - & - & - & - & - & - & - & - & - \\
\hline USGS-2A & - & - & - & - & - & - & - & - & - & - & - & - \\
\hline$M W V-01$ & 0.038 & $1.0 \times 10^{-6}$ & $1.7 \times 10^{-9}$ & - & - & - & 11 & $3.0 \times 10^{-4}$ & $4.8 \times 10^{-7}$ & 0.11 & $3.0 \times 10^{-6}$ & $4.8 \times 10^{-9}$ \\
\hline MWV-02 & - & - & - & - & - & - & 0.11 & $3.0 \times 10^{-6}$ & $4.8 \times 10^{-9}$ & 0.059 & $1.6 \times 10^{-6}$ & $2.6 \times 10^{-9}$ \\
\hline MWV-09 & 14 & $3.8 \times 10^{-4}$ & $6.1 \times 10^{-7}$ & 0.40 & $1.1 \times 10^{-5}$ & $1.8 \times 10^{-8}$ & 30 & $8.2 \times 10^{-4}$ & $1.3 \times 10^{-6}$ & 20 & $5.5 \times 10^{-4}$ & $8.8 \times 10^{-7}$ \\
\hline MWV-13 & - & - & - & - & - & - & - & - & - & - & - & - \\
\hline MWV-16 & 0.33 & $9.0 \times 10^{-6}$ & $1.4 \times 10^{-8}$ & - & - & - & 0.27 & $7.4 \times 10^{-6}$ & $1.2 \times 10^{-8}$ & - & - & - \\
\hline MWV-17 & - & - & - & - & - & - & - & - & - & - & - & - \\
\hline$M W V-18$ & - & - & - & - & - & - & - & - & - & - & - & - \\
\hline MWV-22 & - & - & - & - & - & - & - & - & - & - & - & -7 \\
\hline$M W V-24 R$ & 3.1 & $8.5 \times 10^{-5}$ & $1.4 \times 10^{-7}$ & - & - & - & 1.1 & $3.0 \times 10^{-5}$ & $4.8 \times 10^{-8}$ & 0.13 & $3.6 \times 10^{-6}$ & $5.7 \times 10^{-9}$ \\
\hline
\end{tabular}


TABLE 3.7 (Cont.)

\begin{tabular}{|c|c|c|c|c|c|c|c|c|c|c|c|c|}
\hline \multirow[b]{3}{*}{ Well ID } & \multicolumn{3}{|c|}{$1,3,5$-TNB } & \multicolumn{3}{|c|}{ 1,3-DNB } & \multicolumn{3}{|c|}{$2,4,6-\mathrm{TNT}$} & \multicolumn{3}{|c|}{ 2,4-DNT } \\
\hline & \multirow[b]{2}{*}{$\begin{array}{c}\mathrm{EPC}^{\mathrm{a}} \\
(\mu \mathrm{g} / \mathrm{L})\end{array}$} & \multicolumn{2}{|c|}{ Intake (mg/kg-d) } & \multirow[b]{2}{*}{$\begin{array}{c}\mathrm{EPC}^{\mathrm{a}} \\
(\mu \mathrm{g} / \mathrm{L})\end{array}$} & \multicolumn{2}{|c|}{ Intake (mg/kg-d) } & \multirow[b]{2}{*}{$\begin{array}{c}\mathrm{EPC}^{\mathrm{a}} \\
(\mu \mathrm{g} / \mathrm{L})\end{array}$} & \multicolumn{2}{|c|}{ Intake (mg/kg-d) } & \multirow[b]{2}{*}{$\begin{array}{c}\mathrm{EPC}^{\mathrm{a}} \\
(\mu \mathrm{g} / \mathrm{L})\end{array}$} & \multicolumn{2}{|c|}{ Intake (mg/kg-d) } \\
\hline & & Ingestion & Dermal & & Ingestion & Dermal & & Ingestion & Dermal & & Ingestion & Dermal \\
\hline \multicolumn{13}{|l|}{ Unweathered } \\
\hline MW-2019 & - & - & - & - & - & - & - & - & - & - & - & - \\
\hline MW-2021 & - & - & - & - & - & - & - & - & - & - & - & - \\
\hline MW-2022 & - & - & - & - & - & - & - & - & - & - & - & - \\
\hline$M W-2023$ & - & - & - & - & - & - & - & - & - & - & - & - \\
\hline MW-2024 & - & - & - & - & - & - & - & - & - & - & - & - \\
\hline MW-2025 & - & - & - & - & - & - & - & - & - & - & - & - \\
\hline MW-2026 & - & - & - & - & - & - & - & - & - & - & - & - \\
\hline MW-2027 & - & - & - & - & - & - & - & - & - & - & - & - \\
\hline$M W-2028$ & - & - & - & - & - & - & - & - & - & - & - & - \\
\hline MW-2029 & - & - & - & - & - & - & - & - & - & - & - & - \\
\hline MW-3002 & - & - & - & - & - & - & - & - & - & - & - & - \\
\hline MW-3006 & - & - & - & - & - & - & - & - & - & - & - & - \\
\hline MW-3024 & - & - & - & - & - & - & - & - & - & 0.13 & $3.6 \times 10^{-6}$ & $5.7 \times 10^{-9}$ \\
\hline MW-3026 & 0.14 & $3.8 \times 10^{-6}$ & $6.1 \times 10^{-9}$ & - & - & - & - & - & - & 0.072 & $2.0 \times 10^{-6}$ & $3.2 \times 10^{-9}$ \\
\hline$M W-4004$ & - & - & - & - & - & - & - & - & - & - & - & - \\
\hline$M W-4007$ & - & - & - & - & - & - & - & - & - & - & - & - \\
\hline$M W-4008$ & - & - & - & - & - & - & - & - & - & - & - & - \\
\hline MW-4009 & - & - & - & - & - & - & - & - & - & - & - & - \\
\hline MW-4011 & - & - & - & - & - & - & - & - & - & - & - & - \\
\hline$M W-4012$ & - & - & - & - & - & - & - & - & - & - & - & - \\
\hline$M W-4022$ & - & - & - & - & - & - & - & - & - & - & - & - \\
\hline MWD-02 & - & - & - & - & - & - & - & - & - & - & - & - \\
\hline MWD-06 & - & - & - & - & - & - & - & - & - & - & - & - \\
\hline MWD-09 & 0.065 & $1.8 \times 10^{-6}$ & $2.8 \times 10^{-9}$ & - & - & - & - & - & - & 0.042 & $1.2 \times 10^{-6}$ & $1.8 \times 10^{-9}$ \\
\hline MWD-23 & - & - & - & - & - & - & - & - & - & - & - & - \\
\hline MWD-106 & - & - & - & - & - & - & - & - & - & - & - & - \\
\hline MWS-05 & - & - & - & - & - & - & - & - & - & - & - & - \\
\hline MWS-06 & - & - & - & - & - & - & - & - & - & - & - & - \\
\hline MWS-105 & - & - & - & - & - & - & - & - & - & - & - & - \\
\hline MWS-106 & - & - & - & - & - & - & - & - & - & - & - & - \\
\hline MWS-109 & - & - & - & - & - & - & - & - & - & - & - & - \\
\hline TIL-4 & - & - & - & - & - & - & - & - & - & - & - & \\
\hline USGS-I & 0.063 & $1.7 \times 10^{-6}$ & $2.8 \times 10^{-9}$ & 0.14 & $3.8 \times 10^{-6}$ & $6.1 \times 10^{-9}$ & - & - & - & 0.051 & $1.4 \times 10^{-6}$ & $2.2 \times 10^{-9}$ \\
\hline USGS-6 & - & - & - & - & - & - & - & - & - & - & - & - \\
\hline
\end{tabular}


TABLE 3.7 (Cont.)

\begin{tabular}{|c|c|c|c|c|c|c|c|c|c|c|c|c|}
\hline \multirow[b]{3}{*}{ Well ID } & \multicolumn{3}{|c|}{ 1,3,5-TNB } & \multicolumn{3}{|c|}{ 1,3-DNB } & \multicolumn{3}{|c|}{ 2,4,6-TNT } & \multicolumn{3}{|c|}{ 2,4-DNT } \\
\hline & \multirow{2}{*}{$\begin{array}{c}\mathrm{EPC}^{\mathrm{a}} \\
(\mu \mathrm{g} / \mathrm{L})\end{array}$} & \multicolumn{2}{|c|}{ Intake $(\mathrm{mg} / \mathrm{kg}-\mathrm{d})$} & \multirow{2}{*}{$\begin{array}{l}E^{E P C^{a}} \\
(\mu g / L)\end{array}$} & \multicolumn{2}{|c|}{ Intake $(\mathrm{mg} / \mathrm{kg}-\mathrm{d})$} & \multirow{2}{*}{$\begin{array}{c}\mathrm{EPC}^{a} \\
(\mu \mathrm{g} / \mathrm{L})\end{array}$} & \multicolumn{2}{|c|}{ Intake (mg/kg-d) } & \multirow{2}{*}{$\begin{array}{l}\mathrm{EPC}^{\mathrm{a}} \\
(\mu \mathrm{g} / \mathrm{L})\end{array}$} & \multicolumn{2}{|c|}{ Intake $(\mathrm{mg} / \mathrm{kg}-\mathrm{d})$} \\
\hline & & Ingestion & Dermal & & Ingestion & Dermal & & Ingestion & Dermal & & Ingestion & Dermal \\
\hline \multicolumn{13}{|l|}{ Weathered } \\
\hline MW-200! & 0.054 & $1.5 \times 10^{-6}$ & $2.4 \times 10^{-9}$ & - & - & - & - & - & - & 0.13 & $3.6 \times 10^{-6}$ & $5.7 \times 10^{-9}$ \\
\hline MW-2002 & - & - & - & - & - & - & - & - & - & 0.070 & $1.9 \times 10^{-6}$ & $3.1 \times 10^{-9}$ \\
\hline$M W-2003$ & - & - & - & - & - & - & - & - & - & 0.15 & $4.1 \times 10^{-6}$ & $6.6 \times 10^{-9}$ \\
\hline MW-2004 & - & - & - & - & - & - & - & - & - & - & - & - \\
\hline MW-2005 & 0.035 & $9.6 \times 10^{-7}$ & $1.5 \times 10^{-9}$ & - & - & - & - & - & - & 0.061 & $1.7 \times 10^{-6}$ & $2.7 \times 10^{-9}$ \\
\hline MW-2006 & - & - & - & - & - & - & - & - & - & 0.14 & $3.8 \times 10^{-6}$ & $6.1 \times 10^{-9}$ \\
\hline MW-2007 & - & - & - & - & - & - & - & - & - & - & - & - \\
\hline MW-2008 & - & - & - & - & - & - & - & - & - & - & - & - \\
\hline MW-2009 & - & - & - & - & - & - & - & - & - & - & - & - \\
\hline MW-2010 & 0.15 & $4.1 \times 10^{-6}$ & $6.6 \times 10^{-9}$ & - & - & - & 0.34 & $9.3 \times 10^{-6}$ & $1.5 \times 10^{-8}$ & 0.094 & $2.6 \times 10^{-6}$ & $4.1 \times 10^{-9}$ \\
\hline MW-2011 & 0.4 & $1.1 \times 10^{-5}$ & $1.8 \times 10^{-8}$ & - & - & - & - & - & - & 0.20 & $5.5 \times 10^{-6}$ & $8.8 \times 10^{-9}$ \\
\hline MW-2012 & 1.4 & $3.8 \times 10^{-5}$ & $6.1 \times 10^{-8}$ & - & - & - & 0.46 & $1.3 \times 10^{-5}$ & $2.0 \times 10^{-8}$ & 0.099 & $2.7 \times 10^{-6}$ & $4.3 \times 10^{-9}$ \\
\hline$M W-2013$ & 6.2 & $1.7 \times 10^{-4}$ & $2.7 \times 10^{-7}$ & - & - & - & 0.85 & $2.3 \times 10^{-5}$ & $3.7 \times 10^{-8}$ & 0.36 & $9.9 \times 10^{-6}$ & $1.6 \times 10^{-8}$ \\
\hline$M W-2014$ & 1.9 & $5.2 \times 10^{-5}$ & $8.3 \times 10^{-8}$ & 0.86 & $2.4 \times 10^{-5}$ & $3.8 \times 10^{-8}$ & 0.044 & $1.2 \times 10^{-6}$ & $1.9 \times 10^{-9}$ & 0.16 & $4.4 \times 10^{-6}$ & $7.0 \times 10^{-9}$ \\
\hline MW-2015 & - & - & - & - & - & - & - & - & - & - & - & - \\
\hline MW-2016 & - & - & - & - & - & - & - & - & - & - & - & - \\
\hline$M W-2017$ & - & - & - & - & - & - & - & - & - & - & - & - \\
\hline MW-2018 & - & - & - & - & - & - & - & - & - & - & - & - \\
\hline MW-2020 & - & - & - & - & - & - & - & - & - & - & - & - \\
\hline MW-2030 & 8.3 & $2.3 \times 10^{-4}$ & $3.6 \times 10^{-7}$ & - & - & - & 29 & $7.9 \times 10^{-4}$ & $1.3 \times 10^{-6}$ & 0.25 & $6.8 \times 10^{-6}$ & $1.1 \times 10^{-8}$ \\
\hline$M W-2034$ & - & - & - & - & - & - & - & - & - & - & - & - \\
\hline MW-2035 & - & - & - & - & - & - & - & - & - & - & - & - \\
\hline MW-2036 & - & - & - & - & - & - & - & - & - & - & - & - \\
\hline MW-2037 & 0.19 & $5.2 \times 10^{-6}$ & $8.3 \times 10^{-9}$ & - & - & - & - & - & - & 0.56 & $1.5 \times 10^{-5}$ & $2.5 \times 10^{-8}$ \\
\hline MW-2038 & 0.24 & $6.6 \times 10^{-6}$ & $1.1 \times 10^{-8}$ & - & - & - & - & - & - & 1.7 & $4.7 \times 10^{-5}$ & $7.5 \times 10^{-8}$ \\
\hline$M W-2039$ & 7.3 & $2.0 \times 10^{-4}$ & $3.2 \times 10^{-7}$ & - & - & - & - & - & - & 0.12 & $3.3 \times 10^{-6}$ & $5.3 \times 10^{-9}$ \\
\hline MW-2040 & - & - & - & - & - & - & - & - & - & - & - & - \\
\hline$M W-2041$ & - & - & - & - & - & - & - & - & - & - & - & - \\
\hline $\mathrm{MW}-2042$ & - & - & - & - & - & - & - & - & - & - & - & - \\
\hline MW-2043 & - & - & - & - & - & - & - & - & - & 0.087 & $2.4 \times 10^{-6}$ & $3.8 \times 10^{-9}$ \\
\hline MW-2044 & - & - & - & - & - & - & - & - & - & - & - & - \\
\hline MW-3003 & - & - & - & - & - & - & - & - & - & 0.17 & $4.7 \times 10^{-6}$ & $7.5 \times 10^{-9}$ \\
\hline
\end{tabular}


TABLE 3.7 (Cont.)

\begin{tabular}{|c|c|c|c|c|c|c|c|c|c|c|c|c|}
\hline \multirow[b]{3}{*}{ Well ID } & \multicolumn{3}{|c|}{$1,3,5-\mathrm{TNB}$} & \multicolumn{3}{|c|}{ 1,3-DNB } & \multicolumn{3}{|c|}{ 2,4,6-TNT } & \multicolumn{3}{|c|}{ 2,4-DNT } \\
\hline & \multirow[b]{2}{*}{$\begin{array}{c}\mathrm{EPC}^{\mathrm{a}} \\
(\mu \mathrm{g} / \mathrm{L})\end{array}$} & \multicolumn{2}{|c|}{ Intake $(\mathrm{mg} / \mathrm{kg}-\mathrm{d})$} & \multirow[b]{2}{*}{$\begin{array}{c}\mathrm{EPC}^{\mathrm{a}} \\
(\mu \mathrm{g} / \mathrm{L})\end{array}$} & \multicolumn{2}{|c|}{ Intake $(\mathrm{mg} / \mathrm{kg}-\mathrm{d})$} & \multirow[b]{2}{*}{$\begin{array}{c}\mathrm{EPC}^{\mathrm{a}} \\
(\mu \mathrm{g} / \mathrm{L})\end{array}$} & \multicolumn{2}{|c|}{ Intake $(\mathrm{mg} / \mathrm{kg}-\mathrm{d})$} & \multirow[b]{2}{*}{$\begin{array}{c}\text { EPC }^{\mathrm{a}} \\
(\mu \mathrm{g} / \mathrm{L})\end{array}$} & \multicolumn{2}{|c|}{ Intake (mg/kg-d) } \\
\hline & & Ingestion & Dermal & & Ingestion & Dermal & & Ingestion & Dermal & & Ingestion & Dermal \\
\hline \multicolumn{13}{|c|}{ Weathered (cont.) } \\
\hline MW-3007 & - & - & - & - & - & - & - & - & - & - & - & - \\
\hline MW-3008 & - & - & - & - & - & - & - & - & - & - & - & - \\
\hline MW-3009 & - & - & - & - & - & - & - & - & - & - & - & - \\
\hline$M W-3010$ & - & - & - & - & - & - & - & - & - & - & - & - \\
\hline MW-3019 & - & - & - & - & - & - & - & - & - & - & - & - \\
\hline MW-3023 & - & - & - & - & - & - & - & - & - & 5 & $1.4 \times 10^{-4}$ & $2.2 \times 10^{-7}$ \\
\hline$M W-3025$ & - & - & - & - & - & - & - & - & - & 0.094 & $2.6 \times 10^{-6}$ & $4.1 \times 10^{-9}$ \\
\hline MW-3027 & 0.074 & $2.0 \times 10^{-6}$ & $3.2 \times 10^{-9}$ & - & - & - & - & - & - & 0.058 & $1.6 \times 10^{-6}$ & $2.5 \times 10^{-9}$ \\
\hline$M W-4001$ & 39 & $1.1 \times 10^{-3}$ & $1.7 \times 10^{-6}$ & - & - & - & 1.8 & $4.9 \times 10^{-5}$ & $7.9 \times 10^{-8}$ & 1.3 & $3.6 \times 10^{-5}$ & $5.7 \times 10^{-8}$ \\
\hline MW-4002 & 0,062 & $1.7 \times 10^{-6}$ & $2.7 \times 10^{-9}$ & - & - & - & 1.8 & $4.9 \times 10^{-5}$ & $7.9 \times 10^{-8}$ & 0.14 & $3.8 \times 10^{-6}$ & $6.1 \times 10^{-9}$ \\
\hline MW-4003 & - & - & - & - & - & - & - & - & - & - & - & - \\
\hline MW-4005 & - & - & - & - & - & - & - & - & - & - & - & - \\
\hline MW-4006 & 19 & $5.2 \times 10^{-4}$ & $8.3 \times 10^{-7}$ & - & - & - & - & - & - & 0.16 & $4.4 \times 10^{-6}$ & $7.0 \times 10^{-9}$ \\
\hline MW-4010 & - & - & - & - & - & - & - & - & - & - & - & - \\
\hline MW-4013 & 27 & $7.4 \times 10^{-4}$ & $1.2 \times 10^{-6}$ & - & - & - & 0.046 & $1.3 \times 10^{-6}$ & $2.0 \times 10^{-9}$ & 0.077 & $2.1 \times 10^{-6}$ & $3.4 \times 10^{-9}$ \\
\hline MW-4014 & 0.11 & $3.0 \times 10^{-6}$ & $4.8 \times 10^{-9}$ & - & - & - & - & - & - & 0.026 & $7.1 \times 10^{-7}$ & $1.1 \times 10^{-9}$ \\
\hline$M W-4015$ & 1.8 & $4.9 \times 10^{-5}$ & $7.9 \times 10^{-8}$ & - & - & - & - & - & - & 0.19 & $5.2 \times 10^{-6}$ & $8.3 \times 10^{-9}$ \\
\hline MW-4016 & - & - & - & - & - & - & - & - & - & - & - & - \\
\hline MW-4017 & - & - & - & - & - & - & - & - & - & - & - & - \\
\hline MW-4018 & - & - & - & - & - & - & - & - & - & - & - & - \\
\hline$M W-4019$ & - & - & - & - & - & - & - & - & - & - & - & - \\
\hline MW-4020 & - & - & - & - & - & - & - & - & - & - & - & - \\
\hline$M W-4021$ & - & - & - & - & - & - & - & - & - & - & - & - \\
\hline MW-4023 & 0.088 & $2.4 \times 10^{-6}$ & $3.9 \times 10^{-9}$ & - & - & - & - & - & - & 0.067 & $1.8 \times 10^{-6}$ & $2.9 \times 10^{-9}$ \\
\hline $\mathrm{MW}-4024$ & - & - & - & - & - & - & - & - & - & - & - & - \\
\hline MW-4025 & - & - & - & - & - & - & - & - & - & - & - & - \\
\hline MWD-15 & - & - & - & - & - & - & - & - & - & - & - & - \\
\hline MWD-25 & - & - & - & - & - & - & - & - & - & - & - & - \\
\hline MWD-107 & 0.047 & $1.3 \times 10^{-6}$ & $2.1 \times 10^{-9}$ & - & - & - & - & - & - & 0.02 & $5.5 \times 10^{-7}$ & $8.8 \times 10^{-10}$ \\
\hline MWD-112 & - & - & - & - & - & - & - & - & - & - & - & - \\
\hline MWS-0I & - & - & - & - & - & - & - & - & - & - & - & - \\
\hline MWS-02 & - & - & - & - & - & - & - & - & - & - & - & - \\
\hline MWS-03 & - & - & - & - & - & - & - & - & - & - & - & - \\
\hline
\end{tabular}


TABLE 3.7 (Cont.)

\begin{tabular}{|c|c|c|c|c|c|c|c|c|c|c|c|c|}
\hline \multirow[b]{3}{*}{ Well ID } & \multirow[b]{3}{*}{$\begin{array}{c}\mathrm{EPC}^{\mathrm{a}} \\
(\mu \mathrm{g} / \mathrm{L})\end{array}$} & \multicolumn{2}{|c|}{ 1,3,5-TNB } & \multicolumn{3}{|c|}{ 1,3-DNB } & \multicolumn{3}{|c|}{ 2,4,6-TNT } & \multicolumn{3}{|c|}{ 2,4-DNT } \\
\hline & & \multicolumn{2}{|c|}{ Intake (mg/kg-d) } & \multirow[b]{2}{*}{$\begin{array}{c}\mathrm{EPC}^{\mathrm{a}} \\
(\mu \mathrm{g} / \mathrm{L})\end{array}$} & \multicolumn{2}{|c|}{ Intake $(\mathrm{mg} / \mathrm{kg}-\mathrm{d})$} & \multirow[b]{2}{*}{$\begin{array}{l}\mathrm{EPC}^{\mathrm{a}} \\
(\mu \mathrm{g} / \mathrm{L})\end{array}$} & \multicolumn{2}{|c|}{ Intake $(\mathrm{mg} / \mathrm{kg} \cdot \mathrm{d})$} & \multirow[b]{2}{*}{$\begin{array}{c}\mathrm{EPC}^{\mathrm{a}} \\
(\mu \mathrm{g} / \mathrm{L})\end{array}$} & \multicolumn{2}{|c|}{ Intake $(\mathrm{mg} / \mathrm{kg}-\mathrm{d})$} \\
\hline & & Ingestion & Dermal & & Ingestion & Dermal & & Ingestion & Dermal & & Ingestion & Dermal \\
\hline \multicolumn{13}{|c|}{ Weathered (cont.) } \\
\hline MWS-04 & 11 & $3.0 \times 10^{-4}$ & $4.8 \times 10^{-7}$ & - & - & - & 1.2 & $3.3 \times 10^{-5}$ & $5.3 \times 10^{-8}$ & 0.1 & $2.7 \times 10^{-6}$ & $4.4 \times 10^{-9}$ \\
\hline MWS-07 & 18 & $4.9 \times 10^{-4}$ & $7.9 \times 10^{-7}$ & - & - & - & 2.6 & $7.1 \times 10^{-5}$ & $1.1 \times 10^{-7}$ & 0.049 & $1.3 \times 10^{-6}$ & $2.1 \times 10^{-9}$ \\
\hline MWS-08 & - & - & - & - & - & - & - & - & & - & - & - \\
\hline MWS-09 & - & - & - & - & - & - & - & - & & - & - & - \\
\hline MWS-10 & 0.3 & $8.2 \times 10^{-6}$ & $1.3 \times 10^{-8}$ & - & - & - & 0.028 & $7.7 \times 10^{-7}$ & $1.2 \times 10^{-9}$ & 0.082 & $2.2 \times 10^{-6}$ & $3.6 \times 10^{-9}$ \\
\hline MWS-11 & 0.037 & $1.0 \times 10^{-6}$ & $1.6 \times 10^{-9}$ & - & - & - & 0.046 & $1.3 \times 10^{-6}$ & $2.0 \times 10^{-9}$ & 0.055 & $1.5 \times 10^{-6}$ & $2.4 \times 10^{-9}$ \\
\hline MWS- 12 & 1.9 & $5.2 \times 10^{-5}$ & $8.3 \times 10^{-8}$ & 0.27 & $7.4 \times 10^{-6}$ & $1.2 \times 10^{-8}$ & 0.18 & $4.9 \times 10^{-6}$ & $7.9 \times 10^{-9}$ & 8.8 & $2.4 \times 10^{-4}$ & $3.9 \times 10^{-7}$ \\
\hline MWS-13 & - & - & - & - & - & - & - & - & - & - & - & - \\
\hline MWS-14 & - & - & - & - & - & - & - & - & - & - & - & - \\
\hline MWS-15 & 1.8 & $4.9 \times 10^{-5}$ & $7.9 \times 10^{-8}$ & - & - & - & 5.9 & $1.6 \times 10^{-4}$ & $2.6 \times 10^{-7}$ & 0.081 & $2.2 \times 10^{-6}$ & $3.6 \times 10^{-9}$ \\
\hline MWS-16 & 10 & $2.7 \times 10^{-4}$ & $4.4 \times 10^{-7}$ & - & - & - & 2.9 & $7.9 \times 10^{-5}$ & $1.3 \times 10^{-7}$ & 0.092 & $2.5 \times 10^{-6}$ & $4.0 \times 10^{-9}$ \\
\hline MWS-17 & 0.07 & $1.9 \times 10^{-6}$ & $3.1 \times 10^{-9}$ & - & - & - & 0.15 & $4.1 \times 10^{-6}$ & $6.6 \times 10^{-9}$ & 1.1 & $3.0 \times 10^{-5}$ & $4.8 \times 10^{-8}$ \\
\hline MWS-19 & 0.051 & $1.4 \times 10^{-6}$ & $2.2 \times 10^{-9}$ & - & - & - & - & - & - & 0.08 & $2.2 \times 10^{-6}$ & $3.5 \times 10^{-9}$ \\
\hline MWS-20 & - & - & - & - & - & - & - & - & - & - & - & \\
\hline MWS-21 & - & - & - & - & - & - & - & - & - & 0.94 & $2.6 \times 10^{-5}$ & $4.1 \times 10^{-8}$ \\
\hline MWS-22 & - & - & - & - & - & - & - & - & - & 0.025 & $6.8 \times 10^{-7}$ & $1.1 \times 10^{-9}$ \\
\hline MWS-24 & - & - & - & - & - & - & - & - & - & - & - & - \\
\hline MWS-25 & - & - & - & - & - & - & - & - & - & - & - & - \\
\hline MWS-26 & - & - & - & - & - & - & - & - & - & - & - & - \\
\hline MWS-104 & - & - & - & - & - & - & $\ldots$ & - & - & - & _ & _- \\
\hline MWS-107 & 0.065 & $1.8 \times 10^{-6}$ & $2.8 \times 10^{-9}$ & - & - & - & - & - & - & 0.059 & $1.6 \times 10^{-6}$ & $2.6 \times 10^{-9}$ \\
\hline MWS-110 & 0.19 & $5.2 \times 10^{-6}$ & $8.3 \times 10^{-9}$ & - & - & - & - & - & - & - & - & - \\
\hline MWS-112 & - & - & - & - & - & - & - & - & - & 0.056 & $1.5 \times 10^{-6}$ & $2.5 \times 10^{-9}$ \\
\hline USGS-2 & - & - & - & - & - & - & - & - & - & - & - & - \\
\hline USGS-3 & 0.1 & $2.7 \times 10^{-6}$ & $4.4 \times 10^{-9}$ & - & - & - & - & - & - & 0.022 & $6.0 \times 10^{-7}$ & $9.6 \times 10^{-10}$ \\
\hline USGS-4 & 1.8 & $4.9 \times 10^{-5}$ & $7.9 \times 10^{-8}$ & - & - & - & - & - & - & 1.5 & $4.1 \times 10^{-5}$ & $6.6 \times 10^{-8}$ \\
\hline USGS-5 & - & - & - & - & - & - & - & - & - & - & - & - \\
\hline USGS-7 & - & - & - & - & - & - & - & - & - & - & - & - \\
\hline USGS-8 & - & - & - & - & - & - & - & - & - & - & - & - \\
\hline USGS-9 & - & - & - & - & - & - & - & - & - & 0.092 & $2.5 \times 10^{-6}$ & $4.0 \times 10^{-9}$ \\
\hline
\end{tabular}


TABLE 3.7 (Cont.)

\begin{tabular}{|c|c|c|c|c|c|c|c|c|c|c|c|c|}
\hline \multirow[b]{3}{*}{ Well ID } & \multicolumn{3}{|c|}{ 2,6-DNT } & \multicolumn{3}{|c|}{ 2-Amino-4,6-DNT } & \multicolumn{3}{|c|}{ 4-Amino-2,6-DNT } & \multicolumn{3}{|c|}{ Nitrobenzene } \\
\hline & \multirow[b]{2}{*}{$\begin{array}{c}\mathrm{EPC}^{\mathrm{a}} \\
(\mu \mathrm{g} / \mathrm{L})\end{array}$} & \multicolumn{2}{|c|}{ Intake (Ing/kg-d) } & \multirow[b]{2}{*}{$\begin{array}{c}\mathrm{EPC}^{\mathrm{a}} \\
(\mu \mathrm{g} / \mathrm{L})\end{array}$} & \multicolumn{2}{|c|}{ Intake (mg/kg-d) } & \multirow[b]{2}{*}{$\begin{array}{c}\text { EPC }^{a} \\
(\mu \mathrm{g} / \mathrm{L})\end{array}$} & \multicolumn{2}{|c|}{ Intake (mg/kg-d) } & \multirow[b]{2}{*}{$\begin{array}{c}E^{E P C^{a}} \\
(\mu \mathrm{g} / \mathrm{L})\end{array}$} & \multicolumn{2}{|c|}{ Intake (mg/kg-d) } \\
\hline & & Ingestion & Dermal & & Ingestion & Dermal & & Ingestion & Dermal & & Ingestion & Dermal \\
\hline \multicolumn{13}{|l|}{ Deep Wells } \\
\hline MWD-05 & - & - & - & - & - & - & - & - & - & - & - & - \\
\hline MWD-18 & - & - & - & - & - & - & - & - & - & - & - & - \\
\hline MWGS-0I & - & - & - & - & - & - & - & - & - & - & - & - \\
\hline MWGS-02 & - & - & - & - & - & - & - & - & - & - & - & - \\
\hline MWS-18 & - & - & - & - & - & - & - & - & - & - & - & - \\
\hline MWS-101 & - & - & - & - & - & - & - & - & - & - & - & - \\
\hline MWS-102 & - & - & - & - & - & - & - & - & - & - & - & - \\
\hline MWS-103 & - & - & - & - & - & - & - & - & - & - & - & - \\
\hline TIL-3 & - & - & - & - & - & - & - & - & - & - & - & - \\
\hline \multicolumn{13}{|l|}{ Overburden } \\
\hline MW-2031 & - & - & - & - & - & - & - & - & - & - & - & - \\
\hline MW-2032 & 4.4 & $1.2 \times 10^{-4}$ & $1.9 \times 10^{-7}$ & 3.6 & $9.9 \times 10^{-5}$ & $1.6 \times 10^{-7}$ & 2.8 & $7.7 \times 10^{-5}$ & $1.2 \times 10^{-7}$ & - & - & - \\
\hline MW-2033 & 4.9 & $1.3 \times 10^{-4}$ & $2.1 \times 10^{-7}$ & 3.6 & $9.9 \times 10^{-5}$ & $1.6 \times 10^{-7}$ & 3.3 & $9.0 \times 10^{-5}$ & $1.4 \times 10^{-7}$ & - & - & - \\
\hline MW-3001 & - & - & - & - & - & - & - & - & - & - & - & - \\
\hline MW-3013 & - & - & - & - & - & - & - & - & - & - & - & - \\
\hline MW-3018 & - & - & - & - & - & - & - & - & - & - & - & - \\
\hline MW-3022 & - & - & - & - & - & - & - & - & - & - & - & - \\
\hline USGS- $2 \mathrm{~A}$ & - & - & - & - & - & - & - & - & - & - & - & - \\
\hline MWV-01 & 1.0 & $2.7 \times 10^{-5}$ & $4.4 \times 10^{-8}$ & 3.8 & $1.0 \times 10^{-4}$ & $1.7 \times 10^{-7}$ & 6.8 & $1.9 \times 10^{-4}$ & $3.0 \times 10^{-7}$ & - & - & - \\
\hline MWV-02 & 0.048 & $1.3 \times 10^{-6}$ & $2.1 \times 10^{-9}$ & 0.50 & $1.4 \times 10^{-5}$ & $2.2 \times 10^{-8}$ & 1 & $2.7 \times 10^{-5}$ & $4.4 \times 10^{-8}$ & - & - & - \\
\hline MWV-09 & 2.9 & $7.9 \times 10^{-5}$ & $1.3 \times 10^{-7}$ & 35 & $9.6 \times 10^{-4}$ & $1.5 \times 10^{-6}$ & 26 & $7.1 \times 10^{-4}$ & $1.1 \times 10^{-6}$ & - & - & - \\
\hline MWV-13 & - & - & - & - & - & - & - & - & - & - & - & - \\
\hline MWV-16 & 0.069 & $1.9 \times 10^{-6}$ & $3.0 \times 10^{-9}$ & 0.32 & $8.8 \times 10^{-6}$ & $1.4 \times 10^{-8}$ & 0.57 & $1.6 \times 10^{-5}$ & $2.5 \times 10^{-8}$ & - & - & - \\
\hline MWV-17 & - & - & - & - & - & - & - & - & - & - & - & - \\
\hline MWV-18 & - & - & - & - & - & - & - & - & - & - & - & - \\
\hline MWV-22 & 0.14 & $3.8 \times 10^{-6}$ & $6.1 \times 10^{-9}$ & 0.057 & $1.6 \times 10^{-6}$ & $2.5 \times 10^{-9}$ & 0.21 & $5.8 \times 10^{-6}$ & $9.2 \times 10^{-9}$ & - & - & - \\
\hline MWV-24R & 1.4 & $3.8 \times 10^{-5}$ & $6.1 \times 10^{-8}$ & 0.47 & $1.3 \times 10^{-5}$ & $2.1 \times 10^{-8}$ & 1 & $2.7 \times 10^{-5}$ & $4.4 \times 10^{-8}$ & - & - & - \\
\hline
\end{tabular}


TABLE 3.7 (Cont.)

\begin{tabular}{|c|c|c|c|c|c|c|c|c|c|c|c|c|}
\hline \multirow[b]{3}{*}{ Well ID } & \multirow[b]{3}{*}{$\begin{array}{c}\mathrm{EPC}^{\mathrm{a}} \\
(\mu \mathrm{g} / \mathrm{L})\end{array}$} & \multicolumn{2}{|c|}{ 2,6-DNT } & \multicolumn{3}{|c|}{ 2-Amino-4,6-DNT } & \multicolumn{3}{|c|}{ 4-Amino-2,6-DNT } & \multicolumn{3}{|c|}{ Nitrobenzene } \\
\hline & & \multicolumn{2}{|c|}{ Intake (mg/kg-d) } & \multirow[b]{2}{*}{$\begin{array}{l}\mathrm{EPC}^{\mathrm{a}} \\
(\mu \mathrm{g} / \mathrm{L})\end{array}$} & \multicolumn{2}{|c|}{ Intake (mg/kg-d) } & \multirow[b]{2}{*}{$\begin{array}{c}\mathrm{EPC}^{\mathrm{a}} \\
(\mu \mathrm{g} / \mathrm{L})\end{array}$} & \multicolumn{2}{|c|}{ Intake (mg/kg-d) } & \multirow[b]{2}{*}{$\begin{array}{c}\mathrm{EPC}^{\mathrm{a}} \\
(\mu \mathrm{g} / \mathrm{L})\end{array}$} & \multicolumn{2}{|c|}{ Intake (mg/kg-d) } \\
\hline & & Ingestion & Dermal & & Ingestion & Dermal & & Ingestion & Dermal & & Ingestion & Dernal \\
\hline \multicolumn{13}{|l|}{ Unweathered } \\
\hline MW-2019 & - & - & - & - & - & - & - & - & - & - & - & - \\
\hline MW-2021 & - & - & - & - & - & - & - & - & - & - & - & - \\
\hline MW-2022 & - & - & - & - & - & - & - & - & - & - & - & - \\
\hline$M W-2023$ & - & - & - & - & - & - & - & - & - & - & - & - \\
\hline$M W-2024$ & - & - & - & - & - & - & - & - & - & - & - & - \\
\hline$M W-2025$ & - & - & - & - & - & - & - & - & - & - & - & - \\
\hline MW-2026 & - & - & - & - & - & - & - & - & - & - & - & - \\
\hline$M W-2027$ & - & - & - & - & - & - & - & - & - & - & - & - \\
\hline MW-2028 & - & - & - & - & - & - & - & - & - & - & - & - \\
\hline MW-2029 & - & - & - & - & - & - & - & - & - & - & - & - \\
\hline$M W-3002$ & - & - & - & - & - & - & - & - & - & - & - & - \\
\hline MW-3006 & - & - & - & - & - & - & - & - & - & - & - & - \\
\hline MW-3024 & 0.45 & $1.2 \times 10^{-5}$ & $2.0 \times 10^{-8}$ & 0.032 & $8.8 \times 10^{-7}$ & $1.4 \times 10^{-9}$ & 0.057 & $1.6 \times 10^{-6}$ & $2.5 \times 10^{-9}$ & - & - & - \\
\hline MW-3026 & 0.046 & $1.3 \times 10^{-6}$ & $2.0 \times 10^{-9}$ & 0.30 & $8.2 \times 10^{-6}$ & $1.3 \times 10^{-8}$ & 0.37 & $1.0 \times 10^{-5}$ & $1.6 \times 10^{-8}$ & - & - & - \\
\hline$M W-4004$ & - & - & - & - & - & - & - & - & - & - & - & - \\
\hline$M W-4007$ & - & - & - & - & - & - & 0.017 & $4.7 \times 10^{-7}$ & $7.5 \times 10^{-10}$ & - & - & - \\
\hline MW-4008 & - & - & - & - & - & - & - & - & - & - & - & - \\
\hline MW-4009 & - & - & - & - & - & - & - & - & - & - & - & - \\
\hline MW-4011 & 0.065 & $1.8 \times 10^{-6}$ & $2.8 \times 10^{-9}$ & 0.77 & $2.1 \times 10^{-5}$ & $3.4 \times 10^{-8}$ & 1.8 & $4.9 \times 10^{-5}$ & $7.9 \times 10^{-8}$ & - & - & - \\
\hline$M W-4012$ & - & - & - & - & - & - & - & - & - & - & - & - \\
\hline MW-4022 & - & - & - & - & - & - & - & - & - & - & - & - \\
\hline MWD-02 & - & - & - & - & - & - & - & - & - & - & - & - \\
\hline MWD-06 & - & - & _ & - & - & - & - & - & - & - & - & - \\
\hline MWD-09 & 0.16 & $4.4 \times 10^{-6}$ & $7.0 \times 10^{-9}$ & 0.095 & $2.6 \times 10^{-6}$ & $4.2 \times 10^{-9}$ & 0.5 & $1.4 \times 10^{-5}$ & $2.2 \times 10^{-8}$ & - & - & - \\
\hline MWD-23 & - & - & - & - & - & - & - & - & - & - & - & - \\
\hline MWD-106 & - & - & - & - & - & - & - & - & - & - & - & - \\
\hline MWS-05 & - & - & - & - & - & - & - & - & - & - & - & - \\
\hline MWS-06 & - & - & - & - & - & - & - & - & - & - & - & - \\
\hline MWS-105 & - & - & - & - & - & - & - & - & - & - & - & - \\
\hline MWS-106 & - & - & - & - & - & - & - & - & - & - & - & - \\
\hline MWS-109 & - & - & - & - & - & - & - & - & - & - & - & - \\
\hline TIL-4 & - & - & - & - & - & - & - & - & - & - & - & - \\
\hline USGS-1 & 0.022 & $6.0 \times 10^{-7}$ & $9.6 \times 10^{-10}$ & 0.057 & $1.6 \times 10^{-6}$ & $2.5 \times 10^{-9}$ & 0.2 & $5.5 \times 10^{-6}$ & $8.8 \times 10^{-9}$ & - & - & - \\
\hline USGS-6 & - & - & - & - & - & - & - & - & - & - & - & - \\
\hline
\end{tabular}


TABLE 3.7 (Cont.)

\begin{tabular}{|c|c|c|c|c|c|c|c|c|c|c|c|c|}
\hline \multirow[b]{3}{*}{ Well ID } & \multirow[b]{3}{*}{$\begin{array}{c}\mathrm{EPC}^{\mathrm{a}} \\
(\mu \mathrm{g} / \mathrm{L})\end{array}$} & \multicolumn{2}{|l|}{ 2,6-DNT } & \multicolumn{3}{|c|}{ 2-Amino-4,6-DNT } & \multicolumn{3}{|c|}{ 4-Amino-2,6-DNT } & \multicolumn{3}{|c|}{ Nitrobenzene } \\
\hline & & \multicolumn{2}{|c|}{ Intake (mg/kg-d) } & \multirow[b]{2}{*}{$\begin{array}{c}\mathrm{EPC}^{\mathrm{i}} \\
(\mu \mathrm{g} / \mathrm{L})\end{array}$} & \multicolumn{2}{|c|}{ Intake (mg/kg-d) } & \multirow[b]{2}{*}{$\begin{array}{l}\mathrm{EPC}^{\mathrm{a}} \\
(\mu \mathrm{g} / \mathrm{L})\end{array}$} & \multicolumn{2}{|c|}{ Intake (mg/kg-d) } & \multirow[b]{2}{*}{$\begin{array}{c}\mathrm{EPC}^{\mathrm{i}} \\
(\mu \mathrm{g} / \mathrm{L})\end{array}$} & \multicolumn{2}{|c|}{ Intake (mg/kg-d) } \\
\hline & & Ingestion & Dermal & & Ingestion & Dermal & & Ingestion & Dermal & & Ingestion & Dermal \\
\hline \multicolumn{13}{|l|}{ Weathered } \\
\hline MW-200I & 0.056 & $1.5 \times 10^{-6}$ & $2.5 \times 10^{-9}$ & - & - & - & - & - & - & - & - & - \\
\hline MW-2002 & 0.41 & $1.1 \times 10^{-5}$ & $1.8 \times 10^{-8}$ & 0.83 & $2.3 \times 10^{-5}$ & $3.6 \times 10^{-8}$ & 0.93 & $2.5 \times 10^{-5}$ & $4.1 \times 10^{-8}$ & - & - & - \\
\hline MW-2003 & 0.45 & $1.2 \times 10^{-5}$ & $2.0 \times 10^{-8}$ & 0.18 & $4.9 \times 10^{-6}$ & $7.9 \times 10^{-9}$ & 0.46 & $1.3 \times 10^{-5}$ & $2.0 \times 10^{-8}$ & - & - & - \\
\hline MW-2004 & - & - & - & - & - & - & - & - & - & - & - & - \\
\hline$M W-2005$ & 0.090 & $2.5 \times 10^{-6}$ & $3.9 \times 10^{-9}$ & 0.12 & $3.3 \times 10^{-6}$ & $5.3 \times 10^{-9}$ & 0.12 & $3.3 \times 10^{-6}$ & $5.3 \times 10^{-9}$ & - & - & - \\
\hline MW-2006 & 0.0090 & $2.5 \times 10^{-7}$ & $3.9 \times 10^{-10}$ & - & - & - & - & - & - & 0.042 & $1.2 \times 10^{-6}$ & $1.8 \times 10^{-9}$ \\
\hline MW-2007 & - & - & - & - & - & - & - & - & - & - & - & - \\
\hline MW-2008 & - & - & - & - & - & - & - & - & - & - & - & - \\
\hline MW-2009 & - & - & - & - & - & - & - & - & - & - & - & - \\
\hline$M W-2010$ & 0.75 & $2.1 \times 10^{-5}$ & $3.3 \times 10^{-8}$ & 0.72 & $2.0 \times 10^{-5}$ & $3.2 \times 10^{-8}$ & 0.81 & $2.2 \times 10^{-5}$ & $3.6 \times 10^{-8}$ & - & - & - \\
\hline$M W-2011$ & 1.6 & $4.4 \times 10^{-5}$ & $7.0 \times 10^{-8}$ & 2.0 & $5.5 \times 10^{-5}$ & $8.8 \times 10^{-8}$ & 0.98 & $2.7 \times 10^{-5}$ & $4.3 \times 10^{-8}$ & - & - & - \\
\hline $\mathrm{MW}-2012$ & 0.65 & $1.8 \times 10^{-5}$ & $2.8 \times 10^{-8}$ & 0.31 & $8.5 \times 10^{-6}$ & $1.4 \times 10^{-8}$ & 0.37 & $1.0 \times 10^{-5}$ & $1.6 \times 10^{-8}$ & - & - & - \\
\hline MW-2013 & 4.4 & $1.2 \times 10^{-4}$ & $1.9 \times 10^{-7}$ & 2.4 & $6.6 \times 10^{-5}$ & $1.1 \times 10^{-7}$ & 2.2 & $6.0 \times 10^{-5}$ & $9.6 \times 10^{-8}$ & - & - & - \\
\hline MW-2014 & 0.41 & $1.1 \times 10^{-5}$ & $1.8 \times 10^{-8}$ & 0.41 & $1.1 \times 10^{-5}$ & $1.8 \times 10^{-8}$ & 0.63 & $1.7 \times 10^{-5}$ & $2.8 \times 10^{-8}$ & - & - & - \\
\hline MW-2015 & - & - & - & - & - & - & - & - & - & - & - & - \\
\hline$M W-2016$ & - & - & - & - & - & - & - & - & - & - & - & - \\
\hline MW-2017 & - & - & - & - & - & - & - & - & - & - & - & - \\
\hline MW-2018 & - & - & - & - & - & - & - & - & - & - & - & - \\
\hline$M W-2020$ & - & - & - & - & - & - & - & - & - & - & - & - \\
\hline MW-2030 & 11 & $3.0 \times 10^{-4}$ & $4.8 \times 10^{-7}$ & 5.5 & $1.5 \times 10^{-4}$ & $2.4 \times 10^{-7}$ & 4.4 & $1.2 \times 10^{-4}$ & $1.9 \times 10^{-7}$ & - & - & - \\
\hline$M W-2034$ & - & - & - & - & - & - & - & - & - & - & - & - \\
\hline$M W-2035$ & - & - & - & - & - & - & - & - & - & - & - & - \\
\hline MW-2036 & - & - & - & _ & - & _- & _- & _- & _- & _- & _- & _- \\
\hline MW-2037 & 0.13 & $3.6 \times 10^{-6}$ & $5.7 \times 10^{-9}$ & 0.11 & $3.0 \times 10^{-6}$ & $4.8 \times 10^{-9}$ & 0.11 & $3.0 \times 10^{-6}$ & $4.8 \times 10^{-9}$ & - & - & - \\
\hline MW-2038 & 0.32 & $8.8 \times 10^{-6}$ & $1.4 \times 10^{-8}$ & 0.40 & $1.1 \times 10^{-5}$ & $1.8 \times 10^{-8}$ & 0.46 & $1.3 \times 10^{-5}$ & $2.0 \times 10^{-8}$ & 0.062 & $1.7 \times 10^{-6}$ & $2.7 \times 10^{-9}$ \\
\hline MW-2039 & 1.7 & $4.7 \times 10^{-5}$ & $7.5 \times 10^{-8}$ & 2.0 & $5.5 \times 10^{-5}$ & $8.8 \times 10^{-8}$ & 1.6 & $4.4 \times 10^{-5}$ & $7.0 \times 10^{-8}$ & 0.054 & $1.5 \times 10^{-6}$ & $2.4 \times 10^{-9}$ \\
\hline MW-2040 & - & - & - & - & - & - & - & - & - & - & - & - \\
\hline MW-204I & - & - & - & - & - & - & - & - & - & - & - & - \\
\hline MW-2042 & - & - & - & - & - & - & - & - & - & - & - & - \\
\hline MW-2043 & - & - & - & - & - & - & - & - & - & - & - & - \\
\hline MW-2044 & - & - & - & 0.022 & $6.0 \times 10^{-7}$ & $9.6 \times 10^{-10}$ & 0.033 & $9.0 \times 10^{-7}$ & $1.4 \times 10^{-9}$ & - & - & - \\
\hline MW-3003 & 0.085 & $2.3 \times 10^{-6}$ & $3.7 \times 10^{-9}$ & - & - & - & 0.034 & $9.3 \times 10^{-7}$ & $1.5 \times 10^{-9}$ & - & - & - \\
\hline MW-3007 & - & - & - & - & - & - & - & - & - & - & - & - \\
\hline
\end{tabular}


TABLE 3.7 (Cont.)

\begin{tabular}{|c|c|c|c|c|c|c|c|c|c|c|c|c|}
\hline \multirow[b]{3}{*}{ Well ID } & \multicolumn{3}{|c|}{ 2,6-DNT } & \multicolumn{3}{|c|}{ 2-Amino-4,6-DNT } & \multicolumn{3}{|c|}{ 4-Amino-2,6-DNT } & \multicolumn{3}{|c|}{ Nitrobenzene } \\
\hline & \multirow[b]{2}{*}{$\begin{array}{c}\mathrm{EPC}^{\mathrm{a}} \\
(\mu \mathrm{g} / \mathrm{L})\end{array}$} & \multicolumn{2}{|c|}{ Intake (mg/kg-d) } & \multirow[b]{2}{*}{$\begin{array}{c}\mathrm{EPC}^{\mathrm{a}} \\
(\mu \mathrm{g} / \mathrm{L})\end{array}$} & \multicolumn{2}{|c|}{ Intake (mg/kg-d) } & \multirow[b]{2}{*}{$\begin{array}{l}\mathrm{EPC}^{\mathrm{a}} \\
(\mu \mathrm{g} / \mathrm{L})\end{array}$} & \multicolumn{2}{|c|}{ Intake (mg/kg-d) } & \multirow[b]{2}{*}{$\begin{array}{c}\mathrm{EPC}^{\mathrm{a}} \\
(\mu \mathrm{g} / \mathrm{L})\end{array}$} & \multicolumn{2}{|c|}{ Intake (mg/kg-d) } \\
\hline & & Ingestion & Dermal & & Ingestion & Dermal & & Ingestion & Dermal & & Ingestion & Dernnal \\
\hline \multicolumn{13}{|c|}{ Weathered (cont.) } \\
\hline MW-3008 & - & - & - & - & - & - & - & - & - & - & - & - \\
\hline MW-3009 & - & - & - & - & - & - & - & - & - & - & - & - \\
\hline MW-3010 & - & - & - & - & - & - & - & - & - & - & - & - \\
\hline$M W-3019$ & - & - & - & - & - & - & - & - & - & - & - & - \\
\hline$M W-3023$ & 5 & $1.4 \times 10^{-4}$ & $2.2 \times 10^{-7}$ & 0.17 & $4.7 \times 10^{-6}$ & $7.5 \times 10^{-9}$ & 0.32 & $8.8 \times 10^{-6}$ & $1.4 \times 10^{-8}$ & - & - & - \\
\hline MW-3025 & - & - & - & - & - & - & 0.03 & $8.2 \times 10^{-7}$ & $1.3 \times 10^{-9}$ & - & - & - \\
\hline MW-3027 & 0.04 & $1.1 \times 10^{-6}$ & $1.8 \times 10^{-9}$ & 0.19 & $5.2 \times 10^{-6}$ & $8.3 \times 10^{-9}$ & 0.18 & $4.9 \times 10^{-6}$ & $7.9 \times 10^{-9}$ & - & - & - \\
\hline$M W-4001$ & 3.1 & $8.5 \times 10^{-5}$ & $1.4 \times 10^{-7}$ & 16 & $4.4 \times 10^{-4}$ & $7.0 \times 10^{-7}$ & 22 & $6.0 \times 10^{-4}$ & $9.6 \times 10^{-7}$ & - & - & - \\
\hline MW-4002 & 0.29 & $7.9 \times 10^{-6}$ & $1.3 \times 10^{-8}$ & 1.4 & $3.8 \times 10^{-5}$ & $6.1 \times 10^{-8}$ & 2.3 & $6.3 \times 10^{-5}$ & $1.0 \times 10^{-7}$ & - & - & - \\
\hline MW-4003 & - & - & - & 0.016 & $4.4 \times 10^{-7}$ & $7.0 \times 10^{-10}$ & 0.028 & $7.7 \times 10^{-7}$ & $1.2 \times 10^{-9}$ & - & - & - \\
\hline MW-4005 & - & - & - & - & - & - & - & - & - & - & - & - \\
\hline MW-4006 & 3.1 & $8.5 \times 10^{-5}$ & $1.4 \times 10^{-7}$ & 1.8 & $4.9 \times 10^{-5}$ & $7.9 \times 10^{-8}$ & 2.5 & $6.8 \times 10^{-5}$ & $1.1 \times 10^{-7}$ & - & - & - \\
\hline$M W-4010$ & - & - & - & - & - & - & - & - & - & - & - & - \\
\hline $\mathrm{MW}-4013$ & 0.74 & $2.0 \times 10^{-5}$ & $3.2 \times 10^{-8}$ & 1.7 & $4.7 \times 10^{-5}$ & $7.5 \times 10^{-8}$ & 2.0 & $5.5 \times 10^{-5}$ & $8.8 \times 10^{-8}$ & - & - & - \\
\hline$M W-4014$ & 0.087 & $2.4 \times 10^{-6}$ & $3.8 \times 10^{-9}$ & 0.28 & $7.7 \times 10^{-6}$ & $1.2 \times 10^{-8}$ & 0.52 & $1.4 \times 10^{-5}$ & $2.3 \times 10^{-8}$ & - & - & - \\
\hline$M W-4015$ & 1.1 & $3.0 \times 10^{-5}$ & $4.8 \times 10^{-8}$ & 3.2 & $8.8 \times 10^{-5}$ & $1.4 \times 10^{-7}$ & 4.1 & $1.1 \times 10^{-4}$ & $1.8 \times 10^{-7}$ & - & - & - \\
\hline MW-4016 & - & - & - & - & - & - & - & - & - & - & - & - \\
\hline$M W-4017$ & - & - & - & - & - & - & - & - & - & - & - & - \\
\hline MW-4018 & - & - & - & - & - & - & - & - & - & - & - & - \\
\hline MW-4019 & - & - & - & - & - & - & - & - & - & - & - & - \\
\hline$M W-4020$ & - & - & - & - & - & - & - & - & - & - & - & - \\
\hline$M W-4021$ & - & - & - & - & - & - & - & - & - & - & - & - \\
\hline $\mathrm{MW}-4023$ & 0.023 & $6.3 \times 10^{-7}$ & $1.0 \times 10^{-9}$ & 0.038 & $1.0 \times 10^{-6}$ & $1.7 \times 10^{-9}$ & 0.05 & $1.4 \times 10^{-6}$ & $2.2 \times 10^{-9}$ & - & - & - \\
\hline MW-4024 & - & - & - & - & - & - & - & - & - & - & - & - \\
\hline MW-4025 & - & - & - & - & - & - & - & - & - & - & - & - \\
\hline MWD-15 & 0.64 & $1.8 \times 10^{-5}$ & $2.8 \times 10^{-8}$ & 0.71 & $1.9 \times 10^{-5}$ & $3.1 \times 10^{-8}$ & 1.7 & $4.7 \times 10^{-5}$ & $7.5 \times 10^{-8}$ & - & - & - \\
\hline MWD-25 & - & - & - & - & - & - & - & - & - & - & - & - \\
\hline MWD-107 & 0.09 & $2.5 \times 10^{-6}$ & $3.9 \times 10^{-9}$ & 0.04 & $1.1 \times 10^{-6}$ & $1.8 \times 10^{-9}$ & 0.51 & $1.4 \times 10^{-5}$ & $2.2 \times 10^{-8}$ & - & - & - \\
\hline MWD-112 & - & - & - & - & - & - & - & - & - & - & - & - \\
\hline MWS-01 & 0.64 & $1.8 \times 10^{-5}$ & $2.8 \times 10^{-8}$ & - & - & - & 0.044 & $1.2 \times 10^{-6}$ & $1.9 \times 10^{-9}$ & - & - & - \\
\hline MWS-02 & - & - & - & - & - & - & - & - & - & - & - & - \\
\hline MWS-03 & - & - & - & - & - & - & - & - & - & - & - & - \\
\hline MWS-04 & 1.2 & $3.3 \times 10^{-5}$ & $5.3 \times 10^{-8}$ & 7.6 & $2.1 \times 10^{-4}$ & $3.3 \times 10^{-7}$ & 8.3 & $2.3 \times 10^{-4}$ & $3.6 \times 10^{-7}$ & - & - & - \\
\hline MWS-07 & 1.2 & $3.3 \times 10^{-5}$ & $5.3 \times 10^{-8}$ & 5.6 & $1.5 \times 10^{-4}$ & $2.5 \times 10^{-7}$ & 11 & $3.0 \times 10^{-4}$ & $4.8 \times 10^{-7}$ & - & - & - \\
\hline
\end{tabular}


TABLE 3.7 (Cont.)

\begin{tabular}{|c|c|c|c|c|c|c|c|c|c|c|c|c|}
\hline \multirow[b]{3}{*}{ Well ID } & \multicolumn{3}{|c|}{ 2,6-DNT } & \multicolumn{3}{|c|}{ 2-Amino-4,6-DNT } & \multicolumn{3}{|c|}{ 4-Amino-2,6-DNT } & \multicolumn{3}{|c|}{ Nitrabenzene } \\
\hline & \multirow[b]{2}{*}{$\begin{array}{c}\mathrm{EPC}^{\mathrm{a}} \\
(\mu \mathrm{g} / \mathrm{L})\end{array}$} & \multicolumn{2}{|c|}{ Intake $(\mathrm{mg} / \mathrm{kg}-\mathrm{d})$} & \multirow[b]{2}{*}{$\begin{array}{c}\mathrm{EPC}^{\mathrm{a}} \\
(\mu \mathrm{g} / \mathrm{L})\end{array}$} & \multicolumn{2}{|c|}{ Intake (mg/kg-d) } & \multirow[b]{2}{*}{$\begin{array}{c}\mathrm{EPC}^{\mathrm{a}} \\
(\mu \mathrm{g} / \mathrm{L})\end{array}$} & \multicolumn{2}{|c|}{ Intake (mg/kg-d) } & \multirow[b]{2}{*}{$\begin{array}{c}\mathrm{EPC}^{\mathrm{a}} \\
(\mu \mathrm{g} / \mathrm{L})\end{array}$} & \multicolumn{2}{|c|}{ Intake $(\mathrm{mg} / \mathrm{kg}-\mathrm{d})$} \\
\hline & & Ingestion & Dermal & & Ingestion & Dermal & & Ingestion & Dermal & & Ingestion & Dermal \\
\hline \multicolumn{13}{|c|}{ Weathered (cont.) } \\
\hline MWS-08 & - & - & - & - & - & - & - & - & - & - & - & - \\
\hline MWS-09 & - & - & - & - & - & - & - & - & - & - & - & - \\
\hline MWS- 10 & 2 & $5.5 \times 10^{-5}$ & $8.8 \times 10^{-8}$ & 6.3 & $1.7 \times 10^{-4}$ & $2.8 \times 10^{-7}$ & 16 & $4.4 \times 10^{-4}$ & $7.0 \times 10^{-7}$ & - & - & - \\
\hline MWS- 11 & 0.54 & $1.5 \times 10^{-5}$ & $2.4 \times 10^{-8}$ & 0.48 & $1.3 \times 10^{-5}$ & $2.1 \times 10^{-8}$ & 2.3 & $6.3 \times 10^{-5}$ & $1.0 \times 10^{-7}$ & - & - & - \\
\hline MWS-12 & 15 & $4.1 \times 10^{-4}$ & $6.6 \times 10^{-7}$ & 1.1 & $3.0 \times 10^{-5}$ & $4.8 \times 10^{-8}$ & 2.5 & $6.8 \times 10^{-5}$ & $1.1 \times 10^{-7}$ & - & - & - \\
\hline MWS-13 & - & - & - & - & - & - & - & - & - & - & - & - \\
\hline MWS-14 & - & - & - & - & - & - & - & - & - & - & - & - \\
\hline MWS-15 & 1 & $2.7 \times 10^{-5}$ & $4.4 \times 10^{-8}$ & 11 & $3.0 \times 10^{-4}$ & $4.8 \times 10^{-7}$ & 20 & $5.5 \times 10^{-4}$ & $8.8 \times 10^{-7}$ & - & - & - \\
\hline MWS-16 & 1.2 & $3.3 \times 10^{-5}$ & $5.3 \times 10^{-8}$ & 4.7 & $1.3 \times 10^{-4}$ & $2.1 \times 10^{-7}$ & 8.4 & $2.3 \times 10^{-4}$ & $3.7 \times 10^{-7}$ & - & - & - \\
\hline MWS-17 & 13 & $3.6 \times 10^{-4}$ & $5.7 \times 10^{-7}$ & 3.9 & $1.1 \times 10^{-4}$ & $1.7 \times 10^{-7}$ & 4.6 & $1.3 \times 10^{-4}$ & $2.0 \times 10^{-7}$ & - & - & - \\
\hline MWS-19 & 0.14 & $3.8 \times 10^{-6}$ & $6.1 \times 10^{-9}$ & 0.27 & $7.4 \times 10^{-6}$ & $1.2 \times 10^{-8}$ & 0.39 & $1.1 \times 10^{-5}$ & $1.7 \times 10^{-8}$ & - & - & - \\
\hline MWS-20 & 0.013 & $3.6 \times 10^{-7}$ & $5.7 \times 10^{-10}$ & - & - & - & 0.057 & $1.6 \times 10^{-6}$ & $2.5 \times 10^{-9}$ & - & - & - \\
\hline MWS-2l & 0.17 & $4.7 \times 10^{-6}$ & $7.5 \times 10^{-9}$ & 0.23 & $6.3 \times 10^{-6}$ & $1.0 \times 10^{-8}$ & 0.51 & $1.4 \times 10^{-5}$ & $2.2 \times 10^{-8}$ & - & - & - \\
\hline MWS-22 & 0.13 & $3.6 \times 10^{-6}$ & $5.7 \times 10^{-9}$ & 0.073 & $2.0 \times 10^{-6}$ & $3.2 \times 10^{-9}$ & 0.19 & $5.2 \times 10^{-6}$ & $8.3 \times 10^{-9}$ & - & - & - \\
\hline MWS-24 & - & - & - & - & - & - & - & - & - & - & - & - \\
\hline MWS-25 & - & - & - & - & - & - & - & - & - & - & - & - \\
\hline MWS-26 & - & - & - & - & - & - & - & - & - & - & - & - \\
\hline MWS-104 & - & - & - & - & - & - & - & - & - & - & - & - \\
\hline MWS- 107 & 0.16 & $4.4 \times 10^{-6}$ & $7.0 \times 10^{-9}$ & 0.059 & $1.6 \times 10^{-6}$ & $2.6 \times 10^{-9}$ & 0.65 & $1.8 \times 10^{-5}$ & $2.8 \times 10^{-8}$ & - & - & - \\
\hline MWS- 110 & 0.054 & $1.5 \times 10^{-6}$ & $2.4 \times 10^{-9}$ & 0.18 & $4.9 \times 10^{-6}$ & $7.9 \times 10^{-9}$ & 0.37 & $1.0 \times 10^{-5}$ & $1.6 \times 10^{-8}$ & - & - & - \\
\hline MWS-112 & 0.018 & $4.9 \times 10^{-7}$ & $7.9 \times 10^{-10}$ & 0.056 & $1.5 \times 10^{-6}$ & $2.5 \times 10^{-9}$ & 0.14 & $3.8 \times 10^{-6}$ & $6.1 \times 10^{-9}$ & 0.062 & $1.7 \times 10^{-6}$ & $2.7 \times 10^{-9}$ \\
\hline USGS-2 & - & - & - & - & - & - & - & - & - & - & - & - \\
\hline USGS-3 & 0.19 & $5.2 \times 10^{-6}$ & $8.3 \times 10^{-9}$ & 0.025 & $6.8 \times 10^{-7}$ & $1.1 \times 10^{-9}$ & 0.25 & $6.8 \times 10^{-6}$ & $1.1 \times 10^{-8}$ & - & - & - \\
\hline USGS-4 & 2.1 & $5.8 \times 10^{-5}$ & $9.2 \times 10^{-8}$ & 2 & $5.5 \times 10^{-5}$ & $8.8 \times 10^{-8}$ & 2.2 & $6.0 \times 10^{-5}$ & $9.6 \times 10^{-8}$ & - & - & - \\
\hline USGS-5 & - & - & - & - & - & - & - & - & - & - & - & - \\
\hline USGS-7 & - & - & - & - & - & - & - & - & - & - & - & - \\
\hline USGS-8 & - & - & - & - & - & - & - & - & - & _- & - & - \\
\hline USGS-9 & 0.015 & $4.1 \times 10^{-7}$ & $6.6 \times 10^{-10}$ & 2.6 & $7.1 \times 10^{-5}$ & $1.1 \times 10^{-7}$ & 3.7 & $1.0 \times 10^{-4}$ & $1.6 \times 10^{-7}$ & - & - & - \\
\hline
\end{tabular}


TABLE 3.7 (Cont.)

\begin{tabular}{|c|c|c|c|c|c|c|c|c|c|c|c|c|c|}
\hline \multirow[b]{3}{*}{ Well ID } & \multicolumn{3}{|c|}{ o-Nitrotoluene } & \multicolumn{4}{|c|}{ m-Nitrotoluene } & \multicolumn{3}{|c|}{ p-Nitrotoluene } & \multicolumn{3}{|c|}{ 1,2-DCE } \\
\hline & \multirow[b]{2}{*}{$\begin{array}{c}\mathrm{EPC}^{\mathrm{a}} \\
(\mu \mathrm{g} / \mathrm{L}) \\
\end{array}$} & \multicolumn{2}{|c|}{ Intake (mg/kg-d) } & & \multirow[b]{2}{*}{$\begin{array}{c}E P C^{\mathrm{a}} \\
(\mu \mathrm{g} / \mathrm{L}) \\
\end{array}$} & \multicolumn{2}{|c|}{ Intake (mg/kg-d) } & \multirow[b]{2}{*}{$\begin{array}{c}\mathrm{EPC}^{\mathrm{a}} \\
(\mu \mathrm{g} / \mathrm{L})\end{array}$} & \multicolumn{2}{|c|}{ Intake $(\mathrm{mg} / \mathrm{kg}-\mathrm{d})$} & \multirow[b]{2}{*}{$\begin{array}{r}\mathrm{EPC}^{\mathrm{a}} \\
(\mu \mathrm{g} / \mathrm{L}) \\
\end{array}$} & \multicolumn{2}{|c|}{ Intake (mg/kg-d) } \\
\hline & & Ingestion & Dermal & & & Ingestion & Dermal & & Ingestion & Dermal & & Ingestion & Dermal \\
\hline \multicolumn{14}{|l|}{ Deep Wells } \\
\hline MWD-05 & - & - & - & & - & - & - & - & - & - & - & - & - \\
\hline MWD-18 & - & - & - & & - & - & - & - & - & - & - & - & - \\
\hline MWGS-01 & - & - & - & & - & - & - & - & - & - & - & - & - \\
\hline MWGS-02 & - & - & - & & - & - & - & - & - & - & - & - & - \\
\hline MWS-18 & - & - & - & & - & - & - & - & - & - & - & - & - \\
\hline MWS-101 & - & - & - & & - & - & - & - & - & - & - & - & - \\
\hline MWS-102 & - & - & - & & - & - & - & - & - & - & - & - & - \\
\hline MWS-103 & - & - & - & & - & - & - & - & - & - & - & - & - \\
\hline TIL-3 & - & - & - & & - & - & - & - & - & - & - & - & - \\
\hline \multicolumn{14}{|l|}{ Overburden } \\
\hline MW-203I & - & - & - & & - & - & - & - & - & - & - & - & - \\
\hline MW-2032 & 0.21 & $5.8 \times 10^{-6}$ & $9.2 \times 10^{-9}$ & & 0.043 & $1.2 \times 10^{-6}$ & $1.9 \times 10^{-9}$ & - & - & - & $3.9^{\mathrm{c}}$ & $1.1 \times 10^{-4}$ & $7.3 \times 10^{-8}$ \\
\hline MW-2033 & 0.65 & $1.8 \times 10^{-5}$ & $2.8 \times 10^{-8}$ & & 0.047 & $1.3 \times 10^{-6}$ & $2.1 \times 10^{-9}$ & 0.09 & $2.5 \times 10^{-6}$ & $3.9 \times 10^{-9}$ & - & - & - \\
\hline MW-300I & - & - & - & & - & - & - & - & - & - & - & - & - \\
\hline$M W-3013$ & - & - & - & & - & - & - & - & - & - & - & - & - \\
\hline$M W-3018$ & - & - & - & & - & - & - & - & - & - & - & - & - \\
\hline$M W-3022$ & - & - & - & & - & - & - & - & - & - & - & - & - \\
\hline USGS-2A & - & - & - & & - & - & - & - & - & - & - & - & - \\
\hline MWV-01 & 0.16 & $4.4 \times 10^{-6}$ & $7.0 \times 10^{-9}$ & & - & - & - & - & - & - & - & - & - \\
\hline MWV-02 & - & - & - & & - & - & - & - & - & - & - & - . & - \\
\hline MWV-09 & - & - & - & - & 0.13 & $3.6 \times 10^{-6}$ & $5.7 \times 10^{-9}$ & 0.22 & $6.0 \times 10^{-6}$ & $9.6 \times 10^{-9}$ & - & - & - \\
\hline MWV-13 & - & - & - & & - & - & - & - & - & - & - & - & - \\
\hline MWV-16 & - & - & - & & - & - & - & - & - & - & - & - & - \\
\hline MWV-17 & - & - & - & & - & - & - & - & - & - & - & - & - \\
\hline MWV-18 & - & - & - & & - & - & - & - & - & - & - & - & - \\
\hline MWV-22 & - & - & - & & - & - & - & - & - & - & - & - & - \\
\hline MWV-24R & 0.36 & $9.9 \times 10^{-6}$ & $1.6 \times 10^{-8}$ & & - & - & - & 0.063 & $1.7 \times 10^{-6}$ & $2.8 \times 10^{-9}$ & - & - & - \\
\hline
\end{tabular}




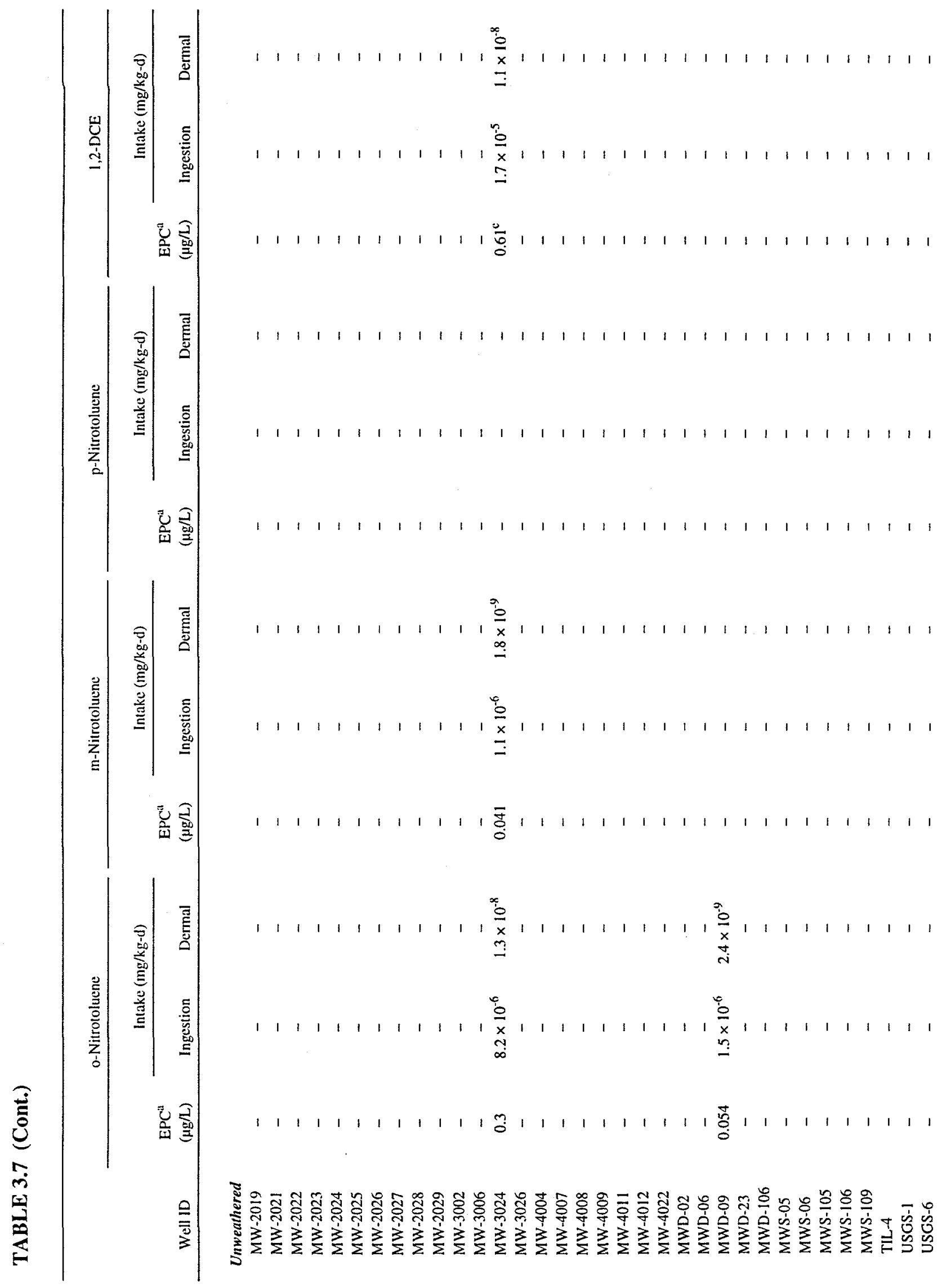


TABLE 3.7 (Cont.)

\begin{tabular}{|c|c|c|c|c|c|c|c|c|c|c|c|c|}
\hline \multirow[b]{3}{*}{ Well ID } & \multicolumn{3}{|c|}{ o-Nitrotoluene } & \multicolumn{3}{|c|}{ m-Nitrotoluene } & \multicolumn{3}{|c|}{ p-Nitrotoluene } & \multicolumn{3}{|c|}{ 1,2-DCE } \\
\hline & \multirow[b]{2}{*}{$\begin{array}{c}\mathrm{EPC}^{\mathrm{d}} \\
(\mu \mathrm{g} / \mathrm{L})\end{array}$} & \multicolumn{2}{|c|}{ Intake (mg/kg-d) } & \multirow[b]{2}{*}{$\begin{array}{c}\text { EPC }^{\mathrm{a}} \\
(\mu \mathrm{g} / \mathrm{L})\end{array}$} & \multicolumn{2}{|c|}{ Intake (mg/kg-d) } & \multirow[b]{2}{*}{$\begin{array}{c}\mathrm{EPC}^{\mathrm{a}} \\
(\mu \mathrm{g} / \mathrm{L})\end{array}$} & \multicolumn{2}{|c|}{ Intake (mg/kg-d) } & \multirow[b]{2}{*}{$\begin{array}{c}\mathrm{EPC}^{\mathrm{a}} \\
(\mu \mathrm{g} / \mathrm{L})\end{array}$} & \multicolumn{2}{|c|}{ Intake (mg/kg-d) } \\
\hline & & Ingestion & Dermal & & Ingestion & Dermal & & Ingestion & Dermal & & Ingestion & Dermal \\
\hline \multicolumn{13}{|l|}{ Weathered } \\
\hline MW-2001 & - & - & - & - & - & - & - & - & - & - & - & - \\
\hline MW-2002 & - & - & - & - & - & - & - & - & - & - & - & - \\
\hline MW-2003 & 0.18 & $4.9 \times 10^{-6}$ & $7.9 \times 10^{-9}$ & - & - & - & - & - & - & - & - & - \\
\hline MW-2004 & - & - & - & - & - & - & - & - & - & - & - & - \\
\hline MW-2005 & - & - & - & - & - & - & - & - & - & - & - & - \\
\hline MW-2006 & - & - & - & - & - & - & - & - & - & - & - & - \\
\hline MW-2007 & - & - & - & - & - & - & - & - & - & - & - & - \\
\hline MW-2008 & - & - & - & - & - & - & - & - & - & - & - & - \\
\hline MW-2009 & - & - & - & - & - & - & - & - & - & - & - & - \\
\hline$M W-2010$ & 0.083 & $2.3 \times 10^{-6}$ & $3.6 \times 10^{-9}$ & - & - & - & - & - & - & - & - & - \\
\hline$M W-2011$ & 0.22 & $6.0 \times 10^{-6}$ & $9.6 \times 10^{-9}$ & - & - & - & - & - & - & - & - & - \\
\hline$M W-2012$ & - & - & - & - & - & - & 0.28 & $7.7 \times 10^{-6}$ & $1.2 \times 10^{-8}$ & - & - & - \\
\hline$M W-2013$ & 0.26 & $7.1 \times 10^{-6}$ & $1.1 \times 10^{-8}$ & 0.058 & $1.6 \times 10^{-6}$ & $2.5 \times 10^{-9}$ & - & - & - & $16^{\mathrm{c}}$ & $4.4 \times 10^{-4}$ & $3.0 \times 10^{-7}$ \\
\hline MW-2014 & 0.22 & $6.0 \times 10^{-6}$ & $9.6 \times 10^{-9}$ & - & - & - & - & - & - & - & - & - \\
\hline$M W-2015$ & - & - & - & - & - & - & - & - & - & - & - & - \\
\hline$M W-2016$ & - & - & - & - & - & - & - & - & - & - & - & - \\
\hline$M W-2017$ & - & - & - & - & - & - & - & - & - & - & - & - \\
\hline MW-2018 & - & - & - & - & - & - & - & - & - & - & - & - \\
\hline MW-2020 & - & - & - & - & - & - & - & - & - & - & - & - \\
\hline MW-2030 & - & - & - & 0.06 & $1.6 \times 10^{-6}$ & $2.6 \times 10^{-9}$ & - & - & - & - & - & - \\
\hline MW-2034 & - & - & - & - & - & - & - & - & - & - & - & - \\
\hline MW-2035 & - & - & - & - & - & - & - & - & - & - & - & - \\
\hline MW-2036 & - & - & - & - & - & - & - & - & - & - & - & - \\
\hline MW-2037 & - & - & - & - & - & - & - & - & - & $22^{c}$ & $6.0 \times 10^{-4}$ & $4.1 \times 10^{-7}$ \\
\hline MW-2038 & 0.26 & $7.1 \times 10^{-6}$ & $1.1 \times 10^{-8}$ & - & - & - & - & - & - & $25^{\mathrm{C}}$ & $6.8 \times 10^{-4}$ & $4.6 \times 10^{-7}$ \\
\hline MW-2039 & 0.63 & $1.7 \times 10^{-5}$ & $2.8 \times 10^{-8}$ & - & - & - & - & - & - & - & - & - \\
\hline MW-2040 & - & - & - & - & - & - & - & - & - & - & - & - \\
\hline MW-2041 & - & - & - & - & - & - & - & - & - & - & - & - \\
\hline MW-2042 & - & - & - & - & - & - & - & - & - & - & - & - \\
\hline$M W-2043$ & - & - & - & - & - & - & - & - & - & - & - & - \\
\hline MW-2044 & - & - & - & - & - & - & - & - & - & - & - & - \\
\hline MW-2046 & - & - & - & - & - & - & - & - & - & $2^{c}$ & $5.5 \times 10^{-5}$ & $3.8 \times 10^{-8}$ \\
\hline MW-3003 & 0.5 & $1.4 \times 10^{-5}$ & $2.2 \times 10^{-8}$ & 0.14 & $3.8 \times 10^{-6}$ & $6.1 \times 10^{-9}$ & 0.15 & $4.1 \times 10^{-6}$ & $6.6 \times 10^{-9}$ & - & - & - \\
\hline MW-3007 & - & - & - & - & - & - & - & - & - & - & - & - \\
\hline
\end{tabular}




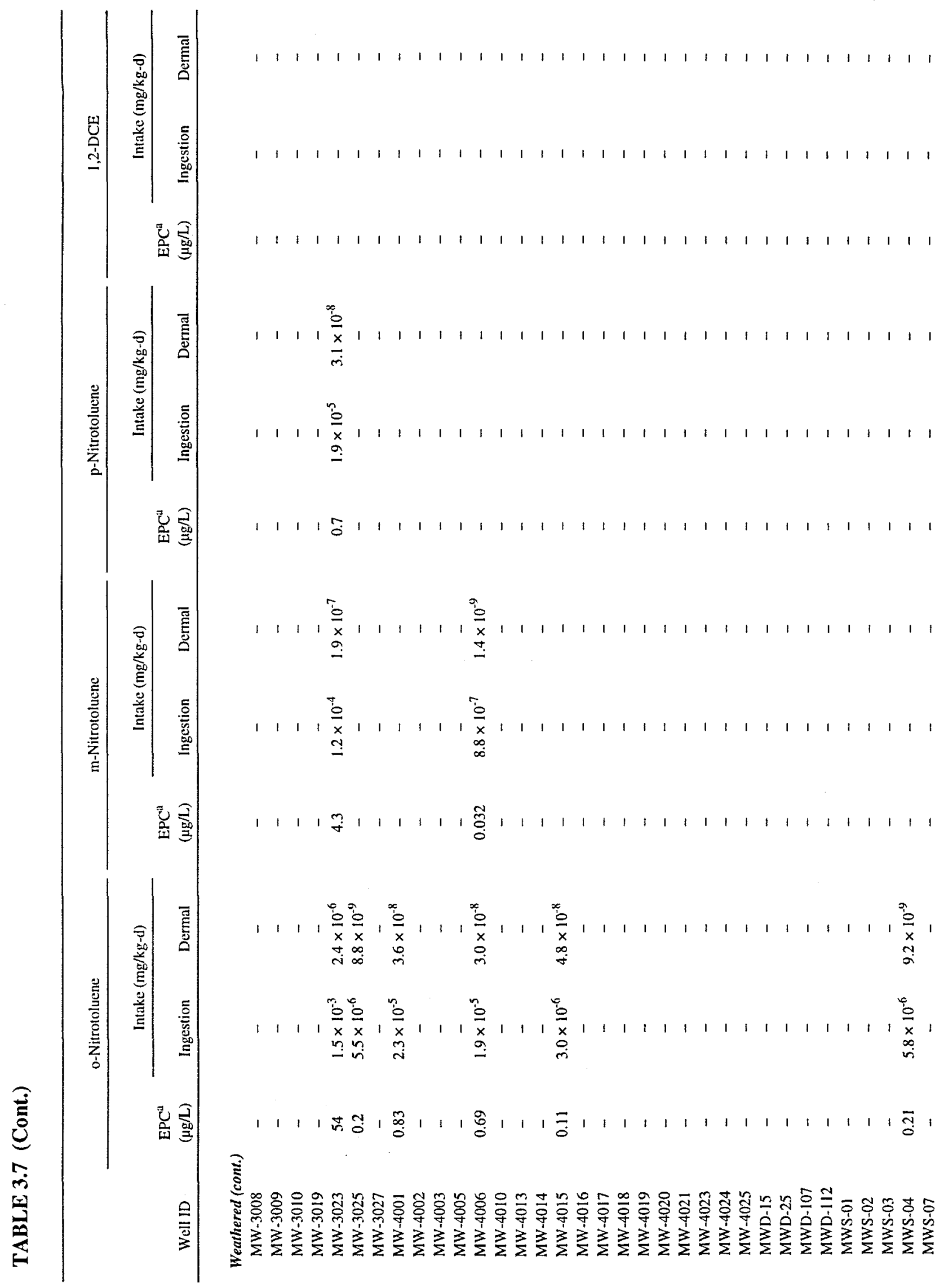


TABLE 3.7 (Cont.)

\begin{tabular}{|c|c|c|c|c|c|c|c|c|c|c|c|c|}
\hline \multirow[b]{3}{*}{ Well ID } & \multicolumn{3}{|c|}{ o-Nitrotoluene } & \multicolumn{3}{|c|}{ m-Nitrotoluene } & \multicolumn{3}{|c|}{ p-Nitrotoluene } & \multicolumn{3}{|c|}{ 1,2-DCE } \\
\hline & \multirow{2}{*}{$\begin{array}{c}\text { EPC }^{\mathrm{it}} \\
(\mu \mathrm{g} / \mathrm{L})\end{array}$} & \multicolumn{2}{|c|}{ Intake (mg/kg-d) } & \multirow{2}{*}{$\begin{array}{c}\mathrm{EPC}^{\mathrm{a}} \\
(\mu \mathrm{g} / \mathrm{L})\end{array}$} & \multicolumn{2}{|c|}{ Intake (mg/kg-d) } & \multirow{2}{*}{$\begin{array}{c}\mathrm{EPC}^{\mathrm{a}} \\
(\mu \mathrm{g} / \mathrm{L})\end{array}$} & \multicolumn{2}{|c|}{ Intake (mg/kg-d) } & \multirow{2}{*}{$\begin{array}{c}\mathrm{EPC}^{\mathrm{a}} \\
(\mu \mathrm{g} / \mathrm{L})\end{array}$} & \multicolumn{2}{|c|}{ Intake (mg/kg-d) } \\
\hline & & Ingestion & Dermal & & Ingestion & Dermal & & Ingestion & Dermal & & Ingestion & Dermal \\
\hline \multicolumn{13}{|c|}{ Weathered (cont.) } \\
\hline MWS-08 & - & - & - & - & - & - & - & - & - & - & - & - \\
\hline MWS-09 & - & - & - & - & - & - & - & - & - & - & - & - \\
\hline MWS-10 & 0.1 & $2.7 \times 10^{-6}$ & $4.4 \times 10^{-9}$ & - & - & - & - & - & - & - & - & - \\
\hline MWS-11 & - & - & - & - & - & - & - & - & - & - & - & - \\
\hline MWS-12 & 100 & $2.7 \times 10^{-3}$ & $4.4 \times 10^{-6}$ & 7.7 & $2.1 \times 10^{-4}$ & $3.4 \times 10^{-7}$ & 30 & $8.2 \times 10^{-4}$ & $1.3 \times 10^{-6}$ & - & - & - \\
\hline MWS-13 & - & - & - & - & - & - & - & - & - & - & - & - \\
\hline MWS-14 & - & - & - & - & - & - & - & - & - & - & - & - \\
\hline MWS-15 & - & - & - & - & - & - & - & - & - & - & - & - \\
\hline MWS-16 & 0.16 & $4.4 \times 10^{-6}$ & $7.0 \times 10^{-9}$ & - & - & - & - & - & - & - & - & - \\
\hline MWS- 17 & 8.8 & $2.4 \times 10^{-4}$ & $3.9 \times 10^{-7}$ & 0.31 & $8.5 \times 10^{-6}$ & $1.4 \times 10^{-8}$ & 0.93 & $2.5 \times 10^{-5}$ & $4.1 \times 10^{-8}$ & - & - & - \\
\hline MWS-19 & - & - & - & - & - & - & - & - & - & - & - & - \\
\hline MWS-20 & - & - & - & - & - & - & - & - & - & - & - & - \\
\hline MWS-21 & 0.15 & $4.1 \times 10^{-6}$ & $6.6 \times 10^{-9}$ & - & - & - & - & - & - & - & - & - \\
\hline MWS-22 & - & - & - & - & - & - & - & - & - & - & - & - \\
\hline MWS-24 & - & - & - & - & - & - & - & - & - & - & - & - \\
\hline MWS-25 & - & - & - & - & - & - & - & - & - & - & - & - \\
\hline MWS-26 & - & - & - & - & - & - & - & - & - & - & - & - \\
\hline MWS-104 & - & - & - & - & - & - & - & - & - & - & - & - \\
\hline MWS-107 & - & - & - & - & - & - & - & - & - & - & - & - \\
\hline MWS- 110 & - & - & - & - & - & - & - & - & - & - & - & - \\
\hline MWS- 112 & - & - & - & - & - & - & - & - & - & - & - & - \\
\hline USGS-2 & - & - & - & - & - & - & - & - & - & - & - & - \\
\hline USGS-3 & - & - & - & - & - & - & - & - & - & - & - & - \\
\hline USGS-4 & 1.0 & $2.7 \times 10^{-5}$ & $4.4 \times 10^{-8}$ & 0.11 & $3.0 \times 10^{-6}$ & $4.8 \times 10^{-9}$ & - & - & - & - & - & - \\
\hline USGS-5 & - & - & - & - & - & - & - & - & - & - & - & - \\
\hline USGS-7 & - & - & - & - & - & - & - & - & - & - & - & - \\
\hline USGS-8 & - & - & - & - & - & - & - & - & - & - & - & - \\
\hline USGS-9 & - & - & - & - & - & - & - & - & - & - & - & - \\
\hline
\end{tabular}

a EPC values are the maximum concentrations reported from the 1995 joint DOE/DA sampling rounds or the maximum 95\% UCL from 1996-1997 (indicated by footnote c).

b A hyphen $(-)$ indicates the parameter was not detected.

c EPC values are the maximum 95\% UCL reported from samples collected in 1996-1997. 
TABLE 3.8 Estimated Carcinogenic Intakes of Organic Compound COPCs for the Hypothetical Future Resident

\begin{tabular}{|c|c|c|c|c|c|c|c|c|c|c|c|c|}
\hline \multirow[b]{3}{*}{ Well ID } & \multicolumn{3}{|c|}{ 2,4,6-TNT } & \multicolumn{3}{|c|}{ 2,4-DNT } & \multicolumn{3}{|c|}{ 2,6-DNT } & \multicolumn{3}{|c|}{$\mathrm{TCE}^{\mathrm{b}}$} \\
\hline & \multirow[b]{2}{*}{$\begin{array}{c}\mathrm{EPC}^{\mathrm{a}} \\
(\mu \mathrm{g} / \mathrm{L})\end{array}$} & \multicolumn{2}{|c|}{ Intake (mg/kg-d) } & \multirow[b]{2}{*}{$\begin{array}{l}E^{E P C} \\
(\mu \mathrm{g} / \mathrm{L})\end{array}$} & \multicolumn{2}{|c|}{ Intake (mg/kg-d) } & \multirow[b]{2}{*}{$\begin{array}{l}\mathrm{EPC}^{\mathrm{a}} \\
(\mu \mathrm{g} / \mathrm{L})\end{array}$} & \multicolumn{2}{|c|}{ Intake (mg/kg-d) } & \multirow[b]{2}{*}{$\begin{array}{c}\mathrm{EPC}^{\mathrm{a}} \\
(\mu \mathrm{g} / \mathrm{L})\end{array}$} & \multicolumn{2}{|c|}{ Intake ( $\mathrm{mg} / \mathrm{kg}-\mathrm{d})$} \\
\hline & & Ingestion & Dermal & & Ingestion & Dermal & & Ingestion & Dermal & & Ingestion & Dermal \\
\hline \multicolumn{13}{|l|}{ Deep Wells } \\
\hline MWD-05 & $-c$ & - & - & - & - & - & - & - & - & - & - & - \\
\hline MWD-18 & - & - & - & - & - & - & - & - & - & - & - & - \\
\hline MWGS-01 & - & - & - & - & - & - & - & - & - & - & - & - \\
\hline MWGS-02 & - & - & - & - & - & - & - & - & - & - & - & - \\
\hline MWS-18 & - & - & - & - & - & - & - & - & - & - & - & - \\
\hline MWS-101 & - & - & - & - & - & - & - & - & - & - & - & - \\
\hline MWS-102 & - & - & - & - & - & - & - & - & - & - & - & - \\
\hline MWS-103 & - & - & - & - & - & - & - & - & - & - & - & - \\
\hline TIL-3 & - & - & - & - & - & - & - & - & - & - & - & - \\
\hline \multicolumn{13}{|l|}{ Overburden } \\
\hline MW-2031 & - & - & - & - & - & - & - & - & - & - & - & - \\
\hline$M W-2032$ & 6.7 & $7.9 \times 10^{-5}$ & $1.3 \times 10^{-7}$ & 0.14 & $1.6 \times 10^{-6}$ & $2.6 \times 10^{-9}$ & 4.4 & $5.2 \times 10^{-5}$ & $8.3 \times 10^{-8}$ & 0.63 & $\begin{array}{c}7.4 \times 10^{-6} \\
\left(2.0 \times 10^{-5}\right)\end{array}$ & $1.9 \times 10^{-8}$ \\
\hline MW-2033 & 1.2 & $1.4 \times 10^{-5}$ & $2.3 \times 10^{-8}$ & 0.55 & $6.5 \times 10^{-6}$ & $1.0 \times 10^{-8}$ & 4.9 & $5.8 \times 10^{-5}$ & $9.2 \times 10^{-8}$ & - & - & - \\
\hline MW-300I & - & - & - & - & - & - & - & - & - & - & - & - \\
\hline$M W-3013$ & - & - & - & - & - & - & - & - & - & - & - & - \\
\hline MW-3018 & - & - & - & - & - & - & - & - & - & - & - & - \\
\hline MW-3022 & - & - & - & - & - & - & - & - & - & - & - & - \\
\hline USGS-2A & - & - & - & - & - & - & - & - & - & - & - & - \\
\hline MWV-01 & 11 & $1.3 \times 10^{-4}$ & $2.1 \times 10^{-7}$ & 0.11 & $1.3 \times 10^{-6}$ & $2.1 \times 10^{-9}$ & 1.0 & $1.2 \times 10^{-5}$ & $1.9 \times 10^{-8}$ & - & - & - \\
\hline MWV-02 & 0.11 & $1.3 \times 10^{-6}$ & $2.1 \times 10^{-9}$ & 0.059 & $6.9 \times 10^{-7}$ & $1.1 \times 10^{-9}$ & 0.048 & $5.6 \times 10^{-7}$ & $9.0 \times 10^{-10}$ & - & - & - \\
\hline MWV-09 & 30 & $3.5 \times 10^{-4}$ & $5.6 \times 10^{-7}$ & 20 & $2.3 \times 10^{-4}$ & $3.8 \times 10^{-7}$ & 2.9 & $3.4 \times 10^{-5}$ & $5.4 \times 10^{-8}$ & - & - & - \\
\hline MWV-13 & - & - & - & - & - & - & - & - & - & - & - & - \\
\hline MWV-16 & 0.27 & $3.2 \times 10^{-6}$ & $5.1 \times 10^{-9}$ & - & - & - & 0.069 & $8.1 \times 10^{-7}$ & $1.3 \times 10^{-9}$ & - & - & - \\
\hline MWV-17 & - & - & - & - & - & - & - & - & - & - & - & - \\
\hline MWV-18 & - & - & - & - & - & - & - & - & - & - & - & - \\
\hline MWV-22 & - & - & - & - & - & - & 0.14 & $1.6 \times 10^{-6}$ & $2.6 \times 10^{-9}$ & - & - & - \\
\hline$M W V-24 R$ & 1.1 & $1.3 \times 10^{-5}$ & $2.1 \times 10^{-8}$ & 0.13 & $1.5 \times 10^{-6}$ & $2.4 \times 10^{-9}$ & 1.4 & $1.6 \times 10^{-5}$ & $2.6 \times 10^{-8}$ & - & - & - \\
\hline
\end{tabular}


TABLE 3.8 (Cont.)

\begin{tabular}{|c|c|c|c|c|c|c|c|c|c|c|c|c|}
\hline \multirow[b]{3}{*}{ Well ID } & \multicolumn{3}{|c|}{$2,4,6-\mathrm{TNT}$} & \multicolumn{3}{|c|}{ 2,4-DNT } & \multicolumn{3}{|c|}{ 2,6-DNT } & \multicolumn{3}{|c|}{$\mathrm{TCE}^{\mathrm{h}}$} \\
\hline & \multirow{2}{*}{$\begin{array}{l}\mathrm{EPC}^{\mathrm{a}} \\
(\mu \mathrm{g} / \mathrm{L})\end{array}$} & \multicolumn{2}{|c|}{ Intake (mg/kg-d) } & \multirow{2}{*}{$\begin{array}{c}\mathrm{EPC}^{\mathrm{a}} \\
(\mu \mathrm{g} / \mathrm{L})\end{array}$} & \multicolumn{2}{|c|}{ Intake (mg/kg-d) } & \multirow{2}{*}{$\begin{array}{c}\mathrm{EPC}^{\mathrm{a}} \\
(\mu \mathrm{g} / \mathrm{L})\end{array}$} & \multicolumn{2}{|c|}{ Intake (mg/kg-d) } & \multirow{2}{*}{$\begin{array}{l}\text { EPC }^{a} \\
(\mu g / L)\end{array}$} & \multicolumn{2}{|c|}{ Intake (mg/kg-d) } \\
\hline & & Ingestion & Dermal & & Ingestion & Dermal & & Ingestion & Dermal & & Ingestion & Dermal \\
\hline \multicolumn{13}{|l|}{ Unweathered } \\
\hline MW-2019 & - & - & - & - & - & - & - & - & - & - & - & - \\
\hline MW-2021 & - & - & - & - & - & - & - & - & - & - & - & - \\
\hline MW-2022 & - & - & - & - & - & - & - & - & - & - & - & - \\
\hline MW-2023 & - & - & - & - & - & - & - & - & - & - & - & - \\
\hline MW-2024 & - & - & - & - & - & - & - & - & - & - & - & - \\
\hline MW-2025 & - & - & - & - & - & - & - & - & - & - & - & - \\
\hline MW-2026 & - & - & - & - & - & - & - & - & - & - & - & - \\
\hline$M W-2027$ & - & - & - & - & - & - & - & - & - & - & - & - \\
\hline MW-2028 & - & - & - & - & - & - & - & - & - & - & - & - \\
\hline MW-2029 & - & - & - & - & - & - & - & - & - & - & - & - \\
\hline MW-3002 & - & - & - & - & - & - & - & - & - & - & - & - \\
\hline MW-3006 & - & - & - & - & - & - & - & - & - & - & - & - \\
\hline MW-3024 & - & - & - & 0.13 & $1.5 \times 10^{-6}$ & $2.4 \times 10^{-9}$ & 0.45 & $5.3 \times 10^{-6}$ & $8.5 \times 10^{-9}$ & 50 & $\begin{array}{c}5.9 \times 10^{-4} \\
\left(1.6 \times 10^{-3}\right)\end{array}$ & $1.5 \times 10^{-6}$ \\
\hline MW-3026 & - & - & - & 0.072 & $8.5 \times 10^{-7}$ & $1.4 \times 10^{-9}$ & 0.046 & $5.4 \times 10^{-7}$ & $8.6 \times 10^{-10}$ & - & - & - \\
\hline MW-4004 & - & - & - & - & - & - & - & - & - & - & - & - \\
\hline MW-4007 & - & - & - & - & - & - & - & - & - & - & - & - \\
\hline MW-4008 & - & - & - & - & - & - & - & - & - & - & - & - \\
\hline MW-4009 & - & - & - & - & - & - & - & - & - & - & - & - \\
\hline$M W-4011$ & - & - & - & - & - & - & 0.065 & $7.6 \times 10^{-7}$ & $1.2 \times 10^{-9}$ & - & - & - \\
\hline MW-4012 & - & - & - & - & - & - & - & - & - & - & - & - \\
\hline MW-4022 & - & - & - & - & - & - & - & - & - & - & - & - \\
\hline MWD-02 & - & - & - & - & - & - & - & - & - & - & - & - \\
\hline MWD-06 & - & - & - & - & - & - & - & - & - & - & - & - \\
\hline MWD-09 & - & - & - & 0.042 & $4.9 \times 10^{-7}$ & $7.9 \times 10^{-10}$ & 0.16 & $1.9 \times 10^{-6}$ & $3.0 \times 10^{-9}$ & - & - & - \\
\hline MWD-23 & - & - & - & - & - & - & - & - & - & - & - & - \\
\hline MWD-106 & - & - & - & - & - & - & - & - & - & - & - & - \\
\hline MWS-05 & - & - & - & - & - & - & - & - & - & - & - & - \\
\hline MWS-06 & - & - & - & - & - & - & - & - & - & - & - & - \\
\hline MWS- 105 & - & - & - & - & - & - & - & - & - & - & - & - \\
\hline MWS-106 & - & - & - & - & - & - & - & - & - & - & - & - \\
\hline MWS-109 & - & - & - & - & - & - & - & - & - & - & - & - \\
\hline TIL-4 & - & - & - & - & - & - & - & - & - & - & - & - \\
\hline USGS-1 & - & - & - & 0.051 & $6.0 \times 10^{-7}$ & $9.6 \times 10^{-10}$ & 0.022 & $2.6 \times 10^{-7}$ & $4.1 \times 10^{-10}$ & - & - & - \\
\hline USGS-6 & - & - & - & - & - & - & - & - & - & - & - & - \\
\hline
\end{tabular}


TABLE 3.8 (Cont.)

\begin{tabular}{|c|c|c|c|c|c|c|c|c|c|c|c|c|}
\hline \multirow[b]{3}{*}{ Well ID } & \multicolumn{3}{|c|}{ 2,4,6-TNT } & \multicolumn{3}{|c|}{ 2,4-DNT } & \multicolumn{3}{|c|}{ 2,6-DNT } & \multicolumn{3}{|c|}{$\mathrm{TCE}^{\mathrm{b}}$} \\
\hline & \multirow{2}{*}{$\begin{array}{c}\mathrm{EPC}^{\mathrm{a}} \\
(\mu \mathrm{g} / \mathrm{L})\end{array}$} & \multicolumn{2}{|c|}{ Intake (mg/kg-d) } & \multirow{2}{*}{$\begin{array}{c}\mathrm{EPC}^{\mathrm{a}} \\
(\mu \mathrm{g} / \mathrm{L})\end{array}$} & \multicolumn{2}{|c|}{ Intake (mg/kg-d) } & \multirow{2}{*}{$\begin{array}{c}\mathrm{EPC}^{\mathrm{a}} \\
(\mu \mathrm{g} / \mathrm{L})\end{array}$} & \multicolumn{2}{|c|}{ Intake (mg/kg-d) } & \multirow{2}{*}{$\begin{array}{c}\mathrm{EPC}^{\mathrm{a}} \\
(\mu \mathrm{g} / \mathrm{L})\end{array}$} & \multicolumn{2}{|c|}{ Intake (mg/kg-d) } \\
\hline & & Ingestion & Dermal & & Ingestion & Dermal & & Ingestion & Dermal & & Ingestion & Dermal \\
\hline \multicolumn{13}{|l|}{ Weathered } \\
\hline MW-200I & - & - & - & 0.13 & $1.5 \times 10^{-6}$ & $2.4 \times 10^{-9}$ & 0.056 & $6.6 \times 10^{-7}$ & $1.1 \times 10^{-9}$ & - & - & - \\
\hline MW-2002 & - & - & - & 0.07 & $8.2 \times 10^{-7}$ & $1.3 \times 10^{-9}$ & 0.41 & $4.8 \times 10^{-6}$ & $7.7 \times 10^{-9}$ & - & - & - \\
\hline MW-2003 & - & - & - & 0.15 & $1.8 \times 10^{-6}$ & $2.8 \times 10^{-9}$ & 0.45 & $5.3 \times 10^{-6}$ & $8.5 \times 10^{-9}$ & - & - & - \\
\hline MW-2004 & - & - & - & - & - & - & - & - & - & - & - & - \\
\hline MW-2005 & - & - & - & 0.061 & $7.2 \times 10^{-7}$ & $1.1 \times 10^{-9}$ & 0.090 & $1.1 \times 10^{-6}$ & $1.7 \times 10^{-9}$ & - & - & - \\
\hline MW-2006 & - & - & - & 0.14 & $1.6 \times 10^{-6}$ & $2.6 \times 10^{-9}$ & 0.0090 & $1.1 \times 10^{-7}$ & $1.7 \times 10^{-10}$ & - & - & - \\
\hline MW-2007 & - & - & - & - & - & - & - & - & - & - & - & - \\
\hline$M W-2008$ & - & - & - & - & - & - & - & - & - & - & - & - \\
\hline MW-2009 & - & - & - & - & - & - & - & - & - & - & - & - \\
\hline$M W-2010$ & 0.34 & $4.0 \times 10^{-6}$ & $6.4 \times 10^{-9}$ & 0.094 & $1.1 \times 10^{-6}$ & $1.8 \times 10^{-9}$ & 0.75 & $8.8 \times 10^{-6}$ & $1.4 \times 10^{-8}$ & - & - & - \\
\hline MW-2011 & - & - & - & 0.20 & $2.3 \times 10^{-6}$ & $3.8 \times 10^{-9}$ & 1.6 & $1.9 \times 10^{-5}$ & $3.0 \times 10^{-8}$ & - & - & - \\
\hline$M W-2012$ & 0.46 & $5.4 \times 10^{-6}$ & $8.6 \times 10^{-9}$ & 0.099 & $1.2 \times 10^{-6}$ & $1.9 \times 10^{-9}$ & 0.65 & $7.6 \times 10^{-6}$ & $1.2 \times 10^{-8}$ & - & - & - \\
\hline MW-2013 & 0.85 & $1.0 \times 10^{-5}$ & $1.6 \times 10^{-8}$ & 0.36 & $4.2 \times 10^{-6}$ & $6.8 \times 10^{-9}$ & 4.4 & $5.2 \times 10^{-5}$ & $8.3 \times 10^{-8}$ & 2.0 & $\begin{array}{c}2.3 \times 10^{-5} \\
\left(6.5 \times 10^{-5}\right)\end{array}$ & $6.0 \times 10^{-8}$ \\
\hline$M W-2014$ & 0.044 & $5.2 \times 10^{-7}$ & $8.3 \times 10^{-10}$ & 0.16 & $1.9 \times 10^{-6}$ & $3.0 \times 10^{-9}$ & 0.41 & $4.8 \times 10^{-6}$ & $7.7 \times 10^{-9}$ & - & - & - \\
\hline$M W-2015$ & - & - & - & - & - & - & - & - & - & - & - & - \\
\hline MW-2016 & - & - & - & - & - & - & - & - & - & - & - & - \\
\hline$M W-2017$ & - & - & - & - & - & - & - & - & - & - & - & - \\
\hline MW-2018 & - & - & - & - & - & - & - & - & - & - & - & - \\
\hline MW-2020 & - & - & - & - & - & - & - & - & - & - & - & - \\
\hline$M W-2030$ & 29 & $3.4 \times 10^{-4}$ & $5.4 \times 10^{-7}$ & 0.25 & $2.9 \times 10^{-6}$ & $4.7 \times 10^{-9}$ & 11 & $1.3 \times 10^{-4}$ & $2.1 \times 10^{-7}$ & - & - & - \\
\hline MW-2034 & - & - & - & - & - & - & - & - & - & - & - & - \\
\hline MW-2035 & - & - & - & - & - & - & - & - & - & - & - & - \\
\hline MW-2036 & - & - & - & - & - & - & - & - & - & & & \\
\hline MW-2037 & - & - & - & 0.56 & $6.6 \times 10^{-6}$ & $1.1 \times 10^{-8}$ & 0.13 & $1.5 \times 10^{-6}$ & $2.4 \times 10^{-9}$ & 1,200 & $\begin{array}{r}1.5 \times 10^{-2} \\
\left(4.0 \times 10^{-2}\right)\end{array}$ & $3.7 \times 10^{-5}$ \\
\hline MW-2038 & - & - & - & 1.7 & $2.0 \times 10^{-5}$ & $3.2 \times 10^{-8}$ & 0.32 & $3.8 \times 10^{-6}$ & $6.0 \times 10^{-9}$ & 3,800 & $\begin{array}{c}4.5 \times 10^{-2} \\
\left(1.2 \times 10^{-1}\right)\end{array}$ & $1.1 \times 10^{-4}$ \\
\hline MW-2039 & - & - & - & 0.12 & $1.4 \times 10^{-6}$ & $2.3 \times 10^{-9}$ & 1.7 & $2.0 \times 10^{-5}$ & $3.2 \times 10^{-8}$ & - & - & - \\
\hline MW-2040 & - & - & - & - & - & - & - & _- & - & - & - & - \\
\hline MW-204I & - & - & - & - & - & - & - & - & - & - & - & - \\
\hline$M W-2042$ & - & - & - & - & - & - & - & - & - & - & - & - \\
\hline$M W-2043$ & - & - & - & 0.087 & $1.0 \times 10^{-6}$ & $1.6 \times 10^{-9}$ & - & - & - & - & - & - \\
\hline MW-2044 & - & - & - & - & - & - & - & - & - & - & - & - \\
\hline MW-3003 & - & - & - & 0.17 & $2.0 \times 10^{-6}$ & $3.2 \times 10^{-9}$ & 0.085 & $1.0 \times 10^{-6}$ & $1.6 \times 10^{-9}$ & - & - & - \\
\hline
\end{tabular}


TABLE 3.8 (Cont.)

\begin{tabular}{|c|c|c|c|c|c|c|c|c|c|c|c|c|}
\hline \multirow[b]{3}{*}{ Well ID } & \multicolumn{3}{|c|}{ 2,4,6-TNT } & \multicolumn{3}{|c|}{ 2,4-DNT } & \multicolumn{3}{|c|}{ 2,6-DNT } & \multicolumn{3}{|c|}{$\mathrm{TCE}^{\mathrm{b}}$} \\
\hline & \multirow[b]{2}{*}{$\begin{array}{l}\text { EPC } \\
(\mu \mathrm{g} / \mathrm{L}) \\
\end{array}$} & \multicolumn{2}{|c|}{ Intake (mg/kg-d) } & \multirow[b]{2}{*}{$\begin{array}{c}E^{E P C}{ }^{\mathrm{a}} \\
(\mu \mathrm{g} / \mathrm{L})\end{array}$} & \multicolumn{2}{|c|}{ Intake (mg/kg-d) } & \multirow[b]{2}{*}{$\begin{array}{l}\mathrm{EPC}^{\mathrm{a}} \\
(\mu \mathrm{g} / \mathrm{L})\end{array}$} & \multicolumn{2}{|c|}{ Intake (mg/kg-d) } & \multirow[b]{2}{*}{$\begin{array}{l}\mathrm{EPC}^{\mathrm{a}} \\
(\mu \mathrm{g} / \mathrm{L})\end{array}$} & \multicolumn{2}{|c|}{ Intake (mg/kg-d) } \\
\hline & & Ingestion & Dermal & & Ingestion & Dermal & & Ingestion & Dermal & & Ingestion & Dermal \\
\hline \multicolumn{13}{|c|}{ Weathered (cont.) } \\
\hline MW-3007 & - & - & - & - & - & - & - & - & - & - & - & - \\
\hline MW-3008 & - & - & - & - & - & - & - & - & - & - & - & - \\
\hline MW-3009 & - & - & - & - & - & - & - & - & - & - & - & - \\
\hline MW-3010 & - & - & - & - & - & - & - & - & - & - & - & - \\
\hline MW-3019 & - & - & - & - & - & 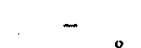 & - & - & - & - & - & - \\
\hline MW-3023 & - & - & - & 5.0 & $5.9 \times 10^{-5}$ & $9.4 \times 10^{-8}$ & 5.0 & $5.9 \times 10^{-5}$ & $9.4 \times 10^{-8}$ & - & - & - \\
\hline MW-3025 & - & - & - & 0.094 & $1.1 \times 10^{-6}$ & $1.8 \times 10^{-9}$ & - & - & - & 36 & $\begin{array}{c}4.3 \times 10^{-4} \\
\left(1.2 \times 10^{-3}\right)\end{array}$ & $1.1 \times 10^{-6}$ \\
\hline MW-3027 & - & - & - & 0.058 & $6.8 \times 10^{-7}$ & $1.1 \times 10^{-9}$ & 0.040 & $4.7 \times 10^{-7}$ & $7.5 \times 10^{-10}$ & 2.7 & $\begin{array}{c}3.1 \times 10^{-5} \\
\left(8.7 \times 10^{-5}\right)\end{array}$ & $8.0 \times 10^{-8}$ \\
\hline MW-400I & 1.8 & $2.1 \times 10^{-5}$ & $3.4 \times 10^{-8}$ & 1.3 & $1.5 \times 10^{-5}$ & $2.4 \times 10^{-8}$ & 3.1 & $3.6 \times 10^{-5}$ & $5.8 \times 10^{-8}$ & 4.0 & $\begin{array}{c}4.7 \times 10^{-5} \\
\left(1.3 \times 10^{-4}\right)\end{array}$ & $1.2 \times 10^{-7}$ \\
\hline$M W-4002$ & 1.8 & $2.1 \times 10^{-5}$ & $3.4 \times 10^{-8}$ & 0.14 & $1.6 \times 10^{-6}$ & $2.6 \times 10^{-9}$ & 0.29 & $3.4 \times 10^{-6}$ & $5.4 \times 10^{-9}$ & - & - & - \\
\hline MW-4003 & - & - & - & - & - & - & - & - & - & - & - & - \\
\hline$M W-4005$ & - & - & - & - & - & - & - & - & - & - & - & - \\
\hline MW-4006 & - & - & - & 0.16 & $1.9 \times 10^{-6}$ & $3.0 \times 10^{-9}$ & 3.1 & $3.6 \times 10^{-5}$ & $5.8 \times 10^{-8}$ & - & - & - \\
\hline MW-4010 & - & - & - & - & - & - & - & - & - & - & - & - \\
\hline$M W-4013$ & 0.046 & $5.4 \times 10^{-7}$ & $8.6 \times 10^{-10}$ & 0.077 & $9.0 \times 10^{-7}$ & $1.4 \times 10^{-9}$ & 0.74 & $8.7 \times 10^{-6}$ & $1.4 \times 10^{-8}$ & - & - & - \\
\hline MW-4014 & - & - & - & 0.026 & $3.1 \times 10^{-7}$ & $4.9 \times 10^{-10}$ & 0.087 & $1.0 \times 10^{-6}$ & $1.6 \times 10^{-9}$ & - & - & - \\
\hline MW-4015 & - & - & - & 0.19 & $2.2 \times 10^{-6}$ & $3.6 \times 10^{-9}$ & 1.1 & $1.3 \times 10^{-5}$ & $2.1 \times 10^{-8}$ & - & - & - \\
\hline MW-4016 & - & - & - & - & - & - & - & - & - & - & - & - \\
\hline$M W-4017$ & - & - & - & - & - & - & - & - & - & - & - & - \\
\hline MW-4018 & - & - & - & - & - & - & - & - & - & - & - & - \\
\hline MW-4019 & - & - & - & - & - & - & - & - & - & - & - & - \\
\hline MW-4020 & - & - & - & - & - & - & - & - & - & - & - & - \\
\hline$M W-4021$ & - & - & - & - & - & - & - & - & - & - & - & - \\
\hline$M W-4023$ & - & - & - & 0.067 & $7.9 \times 10^{-7}$ & $1.3 \times 10^{-9}$ & 0.023 & $2.7 \times 10^{-7}$ & $4.3 \times 10^{-10}$ & - & - & - \\
\hline MW-4024 & - & - & - & - & - & - & - & - & - & - & - & - \\
\hline MW-4025 & - & - & - & - & - & - & - & - & - & - & - & - \\
\hline MWD-15 & - & - & - & - & - & - & 0.64 & $7.5 \times 10^{-6}$ & $1.2 \times 10^{-8}$ & - & - & - \\
\hline MWD-25 & - & - & - & - & - & - & - & - & - & - & - & - \\
\hline MWD-107 & - & - & - & 0.020 & $2.3 \times 10^{-7}$ & $3.8 \times 10^{-10}$ & 0.090 & $1.1 \times 10^{-6}$ & $1.7 \times 10^{-9}$ & - & - & - \\
\hline MWD-112 & - & - & - & - & - & - & - & - & - & - & - & - \\
\hline MWS-01 & - & - & - & - & - & - & 0.64 & $7.5 \times 10^{-6}$ & $1.2 \times 10^{-8}$ & - & - & - \\
\hline MWS-02 & - & - & - & - & - & - & - & - & - & - & - & - \\
\hline MWS-03 & - & - & - & - & - & - & - & - & - & - & - & - \\
\hline
\end{tabular}


TABLE 3.8 (Cont.)

\begin{tabular}{|c|c|c|c|c|c|c|c|c|c|c|c|c|}
\hline \multirow[b]{3}{*}{ Well ID } & \multicolumn{3}{|c|}{ 2,4,6-TNT } & \multicolumn{3}{|c|}{ 2,4-DNT } & \multicolumn{3}{|c|}{ 2,6-DNT } & \multicolumn{3}{|c|}{$\mathrm{TCE}^{\mathrm{b}}$} \\
\hline & \multirow[b]{2}{*}{$\begin{array}{l}\mathrm{EPC}^{\mathrm{a}} \\
(\mu \mathrm{g} / \mathrm{L})\end{array}$} & \multicolumn{2}{|c|}{ Intake (mg/kg-d) } & \multirow[b]{2}{*}{$\begin{array}{l}\mathrm{EPC}^{\mathrm{a}} \\
(\mu \mathrm{g} / \mathrm{L})\end{array}$} & \multicolumn{2}{|c|}{ Intake (mg/kg-d) } & \multirow[b]{2}{*}{$\begin{array}{l}E^{E P C} C^{i} \\
(\mu \mathrm{g} / \mathrm{L})\end{array}$} & \multicolumn{2}{|c|}{ Intake (mg/kg-d) } & \multirow[b]{2}{*}{$\begin{array}{c}\mathrm{EPC}^{\mathrm{a}} \\
(\mu \mathrm{g} / \mathrm{L})\end{array}$} & \multicolumn{2}{|c|}{ Intake (mg/kg-d) } \\
\hline & & Ingestion & Dermal & & Ingestion & Dermal & & Ingestion & Dermal & & Ingestion & Dermal \\
\hline \multicolumn{13}{|c|}{ Weathered (cont.) } \\
\hline MWS-04 & 1.2 & $1.4 \times 10^{-5}$ & $2.3 \times 10^{-8}$ & 0.10 & $1.2 \times 10^{-6}$ & $1.9 \times 10^{-9}$ & 1.2 & $1.4 \times 10^{-5}$ & $2.3 \times 10^{-8}$ & - & - & - \\
\hline MWS:07 & 2.6 & $3.1 \times 10^{-5}$ & $4.9 \times 10^{-8}$ & 0.049 & $5.8 \times 10^{-7}$ & $9.2 \times 10^{-10}$ & 1.2 & $1.4 \times 10^{-5}$ & $2.3 \times 10^{-8}$ & - & - & - \\
\hline MWS-08 & - & - & - & - & - & - & - & - & - & - & - & - \\
\hline MWS-09 & - & - & - & - & - & - & - & - & - & - & - & - \\
\hline MWS-10 & 0.028 & $3.3 \times 10^{-7}$ & $5.3 \times 10^{-10}$ & 0.082 & $9.6 \times 10^{-7}$ & $1.5 \times 10^{-9}$ & 2.0 & $2.3 \times 10^{-5}$ & $3.8 \times 10^{-8}$ & - & - & - \\
\hline MWS-11 & 0.046 & $5.4 \times 10^{-7}$ & $8.6 \times 10^{-10}$ & 0.055 & $6.5 \times 10^{-7}$ & $1.0 \times 10^{-9}$ & 0.54 & $6.3 \times 10^{-6}$ & $1.0 \times 10^{-8}$ & - & - & - \\
\hline MWS-12 & 0.18 & $2.1 \times 10^{-6}$ & $3.4 \times 10^{-9}$ & 8.8 & $1.0 \times 10^{-4}$ & $1.7 \times 10^{-7}$ & 15 & $1.8 \times 10^{-4}$ & $2.8 \times 10^{-7}$ & - & - & - \\
\hline MWS-13 & - & - & - & - & - & - & - & - & - & - & - & - \\
\hline MWS-14 & - & - & & - & - & - & - & - & - & - & - & - \\
\hline MWS-15 & 5.9 & $6.9 \times 10^{-5}$ & $1.1 \times 10^{-7}$ & 0.081 & $9.5 \times 10^{-7}$ & $1.5 \times 10^{-9}$ & 1.0 & $1.2 \times 10^{-5}$ & $1.9 \times 10^{-8}$ & - & - & - \\
\hline MWS-16 & 2.9 & $3.4 \times 10^{-5}$ & $5.4 \times 10^{-8}$ & 0.092 & $1.1 \times 10^{-6}$ & $1.7 \times 10^{-9}$ & 1.2 & $1.4 \times 10^{-5}$ & $2.3 \times 10^{-8}$ & - & - & - \\
\hline MWS-17 & 0.15 & $1.8 \times 10^{-6}$ & $2.8 \times 10^{-9}$ & 1.1 & $1.3 \times 10^{-5}$ & $2.1 \times 10^{-8}$ & 13 & $1.5 \times 10^{-4}$ & $2.4 \times 10^{-7}$ & - & - & - \\
\hline MWS-19 & - & - & - & 0.08 & $9.4 \times 10^{-7}$ & $1.5 \times 10^{-9}$ & 0.14 & $1.6 \times 10^{-6}$ & $2.6 \times 10^{-9}$ & - & - & - \\
\hline MWS-20 & - & - & - & - & - & - & 0.013 & $1.5 \times 10^{-7}$ & $2.4 \times 10^{-10}$ & - & - & - \\
\hline MWS-21 & - & - & - & 0.94 & $1.1 \times 10^{-5}$ & $1.8 \times 10^{-8}$ & 0.17 & $2.0 \times 10^{-6}$ & $3.2 \times 10^{-9}$ & 800 & $\begin{array}{c}9.4 \times 10^{-3} \\
\left(2.6 \times 10^{-2}\right)\end{array}$ & $2.4 \times 10^{-5}$ \\
\hline MWS-22 & - & - & - & 0.025 & $2.9 \times 10^{-7}$ & $4.7 \times 10^{-10}$ & 0.13 & $1.5 \times 10^{-6}$ & $2.4 \times 10^{-9}$ & - & - & - \\
\hline MWS-24 & - & - & - & - & - & - & - & - & - & - & - & - \\
\hline MWS-25 & - & - & - & - & - & - & - & - & - & - & - & - \\
\hline MWS-26 & - & - & - & - & - & - & - & - & - & - & - & - \\
\hline MWS-104 & - & - & - & - & - & - & - & - & - & - & - & - \\
\hline MWS-107 & - & - & - & 0.059 & $6.9 \times 10^{-7}$ & $1.1 \times 10^{-9}$ & 0.16 & $1.9 \times 10^{-6}$ & $3.0 \times 10^{-9}$ & - & - & - \\
\hline MWS-110 & - & - & - & - & - & - & 0.054 & $6.3 \times 10^{-7}$ & $1.0 \times 10^{-9}$ & - & - & - \\
\hline MWS-112 & - & - & - & 0.056 & $6.6 \times 10^{-7}$ & $1.1 \times 10^{-9}$ & 0.018 & $2.1 \times 10^{-7}$ & $3.4 \times 10^{-10}$ & - & - & - \\
\hline USGS-2 & - & - & - & - & - & - & - & - & - & - & - & - \\
\hline USGS-3 & - & - & - & 0.022 & $2.6 \times 10^{-7}$ & $4.1 \times 10^{-10}$ & 1.9 & $2.2 \times 10^{-6}$ & $3.6 \times 10^{-9}$ & - & - & - \\
\hline USGS-4 & - & - & - & 1.5 & $1.8 \times 10^{-5}$ & $2.8 \times 10^{-8}$ & 2.1 & $2.5 \times 10^{-5}$ & $3.9 \times 10^{-8}$ & - & - & - \\
\hline USGS-5 & - & - & - & - & - & - & - & - & - & - & - & - \\
\hline USGS-7 & - & - & - & - & - & - & - & - & - & - & - & - \\
\hline USGS-8 & - & - & - & - & - & - & - & - & - & - & - & - \\
\hline USGS-9 & - & - & - & 0.092 & $1.1 \times 10^{-6}$ & $1.7 \times 10^{-9}$ & 0.015 & $1.8 \times 10^{-7}$ & $2.8 \times 10^{-10}$ & - & - & - \\
\hline
\end{tabular}

a EPC values are maximum concentrations reported from the 1995 joint DOE/DA sampling rounds.

b Values in parentheses are inhalation intake estimates for TCE. The EPCs for TCE were derived from data obtained in 1996 and 1997 sampling activities at cheríical plant area wells.

c A hyphen (-) indicates the parameter was not detected. 
TABLE 3.9 Estimated Intakes of Uranium for the Hypothetical Future Resident

\begin{tabular}{|c|c|c|c|c|c|c|c|}
\hline \multirow[b]{3}{*}{ Well ID } & \multicolumn{3}{|c|}{ Uranium } & \multirow[b]{3}{*}{ Well ID } & \multicolumn{3}{|c|}{ Uranium } \\
\hline & \multirow{2}{*}{$\begin{array}{c}\mathrm{EPC}^{\mathrm{a}} \\
(\mathrm{pCi} / \mathrm{L})\end{array}$} & \multicolumn{2}{|c|}{ Intake (pCi) } & & \multirow{2}{*}{$\begin{array}{c}\mathrm{EPC}^{\mathrm{a}} \\
(\mathrm{pCi} / \mathrm{L})\end{array}$} & \multicolumn{2}{|c|}{ Intake $(\mathrm{pCi})$} \\
\hline & & Ingestion & Dermal & & & Ingestion & Dermal \\
\hline \multicolumn{4}{|l|}{ Deep Wells } & \multicolumn{2}{|c|}{ Unweathered (cont.) } & & \\
\hline MWD-05 & 0.58 & $1.2 \times 10^{4}$ & $1.9 \times 10^{1}$ & MW-3006 & 0.7 & $1.5 \times 10^{4}$ & $2.4 \times 10^{1}$ \\
\hline MWD-18 & 0.78 & $1.6 \times 10^{4}$ & $2.6 \times 10^{1}$ & MW-3024 & 3.1 & $6.4 \times 10^{4}$ & $1.0 \times 10^{2}$ \\
\hline MWGS-01 & - & - & - & MW-3026 & 4.2 & $9.0 \times 10^{4}$ & $1.4 \times 10^{2}$ \\
\hline MWGS-02 & - & - & - & MW-4004 & 2.1 & $4.5 \times 10^{4}$ & $7.2 \times 10^{1}$ \\
\hline MWS-18 & 1.5 & $3.2 \times 10^{4}$ & $5.2 \times 10^{1}$ & MW-4007 & 1.8 & $3.6 \times 10^{4}$ & $5.9 \times 10^{1}$ \\
\hline MWS-101 & 0.53 & $1.1 \times 10^{4}$ & $1.8 \times 10^{1}$ & MW-4008 & 0.83 & $1.7 \times 10^{4}$ & $2.8 \times 10^{1}$ \\
\hline MWS-102 & 2.6 & $5.4 \times 10^{4}$ & $8.7 \times 10^{1}$ & MW-4009 & 1.7 & $3.6 \times 10^{4}$ & $5.7 \times 10^{1}$ \\
\hline MWS-103 & 0.76 & $1.6 \times 10^{4}$ & $2.6 \times 10^{1}$ & MW-4011 & 3.1 & $6.6 \times 10^{4}$ & $1.0 \times 10^{2}$ \\
\hline \multirow[t]{2}{*}{ TIL-3 } & 0.11 & $2.2 \times 10^{3}$ & 3.6 & MW-4012 & 5.0 & $1.1 \times 10^{5}$ & $1.7 \times 10^{2}$ \\
\hline & & & & MW-4022 & 5.2 & $1.1 \times 10^{5}$ & $1.7 \times 10^{2}$ \\
\hline Overburden & & & & MWD-02 & 2.6 & $5.4 \times 10^{4}$ & $8.6 \times 10^{1}$ \\
\hline MW-2031 & - & - & - & MWD-06 & 0.58 & $1.2 \times 10^{4}$ & $1.9 \times 10^{1}$ \\
\hline MW-2032 & 4.2 & $8.8 \times 10^{4}$ & $1.4 \times 10^{2}$ & MWD-09 & 0.93 & $2.0 \times 10^{4}$ & $3.1 \times 10^{1}$ \\
\hline MW-2033 & 2.4 & $4.9 \times 10^{4}$ & $7.9 \times 10^{1}$ & MWD-23 & 5.0 & $1.1 \times 10^{5}$ & $1.7 \times 10^{2}$ \\
\hline MW-3001 & - & - & - & MWD-106 & - & - & - \\
\hline MW-3013 & - & - & - & MWS-05 & 0.99 & $2.1 \times 10^{4}$ & $3.3 \times 10^{1}$ \\
\hline MW-3018 & - & - & - & MWS-06 & 2.9 & $6.0 \times 10^{4}$ & $9.6 \times 10^{1}$ \\
\hline MW-3022 & - & - & - & MWS-105 & 1.3 & $3.6 \times 10^{-2}$ & 5.5 \\
\hline MW-3024 & - & - & - & MWS-106 & 1.6 & $4.4 \times 10^{-2}$ & $3.9 \times 10^{1}$ \\
\hline MWV-01 & 4.1 & $8.7 \times 10^{4}$ & $1.4 \times 10^{2}$ & MWS-109 & 1.0 & $2.7 \times 10^{-2}$ & $3.4 \times 10^{1}$ \\
\hline MWV-02 & 3 & $6.4 \times 10^{4}$ & $1.0 \times 10^{2}$ & TIL-4 & - & - & - \\
\hline MWV-09 & 0.72 & $1.5 \times 10^{4}$ & $2.4 \times 10^{1}$ & USGS-1 & 1.1 & $2.2 \times 10^{4}$ & $3.5 \times 10^{1}$ \\
\hline MWV-13 & 1.5 & $3.0 \times 10^{4}$ & $4.9 \times 10^{1}$ & USGS-6 & 1.8 & $4.9 \times 10^{-2}$ & $1.3 \times 10^{2}$ \\
\hline MWV-16 & 1.2 & $2.4 \times 10^{4}$ & $3.9 \times 10^{1}$ & & & & \\
\hline MWV-17 & 0.061 & $1.3 \times 10^{3}$ & 2.0 & Weathered & & & \\
\hline MWV-18 & - & - & - & MW-2001 & 0.65 & $1.4 \times 10^{4}$ & $2.2 \times 10^{1}$ \\
\hline MWV-22 & 0.88 & $1.9 \times 10^{4}$ & $3.0 \times 10^{1}$ & MW-2002 & 0.48 & $1.0 \times 10^{4}$ & $1.6 \times 10^{1}$ \\
\hline \multirow[t]{2}{*}{$M W V-24 R$} & 1.5 & $3.2 \times 10^{4}$ & $5.0 \times 10^{1}$ & MW-2003 & 1.1 & $2.2 \times 10^{4}$ & $3.6 \times 10^{1}$ \\
\hline & & & & MW-2004 & - & - & - \\
\hline Unweathered & & & & MW-2005 & 0.45 & $9.6 \times 10^{3}$ & $1.5 \times 10^{1}$ \\
\hline MW-2019 & 3.0 & $6.3 \times 10^{4}$ & $1.0 \times 10^{2}$ & MW-2006 & 0.48 & $1.0 \times 10^{4}$ & $1.6 \times 10^{1}$ \\
\hline MW-2021 & 0.87 & $1.8 \times 10^{4}$ & $2.9 \times 10^{1}$ & MW-2007 & 1.0 & $2.1 \times 10^{4}$ & $3.4 \times 10^{1}$ \\
\hline MW-2022 & 1.3 & $2.6 \times 10^{4}$ & $4.2 \times 10^{1}$ & MW-2008 & - & - & - \\
\hline MW-2023 & 2.5 & $5.3 \times 10^{4}$ & $8.5 \times 10^{1}$ & MW-2009 & - & - & - \\
\hline MW-2024 & 0.11 & $2.3 \times 10^{3}$ & 3.8 & MW-2010 & 1.2 & $2.6 \times 10^{4}$ & $4.1 \times 10^{1}$ \\
\hline MW-2025 & - & - & - & MW-2011 & 0.3 & $6.3 \times 10^{3}$ & 9.9 \\
\hline MW-2026 & 0.81 & $1.7 \times 10^{4}$ & $2.7 \times 10^{1}$ & MW-2012 & 0.33 & $6.9 \times 10^{3}$ & $1.1 \times 10^{1}$ \\
\hline MW-2027 & 0.81 & $1.7 \times 10^{4}$ & $2.7 \times 10^{1}$ & MW-2013 & 0.66 & $1.4 \times 10^{4}$ & $2.2 \times 10^{1}$ \\
\hline MW-2028 & 1.3 & $2.7 \times 10^{4}$ & $4.3 \times 10^{1}$ & MW-2014 & 0.49 & $1.0 \times 10^{4}$ & $1.6 \times 10^{1}$ \\
\hline MW-2029 & - & - & - & MW-2015 & 1.9 & $4.0 \times 10^{4}$ & $6.5 \times 10^{1}$ \\
\hline $\mathrm{MW}-3002$ & - & - & - & MW-2016 & - & - & - \\
\hline
\end{tabular}


TABLE 3.9 (Cont.)

\begin{tabular}{|c|c|c|c|c|c|c|c|}
\hline \multirow[b]{3}{*}{ Well ID } & \multicolumn{3}{|c|}{ Uranium } & \multirow[b]{3}{*}{ Well ID } & \multicolumn{3}{|c|}{ Uranium } \\
\hline & \multirow{2}{*}{$\begin{array}{c}\mathrm{EPC}^{\mathrm{a}} \\
(\mathrm{pCi} / \mathrm{L})\end{array}$} & \multicolumn{2}{|c|}{ Intake (pCi) } & & \multirow{2}{*}{$\begin{array}{c}\mathrm{EPC}^{\mathrm{a}} \\
(\mathrm{pCi} / \mathrm{L})\end{array}$} & \multicolumn{2}{|c|}{ Intake $(\mathrm{pC} \mathrm{i})$} \\
\hline & & Ingestion & Dermal & & & Ingestion & Dermal \\
\hline \multicolumn{4}{|c|}{ Weathered (cont.) } & \multicolumn{2}{|c|}{ Weathered (cont.) } & & \\
\hline MW-2017 & 12 & $2.5 \times 10^{5}$ & $4.0 \times 10^{2}$ & MW-4023 & 1.6 & $3.3 \times 10^{4}$ & $5.2 \times 10^{1}$ \\
\hline MW-2018 & 1.6 & $3.3 \times 10^{4}$ & $5.2 \times 10^{1}$ & MW-4024 & 60 & $1.3 \times 10^{6}$ & $2.0 \times 10^{3}$ \\
\hline MW-2020 & - & - & - & MW-4025 & 1.0 & $2.2 \times 10^{4}$ & $3.5 \times 10^{1}$ \\
\hline MW-2030 & 13 & $2.6 \times 10^{5}$ & $4.2 \times 10^{2}$ & MWD-15 & 0.49 & $1.0 \times 10^{4}$ & $1.7 \times 10^{1}$ \\
\hline MW-2034 & 3.0 & $6.3 \times 10^{4}$ & $1.0 \times 10^{2}$ & MWD-25 & 1.8 & $3.7 \times 10^{4}$ & $5.9 \times 10^{1}$ \\
\hline MW-2035 & 0.4 . & $8.4 \times 10^{3}$ & $1.3 \times 10^{1}$ & MWD-107 & 2 & $4.3 \times 10^{4}$ & $6.8 \times 10^{1}$ \\
\hline MW-2036 & 0.77 & $1.6 \times 10^{4}$ & $2.6 \times 10^{1}$ & MWD-112 & 0.77 & $1.6 \times 10^{4}$ & $2.6 \times 10^{1}$ \\
\hline MW-2037 & 1.2 & $2.6 \times 10^{5}$ & $4.2 \times 10^{1}$ & MWS-01 & 1.3 & $2.7 \times 10^{4}$ & $4.4 \times 10^{1}$ \\
\hline MW-2038 & 1.5 & $3.0 \times 10^{4}$ & $4.9 \times 10^{1}$ & MWS-02 & 2 & $4.2 \times 10^{4}$ & $6.8 \times 10^{1}$ \\
\hline MW-2039 & 3.1 & $6.6 \times 10^{4}$ & $1.0 \times 10^{2}$ & MWS-03 & 3.3 & $6.9 \times 10^{4}$ & $1.1 \times 10^{2}$ \\
\hline$M W-2040$ & 3.0 & $6.3 \times 10^{4}$ & $1.0 \times 10^{2}$ & MWS-04 & 10 & $2.1 \times 10^{5}$ & $3.4 \times 10^{2}$ \\
\hline$M W-2041$ & 3.4 & $6.9 \times 10^{4}$ & $1.1 \times 10^{2}$ & MWS-07 & 0.73 & $1.5 \times 10^{4}$ & $2.4 \times 10^{1}$ \\
\hline$M W-2042$ & 2.6 & $5.4 \times 10^{4}$ & $8.7 \times 10^{1}$ & MWS-08 & 1.1 & $2.4 \times 10^{4}$ & $3.8 \times 10^{1}$ \\
\hline$M W-2043$ & 1.8 & $3.9 \times 10^{4}$ & $6.0 \times 10^{1}$ & MWS-09 & 1.2 & $2.5 \times 10^{4}$ & $4.0 \times 10^{1}$ \\
\hline MW-2044 & 2.3 & $4.8 \times 10^{4}$ & $7.6 \times 10^{1}$ & MWS-10 & 0.13 & $2.8 \times 10^{3}$ & 4.5 \\
\hline MW-3003 & 19 & $3.9 \times 10^{5}$ & $6.3 \times 10^{2}$ & MWS-11 & 1.7 & $3.6 \times 10^{4}$ & $5.7 \times 10^{1}$ \\
\hline MW-3007 & - & - & - & MWS-12 & 1 & $2.1 \times 10^{4}$ & $3.4 \times 10^{1}$ \\
\hline MW-3008 & - & - & - & MWS-13 & 0.54 & $1.1 \times 10^{4}$ & $1.8 \times 10^{1}$ \\
\hline MW-3009 & - & - & - & MWS-14 & 2.7 & $5.6 \times 10^{4}$ & $9.0 \times 10^{1}$ \\
\hline MW-3010 & - & - & - & MWS-15 & 0.56 & $1.2 \times 10^{4}$ & $1.9 \times 10^{1}$ \\
\hline MW-3019 & 2.1 & $4.5 \times 10^{4}$ & $7.1 \times 10^{1}$ & MWS-16 & 0.66 & $1.4 \times 10^{4}$ & $2.2 \times 10^{1}$ \\
\hline MW-3023 & 13 & $2.7 \times 10^{5}$ & $4.3 \times 10^{2}$ & MWS-17 & 1.2 & $2.5 \times 10^{4}$ & $3.9 \times 10^{1}$ \\
\hline MW-3025 & 2.8 & $5.8 \times 10^{4}$ & $9.3 \times 10^{1}$ & MWS-19 & 1.3 & $2.7 \times 10^{4}$ & $4.3 \times 10^{1}$ \\
\hline MW-3027 & 1.3 & $2.6 \times 10^{4}$ & $4.2 \times 10^{1}$ & MWS-20 & 0.69 & $1.4 \times 10^{4}$ & $2.3 \times 10^{1}$ \\
\hline$M W-4001$ & 0.41 & $8.6 \times 10^{3}$ & $1.4 \times 10^{1}$ & MWS-21 & 3 & $6.3 \times 10^{4}$ & $1.0 \times 10^{2}$ \\
\hline$M W-4002$ & 0.6 & $1.3 \times 10^{4}$ & $2.0 \times 10^{1}$ & MWS-22 & 1.2 & $2.5 \times 10^{4}$ & $4.0 \times 10^{1}$ \\
\hline MW-4003 & 1.1 & $2.4 \times 10^{4}$ & $3.8 \times 10^{1}$ & MWS-24 & - & - & - \\
\hline$M W-4005$ & 1.6 & $3.3 \times 10^{4}$ & $5.3 \times 10^{1}$ & MWS-25 & 1.6 & $3.3 \times 10^{4}$ & $5.3 \times 10^{1}$ \\
\hline$M W-4006$ & 0.26 & $5.5 \times 10^{3}$ & 8.8 & MWS-26 & 4 & $8.3 \times 10^{4}$ & $1.3 \times 10^{2}$ \\
\hline$M W-4010$ & 3.1 & $6.4 \times 10^{4}$ & $1.0 \times 10^{2}$ & MWS-104 & 1.3 & $2.7 \times 10^{4}$ & $4.4 \times 10^{1}$ \\
\hline MW-4013 & 1.2 & $2.5 \times 10^{4}$ & $4.0 \times 10^{1}$ & MWS-107 & 1.8 & $3.8 \times 10^{4}$ & $6.1 \times 10^{1}$ \\
\hline MW-4014 & 0.22 & $4.7 \times 10^{3}$ & 7.5 & MWS-110 & 0.63 & $1.3 \times 10^{4}$ & $2.1 \times 10^{1}$ \\
\hline MW-4015 & 0.32 & $6.7 \times 10^{3}$ & $1.1 \times 10^{1}$ & MWS-112 & 2.7 & $5.7 \times 10^{4}$ & $9.1 \times 10^{1}$ \\
\hline MW-4016 & 3.2 & $6.7 \times 10^{4}$ & $1.1 \times 10^{2}$ & USGS-2 & 0.001 & $2.1 \times 10^{1}$ & $3.4 \times 10^{-2}$ \\
\hline MW-4017 & - & - & - & USGS-3 & 1.4 & $3.0 \times 10^{4}$ & $4.8 \times 10^{1}$ \\
\hline MW-4018 & 0.64 & $1.4 \times 10^{4}$ & $2.1 \times 10^{1}$ & USGS-4 & 0.54 & $1.1 \times 10^{4}$ & $1.8 \times 10^{1}$ \\
\hline$M W-4019$ & 1.7 & $3.6 \times 10^{4}$ & $5.7 \times 10^{1}$ & USGS-5 & 4.9 & $1.0 \times 10^{5}$ & $1.6 \times 10^{2}$ \\
\hline MW-4020 & 9.7 & $2.0 \times 10^{5}$ & $3.3 \times 10^{2}$ & USGS-8 & 0.62 & $1.3 \times 10^{4}$ & $2.1 \times 10^{1}$ \\
\hline MW-4021 & 3.1 & $6.6 \times 10^{4}$ & $1.0 \times 10^{2}$ & USGS-9 & 0.35 & $7.2 \times 10^{3}$ & $1.2 \times 10^{1}$ \\
\hline
\end{tabular}

a EPC values are the maximum concentrations reported for uranium from each well from the 1995 joint DOE/DA sampling rounds. 
TABLE 3.10 Exposure Factors for the American Robin ${ }^{a}$

\begin{tabular}{lcccc}
\hline \multicolumn{1}{c}{ Exposure Factor } & Mean & $\begin{array}{c}\text { Range or } \\
95 \% \text { UCL }\end{array}$ & $\begin{array}{c}\text { Geographic } \\
\text { Location }\end{array}$ & Source \\
\hline Body weight $(\mathrm{g})$ & 77 & $63-100$ & Pennsylvania & Clench and Leberman (1978) \\
Water ingestion rate (g/g-d) & 0.14 & - & - & Estimated $^{\mathrm{b}}$ \\
Home range (ha) & 0.81 & - & Ontario & Weatherhead and McRae (1990) \\
\hline
\end{tabular}

a A hyphen (-) indicates that the information was not applicable or not available.

b Estimated using the following allometric equations (EPA 1993):

Water Ingestion Rate $(\mathrm{L} / \mathrm{d})=0.059 \mathrm{~W}^{0.67}$, where $\mathrm{W}$ equals weight $(0.077 \mathrm{~kg})$; and

Normalized Water Ingestion Rate $(\mathrm{g} / \mathrm{g}-\mathrm{d})=($ Water Ingestion $[\mathrm{g} / \mathrm{d}]) \div \mathrm{W}(\mathrm{g})$.

TABLE 3.11 Exposure Factors for the White-Tailed Deer ${ }^{a}$

\begin{tabular}{lcccc}
\hline \multicolumn{1}{c}{ Exposure Factor } & Mean & $\begin{array}{c}\text { Range or } \\
95 \% \text { UCL }\end{array}$ & $\begin{array}{c}\text { Geographic } \\
\text { Location }\end{array}$ & Source \\
\hline Body weight (g) & 90,000 & - & Missouri & Schwartz and Schwartz (1981) \\
Water ingestion rate (g/g-d) & 0.06 & - & - & Estimated $^{\mathrm{b}}$ \\
Home range (ha) & 160 & 260 & Missouri & Schwartz and Schwartz (1981) \\
\hline
\end{tabular}

a A hyphen (-) indicates that the information was not applicable or not available.

b Estimated using the following allometric equations (EPA 1993):

Water Ingestion Rate $(\mathrm{L} / \mathrm{d})=0.099 \mathrm{~W}^{0.90}$, where W equals weight $(90.0 \mathrm{~kg})$; and

Normalized Water Ingestion Rate $(\mathrm{g} / \mathrm{g}-\mathrm{d})=($ Water Ingestion $[\mathrm{g} / \mathrm{d}]) \div \mathrm{W}(\mathrm{g})$. 
TABLE 3.12 Results of Acute and Chronic Toxicity Testing of Surface Water from Burgermeister Spring ${ }^{a}$

Toxicity Test Results at Sampling Location ${ }^{\mathrm{b}}$

Organism/Toxicity Test SP-6301-1 SP-6301-2 SP-6301-3 SP-6301-4

Daphnia, 96-hour acute survival

Hyalella, 96-hour acute survival

Pimephales, 96-hour acute survival

$$
37.5 \% \text { survival }
$$

$37.5 \%$ survival

Xenopus, 96-hour acute survival

Daphnia, 7-day chronic survival

Hyalella, 7-day chronic survival

Pimephales, 7-day chronic survival and growth

Xenopus, 7-day chronic survival and growth

a A minus (-) indicates no significant media toxicity $(p>0.05)$; a plus $(+)$ indicates significant media toxicity $(\mathrm{p} \leq 0.05)$.

b Sampling locations SP-6301-1 through SP-6301-4 are from Burgermeister Spring.

c $\mathrm{NC}=$ chronic toxicity testing not conducted because media toxicity at this sampling location was indicated by the results of the corresponding acute toxicity test. 
TABLE 3.13 Results of Acute and Chronic Toxicity Testing of Sediment from Burgermeister Spring ${ }^{a}$

Toxicity Test Results at Sampling Location ${ }^{\mathrm{b}}$

Organism/Toxicity Test

SP-6301-1

SP-6301-2

SP-6301-3

SP-6301-4

Daphnia, 96-hour acute survival

Hyalella, 96-hour acute survival

Pimephales, 96-hour acute survival

Xenopus, 96-hour acute survival

Daphnia, 7-day chronic survival

Hyalella, 7-day chronic survival

Pimephales, 7-day chronic survival and growth

\begin{tabular}{cccc}
- & - & - & - \\
- & - & - & - \\
- & + & - & - \\
- & $75 \%$ survival & & - \\
- & - & - & - \\
+ & - & - & - \\
$82 \%$ survival & - & - & + \\
- & - & - & $50 \%$ survival \\
- & + & - & - \\
\hline
\end{tabular}

Xenopus, 7-day chronic survival and growth

$73 \%$ survival

a A minus (-) indicates no significant media toxicity $(\mathrm{p}>0.05)$; a plus $(+)$ indicates significant media toxicity $(\mathrm{p} \leq 0.05)$.

b Sampling locations SP-6301-1 through SP-6301-4 are from Burgermeister Spring. 
TABLE 3.14 Estimated Applied Daily Dose from the Drinking Water Pathway for the American Robin and White-Tailed Deer

\begin{tabular}{|c|c|c|c|}
\hline \multirow[b]{2}{*}{ Contaminant } & \multirow{2}{*}{$\begin{array}{c}\text { EPC } \\
(\mu \mathrm{g} / \mathrm{L})\end{array}$} & \multicolumn{2}{|c|}{ Applied Daily Dose $^{\mathrm{a}}(\mathrm{mg} / \mathrm{kg}-\mathrm{d})$} \\
\hline & & American Robin & White-Tailed Deer \\
\hline \multicolumn{4}{|l|}{ Metals } \\
\hline Aluminum & 2,800 & 0.38 & $<0.01$ \\
\hline Antimony & 95 & 0.01 & $<0.01$ \\
\hline Arsenic & 290 & $<0.04$ & $<0.01$ \\
\hline Barium & 3,200 & 0.44 & $<0.01$ \\
\hline Cadmium & 25 & $<0.01$ & $<0.01$ \\
\hline Chromium & 30 & $<0.01$ & $<0.01$ \\
\hline Copper & 30 & $<0.01$ & $<0.01$ \\
\hline Iron & 400,000 & 55 & 0.01 \\
\hline Lead & 60 & $<0.01$ & $<0.01$ \\
\hline Lithium & 52 & $<0.01$ & $<0.01$ \\
\hline Manganese & 20,000 & 2.8 & 0.02 \\
\hline Mercury & 6,100 & 0.84 & $<0.01$ \\
\hline Molybdenum & 38 & $<0.01$ & $<0.01$ \\
\hline Nickel & 44 & $<0.01$ & $<0.01$ \\
\hline Selenium & 6 & $<0.01$ & $<0.01$ \\
\hline Silver & 240 & $<0.03$ & $<0.01$ \\
\hline Strontium & 190 & 0.03 & $<0.01$ \\
\hline Thallium & 6 & $<0.01$ & $<0.01$ \\
\hline Uranium, total & 540 & 0.09 & $<0.01$ \\
\hline \multicolumn{4}{|l|}{ Inorganic anion } \\
\hline Nitrate-N & 10,000 & 1.4 & 0.001 \\
\hline \multicolumn{4}{|c|}{ Nitroaromatic compounds } \\
\hline $1,3,5$-TNB & 15 & $<0.01$ & $<0.01$ \\
\hline 1,3-DNB & 1 & $<0.01$ & $<0.01$ \\
\hline $2,4,6-\mathrm{TNT}$ & 280 & 0.04 & $<0.01$ \\
\hline $2,4-\mathrm{DNT}$ & 11 & $<0.01$ & $<0.01$ \\
\hline 2,6-DNT & 18 & $<0.01$ & $<0.01$ \\
\hline 2-Amino-4,6-DNT & 19 & $<0.01$ & $<0.01$ \\
\hline 4-Amino-2,6-DNT & 24 & $<0.01$ & $<0.01$ \\
\hline Nitrotoluene & 0.08 & $<0.01$ & $<0.01$ \\
\hline Nitrobenzene & 1 & $<0.01$ & $<0.01$ \\
\hline
\end{tabular}

a Dose estimates were calculated using Burgermeister Spring as the exposure point area and using the maximum contaminant concentrations reported from all springs in the chemical plant area and the ordnance works area as the EPCs. 


\section{TOXICITY ASSESSMENT}

The toxicities of the radioactive and chemical COPCs and COECs identified for the GWOUs are summarized in Sections 4.1 and 4.2. The methods used to evaluate toxicity are discussed in Section 4.3.

\subsection{RADIATION TOXICITY}

\subsubsection{Human Health}

Uranium was identified as the only radioactive COPC for the GWOUs. Natural uranium consists of three isotopes: uranium-234, uranium-235, and uranium-238. These isotopes have very low radioactivity per gram of material (i.e., specific activity) due to their long half-lives. Two hazards are associated with uranium compounds: kidney damage caused by the chemical toxicity and cell damage caused by the ionizing radiation that results from radioactive decay. Alpha, beta, and gamma radiation are released during the radioactive decay of uranium. For internal exposures (e.g., by ingestion or inhalation), alpha and beta radiation are the primary hazards. Within the body, alpha particles result in greater cell damage than beta or gamma radiation because their energy is completely absorbed by the tissue. Beta particles deposit less energy to tissue and therefore induce much less damage than alpha particles. Gamma radiation is primarily an external hazard because it can easily penetrate tissue and reach internal organs.

\subsubsection{Ecological Health}

Identifying the effects of radionuclides on organisms in the natural environment is complicated because (1) various sources of ionizing radiation are possible; (2) exposure can be internal, external, or both; (3) each radionuclide has unique physical and chemical properties; (4) ecological receptors have different mobilities and varied habitats; and (5) current levels of radionuclides in most areas are too low to detect effects on population and community, even in such areas as weapons testing sites (Whicker and Schultz 1982a-b). Effects due to acute or chronic exposure include mortality, physiological and pathological changes, and developmental and reproductive effects (National Council on Radiation Protection and Measurements [NCRP] 1991; International Atomic Energy Agency [IAEA] 1992; Rose 1992).

Ecological receptors may be affected by both acute and chronic exposure to ionizing radiation. For acute exposure, aquatic invertebrates tend to be more resistant than aquatic vertebrates. The most sensitive periods in the life cycle of aquatic organisms are the early developmental stages; radiation sensitivity generally decreases with increasing development (NCRP 1991). Reproductive and early developmental stages of aquatic organisms are most sensitive to chronic irradiation. 
Deleterious effects of chronic irradiation have not been observed in natural populations at dose rates $\leq 1 \mathrm{rad} / \mathrm{d}(\mathrm{NCRP} 1991)$.

Similar sensitivity and effects have been identified for terrestrial wildlife (IAEA 1992). Terrestrial invertebrates are much less sensitive than terrestrial vertebrates, the invertebrates requiring about 100 times the dose needed for vertebrates to induce mortality. Among vertebrate species, lethal acute doses and sensitivity to chronic radiation vary widely among different taxa; birds, mammals, and a few tree species are among the most sensitive. Acute doses of $<10$ rad are considered unlikely to produce persistent, measurable deleterious changes in populations or communities of terrestrial plants or animals (IAEA 1992). Chronic dose rates of $<0.1 \mathrm{rad} / \mathrm{d}$ and $<1 \mathrm{rad} / \mathrm{d}$ do not appear likely to cause observable changes in terrestrial animal populations, and chronic dose rates of $<1 \mathrm{rad} / \mathrm{d}$ are not likely to cause observable changes in plant populations. As with aquatic biota, reproductive and early developmental stages of terrestrial biota are most sensitive to irradiation.

\subsection{CHEMICAL TOXICITY}

\subsubsection{Human Health}

The chemical COPCs in groundwater include lithium, molybdenum, uranium, chloride, nitrates, sulfates, nitroaromatic compounds, TCE, and 1,2-DCE. The chemical COPCs in spring water include antimony, iron, lithium, manganese, mercury, molybdenum, silver, uranium, chloride, nitrate, sulfate, and nitroaromatic compounds.

Antimony is typically present in soil as sulfide and oxide compounds. Industrially, antimony is used in many alloys. It has been administered orally to humans and animals as both an emetic and an antiparasitic agent. Toxic effects that have been observed in humans are associated mainly with occupational exposures.

Iron is an essential nutrient present at varying levels in the human diet; the recommended daily allowance (RDA) ranges from 6 to $30 \mathrm{mg} / \mathrm{d}$ for infants and pregnant women, respectively; the RDA for adults is $15 \mathrm{mg} / \mathrm{d}$ (National Research Council 1989). Approximately 2,000 cases of iron poisoning occur in the United States annually, primarily among young children who ingest adult iron supplements; the lethal dose of iron is about $200 \mathrm{mg} / \mathrm{kg}$, at least 200 times the RDA level.

Lithium is present in the daily human diet at a level of about $2 \mathrm{mg}$ (Venugopal and Luckey 1978). It is safely used as a psychiatric drug at concentrations of about $1 \mathrm{~g} / \mathrm{d}$, and lithium carbonate is used clinically to treat depression. Toxic effects that have been observed subsequent to treatment include effects on the neuromuscular and cardiovascular systems, irritation of the gastrointestinal tract, and kidney damage. 
Manganese is an essential dietary nutrient for humans and is present in many foods. Studies of humans and experimental animals suggest that oral exposure to elevated levels of manganese can result in decreased fertility and in effects on the cardiovascular and central nervous systems.

Inorganic and organic forms of mercury have been found to be toxic in humans and experimental animals. In general, the organic forms are more toxic that the inorganic forms. Human studies indicate that the kidney and central nervous system are the main sites affected by mercury; however, the degree to which these systems are affected depends on the chemical form of mercury and the route of exposure.

Molybdenum is a trace element present in the daily human diet at levels of about $0.2 \mathrm{mg} / \mathrm{d}$. It is a constituent of several enzymes, but nutritional requirements are low and molybdenum deficiencies are extremely rare. Elevated dietary levels (i.e., in excess of about $10 \mathrm{mg} / \mathrm{d}$ ) are associated with a condition characterized by swelling, inflammation, and pain in the joints (EPA 1997).

Natural uranium is radioactive, but the primary health effect associated with exposure to uranium is kidney damage caused by chemical toxicity. The oral reference dose (RfD) derived for soluble salts of uranium is based on decreased body weight and moderate kidney damage induced in rabbits fed with uranyl nitrate hexahydrate for 30 days (Maynard and Hodge 1949).

Inorganic anions such as nitrates and chloride occur naturally in the environment in soils and in plant and animal food products. Nitrates are commonly found in the environment as a result of urban sewage treatment, nitrogenous wastes, and nitrogen-based fertilizers. The health hazards associated with nitrates result primarily from the bacterial conversion of ingested nitrates to nitrites, which can result in methemoglobinemia (reduction in the oxygen-carrying capacity of blood), especially in infants. Chloride is the main inorganic anion found in the blood and extracellular fluids and is essential in maintaining fluid and electrolyte balance. Added salt in foods is the primary source of ingested chloride, contributing about $6 \mathrm{~g} / \mathrm{d}$ (National Research Council 1989). Additional chloride from water is typically insignificant, averaging about $40 \mathrm{mg} / \mathrm{d}$. The toxicity of salts containing the chloride ion depends primarily on the characteristics of the cation (e.g., sodium in table salt, which has been associated with high blood pressure). Sulfates are commonly found in the environment and are widely used for industrial purposes. Sulfates exhibit low toxicity in humans but have been shown to have laxative effects at water concentrations of $630 \mathrm{mg} / \mathrm{L}$ or greater (Chien et al. 1968).

Health hazards associated with nitroaromatic compounds include methemoglobinemia and toxic effects on the liver, kidneys, and nervous system. Studies in humans indicate that nitroaromatic compounds are absorbed following inhalation and ingestion and that these compounds are capable of penetrating the skin. Human exposure to TCE primarily affects the central nervous system. Effects include headaches, vertigo, fatigue, and central nervous system depression. 


\subsubsection{Ecological Health}

The COECs include metals and nitroaromatic compounds. Metals have been reported to cause a variety of lethal and sublethal effects in aquatic and terrestrial biota. The toxicity of these contaminants depends on physical and chemical factors in the environment, such as $\mathrm{pH}$ and the presence of complexing agents, as well as on the specific taxon being exposed. In vegetation, reported adverse effects of metal exposure include reduced chlorophyll concentrations, reduced growth and biomass production, and reduced seed production and germination. In aquatic biota, metal exposure has been shown to affect reproduction, ion exchange across gill surfaces, behavior, and survival of all life stages. In terrestrial biota, metal exposure can result in developmental abnormalities; renal and central nervous system damage; altered blood chemistry; altered metabolic processes; and behavioral changes affecting foraging, susceptibility to predators, and reproduction.

Relatively little information is available regarding the effects of nitroaromatic compounds on natural populations of plants, fish, and wildlife. Laboratory studies have shown that exposure to nitroaromatic compounds causes a variety of responses in aquatic and terrestrial biota. Effects of exposure on fish and aquatic invertebrates include increased adult mortality, reduced egg production and survival, decreased survival of early life stages, reduced body weights and lengths, and increased physical deformities. Adverse effects on aquatic plants may include depressed growth and cellular deformities.

Effects of nitroaromatic compounds on terrestrial wildlife may include reduced body weights, changes in blood chemistry and cellular composition, changes in metabolic pathways and processes, renal and liver malfunction, and organ necroses and lesions. Reported effects to terrestrial vegetation include reduced leaf and root growth, reduced plant height, and leaf and root necroses.

\subsection{METHODS FOR EVALUATING TOXICITY TO HUMAN HEALTH}

\subsubsection{Radiation Toxicity}

The assessment of radiological human health risks in this BRA was limited to carcinogenic effects. This approach is consistent with EPA guidance, which notes that cancer risk is generally the limiting effect for radionuclides and suggests that radiation carcinogenesis be used as the sole basis for assessing radiation-related human health risks (EPA 1989b). Carcinogenic risks were calculated for the radionuclides of concern in a manner similar to existing methods for chemical carcinogens by using an age-averaged lifetime excess cancer incidence per unit intake. To support this evaluation, the EPA has developed cancer incidence factors per unit intake that are synonymous with the slope factors developed for chemical carcinogens.

The following radionuclide slope factors were used in this assessment: uranium-234, $4.4 \times 10^{-11} / \mathrm{pCi}$; uranium-235, $4.5 \times 10^{-11} / \mathrm{pCi}$; and uranium-238+D, $6.2 \times 10^{-11} / \mathrm{pCi}($ EPA $1995 \mathrm{c}$ ). 
The " $+\mathrm{D}$ " designation indicates that the risks from associated short-lived decay products (i.e., with radioactive half-lives less than or equal to 6 months) are also included. Only ingestion slope factors have been used because inhalation and external radiation are not pathways of concern for the receptors being assessed. The activity-weighted average of these slope factors for isotopic conditions present in site groundwater $\left(5.3 \times 10^{-11} / \mathrm{pCi}\right)$ was used in conjunction with the total concentration of uranium (in $\mathrm{pCi} / \mathrm{L}$ ) to estimate the radiological risk.

\subsubsection{Chemical Toxicity}

The EPA has derived toxicity values for most of the chemical contaminants of human health concern and assigned RfDs to measure the noncarcinogenic effects of chemicals. The chronic $\mathrm{RfD}$ is defined as "an estimate of a daily exposure level for the human population, including sensitive subpopulations, that is likely to be without an appreciable risk of deleterious effects during a lifetime" (EPA 1989b). To derive an RfD value (expressed in $\mathrm{mg} / \mathrm{kg}-\mathrm{d}$ ), EPA reviews all toxicity studies available for a given substance and a given route of exposure, determines a no-observedadverse-effect level (NOAEL) or a lowest-observed-adverse-effect level (LOAEL) from the study most relevant to humans (the critical study), and applies uncertainty factors to these values. The RfD can be compared with estimated exposure levels to evaluate the potential for deleterious effects. Current available $\mathrm{RfD}$ values are specific to either the inhalation or ingestion route of exposure because the toxic mechanism and dose required for toxicity to occur can differ for these routes of exposure. For this BRA, only ingestion RfDs have been used because ingestion has been determined to be the pathway of concern for the receptors being assessed. Oral RfDs are available for the following COPCs for the GWOUs: antimony, lithium, manganese, mercury, molybdenum, silver, uranium, nitrate, 1,3,5-TNB, 1,3-DNB, 2,4,6-TNT, 2,4-DNT, 2,6-DNT, 2-amino-4,6-DNT, 4-amino-2,6-DNT, nitrobenzene, and o-, m-, and p-nitrotoluene.

The toxic effects of short-term exposures to the COPCs are not generally evaluated because the risks estimated for chronic low-level exposures are greater than the short-term toxicity risks. However, nitrate toxicity in infants is an exception; in infants, toxicity may occur after a short period of ingestion. The EPA Office of Drinking Water has derived 1-day and 10-day health advisory levels to assess concentrations of concern for short-term exposures; the 1-day and 10-day health advisory levels for nitrate are both $10 \mathrm{mg} / \mathrm{L}$ (EPA 1997). The short-term toxicity of nitrate was assessed by using infant exposure parameters as well as adult exposure parameters to calculate hazard indices (see Chapter 5). The use of infant exposure parameters resulted in a calculated hazard index of 1 for a well with a nitrate concentration of $10 \mathrm{mg} / \mathrm{L}$.

Carcinogenic risks from exposure to known and potential carcinogens are evaluated separately from noncarcinogenic risks because, hypothetically, any exposure to a carcinogen increases the risk of cancer by a finite amount. Therefore, the risk from exposure to a carcinogen at a given level can be derived, but an exposure level at which no carcinogenic effect is likely to occur (as for noncarcinogenic end points) cannot be defined. The EPA has defined two toxicity values for evaluating the potential carcinogenic effects of a given substance: the weight-of-evidence classification and the slope factor. For substances that have weight-of-evidence classifications of A (human 
carcinogen), B1 or B2 (probable human carcinogens), and sometimes C (possible human carcinogens), the EPA has calculated slope factors on the basis of data from dose-response studies. The slope factor is defined as a "plausible upper-bound estimate of the probability of a response (i.e., cancer) per unit intake of a chemical over a lifetime" (EPA 1989b). Generally, slope factors are derived by extrapolation from experimental high-dose to low-dose ranges, and they are not valid for evaluating high dose levels. Also, carcinogenic risks that have been calculated from slope factors are applicable to exposures that occur over a lifetime. When exposure durations are less than a lifetime, they must be converted to equivalent lifetime values. The following COPCs at the GWOU have verified slope factors: TCE, 2,4,6-TNT, 2,4-DNT, and 2,6-DNT. All RfD values and slope factors are summarized in Tables 4.1 and 4.2 
TABLE 4.1 Toxicity Values of COPCs for Ingestion of Groundwater and Surface Water: Potential Systemic Effects

\begin{tabular}{|c|c|c|c|c|c|c|}
\hline \multirow[b]{2}{*}{ Parameter } & \multirow{2}{*}{$\begin{array}{c}\text { Chronic RfD } \\
(\mathrm{mg} / \mathrm{kg}-\mathrm{d})\end{array}$} & \multirow{2}{*}{$\begin{array}{c}\text { Level of } \\
\text { Confidence }\end{array}$} & \multirow[b]{2}{*}{ Critical Effect } & \multicolumn{2}{|c|}{$\mathrm{RfD}$} & \multirow{2}{*}{$\begin{array}{l}\text { Uncertainty } \\
\text { Factor }^{\mathrm{b}} \text { (UF) }\end{array}$} \\
\hline & & & & Basis & Source $^{a}$ & \\
\hline \multicolumn{7}{|l|}{ Metals } \\
\hline Antimony & 0.0004 & Low & Reduced lifespan; altered blood chemistry & Oral, rat & IRIS & $\mathrm{UF}=1,000$ \\
\hline Lithium & $0.02^{\mathrm{c}}$ & Medium & Impaired renal function & Oral & $-c$ & $\mathrm{UF}=100$ \\
\hline Manganese & 0.14 & Medium & Effects on central nervous system & Diet, human & IRIS & $\mathrm{UF}=1$ \\
\hline $\begin{array}{l}\text { Mercury (as mercuric } \\
\text { chloride) }\end{array}$ & 0.0003 & High & Autoimmune effects & Diet, rat & IRIS & $\mathrm{UF}=1,000$ \\
\hline Molybdenum & 0.005 & $N A^{d}$ & Changes in biochemical indexes & Oral, human & IRIS & $\mathrm{UF}=1$ \\
\hline Silver & 0.005 & Low & Argyria (skin discoloration) & Intravenous & IRIS & $\mathrm{UF}=3$ \\
\hline Uranium & 0.003 & Medium & Weight loss; moderate kidney activity & Oral, rabbit & IRIS & $\mathrm{UF}=1,000$ \\
\hline \multicolumn{7}{|l|}{ Inorganic anion } \\
\hline Nitrate-N & 1.6 & High & Methemoglobinemia & Oral, human & IRIS & $\mathrm{UF}=1$ \\
\hline \multicolumn{7}{|l|}{ Nitroaromatic compounds } \\
\hline $1,3,5-\mathrm{TNB}$ & 0.00005 & Low & Increased splenic weight & Oral, rat & IRIS & $\mathrm{UF}=10,000$ \\
\hline 1,3-DNB & 0.0001 & Low & Increased splenic weight & Oral, rat & IRIS & $\mathrm{UF}=3,000$ \\
\hline $2,4,6-\mathrm{TNT}$ & 0.0005 & Medium & Liver effects & Oral, dog & IRIS & $\mathrm{UF}=1,000$ \\
\hline 2,4-DNT & 0.002 & High & Neurotoxicity; bilary tract hyperplasia; Heinz bodies & Oral, dog & IRIS & $\mathrm{UF}=100$ \\
\hline 2,6-DNT & 0.001 & NA & Neurotoxicity; bilary tract hyperplasia; Heinz bodies & Oral & HEAST & $\mathrm{UF}=3,000$ \\
\hline 2-Amino-4,6-DNT & $0.00006^{\mathrm{c}}$ & Low & Neurotoxicity; bilary tract hyperplasia; Heinz bodies & Oral & $-\mathbf{c}$ & $\mathrm{UF}=10,000$ \\
\hline 4-Amino-2,6-DNT & $0.00006^{\mathrm{C}}$ & Low & Neurotoxicity; bilary tract hyperplasia; Heinz bodies & Oral & $-^{c}$ & $\mathrm{UF}=10,000$ \\
\hline Nitrotoluene & 0.001 & NA & Splenic lesions & Oral, rat & HEAST & $\mathrm{UF}=10,000$ \\
\hline Nitrobenzene & 0.0005 & Low & Hematological, adrenal, renal, and hepatic lesions & $\begin{array}{c}\text { Inhalation, rat } \\
\text { and mouse }\end{array}$ & IRIS & $\mathrm{UF}=10,000$ \\
\hline
\end{tabular}

a Source: Integrated Risk Information System (EPA 1997), except as indicated.

b The NOAEL or LOAEL dose from the critical study can be obtained by multiplication of the chronic RfD by the uncertainty factor.

c Provisional value provided by the EPA's Superfund Technical Support Center (Baysinger-Daniel 1996).

d $\mathrm{NA}=$ not available. 
TABLE 4.2 Toxicity Values of COPCs for Ingestion of Groundwater and Spring Water: Potential Carcinogenic Effects

\begin{tabular}{|c|c|c|c|c|c|}
\hline \multirow[b]{2}{*}{ Parameter } & \multirow{2}{*}{$\begin{array}{l}\text { Slope Factor } \\
{\left[(\mathrm{mg} / \mathrm{kg}-\mathrm{d})^{-1}\right]}\end{array}$} & \multirow{2}{*}{$\begin{array}{c}\text { Weight of Evidence } \\
\text { Classification }\end{array}$} & \multirow[b]{2}{*}{ Type of Cancer } & \multicolumn{2}{|c|}{ Slope Factor } \\
\hline & & & & Basis & Source $^{\mathrm{a}}$ \\
\hline $2,4,6-\mathrm{TNT}$ & 0.03 & C: possible human carcinogen & $\begin{array}{l}\text { Urinary bladder; transitional cell papilloma; } \\
\text { transitional squamous carcinoma }\end{array}$ & Diet, rat & IRIS \\
\hline 2,4-DNT & 0.68 & B2: probable human carcinogen & $\begin{array}{l}\text { Liver, mammary gland; adenocarcinomas/ } \\
\text { carcinomas }\end{array}$ & Water, rat & IRIS \\
\hline 2,6-DNT & 0.68 & B2: probable human carcinogen & $\begin{array}{l}\text { Liver, mammary gland; adenocarcinomas/ } \\
\text { carcinomas }\end{array}$ & Water, rat & IRIS \\
\hline TCE & $0.011^{\mathrm{b}}$ & B2: probable human carcinogen & Liver & $N A^{c}$ & $-\mathbf{b}$ \\
\hline
\end{tabular}

a Source: Integrated Risk Information System (EPA 1997), except as indicated.

b TCE slope factor for the inhalation pathway is 0.006 (EPA 1996b).

c NA = not available. 


\section{HUMAN HEALTH RISK CHARACTERIZATION}

Radiological and chemical health risks to humans were characterized for exposure to contamination in groundwater and spring water at the chemical plant area and ordnance works area. Potential carcinogenic risks for both radiological and chemical exposures were measured in terms of the increased probability that an individual would develop cancer over a lifetime. The EPA has indicated that for known or suspected carcinogens, acceptable exposure levels for members of the general public at sites on the National Priorities List (NPL) are generally concentration levels that represent an excess upper-bound lifetime cancer risk to an individual of between $1 \times 10^{-6}$ and $1 \times 10^{-4}$ (EPA 1989b). This range is referred to as the "acceptable risk range" in this BRA and is used as a point of reference for discussing the results of the carcinogenic risk assessment for the GWOU.

Potential health effects from exposure to chemical contaminants were also assessed for effects other than cancer. The quantitative measures of noncarcinogenic health effects are the hazard quotient and hazard index (see Section 5.1.2.2). The EPA has defined a hazard index of greater than 1 as the level of concern for noncarcinogenic health effects.

\subsection{RISK CHARACTERIZATION METHODOLOGY}

\subsubsection{Radiological Risks}

Exposures to low levels of ionizing radiation can result in cancer, serious genetic effects, and other detrimental health effects. The induction of cancer is the predominant radiological effect associated with uranium, the only radioactive COPC identified in groundwater and spring water at the chemical plant and ordnance works areas. The radiological health risks presented in this BRA are limited to carcinogenic effects. This approach is consistent with EPA guidance, which notes that, in general, the risk of cancer is limiting and may be used as the sole basis for assessing the radiationrelated human health risks for a site contaminated with radionuclides (EPA 1989b).

For this assessment, slope factors were used to estimate the potential risk from exposure to radionuclides. Intakes were estimated (in units of pCi) for the ingestion pathway (see Chapter 3). Radiological risks were then calculated by multiplying the intakes by the appropriate slope factor (see Section 4.3.1). 


\subsubsection{Chemical Risks and Hazard Quotients}

\subsubsection{Carcinogenic Risks}

The risk to human health from exposure to chemical carcinogens is expressed as the probability of a cancer occurring over a lifetime. To calculate the excess cancer risk, the daily intake averaged over a lifetime is multiplied by a chemical-specific slope factor. The EPA has derived slope factors for a number of carcinogens. These slope factors represent the incremental lifetime cancer risk per milligram of carcinogen per kilogram of body weight, assuming that the exposure occurs over a lifetime of 70 years. The estimated daily intakes (averaged over a lifetime) resulting from exposure to the chemical carcinogens in the groundwater and spring water are presented in Section 3.3.1; available slope factors are identified in Section 4.3.2.

\subsubsection{Hazard Quotients and Hazard Indices}

A hazard quotient provides a measure of the potential for adverse health effects other than cancer. For an individual contaminant, the daily intake averaged over the exposure period is divided by the RfD to derive the hazard quotient. The RfD is the average daily dose that can be incurred without an appreciable risk of deleterious health effects during a lifetime. The EPA has derived chronic RfDs for exposure periods of more than 7 years; only chronic RfDs were considered in this assessment.

For an individual contaminant, a hazard quotient of 1 or greater is considered to indicate a potential for adverse health effects. The individual hazard quotients for each contaminant are summed to determine the hazard index.

\subsection{HUMAN HEALTH RISK ESTIMATES}

\subsubsection{Spring Water}

The risk to a recreational visitor from exposure to contaminants at the springs was estimated consistent with current and projected future land uses. Calculations were performed for each of the 15 springs to determine both radiological and chemical carcinogenic risks. Hazard indices were determined for each spring to assess potential noncarcinogenic adverse health effects from the chemical contaminants. 


\subsubsection{Chemical Risks and Hazard Indices}

The hazard indices estimated for the recreational visitor at the 15 springs are very low; the estimated range is $<0.001$ to 0.2 , indicating that contaminant concentrations at the springs are not likely to result in noncarcinogenic health effects to the recreational visitor. The highest hazard index of 0.2 was reported for spring SP-5201, located south of the groundwater divide. The second highest hazard index was also reported for a spring south of the groundwater divide, SP-5303, with a hazard index of 0.1. The primary contributors are 2-amino-4,6-DNT, 4-amino-2,6-DNT, and 2,4,6-TNT. Estimated hazard quotients for the COPCs are presented in Table $5.1 ;^{1}$ the distribution of estimated hazard indices for the recreational visitor is illustrated in Figure 5.1.

The chemical carcinogenic risk estimates for the springs evaluated range from $2 \times 10^{-10}$ to $3 \times 10^{-7}$ (Table 5.2); all of these estimates fall below the acceptable risk range. The highest risk estimates were reported for SP-5201 and SP-5303, which are located south of the groundwater divide. These results indicate that chemical carcinogenicity is not a factor at the springs. The distribution of these risk estimates is illustrated in Figure 5.2.

The projected chemical carcinogenic and noncarcinogenic toxicity for the future recreational visitor is expected to be no higher than the estimated current risks due to attenuation of contaminant concentrations expected as a result of source removals. The hazard indices and carcinogenic risks for the Army reservist scenario at the springs can be expected to be no greater than those estimated for the recreational visitor because exposure parameters for the Army reservist scenario are projected to be similar to those assumed for the recreational visitor.

\subsubsection{Radiological Risks}

Potential radiological risks from exposure to contaminants at the springs for the current and future recreational visitor range from $4 \times 10^{-9}(\mathrm{SP}-5602)$ to $2 \times 10^{-6}$ (SP-5303), as shown in Table 5.3. These values are low, and all but two of the estimates are below the lower end of the acceptable risk range. Overall, these results indicate that there is minimal risk to the recreational visitor, including the Army reservist, from potential exposure to radioactive contaminants at the springs. These results are depicted in Figure 5.2.

\subsubsection{Groundwater}

A hazard index was calculated for each of the 155 wells in the monitoring networks at the chemical plant area and ordnance works area to determine potential noncarcinogenic or systemic effects for a hypothetical future resident exposed to water from these wells. Estimated radiological and chemical carcinogenic risks to this hypothetical receptor were also calculated. Hazard quotients

1 All tables in this chapter have been placed at the of the text (Section 5.5). 


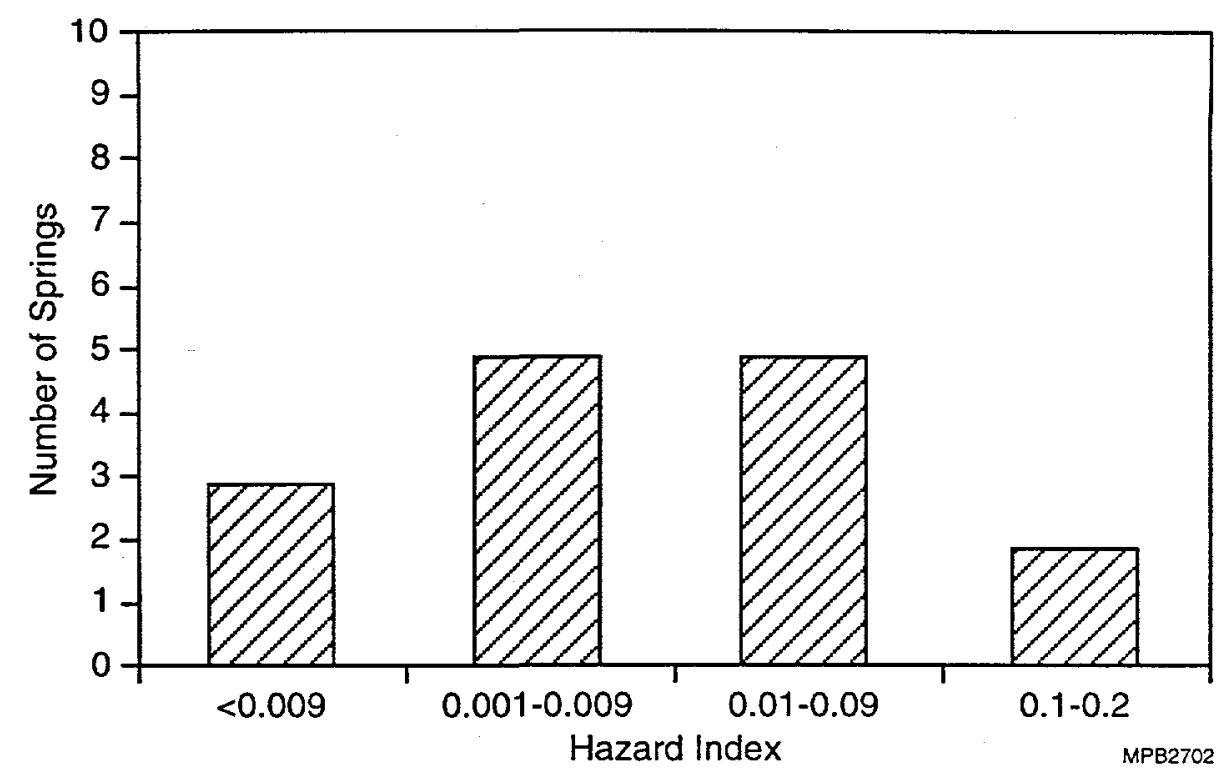

FIGURE 5.1 Distribution of Estimated Hazard Indices for the Current and Future Recreational Visitor at the Chemical Plant Area and Ordnance Works Area

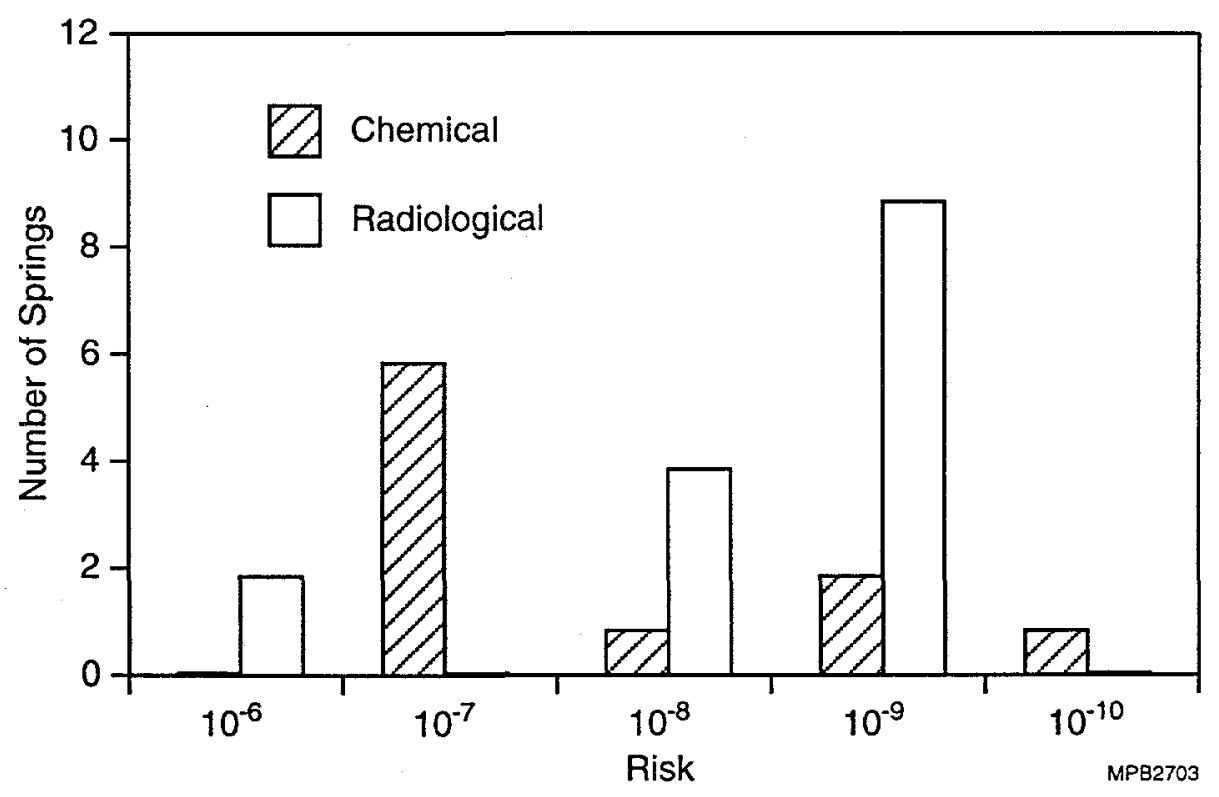

FIGURE 5.2 Distribution of Estimated Chemical and Radiological Carcinogenic Risks for the Current and Future Recreational Visitor at the Chemical Plant Area and Ordnance Works Area 
and carcinogenic risks from dermal contact with groundwater while showering were not estimated because intakes from this pathway (see Section 3.3.2) were only a fraction of the ingestion intakes.

\subsubsection{Chemical Risks and Hazard Indices}

The estimated hazard indices for the hypothetical future adult resident range from $<0.01$ to 40 , as shown in Table 5.4. Overall, the estimated hazard indices were 1 or greater for 43 of the 155 wells evaluated (Figure 5.3 illustrates these estimates). Further analysis of these results indicates that 27 of the 43 estimates greater than 1 were due primarily to nitroaromatic compounds and 15 to nitrates. The hazard index in well MW-4024 was estimated to be 1 ; uranium contributed to about 0.84 of the total hazard index of 1 . Overall, contributions to the hazard index from lithium and uranium were minimal in comparison to nitrates and nitroaromatic compounds. Hazard indices also were generally higher for wells completed in the weathered unit.

The toxic effect of nitrate (i.e., methemoglobinemia, or low blood oxygen levels) is primarily of concern for infants (EPA 1997). Therefore, a separate hazard quotient was calculated for infants ingesting groundwater, assuming an ingestion rate of $0.64 \mathrm{~L} / \mathrm{d}$ and a body weight of $4 \mathrm{~kg}$ (compared with the adult intake of $2 \mathrm{~L} / \mathrm{d}$ and body weight of $70 \mathrm{~kg}$ ). The results show an increased hazard quotient for nitrate in each well by a factor of approximately 5.6. By calculating the hazard index assuming infant exposure parameters, an additional 5 wells have a hazard index exceeding 1.

The chemical carcinogenic risk estimates, excluding contributions from TCE, range from $1 \times 10^{-7}$ to $2 \times 10^{-4}$, as shown in Table 5.5. Of the 155 wells evaluated, estimates for only four wells were at or slightly greater than $1 \times 10^{-4}$. The highest risk of $2 \times 10^{-4}$ was estimated for wells MWV-09 and MWS-12. The primary contributors to these estimated risks were 2,4-DNT and 2,6-DNT. Carcinogenic risks tend to be higher for weathered wells, which is consistent with the estimated hazard indices. These risk results indicate that any future residential well completed in the deeper aquifer (i.e., deeper than the unweathered and deep wells presented in this report) would not result in unacceptable risk. In fact, three of the four wells that exceeded the acceptable risk range are weathered wells; the fourth, MWV-09, is an overburden well. Figure 5.4 shows the distribution of the risk estimates.

During 1996 and 1997 sampling activities, TCE was detected in nine wells at or in the vicinity of the chemical plant area, primarily in the area around the raffinate pits. The total risks at these wells, including the TCE contributions, were estimated to be $2 \times 10^{-5}$ for MW-3024, $4 \times 10^{-4}$ for MW-2037, $1 \times 10^{-3}$ for MW-2038, $1 \times 10^{-5}$ for MW-3025, $1 \times 10^{-6}$ for MW-4001, $2 \times 10^{-7}$ for MW-2032, $3 \times 10^{-4}$ for MWS-21, $9 \times 10^{-7}$ for MW-3027, and $6 \times 10^{-7}$ for MW-2013. Of these, estimates for only three wells were greater than the acceptable risk range of $1 \times 10^{-4}$. 


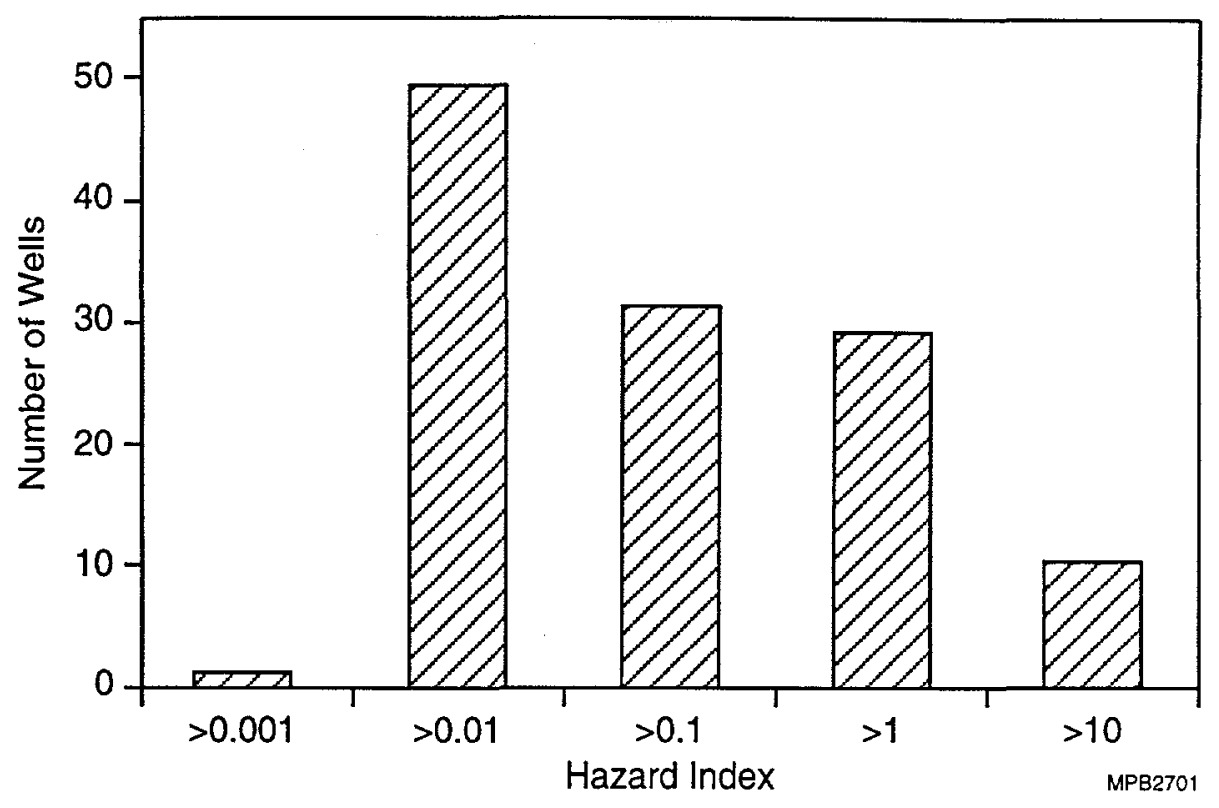

FIGURE 5.3 Distribution of Estimated Hazard Indices for the Hypothetical Future Resident at the Chemical Plant Area and Ordnance Works Area

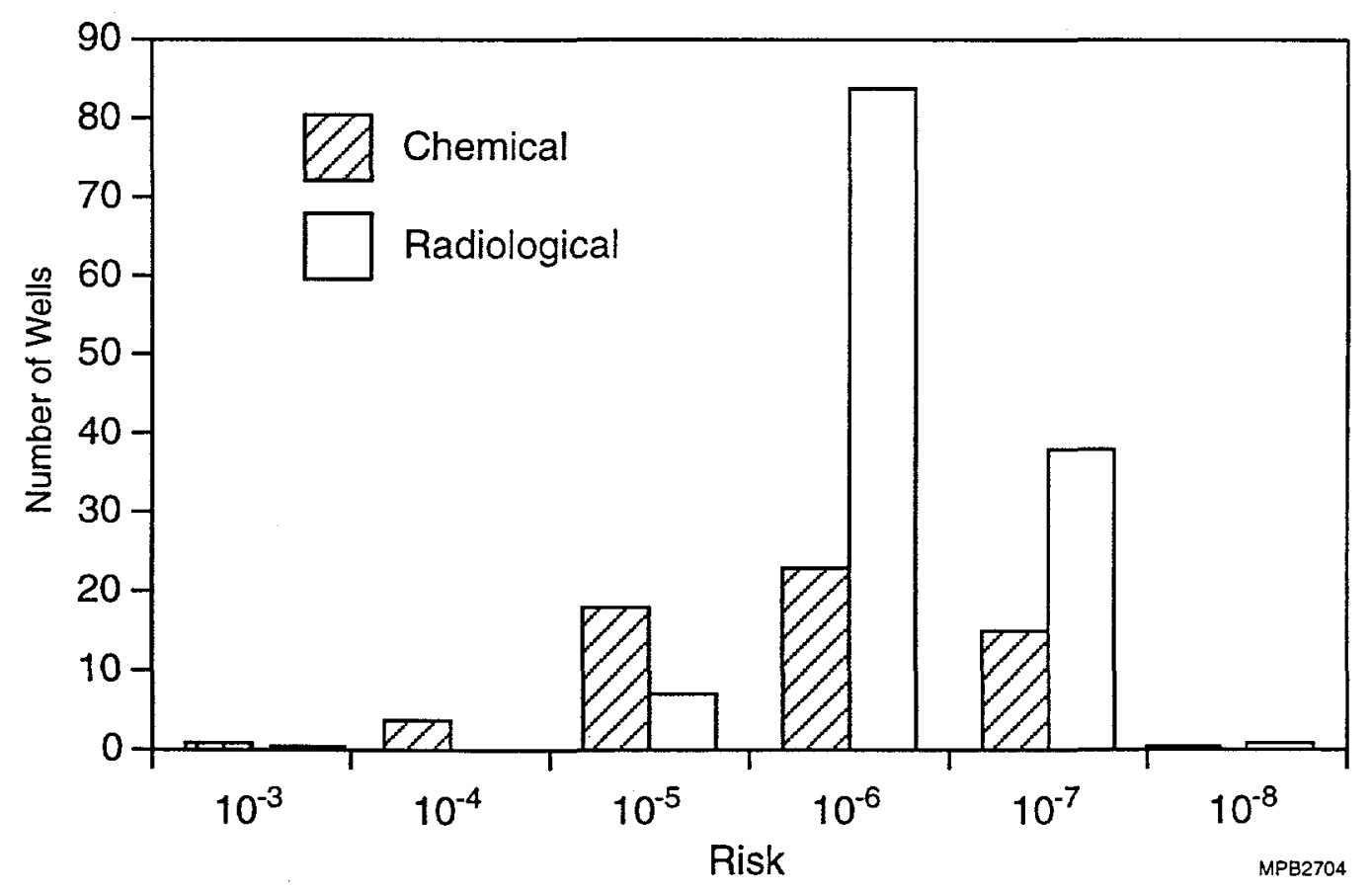

FIGURE 5.4 Distribution of Estimated Chemical and Radiological Carcinogenic Risks for the Hypothetical Future Resident at the Chemical Plant Area and Ordnance Works Area 


\subsubsection{Radiological Risks}

The estimated radiological risks for a future resident ingesting water from wells in the chemical plant area and ordnance works area are at the low end of or are lower than the acceptable risk range recommended by the EPA (Table 5.6). The estimated radiological risk ranges from $7 \times 10^{-8}$ to $7 \times 10^{-5}$. The radiological risk estimates for all wells evaluated are depicted in Figure 5.4. Similarly to chemical risk estimates, the higher radiological risk estimates are for weathered wells, mostly located around the raffinate pits and other source areas at the chemical plant area.

\subsection{UNCERTAINTIES RELATED TO RISK ESTIMATES}

The evaluation of risks to human health presented in this BRA was by necessity based on a number of assumptions. In addition, many uncertainties are inherent in the risk assessment process. The rationale for major assumptions used in this assessment and associated uncertainties are discussed in Sections 5.3.1 through 5.3.4.

\subsubsection{Contaminants of Potential Concern}

The identification of COPCs for the human health evaluation relied on use of monitoring data for both areas and applying a selection process recommended by the EPA. The monitoring wells at the chemical plant area and ordnance works area are considered to adequately characterize groundwater flow and monitor changes in water quality at these areas. Data from these wells therefore establish the nature and extent of contamination and are expected to provide an adequate database for identifying COPCs with sufficient certainty.

\subsubsection{Exposure Assessment}

The amount and type of data available and the ability to address fate and transport impacts over time affect the determination of representative EPCs. The quantity of data has been determined to be sufficient for this risk assessment. Exposure point concentrations used to project current and hypothetical future risks were based on current (about 1995) maximum concentrations for both the groundwater and spring water analyses. This approach is considered to be conservative. With source removals currently occurring at both areas, it is expected that the COPC concentrations will decrease with time.

The uranium concentrations detected from the in-situ sampling of groundwater at the Southeast Drainage were higher than the uranium concentrations detected at the current monitoring network wells. The higher uranium concentrations may be an artifact of the field sampling methodology. Initial data from a recently installed monitoring well in this area have indicated uranium to be present at levels below the detection limit (DOE and DA 1997). 
Some uncertainty is associated with the assumptions used to identify scenarios and intake parameters. Site-specific factors were used to identify the potential receptors (e.g., recreational visitor to the area springs) and to select the scenario assumptions, such as extent of exposure (i.e., exposure time, frequency, and duration). These assumptions incorporate information on current land use and reasonable projections of future land use that consider the time frame of the assessment. The uncertainty in the selected scenarios is low because federal and state ownership of surrounding land is expected to continue into the foreseeable future. The surrounding wildlife areas are the most heavily used in the state, and future plans include further expansion of the recreational use of the area. Therefore, a recreational visitor scenario is considered appropriate for both current and future conditions.

Considerable information is available for the ingestion pathway with respect to reasonable assumptions for intake parameters (e.g., ingestion rate), so related uncertainty is expected to be low. To estimate the reasonable maximum exposures for the identified receptors, best professional judgment was used in defining the variables that determine the extent of exposure. Intake parameters used in the exposure assessment were derived from data in the literature, including values provided by the EPA (1995b). Default parameters were supplemented on a chemical-specific basis, as appropriate (i.e., by including nitrate exposure estimates for infants). Because the exposure parameters generally represent the 95 th percentile of the distributions, combining them results in a point intake estimate that represents an even higher percentile for the overall exposure. Thus, in some cases, the "reasonable" representative exposure may be somewhat overestimated.

The approach used to calculate the dermal pathway tends to be conservative in that critical contaminant-specific factors such as absorption fractions and permeability coefficients are not available. Conservative (high) default values for permeability coefficients were used in the calculations.

\subsubsection{Toxicity Assessment}

Standard RfDs and slope factors established by the EPA were used to estimate potential noncarcinogenic and carcinogenic health effects from exposure to chemical contaminants at the GWOUs of the chemical plant area and ordnance works area.

No RfD values or slope factors are available for chloride or iron. However, because these substances are naturally occurring and are present at some concentration in food and water, the National Research Council (1989) has published RDA values, which may be used as indicators of safe levels to be ingested in groundwater. The RDAs for a $10-\mathrm{kg}$ infant are 300 and $10 \mathrm{mg} / \mathrm{d}$, for chloride and iron, respectively. Assuming an ingestion rate of $1 \mathrm{~L} / \mathrm{d}$ for a $10-\mathrm{kg}$ infant, water concentrations of $300 \mathrm{mg} / \mathrm{L}$ chloride and $10 \mathrm{mg} / \mathrm{L}$ iron could correspond to intake levels exceeding the RDA levels. None of the monitoring wells have EPC levels exceeding $300 \mathrm{mg} / \mathrm{L}$ for chloride, and none of the springs exceeded levels of $10 \mathrm{mg} / \mathrm{L}$ for iron. This suggests that there is no health 
concern associated with chloride in groundwater or iron in spring water at the chemical plant area and the ordnance works area.

Sulfate also does not have an RfD value or slope factor available. Sulfate is generally of low toxicity; the only adverse health impact associated with it is a laxative effect starting at water levels of about $700 \mathrm{mg} / \mathrm{L}$ (National Research Council 1977); this water would be unlikely to be ingested because the taste threshold is about $300 \mathrm{mg} / \mathrm{L}$. Only one well has a sulfate EPC exceeding $700 \mathrm{mg} / \mathrm{L}$ (MW-2017); water from this well would not be ingested because of its objectionable taste and smell.

\subsubsection{Risk Characterization}

The radiological and chemical risk assessments have been presented separately because the methodologies for estimating carcinogenic risks from exposures to radionuclides and chemicals differ considerably. However, the total carcinogenic risk to an individual is that resulting from exposure to both the radiological and chemical risks, assuming that carcinogenic effects are neither antagonistic nor synergistic.

\subsection{SUMMARY}

Carcinogenic (radiological and chemical) risk and systemic toxicity are not indicated for the recreational visitor potentially exposed to contaminants in spring water. The recreational visitor was considered to be the most likely receptor, accounting for current and expected future land uses for both the chemical plant area and ordnance works area. Potential incremental carcinogenic risk and systemic toxicity to the Army reservists are not indicated.

Calculations performed to evaluate potential risks for the hypothetical future resident using groundwater indicate that concentrations of nitroaromatic compounds in a few wells contribute to slightly greater than the upper end of the acceptable risk range $\left(1 \times 10^{-4}\right)$ recommended by the EPA. Several wells in the vicinity of known source areas at the chemical plant area (e.g., raffinate pits) contain high concentrations of nitrates that contribute to hazard indices greater than 1 ; sludge at the pits has been determined to contain high concentrations of nitrates. Several wells at various locations in both the chemical plant area and ordnance works area also contain levels of nitroaromatic compounds that potentially contribute to both carcinogenic and noncarcinogenic toxicity for an individual exposed to these waters. Radiological carcinogenic risk estimates for uranium are within the acceptable risk range; the higher estimates are attributable to chemical plant area wells that monitor known source areas such as the raffinate pits, Ash Pond, and Frog Pond. However, uranium risk estimates from the in-situ samples obtained at the Southeast Drainage indicate that radiological risk could be somewhat higher. In addition, potential risk for the future resident exposed to contaminants at the springs would be minimal relative to the groundwater pathways. 


\subsection{CUMULATIVE EXPOSURE THROUGH MULTIPLE MEDIA}

The current recreational visitor is not expected to incur additional risk from site soil because site areas with contaminated soil are not readily accessible by the general public. For the future recreational visitor scenario, the hazard indices and carcinogenic risks estimated in this analysis should be representative of the potential total exposure incurred by this receptor, accounting for potential exposure to site soil in addition to area springs. Remediation goals for soil cleanup are set to achieve human health protection at levels of $1 \times 10^{-6}$ and lower.

Similarly, for the hypothetical future resident scenario, the carcinogenic risks and hazard indices incurred from exposure to residual site soil contamination would be minimal $\left(1 \times 10^{-6}\right.$ or lower and 1 or lower, respectively). Therefore, the potential total exposure incurred by this receptor would be no greater than the value estimated in this analysis (see Section 5.2.2), except possibly for a few locations with radium-226 soil contamination at the chemical plant area. An incremental concentration of radium-226 of $0.075 \mathrm{pCi} / \mathrm{g}$ corresponds to a risk of $1 \times 10^{-4}$. The background radium-226 concentration is $1.2 \mathrm{pCi} / \mathrm{g}$. 
TABLE 5.1 Estimated Hazard Quotients for COPCs for the Current and Future

Recreational Visitor

\begin{tabular}{|c|c|c|c|c|c|c|c|c|}
\hline \multirow{3}{*}{$\begin{array}{c}\text { Spring } \\
\text { ID }\end{array}$} & \multicolumn{8}{|c|}{ Estimated Hazard Quotient } \\
\hline & \multicolumn{2}{|c|}{ Antimony } & \multicolumn{2}{|c|}{ Lithium } & \multicolumn{2}{|c|}{ Manganese } & \multicolumn{2}{|c|}{ Mercury } \\
\hline & Ingestion & Dermal & Ingestion & Dermal & Ingestion & Dermal & Ingestion & Dermal \\
\hline 5101 & 0.001 & 0.00002 & 0.00002 & 0.0000004 & 0.00006 & 0.000001 & $--^{a}$ & - \\
\hline 5201 & 0.0008 & 0.00002 & - & - & 0.000006 & 0.0000001 & - & - \\
\hline 5303 & 0.001 & 0.00002 & 0.00007 & 0.000001 & 0.00003 & 0.0000007 & 0.0005 & 0.00001 \\
\hline 5402 & 0.0007 & 0.00002 & - & - & 0.00001 & 0.0000002 & - & - \\
\hline 5501 & 0.001 & 0.00002 & 0.00002 & 0.0000004 & 0.00003 & 0.0000007 & - & - \\
\hline 5504 & 0.0006 & 0.00001 & - & - & 0.000007 & 0.0000001 & - & - \\
\hline 5601 & 0.001 & 0.00003 & - & - & 0.0003 & 0.000005 & - & - \\
\hline 5602 & 0.003 & 0.00007 & - & - & 0.0002 & 0.000005 & - & - \\
\hline 5605 & 0.001 & 0.00002 & - & - & 0.00004 & 0.0000008 & - & - \\
\hline 5612 & 0.005 & 0.0001 & - & - & 0.00001 & 0.0000002 & - & - \\
\hline 6301 & 0.0008 & 0.00002 & 0.0001 & 0.000003 & 0.00002 & 0.0000004 & - & - \\
\hline 6303 & 0.002 & 0.00005 & 0.00003 & 0.0000006 & 0.00006 & 0.000001 & - & - \\
\hline 6306 & 0.0009 & 0.00002 & - & - & 0.01 & 0.0002 & - & - \\
\hline 6501 & 0.006 & 0.00001 & - & - & 0.00003 & 0.0000007 & - & - \\
\hline 6601 & 0.002 & 0.00004 & - & - & 0.00005 & 0.000001 & - & - \\
\hline
\end{tabular}


TABLE 5.1 (Cont.)

\begin{tabular}{|c|c|c|c|c|c|c|c|c|}
\hline \multirow{3}{*}{$\begin{array}{c}\text { Spring } \\
\text { ID }\end{array}$} & \multicolumn{8}{|c|}{ Estimated Hazard Quoticnt } \\
\hline & \multicolumn{2}{|c|}{ Molybdenum } & \multicolumn{2}{|c|}{ Silver } & \multicolumn{2}{|c|}{ Uranium, Total } & \multicolumn{2}{|c|}{ Nitrate } \\
\hline & Ingestion & Dermal & Ingestion & Dermal & Ingestion & Dermal & Ingestion & Dermal \\
\hline 5101 & - & - & - & - & 0.00003 & 0.0000006 & 0.0002 & 0.000005 \\
\hline 5201 & - & - & - & - & 0.00007 & 0.000001 & 0.00002 & 0.0000004 \\
\hline 5303 & 0.0004 & 0.000009 & - & - & 0.01 & 0.0002 & 0.0002 & 0.000004 \\
\hline 5402 & - & - & - & - & 0.00007 & 0.000002 & 0.00004 & 0.0000009 \\
\hline 5501 & - & - & - & - & 0.00006 & 0.000001 & 0.00004 & 0.0000008 \\
\hline 5504 & - & - & 0.00005 & 0.000001 & 0.00004 & 0.0000008 & - & - \\
\hline 5601 & - & - & - & - & 0.00004 & 0.0000007 & - & - \\
\hline 5602 & - & - & - & - & 0.00003 & 0.0000005 & 0.00005 & 0.000001 \\
\hline 5605 & - & - & - & - & 0.00005 & 0.000001 & 0.00001 & 0.0000003 \\
\hline 5612 & - & - & - & - & 0.00004 & 0.0000009 & - & - \\
\hline 6301 & - & - & 0.00006 & 0.000001 & 0.005 & 0.0001 & 0.002 & 0.00004 \\
\hline 6303 & - & - & - & - & 0.0001 & 0.000002 & 0.001 & 0.00003 \\
\hline 6306 & 0.0002 & 0.000003 & - & - & 0.00005 & 0.000001 & - & - \\
\hline 6501 & - & - & 0.001 & 0.00002 & 0.0002 & 0.000004 & 0.00004 & 0.0000009 \\
\hline 6601 & - & - & - & - & 0.00003 & 0.0000006 & 0.00007 & 0.000002 \\
\hline
\end{tabular}


TABLE 5.1 (Cont.)

\begin{tabular}{|c|c|c|c|c|c|c|c|c|c|c|}
\hline \multirow{3}{*}{$\begin{array}{l}\text { Spring } \\
\text { ID }\end{array}$} & \multicolumn{10}{|c|}{ Estimated Hazard Quotient } \\
\hline & \multicolumn{2}{|c|}{$1,3,5$-TNB } & \multicolumn{2}{|c|}{ 1,3-DNB } & \multicolumn{2}{|c|}{ 2,4,6-TNT } & \multicolumn{2}{|c|}{ 2,4-DNT } & \multicolumn{2}{|c|}{ 2,6-DNT } \\
\hline & Ingestion & Dermal & Ingestion & Dermal & Ingestion & Dermal & Ingestion & Dermal & Ingestion & Dermal \\
\hline 5101 & - & - & - & - & - & - & - & - & - & - \\
\hline 5201 & 0.02 & 0.0004 & - & - & 0.03 & 0.0007 & 0.000006 & 0.0000001 & 0.0003 & 0.000006 \\
\hline 5303 & 0.001 & 0.00003 & - & - & 0.04 & 0.0008 & 0.00002 & 0.0000004 & 0.00006 & 0.000001 \\
\hline 5402 & - & - & - & - & - & - & - & - & - & - \\
\hline 5501 & - & - & - & - & - & - & 0.000004 & 0.00000008 & 0.00001 & 0.0000003 \\
\hline 5504 & - & - & - & - & - & - & - & - & - & - \\
\hline 5601 & - & - & - & - & - & - & 0.000003 & 0.00000007 & 0.000008 & 0.0000002 \\
\hline 5602 & 0.0008 & 0.00002 & - & - & 0.0003 & 0.000007 & 0.00001 & 0.0000002 & 0.0003 & 0.000007 \\
\hline 5605 & 0.0003 & 0.000006 & - & - & 0.002 & 0.00003 & 0.00001 & 0.0000003 & 0.00004 & 0.0000009 \\
\hline 5612 & - & - & - & - & 0.00002 & 0.0000005 & - & - & - & - \\
\hline 6301 & 0.00007 & 0.000002 & - & - & 0.00008 & 0.000002 & 0.000005 & 0.0000001 & 0.00004 & 0.0000007 \\
\hline 6303 & 0.0003 & 0.000006 & - & - & 0.0005 & 0.00001 & 0.00001 & 0.0000003 & 0.00006 & 0.000001 \\
\hline 6306 & - & - & - & - & - & - & - & - & - & - \\
\hline 6501 & - & - & - & - & - & - & - & - & - & - \\
\hline 6601 & - & - & - & - & 0.000006 & 0.0000001 & - & - & 0.000008 & 0.0000002 \\
\hline
\end{tabular}




\section{TABLE 5.1 (Cont.)}

\begin{tabular}{|c|c|c|c|c|c|c|c|c|c|}
\hline \multirow{4}{*}{$\begin{array}{l}\text { Spring } \\
\text { ID }\end{array}$} & \multicolumn{9}{|c|}{ Estimated Hazard Quotient } \\
\hline & \multirow{2}{*}{\multicolumn{2}{|c|}{ 2-Amino-4,6-DNT }} & \multirow{2}{*}{\multicolumn{2}{|c|}{ 4-Amino-2,6-DNT }} & \multirow{2}{*}{\multicolumn{2}{|c|}{ Nitrobenzene }} & \multicolumn{3}{|c|}{ Total } \\
\hline & & & & & & & & & \\
\hline & Ingestion & Dermal & Ingestion & Dermal & Ingestion & Dermal & Ingestion & Dermal & + Dermal \\
\hline 5101 & - & - & - & - & - & - & 0.001 & 0.00003 & 0.001 \\
\hline 5201 & 0.050 & 0.0010 & 0.05 & 0.001 & - & - & 0.2 & 0.003 & 0.2 \\
\hline 5303 & 0.02 & 0.00050 & 0.04 & 0.0008 & - & - & 0.1 & 0.002 & 0.1 \\
\hline 5402 & - & - & - & - & - & - & 0.0009 & 0.00002 & 0.0009 \\
\hline 5501 & 0.0006 & 0.00001 & 0.001 & 0.000020 & - & - & 0.003 & 0.00006 & 0.003 \\
\hline 5504 & - & - & - & - & - & - & 0.0007 & 0.00001 & 0.0007 \\
\hline 5601 & 0.0006 & 0.00001 & 0.001 & 0.00002 & - & - & 0.003 & 0.00007 & 0.003 \\
\hline 5602 & 0.002 & 0.00005 & 0.003 & 0.00007 & - & - & 0.01 & 0.0002 & 0.01 \\
\hline 5605 & 0.004 & 0.00009 & 0.007 & 0.0002 & - & - & 0.01 & 0.0003 & 0.01 \\
\hline 5612 & 0.001 & 0.00003 & 0.002 & 0.00003 & - & - & 0.008 & 0.0002 & 0.008 \\
\hline 6301 & 0.002 & 0.00004 & 0.004 & 0.00007 & - & - & 0.01 & 0.0003 & 0.01 \\
\hline 6303 & 0.003 & 0.00007 & 0.006 & 0.0001 & - & - & 0.01 & 0.0003 & 0.01 \\
\hline 6306 & - & - & - & - & - & - & 0.01 & 0.0002 & 0.01 \\
\hline 6501 & 0.00005 & 0.000001 & 0.00009 & 0.000002 & - & - & 0.002 & 0.00004 & 0.002 \\
\hline 6601 & 0.001 & 0.00002 & 0.002 & 0.00003 & - & - & 0.005 & 0.0001 & 0.005 \\
\hline
\end{tabular}

${ }^{a}$ A hyphen (-) indicates that the parameter was not detected. 
TABLE 5.2 Estimated Chemical Carcinogenic Risks for the Current and Future Recreational Visitor ${ }^{\mathrm{a}}$

\begin{tabular}{|c|c|c|c|c|c|c|c|c|c|}
\hline \multirow[b]{3}{*}{$\begin{array}{l}\text { Spring } \\
\text { ID }\end{array}$} & \multicolumn{9}{|c|}{ Estimated Risk } \\
\hline & \multicolumn{2}{|c|}{$2,4,6-\mathrm{TNT}$} & \multicolumn{2}{|c|}{ 2,4-DNT } & \multicolumn{2}{|c|}{ 2,6-DNT } & \multicolumn{3}{|c|}{ Total } \\
\hline & Ingestion & Dermal & Ingestion & Dermal & Ingestion & Dermal & Ingestion & Dermal & $\begin{array}{l}\text { Ingestion } \\
+ \text { Dermal }\end{array}$ \\
\hline 5201 & $2.2 \times 10^{-7}$ & $4.6 \times 10^{-9}$ & $3.5 \times 10^{-9}$ & $7.3 \times 10^{-11}$ & $8.2 \times 10^{-8}$ & $1.7 \times 10^{-9}$ & $3.1 \times 10^{-7}$ & $6.4 \times 10^{-9}$ & $3 \times 10^{-7}$ \\
\hline 5303 & $2.4 \times 10^{-7}$ & $5.1 \times 10^{-9}$ & $9.6 \times 10^{-9}$ & $2.0 \times 10^{-10}$ & $1.9 \times 10^{-8}$ & $3.9 \times 10^{-10}$ & $2.7 \times 10^{-7}$ & $5.7 \times 10^{-9}$ & $3 \times 10^{-7}$ \\
\hline 5501 & $-{ }^{b}$ & - & $2.1 \times 10^{-9}$ & $4.4 \times 10^{-11}$ & $3.4 \times 10^{-9}$ & $7.2 \times 10^{-11}$ & $5.5 \times 10^{-9}$ & $1.2 \times 10^{-10}$ & $6 \times 10^{-9}$ \\
\hline 5601 & - & - & $1.8 \times 10^{-9}$ & $3.8 \times 10^{-11}$ & $2.3 \times 10^{-9}$ & $4.9 \times 10^{-11}$ & $4.1 \times 10^{-9}$ & $8.7 \times 10^{-11}$ & $4 \times 10^{-9}$ \\
\hline 5602 & $2.0 \times 10^{-9}$ & $4.2 \times 10^{-11}$ & $5.9 \times 10^{-9}$ & $1.2 \times 10^{-10}$ & $9.1 \times 10^{-8}$ & $1.9 \times 10^{-9}$ & $9.9 \times 10^{-8}$ & $2.1 \times 10^{-9}$ & $1 \times 10^{-7}$ \\
\hline 5605 & $9.7 \times 10^{-9}$ & $2.0 \times 10^{-10}$ & $6.8 \times 10^{-9}$ & $1.4 \times 10^{-10}$ & $1.2 \times 10^{-8}$ & $2.6 \times 10^{-10}$ & $2.9 \times 10^{-8}$ & $6.0 \times 10^{-10}$ & $3 \times 10^{-8}$ \\
\hline 5612 & $1.5 \times 10^{-10}$ & $3.1 \times 10^{-12}$ & - & - & - & - & $1.5 \times 10^{-10}$ & $3.1 \times 10^{-12}$ & $2 \times 10^{-10}$ \\
\hline 6301 & $5.0 \times 10^{-10}$ & $1.1 \times 10^{-11}$ & $2.9 \times 10^{-9}$ & $6.2 \times 10^{-11}$ & $1.0 \times 10^{-8}$ & $2.1 \times 10^{-10}$ & $1.3 \times 10^{-8}$ & $2.8 \times 10^{-10}$ & $1 \times 10^{-8}$ \\
\hline 6303 & $3.0 \times 10^{-9}$ & $6.3 \times 10^{-11}$ & $6.8 \times 10^{-9}$ & $1.4 \times 10^{-10}$ & $1.8 \times 10^{-8}$ & $3.8 \times 10^{-10}$ & $2.8 \times 10^{-8}$ & $5.8 \times 10^{-10}$ & $3 \times 10^{-8}$ \\
\hline 6601 & $4.0 \times 10^{-11}$ & $8.5 \times 10^{-13}$ & - & - & $2.2 \times 10^{-9}$ & $4.6 \times 10^{-11}$ & $2.2 \times 10^{-9}$ & $4.7 \times 10^{-11}$ & $2 \times 10^{-9}$ \\
\hline
\end{tabular}

a The COPCs were not detected during the two quarters of joint sampling at SP-5101, SP-5402, SP-6306, and SP-6501.

b A hyphen (-) indicates that the parameter was not detected. 
TABLE 5.3 Estimated Radiological Carcinogenic Risks for the Current and Future Recreational Visitor

Estimated Risk

\begin{tabular}{cccc} 
& \multicolumn{2}{c}{ Uranium } \\
\cline { 2 - 3 } Spring & \multicolumn{2}{c}{ Dermal } & Total \\
\cline { 2 - 3 } ID & Ingestion & Den & \\
\hline & & & \\
5101 & $4.9 \times 10^{-9}$ & $5.1 \times 10^{-11}$ & $5 \times 10^{-9}$ \\
5201 & $1.1 \times 10^{-8}$ & $1.2 \times 10^{-10}$ & $1 \times 10^{-8}$ \\
5303 & $1.5 \times 10^{-6}$ & $1.6 \times 10^{-8}$ & $2 \times 10^{-6}$ \\
5402 & $1.2 \times 10^{-8}$ & $1.3 \times 10^{-10}$ & $1 \times 10^{-8}$ \\
5501 & $9.4 \times 10^{-9}$ & $9.9 \times 10^{-11}$ & $9 \times 10^{-9}$ \\
5504 & $6.4 \times 10^{-9}$ & $6.7 \times 10^{-11}$ & $6 \times 10^{-9}$ \\
5601 & $5.7 \times 10^{-9}$ & $6.0 \times 10^{-11}$ & $6 \times 10^{-9}$ \\
5602 & $4.2 \times 10^{-9}$ & $4.4 \times 10^{-11}$ & $4 \times 10^{-9}$ \\
5605 & $7.9 \times 10^{-9}$ & $8.3 \times 10^{-11}$ & $8 \times 10^{-9}$ \\
5612 & $6.6 \times 10^{-9}$ & $7.0 \times 10^{-11}$ & $7 \times 10^{-9}$ \\
6301 & $1.2 \times 10^{-6}$ & $1.2 \times 10^{-8}$ & $1 \times 10^{-6}$ \\
6303 & $1.7 \times 10^{-8}$ & $1.7 \times 10^{-10}$ & $2 \times 10^{-8}$ \\
6306 & $8.8 \times 10^{-9}$ & $9.3 \times 10^{-11}$ & $9 \times 10^{-9}$ \\
6501 & $3.6 \times 10^{-8}$ & $3.8 \times 10^{-10}$ & $4 \times 10^{-8}$ \\
6601 & $4.9 \times 10^{-9}$ & $5.1 \times 10^{-11}$ & $5 \times 10^{-9}$ \\
\hline
\end{tabular}


TABLE 5.4 Estimated Hazard Quotients for the Hypothetical Future Resident

\begin{tabular}{|c|c|c|c|c|c|c|c|c|c|}
\hline \multirow[b]{2}{*}{ Well ID } & \multicolumn{9}{|c|}{ Estimated Hazard Quotient } \\
\hline & Lithium & Molybdenum & $\begin{array}{l}\text { Total } \\
\end{array}$ & Nitrate $^{a}$ & $1,3,5-\mathrm{TNB}$ & 1,3-DNB & 2,4,6-TNT & 2,4-DNT & 2,6-DNT \\
\hline \multicolumn{10}{|l|}{ Deep Wells } \\
\hline MWD-05 & 0.027 & 0.0071 & 0.0078 & 0.0022 & $-b$ & - & - & - & - \\
\hline MWD-18 & 0.0092 & - & 0.011 & - & - & - & - & - & - \\
\hline MWGS-01 & - & - & - & - & - & - & - & - & - \\
\hline MWGS-02 & - & - & - & - & - & - & - & - & - \\
\hline MWS-18 & 0.013 & 0.042 & 0.021 & 0.0057 & - & - & - & - & - \\
\hline MWS-101 & 0.0015 & - & 0.0073 & - & - & - & - & - & - \\
\hline MWS-102 & 0.010 & 0.037 & 0.035 & - & - & - & - & - & - \\
\hline MWS-103 & 0.0058 & - & 0.010 & - & - & - & - & - & - \\
\hline TIL-3 & 0.0051 & 0.014 & 0.0015 & - & - & - & - & - & - \\
\hline \multicolumn{10}{|l|}{ Overburden } \\
\hline MW-2031 & - & - & - & - & - & - & - & - & - \\
\hline MW-2032 & 0.019 & 0.0099 & 0.057 & 0.96 & 2.7 & - & 0.37 & 0.0019 & 0.12 \\
\hline MW-2033 & 0.0051 & - & 0.032 & 0.019 & 2.5 & - & 0.066 & 0.0075 & 0.13 \\
\hline$M W-3001$ & - & - & - & - & - & - & - & - & - \\
\hline MW-3013 & - & - & - & - & - & - & - & - & - \\
\hline$M W-3018$ & - & - & - & - & - & - & - & - & - \\
\hline MW-3022 & - & - & - & - & - & - & - & - & - \\
\hline MWV-01 & 0.0032 & - & 0.056 & 0.026 & 0.021 & - & 0.60 & 0.0015 & 0.027 \\
\hline MWV-02 & 0.0041 & 0.0088 & 0.041 & 0.046 & - & - & 0.006 & 0.00081 & 0.0013 \\
\hline MWV-09 & 0.0096 & - & 0.0098 & 0.014 & 7.7 & 0.11 & 1.6 & 0.27 & 0.079 \\
\hline MWV-13 & 0.0044 & - & 0.020 & 0.026 & - & - & - & - & - \\
\hline MWV-16 & 0.0016 & 0.006 & 0.016 & 0.019 & 0.18 & - & 0.013 & - & 0.0019 \\
\hline MWV-17 & 0.00036 & - & 0.00083 & 0.036 & - & - & - & - & - \\
\hline MWV-18 & - & - & - & - & - & - & - & - & - \\
\hline MWV-22 & 0.0032 & 0.0027 & 0.012 & 0.058 & - & - & - & - & 0.0038 \\
\hline MWV-24R & 0.011 & 0.006 & 0.021 & 0.0060 & 1.7 & - & 0.060 & 0.0018 & 0.038 \\
\hline USGS-2A & - & - & - & - & - & - & - & - & - \\
\hline \multicolumn{10}{|l|}{ Unweathered } \\
\hline MW-2019 & 0.029 & 0.15 & 0.041 & - & - & - & - & - & - \\
\hline MW-2021 & 0.0052 & 0.042 & 0.012 & 0.000086 & - & - & - & - & - \\
\hline$M W-2022$ & 0.0051 & 0.013 & 0.017 & - & - & - & - & - & - \\
\hline$M W-2023$ & 0.0049 & 0.036 & 0.034 & - & - & - & - & - & - \\
\hline $\mathrm{MW}-2024$ & 0.0073 & 0.011 & 0.0015 & - & - & - & - & - & - \\
\hline MW-2025 & - & - & - & - & - & - & - & - & - \\
\hline MW-2026 & 0.0040 & 0.045 & 0.011 & - & - & - & - & - & - \\
\hline MW-2027 & 0.0056 & 0.018 & 0.011 & - & - & - & - & - & - \\
\hline MW-2028 & 0.026 & 0.026 & 0.017 & - & - & - & - & - & - \\
\hline MW-2029 & - & - & - & - & - & - & - & - & - \\
\hline MW-3002 & - & - & - & - & - & - & - & - & - \\
\hline MW-3006 & 0.017 & 0.079 & 0.0095 & - & - & - & - & - & - \\
\hline MW-3024 & 0.28 & - & 0.042 & 6.3 & - & - & - & 0.0018 & 0.012 \\
\hline MW-3026 & 0.048 & 0.012 & 0.058 & 3.8 & 0.077 & - & - & 0.00099 & 0.0013 \\
\hline MW-4004 & 0.0055 & 0.026 & 0.029 & 0.019 & - & - & - & - & - \\
\hline MW-4007 & 0.0082 & 0.032 & 0.024 & - & - & - & - & - & - \\
\hline MW-4008 & 0.0036 & - & 0.011 & - & - & - & - & - & - \\
\hline MW-4009 & 0.12 & 0.045 & 0.023 & 0.0024 & - & - & - & - & - \\
\hline MW-4011 & 0.089 & 0.018 & 0.043 & 2.9 & - & - & - & - & 0.0018 \\
\hline$M W-4012$ & 0.11 & 0.20 & 0.068 & - & - & - & - & - & - \\
\hline
\end{tabular}


TABLE 5.4 (Cont.)

Estimated Hazard Quotient

\begin{tabular}{|c|c|c|c|c|c|c|c|c|c|}
\hline \multirow[b]{2}{*}{ Well ID } & \multicolumn{9}{|c|}{ Estimated Hazard Quotient } \\
\hline & Lithium & Molybdenum & $\begin{array}{c}\text { Uranium, } \\
\text { Total }\end{array}$ & Nitrate $^{\mathbf{a}}$ & 1,3,5-TNB & 1,3-DNB & $2,4,6-\mathrm{TNT}$ & 2,4-DNT & 2,6-DNT \\
\hline \multicolumn{10}{|c|}{ Unweathered (cont.) } \\
\hline$M W-4022$ & 0.046 & 0.036 & 0.07 & 0.0067 & - & - & - & - & - \\
\hline MWD-02 & 0.042 & 0.044 & 0.035 & - & - & - & - & - & - \\
\hline MWD-06 & 0.0056 & - & 0.0079 & - & - & - & - & - & - \\
\hline MWD-09 & 0.0074 & - & 0.013 & 0.014 & 0.036 & - & - & 0.00058 & 0.0044 \\
\hline MWD-23 & 0.0059 & 0.13 & 0.068 & - & - & - & - & - & - \\
\hline MWD-106 & 0.0049 & - & - & - & - & - & - & - & - \\
\hline MWS-05 & 0.0041 & - & 0.014 & - & - & - & - & - & - \\
\hline MWS-06 & 0.0059 & - & 0.039 & - & - & - & - & - & - \\
\hline MWS-105 & 0.0027 & 0.024 & 0.0022 & - & - & - & - & - & - \\
\hline MWS-106 & 0.0032 & 0.013 & 0.016 & - & - & - & - & - & - \\
\hline MWS-109 & 0.0030 & 0.010 & 0.014 & - & - & - & - & - & - \\
\hline TIL-4 & - & - & - & - & - & - & - & - & - \\
\hline USGS-1 & 0.0052 & - & 0.014 & 0.014 & 0.035 & 0.038 & - & 0.00070 & 0.00060 \\
\hline USGS-6 & 0.0025 & 0.018 & 0.054 & 0.0094 & - & - & - & - & - \\
\hline \multicolumn{10}{|l|}{ Weathered } \\
\hline$M W-2001$ & 0.0036 & - & 0.0088 & 0.84 & 0.030 & - & - & 0.0018 & 0.0015 \\
\hline MW-2002 & 0.37 & 0.060 & 0.0065 & 2.2 & - & - & - & 0.00096 & 0.011 \\
\hline$M W-2003$ & 0.59 & 0.012 & 0.014 & 5.3 & - & - & - & 0.0021 & 0.012 \\
\hline MW-2004 & - & - & - & - & - & - & - & - & - \\
\hline MW-2005 & 0.13 & 0.0077 & 0.0062 & 1.1 & 0.019 & - & - & 0.00084 & 0.0025 \\
\hline MW-2006 & 0.022 & 0.013 & 0.0066 & 0.084 & - & - & - & 0.0019 & 0.00025 \\
\hline MW-2007 & 0.0055 & 0.023 & 0.014 & 0.050 & - & - & - & - & - \\
\hline MW-2008 & - & - & - & - & - & - & - & - & - \\
\hline MW-2009 & - & - & - & - & - & - & - & - & - \\
\hline $\mathrm{MW}-2010$ & 0.023 & 0.058 & 0.016 & 0.024 & 0.082 & - & 0.019 & 0.0013 & 0.021 \\
\hline MW-2011 & 0.0088 & - & 0.0040 & 0.082 & 0.22 & - & - & 0.0027 & 0.044 \\
\hline$M W-2012$ & 0.0025 & - & 0.0046 & 0.0091 & 0.77 & - & 0.025 & 0.0014 & 0.018 \\
\hline MW-2013 & 0.0084 & - & 0.0090 & 0.017 & 3.4 & - & 0.047 & 0.0049 & 0.012 \\
\hline MW-2014 & 0.028 & - & 0.0066 & 0.031 & 1.0 & 0.24 & 0.0024 & 0.0022 & 0.011 \\
\hline MW-2015 & 0.021 & - & 0.026 & 0.0091 & - & - & - & - & - \\
\hline$M W-2016$ & - & - & - & - & - & - & - & - & - \\
\hline$M W-2017$ & 0.15 & 0.085 & 0.16 & 0.094 & - & - & - & - & - \\
\hline MW-2018 & 0.027 & 0.025 & 0.021 & 0.011 & - & - & - & - & - \\
\hline MW-2020 & - & - & - & - & - & - & - & - & - \\
\hline MW-2030 & 0.0088 & - & 0.17 & 0.022 & 4.5 & - & 1.6 & 0.0034 & 0.30 \\
\hline MW-2034 & 0.044 & - & 0.041 & 0.082 & - & - & - & - & - \\
\hline$M W-2035$ & 0.0037 & 0.019 & 0.0055 & 0.011 & - & - & - & - & - \\
\hline$M W-2036$ & 0.0093 & - & 0.010 & 0.069 & - & - & - & - & - \\
\hline MW-2037 & 0.56 & - & 0.017 & 5.0 & 0.10 & - & - & 0.0077 & 0.0036 \\
\hline MW-2038 & 0.71 & 0.0077 & 0.020 & 15 & 0.13 & - & - & 0.023 & 0.0088 \\
\hline$M W-2039$ & 0.030 & 0.024 & 0.042 & 0.89 & 4.0 & - & - & 0.0016 & 0.047 \\
\hline MW-2040 & 0.045 & 0.036 & 0.041 & 3.9 & - & - & - & - & - \\
\hline MW-2041 & 0.035 & 0.012 & 0.046 & 5.1 & - & - & - & - & - \\
\hline MW-2042 & 0.028 & - & 0.035 & 0.096 & - & - & - & - & - \\
\hline$M W-2043$ & 0.023 & 0.0082 & 0.024 & 0.099 & - & - & - & 0.0012 & - \\
\hline MW-2044 & 0.039 & 0.0099 & 0.031 & 0.022 & - & - & - & - & - \\
\hline MW-2046 & - & - & - & - & - & - & - & - & - \\
\hline MW-3003 & 0.89 & 0.031 & 0.25 & 5.1 & - & - & - & 0.0023 & 0.0023 \\
\hline MW-3007 & - & - & - & - & - & - & - & - & - \\
\hline
\end{tabular}


TABLE 5.4 (Cont.)

Estimated Hazard Quotient

\begin{tabular}{|c|c|c|c|c|c|c|c|c|c|}
\hline Well ID & Lithium & Molybdenum & $\begin{array}{c}\text { Uranium, } \\
\text { Total }\end{array}$ & Nitrate $^{a}$ & $1,3,5$-TNB & 1,3-DNB & 2,4,6-TNT & 2,4-DNT & 2,6-DNT \\
\hline \multicolumn{10}{|c|}{ Weathered (cont.) } \\
\hline MW-3008 & - & - & - & - & - & - & - & - & - \\
\hline MW-3009 & - & - & - & - & - & - & - & - & - \\
\hline MW-3010 & - & - & - & - & - & - & - & - & - \\
\hline MW-3019 & 0.020 & 0.0055 & 0.029 & 0.021 & - & - & - & - & - \\
\hline MW-3023 & 0.88 & 1.4 & 0.17 & 3.6 & - & - & - & 0.068 & 0.14 \\
\hline MW-3025 & 0.22 & - & 0.038 & 8.9 & - & - & - & 0.0013 & - \\
\hline MW-3027 & 0.025 & - & 0.017 & 1.1 & 0.041 & - & - & 0.00079 & 0.0011 \\
\hline MW-4001 & 0.011 & 0.0099 & 0.0056 & 0.68 & 21 & - & 0.099 & 0.018 & 0.085 \\
\hline MW-4002 & 0.0053 & 0.0060 & 0.0082 & 0.089 & 0.034 & - & 0.099 & 0.0019 & 0.0079 \\
\hline MW-4003 & 0.0041 & - & 0.016 & 0.011 & - & - & - & - & - \\
\hline MW-4005 & 0.0092 & 0.027 & 0.022 & 0.027 & - & - & - & - & - \\
\hline MW-4006 & 0.0041 & 0.006 & 0.0036 & 0.024 & 10 & - & - & 0.0022 & 0.085 \\
\hline MW-4010 & 0.0079 & 0.020 & 0.042 & - & - & - & - & - & - \\
\hline MW-4013 & 0.093 & - & 0.016 & 1.6 & 15 & - & 0.0025 & 0.0011 & 0.020 \\
\hline MW-4014 & 0.0048 & - & 0.0030 & 0.099 & 0.060 & - & - & 0.00036 & 0.0024 \\
\hline$M W-4015$ & 0.0026 & 0.0014 & 0.0044 & 0.072 & 0.99 & - & - & 0.0026 & 0.030 \\
\hline$M W-4016$ & 0.0051 & 0.053 & 0.043 & 0.0068 & - & - & - & - & - \\
\hline MW-4017 & - & - & - & - & - & - & - & - & - \\
\hline MW-4018 & 0.0056 & - & 0.0087 & 0.046 & - & - & - & - & - \\
\hline MW-4019 & 0.014 & - & 0.023 & 0.0045 & - & - & - & - & - \\
\hline$M W-4020$ & 0.031 & 0.0071 & 0.13 & - & - & - & - & - & - \\
\hline MW-4021 & 0.031 & - & 0.042 & - & - & - & - & - & - \\
\hline$M W-4023$ & 0.020 & - & 0.021 & 0.045 & 0.048 & - & - & 0.00092 & 0.00063 \\
\hline MW-4024 & 0.11 & 0.061 & 0.82 & 0.024 & - & - & - & - & - \\
\hline MW-4025 & 0.016 & 0.023 & 0.014 & 0.019 & - & - & - & - & - \\
\hline MWD-15 & 0.0016 & - & 0.0067 & 0.0074 & - & - & - & - & 0.018 \\
\hline MWD-25 & 0.0033 & 0.010 & 0.024 & 0.0065 & - & - & - & - & - \\
\hline MWD-107 & 0.0074 & 0.025 & 0.028 & - & 0.026 & - & - & 0.00027 & 0.0025 \\
\hline MWD-112 & 0.0037 & 0.012 & 0.011 & - & - & - & - & - & - \\
\hline MWS-01 & 0.0026 & - & 0.018 & 0.043 & - & - & - & - & 0.018 \\
\hline MWS-02 & 0.0049 & 0.023 & 0.028 & 0.0017 & - & - & - & - & - \\
\hline MWS-03 & 0.0070 & 0.019 & 0.045 & - & - & - & - & - & - \\
\hline MWS-04 & 0.0055 & - & 0.14 & 0.15 & 6.0 & - & 0.066 & 0.0014 & 0.033 \\
\hline MWS-07 & - & - & 0.0099 & 0.039 & 9.9 & - & 0.14 & 0.00067 & 0.033 \\
\hline MWS-08 & 0.031 & - & 0.015 & 0.033 & - & - & - & - & - \\
\hline MWS-09 & 0.0090 & - & 0.016 & - & - & - & - & - & - \\
\hline MWS-10 & 0.0062 & 0.0093 & 0.0018 & 0.15 & 0.16 & - & 0.0015 & 0.0011 & 0.055 \\
\hline MWS-11 & 0.0033 & - & 0.023 & 0.15 & 0.020 & - & 0.0025 & 0.00075 & 0.015 \\
\hline MWS- 12 & 0.0042 & - & 0.014 & 0.05 & 1.0 & 0.074 & 0.0099 & 0.12 & 0.41 \\
\hline MWS-13 & 0.0093 & 0.0029 & 0.0073 & 0.021 & - & - & - & - & - \\
\hline MWS-14 & 0.034 & 0.0088 & 0.037 & 0.0031 & - & - & - & - & - \\
\hline MWS-15 & 0.0018 & - & 0.0076 & 0.016 & 0.99 & - & 0.32 & 0.0011 & 0.027 \\
\hline MWS-16 & - & - & 0.0089 & 0.13 & 5.5 & - & 0.16 & 0.0013 & 0.033 \\
\hline MWS-17 & 0.0036 & - & 0.016 & 0.053 & 0.038 & - & 0.0082 & 0.015 & 0.36 \\
\hline MWS-19 & 0.0016 & 0.0055 & 0.018 & 0.0026 & 0.028 & - & - & 0.0011 & 0.0038 \\
\hline MWS-20 & 0.0020 & - & 0.0094 & 0.096 & - & - & - & - & 0.00036 \\
\hline MWS-21 & 0.49 & 0.027 & 0.041 & 8.9 & - & - & - & 0.013 & 0.0047 \\
\hline MWS-22 & 0.0041 & 0.00070 & 0.016 & 0.051 & - & - & - & 0.00034 & 0.0036 \\
\hline MWS-24 & - & - & - & - & - & - & - & - & - \\
\hline MWS-25 & - & 0.0093 & 0.022 & 0.010 & - & - & - & - & - \\
\hline
\end{tabular}


TABLE 5.4 (Cont.)

Estimated Hazard Quotient

\begin{tabular}{|c|c|c|c|c|c|c|c|c|c|}
\hline Well ID & Lithium & Molybdenum & $\begin{array}{c}\text { Uranium, } \\
\text { Total } \\
\end{array}$ & Nitrate $^{\mathbf{a}}$ & 1,3,5-TNB & 1,3-DNB & 2,4,6-TNT & 2,4-DNT & 2,6-DNT \\
\hline \multicolumn{10}{|c|}{ Weathered (cont.) } \\
\hline MWS-26 & 0.021 & 0.011 & 0.054 & 0.0089 & - & - & - & - & - \\
\hline MWS-104 & 0.0023 & 0.024 & 0.018 & - & - & - & - & - & - \\
\hline MWS- 107 & 0.0068 & 0.0055 & 0.025 & 0.027 & 0.036 & - & - & 0.00081 & 0.0044 \\
\hline MWS-110 & 0.0045 & - & 0.0085 & 0.014 & 0.10 & - & - & - & 0.0015 \\
\hline MWS-112 & 0.023 & 0.19 & 0.037 & 0.0022 & - & - & - & 0.00077 & 0.00049 \\
\hline USGS-2 & - & 0.0099 & 0.000014 & - & - & - & - & - & - \\
\hline USGS-3 & 0.0066 & 0.0088 & 0.019 & 0.015 & 0.055 & - & - & 0.00030 & 0.0052 \\
\hline USGS-4 & 0.0053 & 0.0066 & 0.0073 & 0.026 & 0.99 & - & - & 0.021 & 0.058 \\
\hline USGS-5 & 0.0048 & 0.030 & 0.067 & 0.0039 & - & - & - & - & - \\
\hline USGS-7 & - & - & - & - & - & - & - & - & - \\
\hline USGS-8 & 0.014 & 0.0088 & 0.0085 & 0.055 & - & - & - & - & - \\
\hline USGS-9 & 0.0041 & $=$ & 0.0047 & 0.055 & - & $=$ & - & 0.0013 & 0.00041 \\
\hline
\end{tabular}

Estimated Hazard Quotient

\begin{tabular}{|c|c|c|c|c|c|c|c|c|}
\hline Well ID & $\begin{array}{l}\text { 2-Amino- } \\
\text { 4,6-DNT }\end{array}$ & $\begin{array}{l}\text { 4-Amino- } \\
\text { 2,6-DNT }\end{array}$ & $\begin{array}{c}\text { Nitro- } \\
\text { benzene }\end{array}$ & $\begin{array}{l}\text { o-Nitro- } \\
\text { toluene }\end{array}$ & $\begin{array}{c}\text { m-Nitro- } \\
\text { toluene }\end{array}$ & $\begin{array}{l}\text { p-Nitro- } \\
\text { toluene }\end{array}$ & 1,2-DCE & Total $^{\mathrm{c}}$ \\
\hline \multicolumn{9}{|l|}{ Deep Wells } \\
\hline MWD-05 & - & - & - & - & - & - & - & 0.04 \\
\hline MWD-18 & - & - & - & - & - & - & - & 0.02 \\
\hline MWGS-01 & - & - & - & - & - & - & - & - \\
\hline MWGS-02 & - & - & - & - & - & - & - & - \\
\hline MWS-18 & - & - & - & - & - & - & - & 0.08 \\
\hline MWS-101 & - & - & - & - & - & - & - & 0.009 \\
\hline MWS-102 & - & - & - & - & - & - & - & 0.08 \\
\hline MWS-103 & - & - & - & - & - & - & - & 0.02 \\
\hline TIL-3 & - & - & - & - & - & - & - & 0.02 \\
\hline \multicolumn{9}{|l|}{ Overburden } \\
\hline MW-2031 & - & - & - & - & - & - & - & - \\
\hline MW-2032 & 1.6 & 1.3 & - & 0.00058 & 0.00012 & - & 0.0053 & 7 \\
\hline MW-2033 & 1.6 & 1.5 & - & 0.0018 & 0.00013 & 0.00025 & - & 6 \\
\hline MW-3001 & - & - & - & - & - & - & - & - \\
\hline MW-3013 & - & - & - & - & - & - & - & - \\
\hline MW-3018 & - & - & - & - & - & - & - & - \\
\hline MW-3022 & - & - & - & - & - & - & - & - \\
\hline MWV-01 & 1.7 & 3.1 & - & 0.00044 & - & - & - & 6 \\
\hline MWV-02 & 0.23 & 0.46 & - & - & - & - & - & 0.8 \\
\hline MWV-09 & 16 & 12 & - & - & 0.00036 & 0.0006 & - & 40 \\
\hline$M W V-13$ & - & - & - & - & - & - & - & 0.05 \\
\hline MWV-16 & 0.15 & 0.26 & - & - & - & - & - & 0.7 \\
\hline MWV-17 & - & - & - & - & - & - & - & 0.04 \\
\hline MWV-18 & - & - & - & - & - & - & - & - \\
\hline MWV-22 & 0.026 & 0.096 & - & - & - & - & - & 0.2 \\
\hline MWV-24R & 0.21 & 0.46 & - & 0.00099 & - & 0.00017 & - & 3 \\
\hline USGS-2A & - & - & - & - & - & - & - & - \\
\hline
\end{tabular}


TABLE 5.4 (Cont.)

\begin{tabular}{|c|c|c|c|c|c|c|c|c|}
\hline \multirow[b]{2}{*}{ Well ID } & \multicolumn{8}{|c|}{ Estimated Hazard Quotient } \\
\hline & $\begin{array}{l}\text { 2-Amino- } \\
\text { 4,6-DNT }\end{array}$ & $\begin{array}{l}\text { 4-Amino- } \\
\text { 2,6-DNT }\end{array}$ & $\begin{array}{c}\text { Nitro- } \\
\text { benzene }\end{array}$ & $\begin{array}{l}\text { o-Nitro- } \\
\text { toluene }\end{array}$ & $\begin{array}{c}\text { m-Nitro- } \\
\text { toluene }\end{array}$ & $\begin{array}{l}\text { p-Nitro- } \\
\text { toluene }\end{array}$ & 1,2-DCE & Total $^{\mathrm{c}}$ \\
\hline \multicolumn{9}{|l|}{ Unweathered } \\
\hline MW-2019 & - & - & - & - & - & - & - & 0.2 \\
\hline MW-2021 & - & - & - & - & - & - & - & 0.06 \\
\hline MW-2022 & - & - & - & - & - & - & - & 0.04 \\
\hline MW-2023 & - & - & - & - & - & - & - & 0.08 \\
\hline MW-2024 & - & - & - & - & - & - & - & 0.02 \\
\hline MW-2025 & - & - & - & - & - & - & - & - \\
\hline MW-2026 & - & - & - & - & - & - & - & 0.06 \\
\hline MW-2027 & - & - & - & - & - & - & - & 0.04 \\
\hline MW-2028 & - & - & - & - & - & - & - & 0.07 \\
\hline MW-2029 & - & - & - & - & - & - & - & - \\
\hline$M W-3002$ & - & - & - & - & - & - & - & - \\
\hline MW-3006 & - & - & - & - & - & - & - & 0.1 \\
\hline MW-3024 & 0.015 & 0.026 & - & 0.00082 & 0.00011 & - & 0.00083 & 7 \\
\hline MW-3026 & 0.14 & 0.17 & - & - & - & - & - & 4 \\
\hline$M W-4004$ & - & - & - & - & - & - & - & 0.08 \\
\hline$M W-4007$ & - & 0.0078 & - & - & - & - & - & 0.07 \\
\hline$M W-4008$ & - & - & - & - & - & - & - & 0.02 \\
\hline MW-4009 & - & - & - & - & - & - & - & 0.2 \\
\hline$M W-4011$ & 0.35 & 0.82 & - & - & - & - & - & 4 \\
\hline$M W-4012$ & - & - & - & - & - & - & - & 0.4 \\
\hline$M W-4022$ & - & - & - & - & - & - & - & 0.2 \\
\hline MWD-02 & - & - & - & - & - & - & - & 0.1 \\
\hline MWD-06 & - & - & - & - & - & - & - & 0.01 \\
\hline MWD-09 & 0.043 & 0.23 & - & 0.00015 & - & - & - & 0.4 \\
\hline MWD-23 & - & - & - & - & - & - & - & 0.2 \\
\hline MWD-106 & - & - & - & - & - & - & - & 0.005 \\
\hline MWS-05 & - & - & - & - & - & - & - & 0.02 \\
\hline MWS-06 & - & - & - & - & - & - & - & 0.05 \\
\hline MWS-105 & - & - & - & - & - & - & - & 0.03 \\
\hline MWS-106 & - & - & - & - & - & - & - & 0.03 \\
\hline MWS-109 & - & - & - & - & - & - & - & 0.03 \\
\hline TIL-4 & - & - & - & - & - & - & - & - \\
\hline USGS-1 & 0.026 & 0.091 & - & - & - & - & - & 0.2 \\
\hline USGS-6 & - & - & - & - & - & - & - & 0.08 \\
\hline \multicolumn{9}{|l|}{ Weathered } \\
\hline$M W-2001$ & - & - & - & - & - & - & - & 0.9 \\
\hline MW-2002 & 0.38 & 0.42 & - & - & - & - & - & 3 \\
\hline$M W-2003$ & 0.082 & 0.21 & - & 0.00049 & - & - & - & 6 \\
\hline MW-2004 & - & - & - & - & - & - & - & - \\
\hline MW-2005 & 0.055 & 0.055 & - & - & - & - & - & 1 \\
\hline MW-2006 & - & - & 0.0023 & - & - & - & - & 0.1 \\
\hline$M W-2007$ & - & - & - & - & - & - & - & 0.09 \\
\hline MW-2008 & - & - & - & - & - & - & - & - \\
\hline MW-2009 & - & - & - & - & - & - & - & - \\
\hline MW-2010 & 0.33 & 0.37 & - & 0.00023 & - & - & - & 0.9 \\
\hline$M W-2011$ & 0.91 & 0.45 & - & 0.0006 & - & - & $\therefore$ & 2 \\
\hline MW-2012 & 0.14 & 0.17 & - & - & - & 0.00077 & - & 1 \\
\hline MW-2013 & 1.1 & 1.0 & - & 0.00071 & 0.00016 & - & 0.022 & 6 \\
\hline MW-2014 & 0.19 & 0.29 & - & 0.0006 & - & - & - & 2 \\
\hline
\end{tabular}


TABLE 5.4 (Cont.)

\begin{tabular}{|c|c|c|c|c|c|c|c|c|}
\hline \multirow[b]{2}{*}{ Well ID } & \multicolumn{8}{|c|}{ Estimated Hazard Quotient } \\
\hline & $\begin{array}{l}\text { 2-Amino- } \\
\text { 4,6-DNT }\end{array}$ & $\begin{array}{l}\text { 4-Amino- } \\
\text { 2,6-DNT }\end{array}$ & $\begin{array}{c}\text { Nitro- } \\
\text { benzene }\end{array}$ & $\begin{array}{l}\text { o-Nitro- } \\
\text { toluene }\end{array}$ & $\begin{array}{c}\text { m-Nitro- } \\
\text { toluene }\end{array}$ & $\begin{array}{l}\text { p-Nitro- } \\
\text { toluene }\end{array}$ & 1,2-DCE & Total $^{c}$ \\
\hline \multicolumn{9}{|c|}{ Weathered (cont.) } \\
\hline MW-2015 & - & - & - & - & - & - & - & 0.06 \\
\hline MW-2016 & - & - & - & - & - & - & - & - \\
\hline MW-2017 & - & - & - & - & - & - & - & 0.5 \\
\hline MW-2018 & - & - & - & - & - & - & - & 0.08 \\
\hline MW-2020 & - & - & - & - & - & - & - & - \\
\hline MW-2030 & 2.5 & 2.0 & - & 0.00016 & - & - & - & 10 \\
\hline MW-2034 & - & - & - & - & - & - & - & 0.2 \\
\hline MW-2035 & - & - & - & - & - & - & - & 0.04 \\
\hline MW-2036 & - & - & - & - & - & - & - & 0.09 \\
\hline MW-2037 & 0.050 & 0.05 & - & - & - & - & 0.03 & 6 \\
\hline MW-2038 & 0.18 & 0.21 & 0.0034 & 0.00071 & - & - & 0.034 & 20 \\
\hline MW-2039 & 0.91 & 0.73 & 0.0030 & 0.0017 & - & - & - & 7 \\
\hline MW-2040 & - & - & - & - & - & - & - & 4 \\
\hline$M W-2041$ & - & - & - & - & - & - & - & 5 \\
\hline MW-2042 & - & - & - & - & - & - & - & 0.2 \\
\hline MW-2043 & - & - & - & - & - & - & - & 0.2 \\
\hline MW-2044 & 0.010 & 0.015 & - & - & - & - & - & 0.1 \\
\hline MW-2046 & - & - & - & - & - & - & 0.0027 & 0.003 \\
\hline MW-3003 & - & 0.016 & - & 0.0014 & 0.00038 & 0.00041 & - & 6 \\
\hline$M W-3007$ & - & - & - & - & - & - & - & - \\
\hline MW-3008 & - & - & - & - & - & - & - & - \\
\hline MW-3009 & - & - & - & - & - & - & - & - \\
\hline MW-3010 & - & - & - & - & - & - & - & - \\
\hline MW-3019 & - & - & - & - & - & - & - & 0.08 \\
\hline MW-3023 & 0.078 & 0.15 & - & 0.15 & 0.012 & 0.0019 & - & 7 \\
\hline MW-3025 & - & 0.014 & - & 0.00055 & - & - & - & 9 \\
\hline $\mathrm{MW}-3027$ & 0.087 & 0.082 & - & - & - & - & - & 1 \\
\hline $\mathrm{MW}-4001$ & 7.3 & 10 & - & 0.0023 & - & - & - & 40 \\
\hline$M W-4002$ & 0.64 & 1.1 & - & - & - & - & - & 2 \\
\hline$M W-4003$ & 0.0073 & 0.013 & - & - & - & - & - & 0.05 \\
\hline MW-4005 & - & - & - & - & - & - & - & 0.09 \\
\hline MW-4006 & 0.82 & 1.1 & - & 0.0019 & 0.00088 & - & - & 10 \\
\hline $\mathrm{MW}-4010$ & - & - & - & - & - & - & - & 0.07 \\
\hline MW-4013 & 0.78 & 0.91 & - & - & - & - & - & 20 \\
\hline MW-4014 & 0.13 & 0.24 & - & - & - & - & - & 0.5 \\
\hline MW-4015 & 1.5 & 1.9 & - & 0.0003 & - & - & - & 5 \\
\hline$M W-4016$ & - & - & - & - & - & - & - & 0.1 \\
\hline$M W-4017$ & - & - & - & - & - & - & - & - \\
\hline MW-4018 & - & - & - & - & - & - & - & 0.06 \\
\hline MW-4019 & - & - & - & - & - & - & - & 0.04 \\
\hline$M W-4020$ & - & - & - & - & - & - & - & 0.2 \\
\hline$M W-4021$ & - & - & - & - & - & - & - & 0.07 \\
\hline MW-4023 & 0.017 & 0.023 & - & - & - & - & - & 0.2 \\
\hline MW-4024 & - & - & - & - & - & - & - & 1 \\
\hline$M W-4025$ & - & - & - & - & - & - & - & 0.07 \\
\hline MWD-15 & 0.32 & 0.78 & - & - & - & - & - & 1 \\
\hline MWD-25 & - & - & - & - & - & - & - & 0.04 \\
\hline MWD-107 & 0.018 & 0.23 & - & - & - & - & - & 0.3 \\
\hline MWD-112 & - & - & - & - & - & - & - & 0.03 \\
\hline
\end{tabular}


TABLE 5.4 (Cont.)

\begin{tabular}{|c|c|c|c|c|c|c|c|c|}
\hline \multirow[b]{2}{*}{ Well ID } & \multicolumn{8}{|c|}{ Estimated Hazard Quotient } \\
\hline & $\begin{array}{l}\text { 2-Amino- } \\
\text { 4,6-DNT }\end{array}$ & $\begin{array}{l}\text { 4-Amino- } \\
\text { 2,6-DNT }\end{array}$ & $\begin{array}{c}\text { Nitro- } \\
\text { benzene }\end{array}$ & $\begin{array}{l}\text { o-Nitro- } \\
\text { toluene }\end{array}$ & $\begin{array}{c}\text { m-Nitro- } \\
\text { toluene }\end{array}$ & $\begin{array}{l}\text { p-Nitro- } \\
\text { toluene }\end{array}$ & 1,2-DCE & Total $^{\mathrm{c}}$ \\
\hline \multicolumn{9}{|c|}{ Weathered (cont.) } \\
\hline MWS-01 & - & 0.020 & - & - & - & - & - & 0.1 \\
\hline MWS-02 & - & - & - & - & - & - & - & 0.06 \\
\hline MWS-03 & - & - & - & - & - & - & - & 0.07 \\
\hline MWS-04 & 3.5 & 3.8 & - & 0.00058 & - & - & - & 10 \\
\hline MWS-07 & 2.6 & 5.0 & - & - & - & - & - & 20 \\
\hline MWS-08 & - & - & - & - & - & - & - & 0.08 \\
\hline MWS-09 & - & - & - & - & - & - & - & 0.03 \\
\hline MWS-10 & 2.9 & 7.3 & - & 0.00027 & - & - & - & 10 \\
\hline MWS-11 & 0.22 & 1.1 & - & - & - & - & - & 2 \\
\hline MWS-12 & 0.5 & 1.1 & - & 0.27 & 0.021 & 0.082 & - & 4 \\
\hline MWS-13 & - & - & - & - & - & - & - & 0.04 \\
\hline MWS-14 & - & - & - & - & - & - & - & 0.08 \\
\hline MWS-15 & 5.0 & 9.1 & - & - & - & - & - & 20 \\
\hline MWS-16 & 2.1 & 3.8 & - & 0.00044 & - & - & - & 10 \\
\hline MWS-17 & 1.8 & 2.1 & - & 0.024 & 0.00085 & 0.0025 & - & 4 \\
\hline MWS-19 & 0.12 & 0.18 & - & - & - & - & - & 0.4 \\
\hline MWS-20 & - & 0.026 & - & - & - & - & - & 0.1 \\
\hline MWS-21 & 0.11 & 0.23 & - & 0.00041 & - & - & - & 10 \\
\hline MWS-22 & 0.033 & 0.087 & - & - & - & - & - & 0.2 \\
\hline MWS-24 & - & - & - & - & - & - & - & - \\
\hline MWS-25 & - & - & - & - & - & - & - & 0.04 \\
\hline MWS-26 & - & - & - & - & - & - & - & 0.1 \\
\hline MWS-104 & - & - & - & - & - & - & - & 0.04 \\
\hline MWS-107 & 0.027 & 0.30 & - & - & - & - & - & 0.4 \\
\hline MWS-110 & 0.082 & 0.17 & - & - & - & - & - & 0.4 \\
\hline MWS-112 & 0.026 & 0.064 & 0.0034 & - & - & - & - & 0.4 \\
\hline USGS-2 & - & - & - & - & - & - & - & 0.01 \\
\hline USGS-3 & 0.011 & 0.11 & - & - & - & - & - & 0.2 \\
\hline USGS-4 & 0.91 & 1.0 & - & 0.0027 & 0.0003 & - & - & 3 \\
\hline USGS-5 & - & - & - & - & - & - & - & 0.1 \\
\hline USGS-7 & - & - & - & - & - & - & - & - \\
\hline USGS-8 & - & - & - & - & - & - & - & 0.09 \\
\hline USGS-9 & 1.2 & 1.7 & - & - & - & - & - & 3 \\
\hline
\end{tabular}

a The hazard quotient for an infant from ingestion of nitrate ranges from 0.0005 (MW-2021) to 90 (MW-2038).

b A hyphen (-) indicates the parameter was not detected.

c All values in total column rounded to one significant figure. 
TABLE 5.5 Estimated Chemical Carcinogenic Risks to the Hypothetical Future Resident for the Ingestion Pathway

\begin{tabular}{|c|c|c|c|c|c|}
\hline \multirow[b]{2}{*}{ Well ID } & \multicolumn{5}{|c|}{ Estimated Risk } \\
\hline & 2,4,6-TNT & 2,4-DNT & 2,6-DNT & $\mathrm{TCE}^{\mathrm{a}}$ & Total $^{b}$ \\
\hline \multicolumn{6}{|l|}{ Deep Wells } \\
\hline MWD-05 & $--^{c}$ & - & - & - & - \\
\hline MWD-18 & - & - & - & - & - \\
\hline MWGS-01 & - & - & - & - & - \\
\hline MWGS-02 & - & - & - & - & - \\
\hline MWS-18 & - & - & - & - & - \\
\hline MWS-101 & - & - & - & - & - \\
\hline MWS-102 & - & - & - & - & - \\
\hline MWS-103 & - & - & - & - & - \\
\hline TIL-3 & - & - & - & - & - \\
\hline \multicolumn{6}{|l|}{ Overburden } \\
\hline MW-2031 & - & - & - & - & - \\
\hline MW-2032 & $2.4 \times 10^{-6}$ & $1.1 \times 10^{-6}$ & $3.5 \times 10^{-5}$ & $\begin{array}{c}8.1 \times 10^{-8} \\
\left(1.2 \times 10^{-7}\right)\end{array}$ & $\begin{array}{c}4 \times 10^{-5} \\
\left(2 \times 10^{-7}\right)\end{array}$ \\
\hline MW-2033 & $4.2 \times 10^{-7}$ & $4.4 \times 10^{-6}$ & $3.9 \times 10^{-5}$ & - & $4 \times 10^{-5}$ \\
\hline MW-3001 & - & - & - & - & - \\
\hline$M W-3013$ & - & - & - & - & - \\
\hline MW-3018 & - & - & - & - & - \\
\hline MW-3022 & - & - & - & - & - \\
\hline MWV-01 & $3.9 \times 10^{-6}$ & $8.8 \times 10^{-7}$ & $8.0 \times 10^{-6}$ & - & $1 \times 10^{-5}$ \\
\hline MWV-02 & $3.9 \times 10^{-8}$ & $4.7 \times 10^{-7}$ & $3.8 \times 10^{-7}$ & - & $9 \times 10^{-7}$ \\
\hline MWV-09 & $1.1 \times 10^{-5}$ & $1.6 \times 10^{-4}$ & $2.3 \times 10^{-5}$ & - & $2 \times 10^{-4}$ \\
\hline MWV-13 & - & - & - & - & - \\
\hline$M W V-16$ & $9.5 \times 10^{-8}$ & - & $5.5 \times 10^{-7}$ & - & $7 \times 10^{-7}$ \\
\hline MWV-17 & - & - & - & - & - \\
\hline MWV-18 & - & - & - & - & - \\
\hline MWV-22 & - & - & $1.1 \times 10^{-6}$ & - & $1 \times 10^{-6}$ \\
\hline MWV-24R & $3.9 \times 10^{-7}$ & $1.0 \times 10^{-6}$ & $1.1 \times 10^{-5}$ & - & $1 \times 10^{-5}$ \\
\hline USGS-2A & - & - & - & - & - \\
\hline \multicolumn{6}{|l|}{ Unweathered } \\
\hline MW-2019 & - & - & - & - & - \\
\hline MW-2021 & - & - & - & - & - \\
\hline MW-2022 & - & - & - & - & - \\
\hline MW-2023 & - & - & - & - & - \\
\hline MW-2024 & - & - & - & - & - \\
\hline MW-2025 & - & - & - & - & - \\
\hline MW-2026 & - & - & - & - & - \\
\hline MW-2027 & - & - & - & - & - \\
\hline MW-2028 & - & - & - & - & - \\
\hline MW-2029 & - & - & - & - & - \\
\hline MW-3002 & - & - & - & - & - \\
\hline MW-3006 & - & - & - & - & - \\
\hline MW-3024 & - & $1.0 \times 10^{-6}$ & $3.6 \times 10^{-6}$ & $\begin{array}{c}6.5 \times 10^{-6} \\
\left(9.8 \times 10^{-6}\right)\end{array}$ & $\begin{array}{r}5 \times 10^{-6} \\
\left(2 \times 10^{-5}\right)\end{array}$ \\
\hline
\end{tabular}


TABLE 5.5 (Cont.)

\begin{tabular}{|c|c|c|c|c|c|}
\hline \multirow[b]{2}{*}{ Well ID } & \multicolumn{5}{|c|}{ Estimated Risk } \\
\hline & 2,4,6-TNT & 2,4-DNT & 2,6-DNT & $\mathrm{TCE}^{\mathrm{a}}$ & Total $^{\mathrm{b}}$ \\
\hline \multicolumn{6}{|c|}{ Unweathered (cont.) } \\
\hline MW-3026 & - & $5.7 \times 10^{-7}$ & $3.7 \times 10^{-7}$ & - & $9 \times 10^{-7}$ \\
\hline MW-4004 & - & - & - & - & - \\
\hline$M W-4007$ & - & - & - & - & - \\
\hline MW-4008 & - & - & - & - & - \\
\hline$M W-4009$ & - & - & - & - & - \\
\hline$M W-4011$ & - & - & $5.2 \times 10^{-7}$ & - & $5 \times 10^{-7}$ \\
\hline MW-4012 & - & - & - & - & - \\
\hline$M W-4022$ & - & - & - & - & - \\
\hline MWD-02 & - & - & - & - & - \\
\hline MWD-06 & - & - & - & - & - \\
\hline MWD-09 & - & $3.4 \times 10^{-7}$ & $1.3 \times 10^{-6}$ & - & $2 \times 10^{-6}$ \\
\hline MWD-23 & - & - & - & - & - \\
\hline MWD-106 & - & - & - & - & - \\
\hline MWS-05 & - & - & - & - & - \\
\hline MWS-06 & - & - & - & - & - \\
\hline MWS-105 & - & - & - & - & - \\
\hline MWS-106 & - & - & - & - & - \\
\hline MWS-109 & - & - & - & - & - \\
\hline TIL-4 & - & - & - & - & - \\
\hline USGS-1 & - & $4.1 \times 10^{-7}$ & $1.8 \times 10^{-7}$ & - & $6 \times 10^{-7}$ \\
\hline USGS-6 & - & - & - & - & - \\
\hline \multicolumn{6}{|l|}{ Weathered } \\
\hline MW-2001 & - & $1.0 \times 10^{-6}$ & $4.5 \times 10^{-7}$ & - & $2 \times 10^{-6}$ \\
\hline$M W-2002$ & - & $5.6 \times 10^{-7}$ & $3.3 \times 10^{-6}$ & - & $4 \times 10^{-6}$ \\
\hline MW-2003 & - & $1.2 \times 10^{-6}$ & $3.6 \times 10^{-6}$ & - & $5 \times 10^{-6}$ \\
\hline MW-2004 & - & - & - & - & - \\
\hline MW-2005 & - & $4.9 \times 10^{-7}$ & $7.2 \times 10^{-7}$ & - & $1 \times 10^{-6}$ \\
\hline MW-2006 & - & $1.1 \times 10^{-6}$ & $7.2 \times 10^{-8}$ & - & $1 \times 10^{-6}$ \\
\hline MW-2007 & - & - & - & - & - \\
\hline MW-2008 & - & - & - & - & - \\
\hline MW-2009 & - & - & - & - & - \\
\hline MW-2010 & $1.2 \times 10^{-7}$ & $7.5 \times 10^{-7}$ & $6.0 \times 10^{-6}$ & - & $7 \times 10^{-6}$ \\
\hline MW-2011 & - & $1.6 \times 10^{-6}$ & $1.3 \times 10^{-5}$ & - & $2 \times 10^{-5}$ \\
\hline MW-2012 & $1.6 \times 10^{-7}$ & $7.9 \times 10^{-7}$ & $5.2 \times 10^{-6}$ & - & $6 \times 10^{-6}$ \\
\hline MW-2013 & $3.0 \times 10^{-7}$ & $2.9 \times 10^{-6}$ & $3.5 \times 10^{-5}$ & $\begin{array}{c}2.6 \times 10^{-7} \\
\left(3.9 \times 10^{-7}\right)\end{array}$ & $\begin{array}{r}4 \times 10^{-5} \\
\left(6 \times 10^{-7}\right)\end{array}$ \\
\hline MW-2014 & $1.5 \times 10^{-8}$ & $1.3 \times 10^{-6}$ & $3.3 \times 10^{-6}$ & - & $5 \times 10^{-6}$ \\
\hline MW-2015 & - & - & - & - & - \\
\hline MW-2016 & - & - & - & - & - \\
\hline MW-2017 & - & - & - & - & - \\
\hline MW-2018 & - & - & - & - & - \\
\hline MW-2020 & - & - & - & - & - \\
\hline MW-2030 & $1.0 \times 10^{-5}$ & $2.0 \times 10^{-6}$ & $8.8 \times 10^{-5}$ & - & $1 \times 10^{-4}$ \\
\hline MW-2034 & - & - & - & - & - \\
\hline
\end{tabular}


TABLE 5.5 (Cont.)

Estimated Risk

\begin{tabular}{|c|c|c|c|c|c|}
\hline Well ID & 2,4,6-TNT & 2,4-DNT & 2,6-DNT & $\mathrm{TCE}^{\mathrm{a}}$ & Total $^{b}$ \\
\hline \multicolumn{6}{|c|}{ Weathered (cont.) } \\
\hline MW-2035 & - & - & - & - & - \\
\hline MW-2036 & - & - & - & - & - \\
\hline MW-2037 & - & $4.5 \times 10^{-6}$ & $1.0 \times 10^{-6}$ & $\begin{array}{c}1.6 \times 10^{-4} \\
\left(2.4 \times 10^{-4}\right)\end{array}$ & $\begin{array}{c}6 \times 10^{-6} \\
\left(4 \times 10^{-4}\right)\end{array}$ \\
\hline MW-2038 & - & $1.4 \times 10^{-5}$ & $2.6 \times 10^{-6}$ & $\begin{array}{c}4.9 \times 10^{-4} \\
\left(7.4 \times 10^{-4}\right)\end{array}$ & $\begin{array}{c}2 \times 10^{-5} \\
\left(1 \times 10^{-3}\right)\end{array}$ \\
\hline MW-2039 & - & $9.6 \times 10^{-7}$ & $1.4 \times 10^{-5}$ & - & $2 \times 10^{-5}$ \\
\hline MW-2040 & - & - & - & - & - \\
\hline MW-2041 & - & - & - & - & - \\
\hline MW-2042 & - & - & - & - & - \\
\hline$M W-2043$ & - & $6.9 \times 10^{-7}$ & - & - & $7 \times 10^{-7}$ \\
\hline MW-2044 & - & - & - & - & - \\
\hline MW-3003 & - & $1.4 \times 10^{-6}$ & $6.8 \times 10^{-7}$ & - & $2 \times 10^{-6}$ \\
\hline MW-3007 & - & - & - & - & - \\
\hline MW-3008 & - & - & - & - & - \\
\hline MW-3009 & - & - & - & - & - \\
\hline MW-3010 & - & - & - & - & - \\
\hline MW-3019 & - & - & - & - & - \\
\hline MW-3023 & - & $4.0 \times 10^{-5}$ & $4.0 \times 10^{-5}$ & - & $8 \times 10^{-5}$ \\
\hline MW-3025 & - & $7.5 \times 10^{-7}$ & - & $\begin{array}{c}4.7 \times 10^{-6} \\
\left(7.1 \times 10^{-6}\right)\end{array}$ & $\begin{array}{c}8 \times 10^{-7} \\
\left(1 \times 10^{-5}\right)\end{array}$ \\
\hline MW-3027 & - & $4.6 \times 10^{-7}$ & $3.2 \times 10^{-7}$ & $\begin{array}{c}3.5 \times 10^{-7} \\
\left(5.2 \times 10^{-7}\right)\end{array}$ & $\begin{array}{c}8 \times 10^{-7} \\
\left(9 \times 10^{-7}\right)\end{array}$ \\
\hline MW-4001 & $6.3 \times 10^{-7}$ & $1.0 \times 10^{-5}$ & $2.5 \times 10^{-5}$ & $\begin{array}{c}5.2 \times 10^{-7} \\
\left(7.8 \times 10^{-7}\right)\end{array}$ & $\begin{array}{c}4 \times 10^{-5} \\
\left(1 \times 10^{-6}\right)\end{array}$ \\
\hline MW-4002 & $6.3 \times 10^{-7}$ & $1.1 \times 10^{-6}$ & $2.3 \times 10^{-6}$ & - & $4 \times 10^{-6}$ \\
\hline$M W-4003$ & - & - & - & - & - \\
\hline MW-4005 & - & - & - & - & - \\
\hline$M W-4006$ & - & $1.3 \times 10^{-6}$ & $2.5 \times 10^{-5}$ & - & $3 \times 10^{-5}$ \\
\hline MW-4010 & - & - & - & - & - \\
\hline MW-4013 & $1.6 \times 10^{-8}$ & $6.1 \times 10^{-7}$ & $5.9 \times 10^{-6}$ & - & $7 \times 10^{-6}$ \\
\hline MW-4014 & - & $2.1 \times 10^{-7}$ & $6.9 \times 10^{-7}$ & - & $9 \times 10^{-7}$ \\
\hline MW-4015 & - & $1.5 \times 10^{-6}$ & $8.8 \times 10^{-6}$ & - & $1 \times 10^{-5}$ \\
\hline MW-4016 & - & - & - & - & - \\
\hline MW-4017 & - & - & - & - & - \\
\hline MW-4018 & - & - & - & - & - \\
\hline MW-4019 & - & - & - & - & - \\
\hline$M W-4020$ & - & - & - & - & - \\
\hline$M W-4021$ & - & - & - & - & - \\
\hline$M W-4023$ & - & $5.3 \times 10^{-7}$ & $1.8 \times 10^{-7}$ & - & $7 \times 10^{-7}$ \\
\hline MW-4024 & - & - & - & - & - \\
\hline MW-4025 & - & - & - & - & - \\
\hline MWD-15 & - & - & $5.1 \times 10^{-6}$ & - & $5 \times 10^{-6}$ \\
\hline MWD-25 & - & - & - & - & - \\
\hline MWD-107 & - & $1.6 \times 10^{-7}$ & $7.2 \times 10^{-7}$ & - & $9 \times 10^{-7}$ \\
\hline MWD-112 & - & - & - & - & - \\
\hline
\end{tabular}


TABLE 5.5 (Cont.)

\begin{tabular}{|c|c|c|c|c|c|}
\hline \multirow[b]{2}{*}{ Well ID } & \multicolumn{5}{|c|}{ Estimated Risk } \\
\hline & 2,4,6-TNT & 2,4-DNT & 2,6-DNT & $\mathrm{TCE}^{\mathrm{a}}$ & Total $^{\mathrm{b}}$ \\
\hline \multicolumn{6}{|c|}{ Weathered (cont.) } \\
\hline MWS-01 & - & - & $5.1 \times 10^{-6}$ & - & $5 \times 10^{-6}$ \\
\hline MWS-02 & - & - & - & - & - \\
\hline MWS-03 & - & - & - & - & - \\
\hline MWS-04 & $4.2 \times 10^{-7}$ & $8.0 \times 10^{-7}$ & $9.6 \times 10^{-6}$ & - & $1 \times 10^{-5}$ \\
\hline MWS-07 & $9.2 \times 10^{-7}$ & $3.9 \times 10^{-7}$ & $9.6 \times 10^{-6}$ & - & $1 \times 10^{-5}$ \\
\hline MWS-08 & - & - & - & - & - \\
\hline MWS-09 & - & - & - & - & - \\
\hline MWS-10 & $9.9 \times 10^{-9}$ & $6.5 \times 10^{-7}$ & $1.6 \times 10^{-5}$ & - & $2 \times 10^{-5}$ \\
\hline MWS-11 & $1.6 \times 10^{-8}$ & $4.4 \times 10^{-7}$ & $4.3 \times 10^{-6}$ & - & $5 \times 10^{-6}$ \\
\hline MWS-12 & $6.3 \times 10^{-8}$ & $7.0 \times 10^{-5}$ & $1.2 \times 10^{-4}$ & - & $2 \times 10^{-4}$ \\
\hline MWS-13 & - & - & - & - & - \\
\hline MWS-14 & - & - & - & - & - \\
\hline MWS-15 & $2.1 \times 10^{-6}$ & $6.5 \times 10^{-7}$ & $8.0 \times 10^{-6}$ & - & $1 \times 10^{-5}$ \\
\hline MWS-16 & $1.0 \times 10^{-6}$ & $7.3 \times 10^{-7}$ & $9.6 \times 10^{-6}$ & - & $1 \times 10^{-5}$ \\
\hline MWS-17 & $5.3 \times 10^{-8}$ & $8.8 \times 10^{-6}$ & $1.0 \times 10^{-4}$ & - & $1 \times 10^{-4}$ \\
\hline MWS-19 & $\begin{array}{c}0.0 \times 10 \\
-\end{array}$ & $6.4 \times 10^{-7}$ & $1.1 \times 10^{-6}$ & - & $2 \times 10^{-6}$ \\
\hline MWS-20 & - & - & $1.0 \times 10^{-7}$ & - & $1 \times 10^{-7}$ \\
\hline MWS-21 & - & $7.5 \times 10^{-6}$ & $1.4 \times 10^{-6}$ & $\begin{array}{c}1.0 \times 10^{-4} \\
\left(1.6 \times 10^{-4}\right)\end{array}$ & $\begin{array}{r}9 \times 10^{-6} \\
\left(3 \times 10^{-4}\right)\end{array}$ \\
\hline MWS-22 & - & $2.0 \times 10^{-7}$ & $1.0 \times 10^{-6}$ & - & $1 \times 10^{-6}$ \\
\hline MWS-24 & - & - & - & - & - \\
\hline MWS-25 & - & - & - & - & - \\
\hline MWS-26 & - & - & - & - & - \\
\hline MWS-104 & - & - & - & - & - \\
\hline MWS- 107 & - & $4.7 \times 10^{-7}$ & $1.3 \times 10^{-6}$ & - & $2 \times 10^{-6}$ \\
\hline MWS-110 & - & - & $4.3 \times 10^{-7}$ & - & $4 \times 10^{-7}$ \\
\hline MWS-112 & - & $4.5 \times 10^{-7}$ & $1.4 \times 10^{-7}$ & - & $6 \times 10^{-7}$ \\
\hline USGS-2 & - & - & - & - & - \\
\hline USGS-3 & - & $1.8 \times 10^{-7}$ & $1.5 \times 10^{-6}$ & - & $2 \times 10^{-6}$ \\
\hline USGS-4 & - & $1.2 \times 10^{-5}$ & $1.7 \times 10^{-5}$ & - & $3 \times 10^{-5}$ \\
\hline USGS-5 & - & - & - & - & - \\
\hline USGS-7 & - & - & - & - & - \\
\hline USGS-8 & - & - & - & - & - \\
\hline USGS-9 & - & $7.3 \times 10^{-7}$ & $1.2 \times 10^{-7}$ & - & $9 \times 10^{-7}$ \\
\hline
\end{tabular}

a Risk from inhalation was also calculated for TCE because it is a volatile compound. Inhalation risks are shown in parentheses under the ingestion entries.

b Total values in parentheses indicate contribution from TCE.

c A hyphen $(-)$ indicates that the compound was not detected. 
TABLE 5.6 Estimated Radiological Carcinogenic Risks for the Hypothetical Future Resident ${ }^{\mathrm{a}}$

\begin{tabular}{|c|c|c|c|}
\hline Well ID & Uranium Risk & Well ID & Uranium Risk \\
\hline Deep Wells & \multicolumn{3}{|c|}{ Unweathered (cont.) } \\
\hline MWD-05 & $6 \times 10^{-7}$ & $M W-4004$ & $2 \times 10^{-6}$ \\
\hline MWD-18 & $9 \times 10^{-7}$ & MW-4007 & $2 \times 10^{-6}$ \\
\hline MWGS-01 & $-b$ & MW-4008 & $9 \times 10^{-7}$ \\
\hline MWGS-02 & - & MW-4009 & $2 \times 10^{-6}$ \\
\hline MWS-18 & $2 \times 10^{-6}$ & MW-4011 & $4 \times 10^{-6}$ \\
\hline MWS-101 & $6 \times 10^{-7}$ & MW-4012 & $6 \times 10^{-6}$ \\
\hline MWS-102 & $3 \times 10^{-6}$ & MW-4022 & $6 \times 10^{-6}$ \\
\hline MWS-103 & $9 \times 10^{-7}$ & MWD-02 & $3 \times 10^{-6}$ \\
\hline \multirow[t]{2}{*}{ TIL-3 } & $1 \times 10^{-7}$ & MWD-06 & $7 \times 10^{-7}$ \\
\hline & & MWD-09 & $1 \times 10^{-6}$ \\
\hline Overburden & & MWD-23 & $7 \times 10^{-6}$ \\
\hline MW-2031 & - & MWD-106 & - \\
\hline MW-2032 & $5.4 \times 10^{-6}$ & MWS-05 & $1 \times 10^{-6}$ \\
\hline MW-2033 & $3 \times 10^{-6}$ & MWS-06 & $3 \times 10^{-6}$ \\
\hline MW-3001 & - & MWS-105 & $2 \times 10^{-7}$ \\
\hline MW-3013 & - & MWS-106 & $1 \times 10^{-6}$ \\
\hline MW-3018 & - & MWS-109 & $1 \times 10^{-6}$ \\
\hline MW-3022 & - & TIL-4 & - \\
\hline MWV-01 & $5 \times 10^{-6}$ & USGS- 6 & $5 \times 10^{-6}$ \\
\hline MWV-02 & $3 \times 10^{-6}$ & & \\
\hline MWV-09 & $8 \times 10^{-7}$ & Weathered & \\
\hline MWV-13 & $2 \times 10^{-6}$ & MW-2001 & $2 \times 10^{-6}$ \\
\hline MWV-16 & $1 \times 10^{-6}$ & MW-2002 & $2 \times 10^{-6}$ \\
\hline MWV-17 & $7 \times 10^{-8}$ & MW-2003 & $2 \times 10^{-6}$ \\
\hline MWV-18 & - & MW-2004 & - \\
\hline MWV-22 & $1 \times 10^{-6}$ & MW-2005 & $6 \times 10^{-7}$ \\
\hline MWV-24R & $2 \times 10^{-6}$ & MW-2006 & $4 \times 10^{-7}$ \\
\hline \multirow[t]{2}{*}{ USGS-2A } & - & USGS-1 & $1 \times 10^{-6}$ \\
\hline & & MW-2007 & $1 \times 10^{-6}$ \\
\hline Unweathered & & MW-2008 & - \\
\hline MW-2019 & $3 \times 10^{-6}$ & MW-2009 & - \\
\hline MW-2021 & $1 \times 10^{-6}$ & $M W-2010$ & $1 \times 10^{-6}$ \\
\hline MW-2022 & $1 \times 10^{-6}$ & MW-2011 & $3 \times 10^{-7}$ \\
\hline MW-2023 & $3 \times 10^{-6}$ & $M W-2012$ & $4 \times 10^{-7}$ \\
\hline MW-2024 & $1 \times 10^{-7}$ & $M W-2013$ & $4 \times 10^{-6}$ \\
\hline MW-2025 & - & MW-2014 & $5 \times 10^{-7}$ \\
\hline MW-2026 & $9 \times 10^{-7}$ & MW-2015 & $2 \times 10^{-6}$ \\
\hline MW-2027 & $1 \times 10^{-6}$ & MW-2016 & - \\
\hline $\mathrm{MW}-2028$ & $1 \times 10^{-6}$ & $M W-2017$ & $1 \times 10^{-5}$ \\
\hline MW-2029 & - & MW-2018 & $2 \times 10^{-6}$ \\
\hline MW-3002 & - & MW-2020 & - \\
\hline MW-3006 & $8 \times 10^{-7}$ & $M W-2030$ & $1 \times 10^{-5}$ \\
\hline MW-3024 & $3 \times 10^{-6}$ & MW-2034 & $3 \times 10^{-6}$ \\
\hline MW-3026 & $5 \times 10^{-6}$ & MW-2035 & $5 \times 10^{-7}$ \\
\hline
\end{tabular}


TABLE 5.6 (Cont.)

\begin{tabular}{|c|c|c|c|}
\hline Well ID & Uranium Risk $^{\mathrm{a}}$ & Well ID & Uranium Risk $^{\mathrm{a}}$ \\
\hline Weathered (cont.) & \multicolumn{3}{|c|}{ Weathered (cont.) } \\
\hline MW-2036 & $9 \times 10^{-7}$ & MWD-25 & $2 \times 10^{-6}$ \\
\hline MW-2037 & $1 \times 10^{-6}$ & MWD-107 & $2 \times 10^{-6}$ \\
\hline MW-2038 & $2 \times 10^{-6}$ & MWD-112 & $9 \times 10^{-7}$ \\
\hline MW-2039 & $4 \times 10^{-6}$ & MWS-01 & $1 \times 10^{-6}$ \\
\hline MW-2040 & $3 \times 10^{-6}$ & MWS-02 & $2 \times 10^{-6}$ \\
\hline MW-2041 & $4 \times 10^{-6}$ & MWS-03 & $4 \times 10^{-6}$ \\
\hline MW-2042 & $3 \times 10^{-6}$ & MWS-04 & $1 \times 10^{-5}$ \\
\hline MW-2043 & $2 \times 10^{-6}$ & MWS-07 & $9 \times 10^{-7}$ \\
\hline MW-2044 & $3 \times 10^{-6}$ & MWS-08 & $1 \times 10^{-6}$ \\
\hline MW-3003 & $2 \times 10^{-5}$ & MWS-09 & $1 \times 10^{-6}$ \\
\hline MW-3007 & - & MWS-10 & $2 \times 10^{-7}$ \\
\hline MW-3008 & - & MWS-11 & $2 \times 10^{-6}$ \\
\hline MW-3009 & - & MWS-12 & $1 \times 10^{-6}$ \\
\hline MW-3010 & - & MWS-13 & $6 \times 10^{-7}$ \\
\hline MW-3019 & $2 \times 10^{-6}$ & MWS-14 & $3 \times 10^{-6}$ \\
\hline MW-3023 & $1 \times 10^{-5}$ & MWS-15 & $6 \times 10^{-7}$ \\
\hline MW-3025 & $3 \times 10^{-6}$ & MWS-16 & $7 \times 10^{-7}$ \\
\hline MW-3027 & $1 \times 10^{-6}$ & MWS-17 & $1 \times 10^{-6}$ \\
\hline MW-4001 & $5 \times 10^{-7}$ & MWS-19 & $1 \times 10^{-6}$ \\
\hline$M W-4002$ & $7 \times 10^{-7}$ & MWS-20 & $8 \times 10^{-7}$ \\
\hline$M W-4003$ & $2 \times 10^{-6}$ & MWS-21 & $3 \times 10^{-6}$ \\
\hline MW-4005 & $2 \times 10^{-6}$ & MWS-22 & $1 \times 10^{-6}$ \\
\hline MW-4006 & $3 \times 10^{-7}$ & MWS-24 & \\
\hline MW-4010 & $3 \times 10^{-6}$ & MWS-25 & $2 \times 10^{-6}$ \\
\hline MW-4013 & $1 \times 10^{-6}$ & MWS-26 & $5 \times 10^{-6}$ \\
\hline$M W-4014$ & $3 \times 10^{-7}$ & MWS-104 & $1 \times 10^{-6}$ \\
\hline MW-4015 & $4 \times 10^{-7}$ & MWS-107 & $2 \times 10^{-6}$ \\
\hline MW-4016 & $4 \times 10^{-6}$ & MWS-110 & $7 \times 10^{-7}$ \\
\hline MW-4017 & - & MWS- 112 & $3 \times 10^{-6}$ \\
\hline MW-4018 & $7 \times 10^{-7}$ & USGS-2 & $4 \times 10^{-7}$ \\
\hline MW-4019 & $2 \times 10^{-6}$ & USGS-3 & $2 \times 10^{-6}$ \\
\hline MW-4020 & $1 \times 10^{-5}$ & USGS-4 & $6 \times 10^{-7}$ \\
\hline$M W-4021$ & $4 \times 10^{-6}$ & USGS-5 & $6 \times 10^{-6}$ \\
\hline$M W-4023$ & $2 \times 10^{-6}$ & USGS-7 & - \\
\hline MW-4024 & $7 \times 10^{-5}$ & USGS-8 & $7 \times 10^{-7}$ \\
\hline$M W-4025$ & $1 \times 10^{-6}$ & USGS-9 & $4 \times 10^{-7}$ \\
\hline MWD-15 & $6 \times 10^{-7}$ & & \\
\hline
\end{tabular}

a Maximum uranium concentrations from the 1995 joint DOE/DA sampling rounds were used as EPCs.

b A hyphen (-) indicates samples were not collected as part of joint sampling rounds. 


\section{ECOLOGICAL RISK CHARACTERIZATION}

\subsection{METHODOLOGY}

Risks to biota were estimated by two methods: (1) determining an ecological effects quotient (EEQ) and (2) evaluating all available lines of evidence in a weight-of-evidence approach. For both approaches, the ecological significance of the potential risks was also considered and incorporated into the final risk characterization.

\subsubsection{Ecological Effects Quotient}

\subsubsection{Calculation}

For aquatic biota, the EEQ was estimated for each contaminant as the ratio between the exposure point concentration and a "safe" media concentration. For terrestrial biota, the EEQ for each contaminant was estimated as the ratio between the modeled ADD and a safe benchmark dose value. In both cases, values of the EEQ may vary from 0 to infinity, and values greater than 1.0 are considered to demonstrate a potential risk to the receptor from a particular contaminant. Values between 1.0 and 10 indicate a low risk, values between 10 and 50 indicate a moderate risk, values between 50 and 100 indicate a high risk, and values greater than 100 indicate extreme risk.

\subsubsection{Benchmark Values}

Estimating the EEQ requires the use of benchmark values that represent contaminant concentrations considered to be acceptable ("safe") to biota. Benchmark values are contaminantspecific and species-specific, typically represent NOAEL concentrations, and may include media concentrations, food concentrations, tissue concentrations, or dose estimates. For aquatic biota, surface water contaminant benchmark values used in this analysis included EPA ambient water quality criteria (chronic values), EPA ecotox threshold values (EPA 1996a), and values obtained from the literature (Suter and Tsao 1996; Talmage and Opresko 1996). For sediment-based contaminants, benchmark values were obtained from the National Oceanic and Atmospheric Administration, the EPA, and the open scientific literature. For terrestrial biota, EEQ values were estimated using contaminant-specific and species-specific NOAEL or LOAEL benchmark values obtained from the literature (Sample et al. 1996; Talmage and Opresko 1996). The benchmark values used for this risk assessment are presented in Table 6.1. 
TABLE 6.1 Benchmark Values Used to Estimate EEQs for Aquatic and Terrestrial Biota

\begin{tabular}{|c|c|c|c|c|c|}
\hline \multirow{3}{*}{\multicolumn{2}{|c|}{ Contaminant }} & \multicolumn{4}{|c|}{ Benchmark Value } \\
\hline & & \multicolumn{2}{|c|}{ Aquatic Biota } & \multirow[b]{2}{*}{$\begin{array}{c}\text { American Robin }{ }^{b} \\
(\mathrm{mg} / \mathrm{kg}-\mathrm{d})\end{array}$} & \multirow[b]{2}{*}{$\begin{array}{l}\text { White-Tailed } \\
\text { Deer }^{b} \\
\text { (mg/kg-d) }\end{array}$} \\
\hline & & $\begin{array}{c}\text { Surface Water }{ }^{2} \\
(\mu \mathrm{g} / \mathrm{L})\end{array}$ & $\begin{array}{l}\text { Sediment } \\
(\mathrm{mg} / \mathrm{kg})\end{array}$ & & \\
\hline \multicolumn{6}{|c|}{ Metals } \\
\hline & Antimony & $\mathrm{NC}^{\mathrm{c}}$ & NC & $\mathrm{NBA}^{\mathrm{c}}$ & 0.019 \\
\hline & Arsenic & NC & $8.2^{\mathrm{d}}$ & 2.46 & 0.019 \\
\hline & Cadmium & NC & $1.2^{\mathrm{d}}$ & 1.45 & 0.271 \\
\hline & Iron & 1,000 (chronic) & $\mathrm{NC}$ & NBA & NBA \\
\hline & Lead & $\mathrm{NC}$ & $47^{\mathrm{d}}$ & 3.85 & 2.24 \\
\hline & Lithium & $\mathrm{NC}$ & NBA & NBA & 1.8 \\
\hline & Manganese & $120^{\mathrm{e}}$ & $300^{f}$ & 997 & 25.0 \\
\hline & Mercury & 1.3 (chronic) & $0.15^{\mathrm{d}}$ & 0.064 & 0.009 \\
\hline & Molybdenum & $\mathrm{NC}$ & NBA & 3.5 & 0.04 \\
\hline & Nickel & NC & $21.0^{\mathrm{d}}$ & 77.4 & 11.2 \\
\hline & Selenium & NC & NBA & 0.5 & 0.056 \\
\hline & Silver & $\mathrm{NC}$ & $1.0^{\mathrm{f}}$ & $165^{g}$ & $5.54^{\mathrm{h}}$ \\
\hline & Strontium & NC & $\mathrm{NC}$ & $82.8^{\mathrm{i}}$ & 74.0 \\
\hline & Uranium, total & $570^{\mathrm{j}}$ & NBA & 16.0 & 0.46 \\
\hline \multicolumn{6}{|c|}{ Inorganic anion } \\
\hline & Nitrate-N & $90,000^{k}$ & NBA & NBA & 178 \\
\hline \multicolumn{6}{|c|}{ Nitroaromatic compounds } \\
\hline & $1,3,5-\mathrm{TNB}$ & 14.0 (chronic) $^{l}$ & $0.30^{l}$ & NBA & $0.9^{\mathrm{m}}$ \\
\hline & 1,3-DNB & NC & $1.2^{l}$ & NBA & $0.03^{\mathrm{m}}$ \\
\hline & $2,4,6-\mathrm{TNT}$ & 130 (chronic) $^{l}$ & $13^{l}$ & NBA & $0.4^{\mathrm{m}}$ \\
\hline & 2,4-DNT & NC & NBA & NBA & NBA \\
\hline & 2,6-DNT & NBA & NBA & NBA & NBA \\
\hline & 2-Amino-4,6-DNT & 0.02 & NBA & NBA & NBA \\
\hline & 4-Amino-2,6-DNT & NBA & NBA & NBA & NBA \\
\hline & Nitrotoluene & NBA & NBA & NBA & NBA \\
\hline \multicolumn{6}{|c|}{ Benchmark values are EPA (1986) ambient water quality criteria (AWQC), unless otherwise noted. } \\
\hline b & \multicolumn{5}{|c|}{ Benchmark values are NOAEL toxicological benchmarks developed by Sample et al. (1996), unless otherwise noted. } \\
\hline \multicolumn{6}{|c|}{$\mathrm{NBA}=$ no benchmark value available $; \mathrm{NC}=$ not a contaminant of ecological concern for the indicated medium. } \\
\hline \multicolumn{6}{|c|}{ Based on EPA ecotox threshold value (EPA 1996a). } \\
\hline \multicolumn{6}{|c|}{ Based on chronic value developed by Suter and Tsao (1996). } \\
\hline \multicolumn{6}{|c|}{ Based on value reported in Hull and Suter (1994). } \\
\hline \multicolumn{6}{|c|}{ g Based on data from Jensen et al. (1974). } \\
\hline \multicolumn{6}{|c|}{ h Based on data from Agency for Toxic Substances and Disease Registry (1990). } \\
\hline \multicolumn{6}{|c|}{ Based on data from Weber et al. (1968). } \\
\hline j & \multicolumn{5}{|c|}{ No AWQC available; value is lowest concentration reported as chemotoxic to aquatic biota (Poston et al. 1984). } \\
\hline k & \multicolumn{5}{|c|}{ EPA (1986) identifies the concentration as a potentially "safe" maximum concentration; no AWQC available. } \\
\hline \multicolumn{6}{|c|}{ Based on chronic value developed by Talmage and Opresko (1996). } \\
\hline $\mathrm{m}$ & Based on NOAEL $v$ & ped by Talmage a & ko (1996). & & \\
\hline
\end{tabular}




\subsubsection{Weight of Evidence}

The potential for adverse impacts to ecological resources was characterized using a weightof-evidence approach (EPA 1992b). In this approach, the EEQ risk estimates were evaluated together with the results of the biotic surveys and media-based toxicity tests. The potential for risks to ecological resources at the site was based on the frequency that the results of these various evaluations indicated actual or predicted adverse ecological effects and the degree of confidence in these results. Thus, the potential for unacceptable risks to ecological resources is greater if the results indicate a greater frequency for adverse effects and if the degree of confidence in the results is greater. Finally, the risk determination was evaluated with regard to its overall significance to the ecological resources of the area, and a final overall risk characterization was developed for the springs.

\subsection{RISK ESTIMATION AND ECOLOGICAL SIGNIFICANCE}

\subsubsection{Risk Estimation}

\subsubsection{Ecological Effects Quotient}

For aquatic biota, the EEQs were calculated by comparing the EPCs in surface water and sediment with suitable benchmark values; these EEQ values are presented in Table 6.2. The EEQ values were estimated for only those surface water and sediment contaminants that were identified as COECs (Tables 2.3 and 2.4) and for which appropriate chronic benchmark values were available (Table 6.1). A high risk (EEQ = 66) was identified for mercury. However, this high risk estimate is due primarily to use of the maximum reported mercury concentration in calculating the $95 \% \mathrm{UCL}$ EPC. For mercury, the EPC incorporated a concentration of $6,100 \mu \mathrm{g} / \mathrm{L}$ reported from spring SP-6303. This is the highest mercury concentration reported from any of the springs and likely represents an outlier; the next highest reported spring concentration is $340 \mu \mathrm{g} / \mathrm{L}$. Excluding the $6,100 \mu \mathrm{g} / \mathrm{L}$ mercury concentration from the risk estimation reduces the $95 \%$ UCL for mercury and results in a determination of low risk for mercury $(E E Q=10)$.

Similarly, the low EEQ risk level for iron was estimated using the maximum reported iron concentration, which also appears to be an outlier. This concentration, $400,000 \mu \mathrm{g} / \mathrm{L}$, was reported from a single spring (SP-6303) and is the highest reported from any of the springs. The next highest iron concentration is $7,300 \mu \mathrm{g} / \mathrm{L}$, which is 54 times lower than the highest reported concentration. Using the $95 \%$ UCL iron concentration (excluding the $400,000 \mu \mathrm{g} / \mathrm{L}$ concentration) results in a determination of no risk from iron (EEQ $=0.86$ ). No high risks (EEQ values between 50 and 100) were identified for any surface water or sediment contaminants, whereas a moderate risk was identified only for manganese $(E E Q=13)$ in surface water. Low risks or no risks $(E E Q<10)$ were identified for the sediment contaminants (Table 6.2). These results suggest that although 
TABLE 6.2 Estimated EEQs for Aquatic Biota Exposed to Surface Water and Sediment at Burgermeister Spring

\begin{tabular}{|c|c|c|c|c|c|c|}
\hline \multirow[b]{2}{*}{ Contaminant ${ }^{a}$} & \multicolumn{3}{|c|}{ Surface Water } & \multicolumn{3}{|c|}{ Sediment } \\
\hline & $\begin{array}{l}\mathrm{EPC}^{\mathrm{b}} \\
(\mu \mathrm{g} / \mathrm{L})\end{array}$ & EEQ & Risk Level $^{\mathrm{c}}$ & $\begin{array}{l}\mathrm{EPC}^{\mathrm{b}} \\
(\mu \mathrm{g} / \mathrm{L})\end{array}$ & EEQ & Risk Level $^{\mathrm{c}}$ \\
\hline \multicolumn{7}{|l|}{ Metals } \\
\hline Arsenic & $N C^{d}$ & $\mathrm{NA}^{\mathrm{e}}$ & NA & $43^{f}$ & 5.2 & Low risk \\
\hline Iron & 6,200 & 6.2 & Low risk & $\mathrm{NC}$ & NA & NA \\
\hline Lead & $\mathrm{NC}$ & NA & $\mathrm{NA}$ & $110^{\mathrm{f}}$ & 2.3 & Low risk \\
\hline Manganese & 1,600 & 13 & Moderate risk & NC & NA & NA \\
\hline Mercury & 86 & 66 & High risk & $\mathrm{NC}$ & NA & NA \\
\hline Selenium & $\mathrm{NC}$ & NA & NA & $0.96^{\mathrm{f}}$ & $\mathrm{NBA}^{\mathrm{d}}$ & NA \\
\hline Silver & $\mathrm{NC}$ & NA & NA & $1.7^{\mathrm{f}}$ & 1.7 & Low risk \\
\hline Uranium, total & 84 & 1.5 & Low risk & $100^{f}$ & NBA & NA \\
\hline \multicolumn{7}{|c|}{ Nitroaromatic compounds } \\
\hline 1,3-DNB & 0.033 & 0.01 & No risk & $\mathrm{NC}$ & $\mathrm{NA}$ & NA \\
\hline \multicolumn{7}{|c|}{$\begin{array}{l}\text { Included are only those contaminants identified as COECs (see Section } 2.2 \text { ) and for which a } \\
\text { benchmark value was available (Table 6.1). }\end{array}$} \\
\hline \multicolumn{7}{|c|}{ EPC values are the estimated $95 \%$ UCL value, unless otherwise noted (footnote $f$ ). } \\
\hline \multicolumn{7}{|c|}{$\begin{array}{l}\text { EEQ values greater than } 1.0 \text { are considered to demonstrate a potential risk to the receptor from a } \\
\text { particular contaminant. Values between } 1.0 \text { and } 10 \text { indicate a low risk, values between } 10 \text { and } 50 \\
\text { indicate a moderate risk, values between } 50 \text { and } 100 \text { indicate a high risk, and values greater than } 100 \\
\text { indicate extreme risk. }\end{array}$} \\
\hline \multicolumn{7}{|c|}{ d $\mathrm{NBA}=$ no benchmark available to estimate $\mathrm{EEQ} ; \mathrm{NC}=$ not a $\mathrm{COEC}$ for the indicated medium. } \\
\hline \multicolumn{7}{|c|}{ NA = not applicable. } \\
\hline f EPC values ar & $\mathrm{mu}$ & es & ra & & & \\
\hline
\end{tabular}

concentrations of some contaminants might adversely affect aquatic biota, the risks of unacceptable impacts are low.

The EEQ values for terrestrial biota (American robin and white-tailed deer) were calculated using modeled contaminant doses from water ingestion; the EEQ values are presented in Table 6.3. Uptake modeling was performed and EEQ values were estimated for all contaminants detected in spring water from all springs at concentrations exceeding background levels and for which benchmark values were available (Table 6.1). Except for the values calculated for mercury, all EEQ estimates were below 0.05 for both modeled receptor species (typically less than 0.01 ), indicating that current concentrations of contaminants in surface water at the spring pose no risk to terrestrial receptors that use the spring for drinking water. For mercury, a moderate risk was estimated for the American robin $(E E Q=13)$. This risk estimate was obtained because the maximum reported 
mercury concentration was used as the exposure point concentration for uptake modeling. That concentration, $6,100 \mu \mathrm{g} / \mathrm{L}$, was a single high value; the next highest mercury concentration was $340 \mu \mathrm{g} / \mathrm{L}$. Using this latter value as the exposure point concentration results in a determination of no risk for the American robin $(\mathrm{EEQ}=0.73)$.

Overall, the EEQ estimates suggest that concentrations of some contaminants in surface water and sediment might pose low risks to aquatic biota, whereas concentrations in surface water pose no risk to terrestrial biota using the springs as drinking water sources. Ingestion of sediment was not considered a significant pathway for contaminant uptake by terrestrial biota.

\subsubsection{Weight of Evidence}

In total, 19 ecological and/or ecotoxicological parameters were evaluated as part of the ecological risk assessment; the results of these evaluations are summarized in Table 6.4. No adverse effects were evident to the invertebrate or vertebrate communities inhabiting Burgermeister Spring and its drainage. The species present in the system are representative of species typically found in similar habitats throughout the Midwest. Although the fish community was limited in diversity and the invertebrate community was classified as slightly impaired (DOE and DA 1997), these conditions are probably the result of the natural, intermittent, and ephemeral nature of the flow within the drainage and the resultant temporal availability of aquatic habitats.

Some toxicity of environmental media was detected for the spring and its drainage. Toxicity of surface water and sediment from Burgermeister Spring proper was detected for the fish Pimephales and the amphipod Hyalella, respectively, as evidenced by reduced survival of test organisms. Surface water and sediment toxicity was also measured at some downstream locations, but no clear toxicity gradient was evident extending downstream from the spring proper. One would expect toxicity to decrease in a downstream direction from the spring as contaminant concentrations become reduced via dilution. However, chronic sediment toxicity to Pimephales was measured only at the farthest downstream location from the spring, the inflow to Lake 34. Similarly, chronic surface water toxicity to the amphibian Xenopus, acute sediment toxicity to Pimephales, and chronic sediment toxicity to Xenopus were detected only at locations downstream of the spring but upstream of the Lake 34 inflow. These results suggest that the source of the observed toxicity is other than Burgermeister Spring. Furthermore, the presence of apparently unaffected invertebrate, fish, and amphibian communities in the drainage at locations where toxicity was detected suggests that although some toxicity may be associated with surface water and sediment in the drainage, local populations have adapted and are tolerant of the contaminant concentrations present in these media.

Contaminant uptake modeling and EEQ estimation indicates no risks to terrestrial biota drinking from the springs. Aquatic biota inhabiting the springs might be susceptible to low to moderate risks from spring water concentrations of iron, manganese, mercury, and uranium and from sediment concentrations of arsenic, lead, and silver. However, as previously discussed, 
TABLE 6.3 Estimated EEQs for Terrestrial Biota Drinking Water from Springs in the Chemical Plant Area and Ordnance Works Area

\begin{tabular}{|c|c|c|c|c|}
\hline \multirow[b]{2}{*}{ Contaminant $\mathrm{t}^{\mathrm{a}}$} & \multicolumn{2}{|c|}{ American Robin } & \multicolumn{2}{|c|}{ White-Tailed Deer } \\
\hline & EEQ & Risk Level $^{\mathrm{b}}$ & EEQ & Risk Level $^{\mathrm{b}}$ \\
\hline \multicolumn{5}{|l|}{ Metals } \\
\hline Antimony & $\mathrm{NBA}^{\mathrm{c}}$ & $N A^{d}$ & 0.01 & No risk \\
\hline Arsenic & 0.02 & No risk & 0.02 & No risk \\
\hline Cadmium & $<0.01$ & No risk & 0.03 & No risk \\
\hline Lead & $<0.01$ & No risk & $<0.01$ & No risk \\
\hline Lithium & NBA & NA & $<0.01$ & No risk \\
\hline Manganese & $<0.01$ & No risk & $<0.01$ & No risk \\
\hline Mercury & 13 & Moderate risk & 0.77 & No risk \\
\hline Molybdenum & $<0.01$ & No risk & $<0.01$ & No risk \\
\hline Selenium & $<0.01$ & No risk & $<0.01$ & No risk \\
\hline Uranium, total & $<0.01$ & No risk & $<0.01$ & No risk \\
\hline Silver & $<0.01$ & No risk & $<0.01$ & No risk \\
\hline \multicolumn{5}{|l|}{ Inorganic anion } \\
\hline Nitrate-N & NBA & NA & $<0.01$ & No risk \\
\hline \multicolumn{5}{|c|}{ Nitroaromatic compounds } \\
\hline $1,3,5-\mathrm{TNB}$ & NBA & NA & $<0.01$ & No risk \\
\hline 1,3-DNB & NBA & NA & $<0.01$ & No risk \\
\hline $2,4,6-\mathrm{TNT}$ & NBA & NA & $<0.01$ & No risk \\
\hline
\end{tabular}

a EEQ values were estimated for all contaminants detected in surface waters from area springs at concentrations above background levels and for which a benchmark value was available.

b EEQ values greater than 1.0 are considered to demonstrate a potential risk to the receptor from a particular contaminant. Values between 1.0 and 10 indicate a low risk, values between 10 and 50 indicate a moderate risk, values between 50 and 100 indicate a high risk, and values greater than 100 indicate extreme risk.

c $\mathrm{NBA}=$ no benchmark available for estimating EEQ.

d $\mathrm{NA}=$ not applicable. 
TABLE 6.4 Summary of the Ecological Effects Assessment of Burgermeister Spring

\begin{tabular}{|c|c|c|c|}
\hline $\begin{array}{c}\text { Organism/ } \\
\text { Assessment Method }\end{array}$ & $\begin{array}{c}\text { Expected Result if Adverse } \\
\text { Effects Present }\end{array}$ & $\begin{array}{l}\text { Observed and Reported } \\
\text { Result }\end{array}$ & $\begin{array}{c}\text { Adverse } \\
\text { Effect }\end{array}$ \\
\hline $\begin{array}{l}\text { Aquatic invertebrate } \\
\text { surveys }\end{array}$ & $\begin{array}{l}\text { Low abundance and species diversity; } \\
\text { community dominated by only a few } \\
\text { taxa }\end{array}$ & $\begin{array}{l}\text { Slightly impaired invertebrate } \\
\text { community typical of ephemeral, } \\
\text { intermittent habitats }\end{array}$ & No \\
\hline Fish surveys & $\begin{array}{l}\text { Low abundance; adverse external } \\
\text { conditions, such as lesions or tumors, } \\
\text { suggestive of contaminant exposure }\end{array}$ & $\begin{array}{l}\text { No fish collected from the spring } \\
\text { proper, and none expected due to } \\
\text { blocked access from downstream } \\
\text { habitats; downstream community } \\
\text { comprised of species typical of similar } \\
\text { habitats in the Midwest; no evidence } \\
\text { of adverse external conditions }\end{array}$ & No \\
\hline Amphibian surveys & $\begin{array}{l}\text { Low abundance; adverse external } \\
\text { conditions, such as lesions or tumors, } \\
\text { suggestive of contaminant exposure }\end{array}$ & $\begin{array}{l}\text { Six species collected from spring area, } \\
\text { comparable to community from } \\
\text { reference location; species typical of } \\
\text { similar habitats in the Midwest; no } \\
\text { evidence of adverse external } \\
\text { conditions }\end{array}$ & No \\
\hline $\begin{array}{l}\text { Daphnia, surface water, } \\
\text { 96-hour acute toxicity }\end{array}$ & Reduced survival & No reduction in survival & No \\
\hline $\begin{array}{l}\text { Hyalella, surface water, } \\
\text { 96-hour acute toxicity }\end{array}$ & Reduced survival & No reduction in survival & No \\
\hline $\begin{array}{l}\text { Pimephales, surface water, } \\
96 \text {-hour acute toxicity }\end{array}$ & Reduced survival & $\begin{array}{l}62.5 \% \text { reduction in survival at the } \\
\text { spring and nearest downstream } \\
\text { sampling location }\end{array}$ & Yes \\
\hline $\begin{array}{l}\text { Xenopus, surface water, } \\
\text { 96-hour acute toxicity }\end{array}$ & Reduced survival & No reduction in survival & No \\
\hline $\begin{array}{l}\text { Daphnia, surface water, } \\
\text { 7-day chronic toxicity }\end{array}$ & Reduced survival & No reduction in survival & No \\
\hline $\begin{array}{l}\text { Hyalella, surface water, } \\
7 \text {-day chronic toxicity }\end{array}$ & Reduced survival & No reduction in survival & No \\
\hline $\begin{array}{l}\text { Pimephales, surface water, } \\
7 \text {-day chronic toxicity }\end{array}$ & Reduced survival and growth & No reduction in survival or growth & No \\
\hline $\begin{array}{l}\text { Xenopus, surface water, } \\
\text { 7-day chronic toxicity }\end{array}$ & Reduced survival and growth & $\begin{array}{l}30 \% \text { reduction in survival at one } \\
\text { location downstream of the spring; no } \\
\text { reduction in survival at other } \\
\text { locations; no reduction in growth }\end{array}$ & Yes \\
\hline $\begin{array}{l}\text { Daphnia, sediment, } \\
\text { 96-hour acute toxicity }\end{array}$ & Reduced survival & No reduction in survival & No \\
\hline
\end{tabular}


TABLE 6.4 (Cont.)

\begin{tabular}{|c|c|c|c|}
\hline $\begin{array}{c}\text { Organism/ } \\
\text { Assessment Method }\end{array}$ & $\begin{array}{c}\text { Expected Result if Adverse } \\
\text { Effects Present }\end{array}$ & $\begin{array}{c}\text { Observed and Reported } \\
\text { Result } \\
\end{array}$ & $\begin{array}{l}\text { Adverse } \\
\text { Effect }\end{array}$ \\
\hline $\begin{array}{l}\text { Hyalella, sediment, } \\
96 \text {-hour acute toxicity }\end{array}$ & Reduced survival & No reduction in survival & No \\
\hline $\begin{array}{l}\text { Pimephales, sediment, } \\
\text { 96-hour acute toxicity }\end{array}$ & Reduced survival & $\begin{array}{l}25 \% \text { reduction in survival at the first } \\
\text { downstream sampling location below } \\
\text { the spring }\end{array}$ & Yes \\
\hline $\begin{array}{l}\text { Xenopus, sediment, } \\
\text { 96-hour acute toxicity }\end{array}$ & Reduced survival & No reduction in survival & No \\
\hline $\begin{array}{l}\text { Daphnia, sediment, } \\
\text { 7-day chronic toxicity }\end{array}$ & Reduced survival & No reduction in survival & No \\
\hline $\begin{array}{l}\text { Hyalella, sediment, } \\
\text { 7-day chronic toxicity }\end{array}$ & Reduced survival & $\begin{array}{l}18 \% \text { reduction in survival at the } \\
\text { spring; no effects at downstream } \\
\text { locations }\end{array}$ & Yes \\
\hline $\begin{array}{l}\text { Pimephales, sediment, } \\
\text { 7-day chronic toxicity }\end{array}$ & Reduced survival and growth & $\begin{array}{l}50 \% \text { reduction in survival at farthest } \\
\text { downstream sampling location }\end{array}$ & Yes \\
\hline $\begin{array}{l}\text { Xenopus, sediment, } \\
\text { 7-day chronic toxicity }\end{array}$ & Reduced survival and growth & $\begin{array}{l}27 \% \text { reduction in survival at first } \\
\text { sampling location downstream of the } \\
\text { spring; no reduction in survival at } \\
\text { other locations; no reduction in growth }\end{array}$ & Yes \\
\hline
\end{tabular}

Burgermeister Spring and waters downstream support invertebrate, fish, and amphibian communities typical of similar habitats elsewhere in the Midwest and do not appear to be adversely affected by contaminant concentrations at this time. Because of physical conditions independent of any contamination (such as low flow), other springs in the area are not expected to support extensive aquatic habitats or biota, and risks to these resources from current contaminant levels are expected to be very minor or nonexistent.

\subsubsection{Ecological Significance}

For most of the contaminants detected in the surface water and sediment from springs, little or no potential is indicated for significant adverse ecological effects to aquatic or terrestrial biota. Because of the small and temporal nature of most of the springs, relatively few biota are anticipated to be exposed to contaminants at these habitats. The most likely exposed biota (and thus those potentially at greatest risk) at the springs (excluding Burgermeister Spring) are aquatic invertebrates. However, the abundance and diversity of biota in the springs is limited by the physical nature of 
these habitats and is independent of contaminants. Thus, the magnitude and nature of potential impacts at these springs would be very small and would have little ecological significance to the aquatic invertebrate populations in the area. Furthermore, these springs represent a very small fraction of the total aquatic habitat available in the August A. Busch Memorial Conservation Area.

The receptors most likely at risk at Burgermeister Spring are fish and aquatic invertebrates. Although some sediment and surface water toxicity is indicated for Burgermeister Spring, the ecological significance of this toxicity is very small and should not be expected to adversely affect aquatic resources of the area. The results of the biotic surveys and toxicity tests indicate that the aquatic community in Burgermeister Spring is typical of similar habitats throughout the Midwest and shows no evidence of being adversely affected by contaminants in surface water and sediment.

\subsection{UNCERTAINTIES RELATED TO RISK CHARACTERIZATION}

A number of uncertainties are inherent in estimating the ADD and EEQ, and these uncertainties could affect both the estimated values of these end points and the final risk characterization.

The principal uncertainties associated with the model assumptions are related to (1) estimation of contaminant uptake and assimilation and (2) use of a constant ingestion rate over the entire home range of a species. The uptake and assimilation of contaminants by the receptor species is affected by a variety of factors not addressed by the uptake models. These factors include, but are not limited to, contaminant solubility in biological fluids, species metabolism, contaminant biotransformation, and depuration. For some biota, it is unlikely that the uptake and assimilation of a contaminant is $100 \%$ efficient; for other biota, efficiency may approach $100 \%$. Thus, the $100 \%$ uptake and assimilation assumption used in the uptake modeling likely overestimates the true degree of contaminant assimilation by the receptor species.

The assumption that the drinking water ingestion rate is constant over the entire home range is probably inaccurate, particularly for species with large home ranges, such as the white-tailed deer. Most resources in the environment, including water, are not distributed homogeneously but rather in a patchy, heterogeneous manner. As a consequence, drinking would also occur in a patchy manner. However, this assumption is conservative and should not affect the overall ADD estimate.

An additional uncertainty related to the risk characterization is associated with the unavailability of suitable benchmark values for some contaminants and terrestrial receptors. For example, no avian benchmark values were found for nitroaromatic compounds. Although it was possible to model uptake of nitroaromatic compounds by the American robin, it was not possible to estimate risks because of the absence of suitable benchmark values. However, unacceptable risks are not anticipated from the COECs for which benchmark values are not available. For the terrestrial receptors, no risks were identified for those COECs for which benchmark values were available. Even using the maximum reported contaminant concentrations, the estimated risks were very low (typically $<0.01$ ). The estimated doses for the COECs with no benchmark values were similarly 
very low, and thus no risks (i.e., very low estimated risk of $<0.01$ ) would be expected for these contaminants.

\subsection{SUMMARY}

The results of biotic surveys, media toxicity testing, and contaminant uptake modeling indicate that current contaminant levels in surface water and sediment in springs pose little or no risk to the aquatic and terrestrial biota of the area. Although some surface water and sediment toxicity was detected in Burgermeister Spring, and the concentrations of some contaminants exceed ambient water quality criteria for the protection of freshwater aquatic biota, there is no evidence that aquatic biota inhabiting the spring and downstream habitats are being impacted. Uptake modeling indicates no risk to terrestrial biota that use area springs for drinking water. These results show that contaminant concentrations in surface water and sediment at these springs pose little or no risks to ecological resources of the area, and remediation from an ecological perspective is not warranted at this time. 


\section{SUMMARY AND CONCLUSION}

A combined assessment addressing human health and ecological impacts was performed to evaluate conditions at the GWOUs. The human health component of this BRA included an evaluation of the radiological and chemical risks from contamination in the 15 springs and in the shallow aquifer system that is common to both the chemical plant area and ordnance works area. Recent data obtained from the joint DOE/DA sampling rounds of May and August 1995 were used to calculate potential human health impacts. The ecological risk assessment evaluated potential risks to aquatic and terrestrial biota from exposure to contaminants in surface water at the springs. The assessment also focused on laboratory and field studies of Burgermeister Spring because the aquatic habitats associated with this spring are more permanent than the habitats at other springs in the area and thus may be used by a greater variety and number of biota than habitats at other springs.

\subsection{HUMAN HEALTH RISK ASSESSMENT}

\subsubsection{Methodology}

The human health risk assessment was conducted in accordance with the procedure recommended by the EPA (1989b). The procedure involves the following four steps: (1) COPC identification, (2) exposure assessment, (3) toxicity assessment, and (4) risk characterization.

Chemical COPCs were identified as those determined to be greater than background as discussed in the RI. The groundwater COPCs identified were lithium, molybdenum, uranium, chloride, nitrates, sulfates, nitroaromatic compounds, TCE, and 1,2-DCE. Uranium has also been identified as the only radioactive COPC. The spring water COPCs identified were antimony, cadmium, iron, lithium, manganese, mercury, molybdenum, silver, uranium, and nitroaromatic compounds.

Current and future land-use projections were incorporated into identifying the potential human receptor as part of the exposure assessment. A recreational scenario was considered to be appropriate on the basis of current and projected future land use at the chemical plant area and the ordnance works area. Exposure of Army reservists that visit the training area was not evaluated separately because there are no active springs within the boundaries of the training area. Also, the estiamated risks calculated for the recreational visitor are representative of those for the training troops because the exposure parameters (e.g., duration and frequency) would be similar. Although potential risk to the recreational receptor would likely provide information representative of future conditions at both areas with regard to springs, calculations were also carried out for a hypothetical future resident to provide reasonable upper-bound information regarding potential risk from groundwater contamination. 
To determine potential exposure of a recreational visitor, a hazard index and the chemical and radiological carcinogenic risk were calculated for each of the 15 springs evaluated, using the maximum value from the 1995 joint DOE/DA sampling rounds for each COPC in spring water. Similar calculations were performed for each of 155 wells to determine potential exposure of a hypothetical future resident to groundwater contamination. The primary pathway of concern in both cases was ingestion. Standard EPA-recommended exposure parameters were used in the calculations (EPA 1995b). Current contaminant concentrations were also assumed for future scenarios. This approach is considered conservative; contaminant concentrations are expected to decrease with time as a result of source removals currently ongoing at both the chemical plant area and the ordnance works area.

\subsubsection{Results}

Neither carcinogenic risk nor noncarcinogenic health effects are indicated for the recreational visitor incidentally ingesting spring water at the 15 springs evaluated; these results are expected to be representative of all springs located in the area covered by the GWOUs. The radiological risk estimates range from $4 \times 10^{-9}$ to $2 \times 10^{-6}$. These values are low and well within the acceptable risk range of $1 \times 10^{-6}$ to $1 \times 10^{-4}$ recommended by the EPA (1989b). The chemical risk estimates are similarly low, ranging from $2 \times 10^{-10}$ to $3 \times 10^{-7}$. The EPA has provided a quantitative measure for adverse health effects other than cancer: a hazard index greater than 1 indicates potential adverse health effects. The hazard indices estimated for the recreational visitor at the springs range from $<0.001$ to 0.2 .

The well-by-well calculations for the hypothetical future resident scenario indicate that, excluding TCE contributions at the 155 wells evaluated, chemical risk estimates for four wells are slightly higher than $1 \times 10^{-4}$. The chemical risk estimates for these wells range from $1 \times 10^{-7}$ to $2 \times 10^{-4}$. The upper end of this range is attributable to nitroaromatic compounds detected at well MWV-09, located north of the groundwater divide. The radiological risk estimates range from $7 \times 10^{-8}$ to $7 \times 10^{-5}$, all within the acceptable risk range. With the inclusion of risk from TCE, risk estimates at three additional wells exceed $1 \times 10^{-4}: 1 \times 10^{-3}$ at MW-2038, $4 \times 10^{-4}$ at MW-2037, and $3 \times 10^{-4}$ at MWS-21. These wells are weathered wells near the raffinate pits.

The hazard indices for 43 of the 155 wells evaluated are greater than 1 . Of the 43 , hazard indices for 27 wells are attributable to nitroaromatic compounds. Elevated nitrates occur mostly in the chemical plant area 2000- and 3000-series wells; 15 hazard indices that are greater than 1 are attributable to nitrate concentrations in these wells. The estimated hazard index for well MW-4024 is 1 ; uranium concentrations in this well contributed to 0.84 of this hazard index of 1 . 


\subsubsection{Summary}

The radiological and chemical risk assessments have been presented separately because the methodologies for estimating the carcinogenic risks from exposures to radionuclides and chemicals differ considerably. However, the total carcinogenic risk to an individual is the result of exposure to both radiological and chemical risks, assuming that the carcinogenic effects are neither antagonistic nor synergistic. Summing the radiological and chemical carcinogenic risks for the recreational visitor (considered representative of current and expected future land use) would result in risk levels still below or at the lower end of the acceptable risk range. Similarly, summing the radiological and chemical carcinogenic risks to the hypothetical future resident would not result in a large increase in the overall results because the majority of the radiological risk results are well within the acceptable risk range. Overall, the more significant contributors to potential human health risk from the groundwater pathways are TCE, nitrates, and nitroaromatic compounds.

\subsection{ECOLOGICAL RISK ASSESSMENT}

\subsubsection{Methodology}

The ecological risk assessment for the GWOUs employed a number of approaches for evaluating risks to ecological resources that use springs on the chemical plant area and ordnance works area. Risks to aquatic biota were evaluated by using biotic surveys and media toxicity testing and by comparing media concentrations to ecological benchmark ("safe") media concentrations. Risks to terrestrial biota were evaluated by modeling contaminant uptake and comparing the predicted doses to species-specific benchmark doses. Contaminant data used in the assessment included the same surface water data used in the human health risk assessment, as well as sediment data collected specifically for the ecological risk assessment at Burgermeister Spring and selected downstream locations.

Biotic surveys for aquatic invertebrates, fish, and amphibians were conducted at Burgermeister Spring and its downstream drainage. The data collected from these surveys allowed for a determination of the status of the biotic communities currently exposed to contaminants in surface water and sediment at the spring. Macroinvertebrates and fish samples were collected from Burgermeister Spring and its downstream locations, and tissue analyses were conducted to evaluate contaminant bioconcentration by aquatic biota. Toxicity testing of surface water and sediment from the spring and downstream locations included acute and chronic toxicity testing of aquatic invertebrates, fish, and amphibians. These tests determined whether current contaminant concentrations in the surface water and sediment are toxic to aquatic biota. Contaminant uptake from the ingestion of surface water was modeled for two terrestrial receptor species, the white-tailed deer and the American robin. The uptake modeling employed species-specific exposure factors, and the exposure point concentrations were the maximum reported contaminant concentrations in surface water from springs in the chemical plant area and ordnance works area. 


\subsubsection{Results}

The survey results for macroinvertebrates, fish, and amphibians that inhabit the Burgermeister Spring drainage indicated no evidence of adverse effects to these aquatic biota. The spring was determined to contain generally good aquatic habitat, and the species present are typical of those found in similar habitats throughout the Midwest. Although the fish community was limited in diversity and the macroinvertebrate community was categorized as slightly impaired, the communities are likely affected by the physical nature of the spring and its drainage rather than contaminant levels. Flow in the uppermost portion of Burgermeister Spring is maintained by groundwater discharge at the spring. Under low-flow conditions, as commonly occur in the summer, the stream drainage below the spring becomes intermittent and portions of the habitat become dry. Surveys of the amphibian community identified a community typical of similar habitats in the Midwest.

The results of toxicity testing indicate a potential for some toxicity to fish and invertebrates from surface water and sediment in Burgermeister Spring proper. Surface water and sediment toxicity was also measured at some locations downstream of the spring, but no clear toxicity gradient was evident extending downstream. However, the presence of apparently unaffected macroinvertebrate, fish, and amphibian communities in the drainage at locations where media toxicity was detected suggests that local populations are tolerant of (or have adapted to) the contaminant levels present in surface water and sediment in the Burgermeister Spring drainage. Tissue analyses revealed relatively low levels of contaminant bioconcentration, all below levels of concern.

Modeling results for contaminant uptake by the white-tailed deer and the American robin drinking from Burgermeister Spring (but using maximum contaminant concentrations reported from all springs) predict very low levels of contaminant uptake by these species. Risk estimates for terrestrial biota based on the modeled contaminant doses indicate no risks to terrestrial biota drinking from Burgermeister Spring or other springs in the area.

Risk estimates for aquatic biota based on media concentrations indicate that spring water concentrations of iron, manganese, mercury, and uranium and sediment concentrations of arsenic, lead, and silver might pose low to moderate risks to aquatic biota. However, the aquatic community in Burgermeister Spring is typical of similar habitats elsewhere in the Midwest and does not appear to be adversely affected by contaminant concentrations at this time. Few of the other springs in the area provide suitable habitat and, at best, naturally support only very limited aquatic communities.

\subsubsection{Summary}

On the basis of the results of biotic surveys, media toxicity testing, tissue analyses, mediabased risk calculations, and contaminant uptake modeling, current contaminant levels in surface water and sediment in area springs pose little or no risk to aquatic or terrestrial biota of the Weldon Spring area. Risk calculations indicated a potential for low to moderate risks to aquatic biota from 
some contaminants in springs, and surface water and sediment toxicity was detected for Burgermeister Spring. However, biotic surveys of Burgermeister Spring and downstream habitats found no evidence that aquatic biota inhabiting this spring are being adversely impacted, and few other springs naturally provide sufficient permanent habitat to support more than only very limited aquatic communities. Uptake modeling indicates no risks to terrestrial wildlife using the area springs for drinking water.

\subsection{CONCLUSION}

Carcinogenic (radiological and chemical) risk and noncarcinogenic health effects are not indicated for the recreational visitor at the chemical plant area and the ordnance works area. The recreational visitor potentially exposed to spring water is considered to be representative of current and future land uses at both areas. Potential incremental carcinogenic risk and noncarcinogenic health effects to an Army reservist training at the ordnance works area are also not indicated. The results of the risk assessment for springs presented here are consistent with those in previous risk assessments.

Risk calculations for groundwater ingestion by a hypothetical future resident indicate that high concentrations of nitrates and nitroaromatic compounds in several wells used for monitoring known source areas contribute to high (greater than 1) hazard indices. Several wells in the vicinity of the raffinate pits and sludge in the pits have been determined to contain high concentrations of nitrates. Several wells in both the chemical plant and ordnance works areas also contain amounts of nitroaromatic compounds that could potentially contribute to carcinogenic risks slightly over the upper end of the risk range. The use of the second (lower) data point from the joint DOE/DA sampling rounds would have resulted in lower risk estimates that fall within the acceptable risk range. Radiological risks from uranium are within the acceptable risk range. Monitoring wells and springs with the highest estimated risks and hazard indices are depicted in Figure 7.1.

Additionally, in interpreting the results for groundwater, one should consider that if a future resident did draw groundwater as a household drinking water supply, the COPCs, if present, would be in more dilute concentrations than those used for the calculations in this assessment. In addition, future concentrations for both groundwater and spring water contaminants would most likely be lower because active removal of contaminant sources is currently ongoing and concentrations in groundwater are expected to decrease with time. To provide another perspective, the hazard indices and carcinogenic risks from groundwater use would be two orders of magnitude lower for the hypothetical recreational user than would be expected for the hypothetical residential user.

Finally, the risk estimates indicate that of the COPCs evaluated, nitrates and nitroaromatic compounds may be of concern due to their contributions to relatively high hazard indices. These results also indicate that contaminant concentrations tend to be higher in the weathered unit rather than in the unweathered unit of the aquifer of concern, as evidenced by generally higher risk estimates for the wells completed in the weathered unit. 


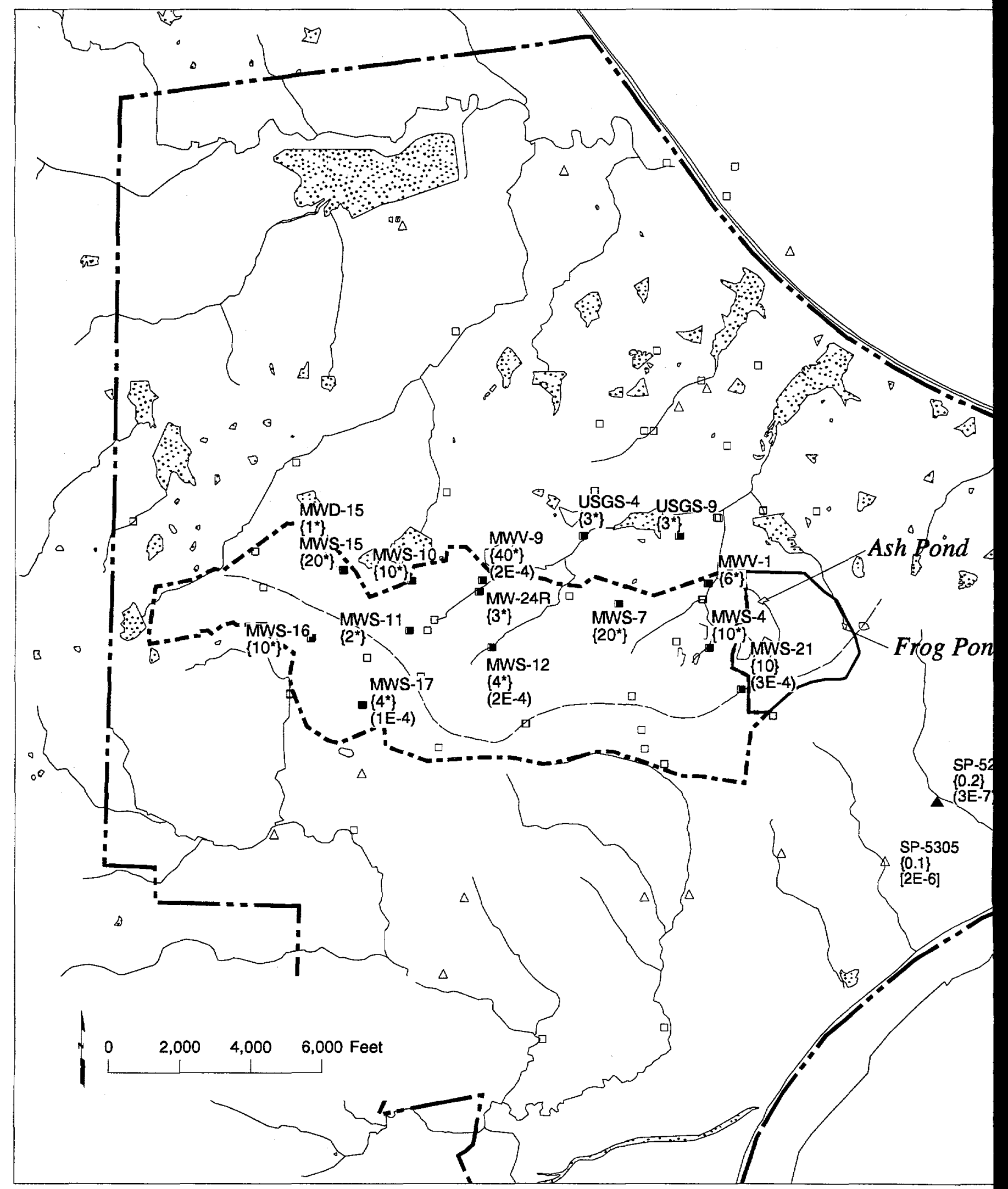

FIGURE 7.1 Monitoring Wells and Springs with the Highest Estimated Risks and Hazard Indices 


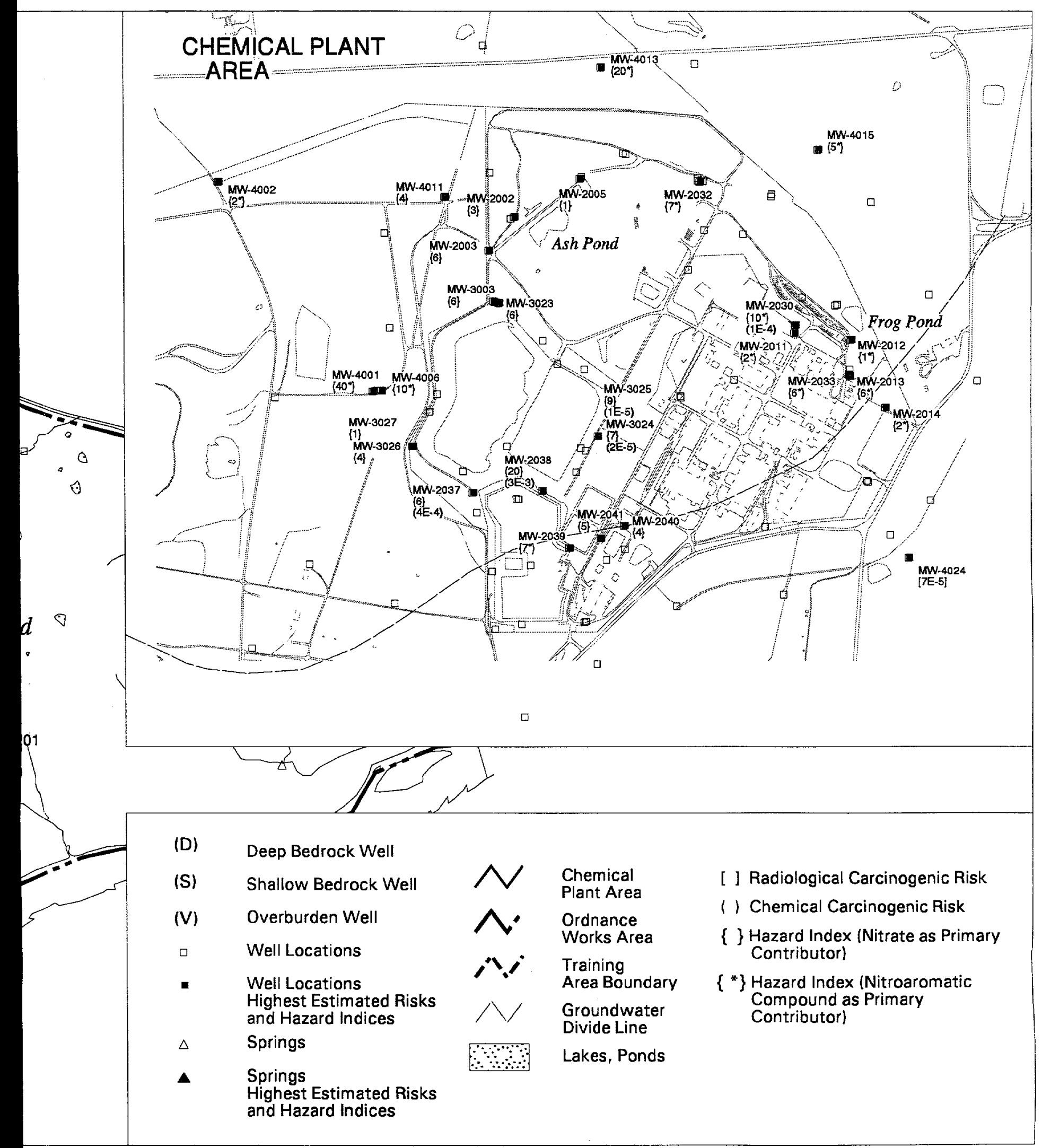




\section{REFERENCES}

Agency for Toxic Substances and Disease Registry, 1990, Toxicological Profile for Silver, TP-90-24, U.S. Public Health Service, Atlanta, Ga.

Baysinger-Daniel, C., 1996, memorandum from C. Baysinger-Daniel (Missouri Department of Health, Jefferson City, Mo.) to M. Picel (Argonne National Laboratory, Argonne, Ill.), with attachment, Aug. 26.

Brodsky, A., 1996, Review of Radiation Risks and Uranium Toxicity with Application to Decisions Associated with Decommissioning Clean-up Criteria, RSA Publications, Hebron, Conn.

Chien, L., et al., 1968, "Infantile Gastroenteritis due to Water with High Sulfate Content," Canadian Medical Association Journal 99:102-104.

Clench, M.H., and R.C. Leberman, 1978, "Weights of 151 Species of Pennsylvania Birds Analyzed by Month, Age, and Sex," Bulletin of Carnegie Museum of Natural History. [As cited in EPA (1993).]

Crigler, D., 1992, personnel communication from D. Crigler (Wildlife Management Biologist, August A. Busch Memorial Wildlife Area, St. Charles, Mo.) to G. Valett (Project Management Contractor, Weldon Spring Site Remedial Action Project, St. Charles, Mo.), as documented in memorandum from G. Valett to R. Ferguson (Jacobs Engineering Group, Inc., Weldon Spring Site Remedial Action Project, St. Charles, Mo.), May 21.

DOE: see U.S. Department of Energy.

Eisler, R., 1985, Selenium Hazards to Fish, Wildlife, and Invertebrates: a Synoptic Review, Contaminant Hazard Reviews Report No. 5, Biological Report 85(1.5), U.S. Fish and Wildlife Service, Patuxent Wildlife Research Center, Laurel, Md.

Eisler, R., 1987, Mercury Hazards to Fish, Wildlife, and Invertebrates: a Synoptic Review, Contaminant Hazard Reviews Report No. 19, Biological Report 85(1.10), U.S. Fish and Wildlife Service, Patuxent Wildlife Research Center, Laurel, Md.

EPA: see U.S. Environmental Protection Agency.

Hull, R.N., and G.W. Suter, 1994, Toxicological Benchmarks for Screening Contaminants of Potential Concern for Effects on Sediment Associated Biota: 1994 Revision, ES/ER/TM-95/R1, prepared by Oak Ridge National Laboratory, Environmental Sciences Division, Oak Ridge, Tenn., for the U.S. Department of Energy, June. 
IAEA: see International Atomic Energy Agency.

International Atomic Energy Agency, 1992, Effects of Ionizing Radiation on Plants and Animals at Levels Implied by Current Radiation Protection Standards, Technical Report Series No. 332, Vienna, Austria.

International Technology Corporation, 1992, Final Remedial Investigation Report, Weldon Spring Ordnance Works, Weldon Spring, Missouri, Project No. 312071, prepared by IT Corporation, Itasca, Ill., for U.S. Department of the Army, Corps of Engineers, Kansas City District, Nov.

International Technology Corporation, 1993a, Final Remedial Investigation Report, Weldon Spring Training Area, Weldon Spring, Missouri, 2 vol., Project No. 312188, prepared by IT Corporation, Itasca, Ill., for U.S. Department of the Army, Corps of Engineers, Kansas City District, March.

International Technology Corporation, 1993b, Revised Final Baseline Risk Assessment, Weldon Spring Ordnance Works, Weldon Spring, Missouri, Project No. 312071, prepared by IT Corporation, Itasca, Ill., for U.S. Department of the Army, Corps of Engineers, Kansas City District, May (updated Oct.).

International Technology Corporation, 1993c, Groundwater Monitoring Report, First Yearly Sampling Round, Weldon Spring Ordnance Works, Project No. 312071, prepared by IT Corporation, Itasca, Ill., for U.S. Department of the Army, Corps of Engineers, Kansas City District, April.

International Technology Corporation, 1993d, 1993: Second Quarterly Groundwater Monitoring Report, Weldon Spring Ordnance Works, Project No. 312071, prepared by IT Corporation, Itasca, IIl., for U.S. Department of the Army, Corps of Engineers, Kansas City District, July.

International Technology Corporation, 1993e, 1993: Third Quarterly Groundwater Monitoring Report, Weldon Spring Ordnance Works, Project No. 312071, prepared by IT Corporation, Itasca, IIl., for U.S. Department of the Army, Corps of Engineers, Kansas City District, Sept.

International Technology Corporation, 1993f, Final Feasibility Study, Former Weldon Spring Ordnance Works, Weldon Spring, Missouri, Project No. 312263, prepared by IT Corporation, Itasca, III., for U.S. Department of the Army, Corps of Engineers, Kansas City District, July.

International Technology Corporation, 1994a, 1993: Fourth Quarterly Groundwater Monitoring Report, Weldon Spring Ordnance Works, Project No. 312071, prepared by IT Corporation, Itasca, Ill., for U.S. Department of the Army, Corps of Engineers, Kansas City District, Jan.

International Technology Corporation, 1994b, 1994: Second Yearly Groundwater Monitoring Report, Weldon Spring Ordnance Works, Project No. 312071, prepared by IT Corporation, Itasca, Ill., for U.S. Department of the Army, Corps of Engineers, Kansas City District, April. 
International Technology Corporation, 1994c, 1994: Second Quarterly Groundwater Monitoring Report, Weldon Spring Ordnance Works, Project No. 312430, prepared by IT Corporation, Itasca, Ill., for U.S. Department of the Army, Corps of Engineers, Kansas City District, Aug.

International Technology Corporation, 1994d, 1994: Third Quarterly Groundwater Monitoring Report, Weldon Spring Ordnance Works, Project No. 312430, prepared by IT Corporation, Kansas City, Mo., for U.S. Department of the Army, Corps of Engineers, Kansas City District, Oct.

International Technology Corporation, 1995a, 1994: Fourth Quarterly Groundwater Monitoring Report, Weldon Spring Ordnance Works, Project No. 312430, prepared by IT Corporation, Kansas City, Mo., for U.S. Department of the Army, Corps of Engineers, Kansas City District, Jan.

International Technology Corporation, 1995b, 1995: Third Yearly Groundwater Monitoring Report, Weldon Spring Ordnance Works, Project No. 312071, prepared by IT Corporation, Kansas City, Mo., for U.S. Department of the Army, Corps of Engineers, Kansas City District, April.

IT Corporation: see International Technology Corporation.

Jensen, L.S., R.P. Peterson, and L. Falen, 1974, "Inducement of Enlarged Hearts and Muscular Dystrophy in Turkey Poults with Dietary Silver," Poultry Science 53:57-64.

Kleeschulte, M.J., 1991, Geohydrology of Bedrock Aquifers and Public Supply and Domestic Water Use, 1962-85, in St. Charles County, Missouri, proceedings of the Geosciences Workshop, February 21, O'Fallon, Mo., Weldon Spring Site Remedial Action Project, U.S. Department of Energy.

Long, E.R., and L.G. Morgan, 1990, Potential for Biological Effects of Sediment-Sorbed Contaminants Tested in the National Status and Trends Program, NOAA/TM/NOS/OMA-52, National Ocean Service, Office of Oceanography and Marine Assessment, Rockville, Md.

Maynard, E.A., and H.C. Hodge, 1949, "Studies of the Toxicity of Various Uranium Compounds When Fed to Experimental Animals," pp. 309-376 in The Pharmacology and Toxicology of Uranium Compounds, Vol. I, C. Voegtlin and H.C. Hodge (editors), McGraw-Hill Book Company, New York, N.Y.

Missouri Department of Natural Resources, 1992, Rules of Department of Natural Resources: Division 20 - Clean Water Commission; Chapter 7 - Water Quality, Code of State Regulations 10 CSR 20-7.031 (Water Quality Standards), Jefferson City, Mo.

National Council on Radiation Protection and Measurements, 1991, Effects of Ionizing Radiation on Aquatic Organisms, NCRP Report No. 109, Bethesda, Md. 
National Research Council, 1977, Drinking Water and Health, National Academy of Sciences, Washington, D.C.

National Research Council, 1989, Recommended Dietary Allowances, 10th edition, National Academy Press, Washington, D.C.

NCRP: see National Council on Radiation Protection and Measurements.

Poston, T.M., et al., 1984, "Toxicity of Uranium to Daphnia magna," Water, Air, and Soil Pollution 22:289-298.

Rose, K.S.B., 1992, "Lower Limits of Radiosensitivity in Organisms, Excluding Man," Journal of Environmental Radioactivity 15:113-133.

Sample, B.E., et al., 1996, Toxicological Benchmarks for Wildlife: 1996 Revision, ES/ER/TM-86/ R2, prepared by Oak Ridge National Laboratory, Oak Ridge, Tenn., for U.S. Department of Energy, Washington, D.C., June.

Schwartz, C.W., and E.R. Schwartz, 1981, The Wild Mammals of Missouri, University of Missouri Press and Missouri Department of Conservation, Columbia, Mo.

Suter, G.W., and C.L. Tsao, 1996, Toxicological Benchmarks for Screening Potential Contaminants of Concern for Effects on Aquatic Biota: 1996 Revision, ES/ER/TM-96/R2, prepared by Oak Ridge National Laboratory, Environmental Sciences Division, Oak Ridge, Tenn., for U.S. Department of Energy, June.

Talmage, S.S., and D.M. Opresko, 1996, Ecological Criteria/Toxicological Benchmarks for Screening Effects of Munitions Compounds on the Environment, draft, prepared by Oak Ridge National Laboratory, Oak Ridge, Tenn., for U.S. Environmental Protection Agency, Cincinnati, Ohio, April.

U.S. Department of Energy, 1992, Baseline Assessment for the Chemical Plant Area of the Weldon Spring Site, DOE/OR/21548-091, prepared by Argonne National Laboratory, Argonne, Ill., for U.S. Department of Energy, Weldon Spring Remedial Action Project, St. Charles, Mo., Nov.

U.S. Department of Energy, 1993, Baseline Risk Assessment for Exposure to Contaminants at the St. Louis Site, St. Louis, Missouri, DOE/OR/23701-41.1, prepared by Argonne National Laboratory, Argonne, Ill., for U.S. Department of Energy, Oak Ridge Operations Office, Oak Ridge, Tenn., Nov.

U.S. Department of Energy, 1995, Work Plan for the Remedial Investigation/Feasibility Study for the Groundwater Operable Units at the Chemical Plant Area and the Ordnance Works Area, Weldon Spring, Missouri, DOE/OR/21548-567, prepared by Argonne National Laboratory, Argonne, Ill., 
for U.S. Department of Energy, Weldon Spring Site Remedial Action Project, Weldon Spring, Mo., Aug.

U.S. Department of Energy, 1996, Engineering Evaluation/Cost Analysis for the Proposed Removal Action at the Southeast Drainage near the Weldon Spring Site, Weldon Spring, Missouri, DOE/OR/21548-584, prepared by Argonne National Laboratory, Argonne, Ill, for U.S. Department of Energy, Weldon Spring Site Remedial Action Project, Weldon Spring, Mo., Aug.

U.S. Department of Energy and U.S. Department of the Army, 1997, Remedial Investigation for the Groundwater Operable Units at the Chemical Plant Area and the Ordnance Works Area, Weldon Spring Site, Weldon Spring, Missouri, DOE/OR/21548-571, Draft Final, prepared by MK-Ferguson Company and Jacobs Engineering Group, Inc., Weldon Spring, Mo., and Argonne National Laboratory, Argonne, Ill., for U.S. Department of Energy Oak Ridge Operations Office, Oak Ridge, Tenn., and U.S. Department of the Army, Corps of Engineers, Kansas City District, Mo., Feb.

U.S. Environmental Protection Agency, 1986, Quality Criteria for Water, EPA/440/5-86-001, Office of Water Regulations and Standards, Washington, D.C., May.

U.S. Environmental Protection Agency, 1989a, Rapid Bioassessment Protocols for Use in Streams and Rivers: Benthic Macroinvertebrates and Fish, EPA/444/4-89-001, Assessment and Watershed Protection Division, Office of Water, Washington, D.C., May.

U.S. Environmental Protection Agency, 1989b, Risk Assessment Guidance for Superfund, Volume 1, Human Health Evaluation Manual (Part A), EPA/540/1-89/002, U.S. Environmental Protection Agency, Office of Emergency and Remedial Response, Washington, D.C.

U.S. Environmental Protection Agency, 1989c, Risk Assessment Guidance for Superfund, Volume II, Environmental Evaluation Manual, Interim Final, EPA/540/1-89/001, Office of Emergency and Remedial Response, Washington, D.C., March.

U.S. Environmental Protection Agency, 1992a, Dermal Exposure Assessment: Principles and Applications, EPA/600/8-91-011B, Interim Report, Office of Research and Development, Washington, D.C., Jan.

U.S. Environmental Protection Agency, 1992b, Framework for Ecological Risk Assessment, EPA/630/R-92/001, Risk Assessment Forum, Washington, D.C., Feb.

U.S. Environmental Protection Agency, 1993, Wildlife Exposure Factors Handbook, Vol. 1, EPA/600/R-93/187a, Office of Research and Development, Washington, D.C., Dec.

U.S. Environmental Protection Agency, 1995a, EPA Region III Screening Levels, Region III, Philadelphia, Pa. 
U.S. Environmental Protection Agency, 1995b, Exposure Factors Handbook, EPA/600/P-95/002A (PB95-252532), Office of Research and Development, Washington, D.C., June.

U.S. Environmental Protection Agency, 1995c, Health Effects Assessment Summary Tables, FY-1995 Annual, EPA/540/R-95-036, Office of Solid Waste and Emergency Response, May.

U.S. Environmental Protection Agency, 1996a, Ecotox Thresholds, ECO Update, Publication 9345.0-12FSI, EPA 540/F-95/038 (PB95-963324), Office of Solid Waste and Emergency Response, Washington, D.C., Jan.

U.S. Environmental Protection Agency, 1996b, "Risk Assessment Issue Paper for: Carcinogenicity Information for Trichloroethylene (TCE) (CASRN 79-01-6)," Attachment III to facsimile transmittal to H. Hartmann (Argonne National Laboratory, Argonne, Ill.) from Superfund Technical Support Center, Cincinnati, Ohio, Oct. 17.

U.S. Environmental Protection Agency, 1996c, Drinking Water Regulations and Health Advisories, EPA 882-B-96-002, Office of Water, Washington, D.C., Oct., pp. 1-11.

U.S. Environmental Protection Agency, 1997, Integrated Risk Information System, database, accessed February 1997.

Venugopal, B., and T.D. Luckey, 1978, Metal Toxicity in Mammals, Vol. 2, Plenum Press, New York, N.Y.

Weatherhead, P.J., and S.B. McRae, 1990, "Brood Care in American Robins: Implications for Mixed Reproductive Strategies by Females," Animal Behavior 39:1179-1188.

Weber, C.W., et al., 1968, “Strontium Metabolism in Chicks,” Poultry Science 47:1318-1328.

Whicker, F.W., and V. Schultz, 1982a, Radioecology: Nuclear Energy and the Environment, Vol. 1, CRC Press, Boca Raton, Fla.

Whicker, F.W., and V. Schultz, 1982b, Radioecology: Nuclear Energy and the Environment, Vol. 2, CRC Press, Boca Raton, Fla. 